\title{
Circulating monocytes in atherosclerosis : local or systemic actors?
}

Citation for published version (APA):

Otten, J. J. T. (2013). Circulating monocytes in atherosclerosis : local or systemic actors? [Doctoral Thesis, Maastricht University]. Uitgeverij BOXPress. https://doi.org/10.26481/dis.20131106jo

Document status and date:

Published: 01/01/2013

DOI:

10.26481/dis.20131106jo

Document Version:

Publisher's PDF, also known as Version of record

\section{Please check the document version of this publication:}

- A submitted manuscript is the version of the article upon submission and before peer-review. There can be important differences between the submitted version and the official published version of record.

People interested in the research are advised to contact the author for the final version of the publication, or visit the DOI to the publisher's website.

- The final author version and the galley proof are versions of the publication after peer review.

- The final published version features the final layout of the paper including the volume, issue and page numbers.

Link to publication

\footnotetext{
General rights rights.

- You may freely distribute the URL identifying the publication in the public portal. please follow below link for the End User Agreement:

www.umlib.nl/taverne-license

Take down policy

If you believe that this document breaches copyright please contact us at:

repository@maastrichtuniversity.nl

providing details and we will investigate your claim.
}

Copyright and moral rights for the publications made accessible in the public portal are retained by the authors and/or other copyright owners and it is a condition of accessing publications that users recognise and abide by the legal requirements associated with these

- Users may download and print one copy of any publication from the public portal for the purpose of private study or research.

- You may not further distribute the material or use it for any profit-making activity or commercial gain

If the publication is distributed under the terms of Article $25 \mathrm{fa}$ of the Dutch Copyright Act, indicated by the "Taverne" license above, 
Circulating Monocytes in Atherosclerosis:

Local or Systemic Actors? 
Layout: Jeroen Otten

Cover Design: Jeroen Otten

Printed by: Proefschriftmaken.nl | | Uitgeverij BOXPress

Published by: Uitgeverij BOXPress, Oisterwijk

ISBN: $\quad$ 978-90-8891-698-4

C2013 Jeroen Otten, Munstergeleen 


\title{
Circulating Monocytes in Atherosclerosis: Local or Systemic Actors?
}

\author{
PROEFSCHRIFT \\ ter verkrijging van de graad van doctor aan de Universiteit Maastricht, \\ op gezag van de Rector Magnificus, Prof. Dr. L.L.G. Soete, \\ volgens het besluit van het College van Decanen, \\ in het openbaar te verdedigen
}

op woensdag 6 november 2013 om 12:00 uur

door

Jeroen Johannes Theresia Otten

geboren 5 november 1983 te Sittard 


\section{Promotor}

Prof. Dr. E.A.L. Biessen

\section{Beoordelingscommissie}

Prof. Dr. W.A. Buurman (Voorzitter)

Prof. Dr. M. de Baets

Prof. Dr. H. ten Cate

Dr. M. van Eck (Universiteit Leiden)

Dr. M.A. Nolte (Sanquin Research, Amsterdam)

This research was performed within the framework of CTMM, the Center for Translational Molecular Medicine (www.ctmm.nl), project CIRCULATING CELLS (grant 01C-102), and supported by the Netherlands Heart Foundation

Financial support by the Dutch Heart Foundation for the publication of this thesis is gratefully acknowledged 
Aan Stefanie and Finn, Voor al hun liefde en ondersteuning 



\section{Contents}

Chapter 01 General Introduction \& Thesis Outline 9

Chapter 02 Local but not systemic CD115 ${ }^{+}$myelocyte 35 ablation aggravates atherogenesis

Chapter 03 Hematopoietic G-protein-coupled receptor 65 kinase 2 deficiency decreases atherosclerotic lesion formation in LDL receptor- knockout mice

Chapter 04 Hypercholesterolemia exposed circulating 95 monocytes ingest lipids and induce proinflammatory pathways

Chapter 05 Phage display identifies novel circulating and plaque monocyte markers for advanced atherosclerosis

Chapter 06 Circulating unstable angina pectoris patient monocytes harbor no basal transcriptomic differences

Chapter 07 General Discussion \& Future Perspectives

Summary

Vereenvoudigde Nederlandse Samenvatting

Curriculum Vitae

List of Abbreviations

Dankwoord 


Cardiovascular diseases (CVD) are one of the leading causes of mortality and morbidity in the Western world. According to figures from the Netherlands Heart Foundation (NHF), approximately 40,000 people died from cardiac or vascular diseases in The Netherlands in $2010^{1}$. Although, the advances in research have already contributed to a decreased number of cardiovascular deaths in developed countries, still millions of people suffer from these diseases worldwide. Next to the successful intervention in developed countries, the incidence of CVD is believed to increase in developing countries over the next years, but also in the USA the prevalence of CVD is expected to increase ${ }^{2}$. Despite extensive research and the implementation of novel interventions not all patients at risk of a cardiovascular event can be detected timely or treated successfully. The knowledge on the consequences of risk factors, including obesity ${ }^{3}$, hypertension ${ }^{4}$, diabetes ${ }^{5}$, smoking ${ }^{6}$, and hyperlipidemia ${ }^{7}$, but also on disease detection and prediction need to be expanded even further, to obtain be able to truly develop personalized medicine strategies and resolve cardiovascular diseases on a global scale.

\section{Atherosclerosis}

Atherosclerosis is the key underlying cause of CVD, responsible for a significant number of cardiac and vascular events, such as stroke, myocardial infarction, and peripheral artery disease. Atherosclerosis is considered to be a chronic inflammatory disease of the medium and large arteries ${ }^{8}$. The disease is characterized by two main hallmarks: (I) lipid/lipoprotein metabolism and (II) inflammation. It is the interplay between these two processes that defines the principle pathogenesis and distinguishes atherosclerosis from other chronic inflammatory diseases ${ }^{9}$. Lipid and cholesterol accumulation in the subendothelial layer occurs at sites with disturbed flow, such as curvatures and bifurcations of the arteries ${ }^{10,11}$. The endothelial layer will hence become activated which is even further stimulated by accumulation of low density lipoprotein (LDL) cholesterol particles and the subsequent oxidation of these LDL particles ${ }^{12}$. This activated endothelium will produce inflammatory cytokines, but also increase the expression of adhesion receptors such as vascular cell adhesion molecule (VCAM) 1 and intercellular cell adhesion molecule (ICAM) 1 on the luminal membranes ${ }^{13-15}$. The upregulation of adhesion molecules will result in an increased attraction and adhesion of leukocytes to this activated endothelium ${ }^{16}$. Interestingly, the locations of the 
vasculature which have higher incidence of plaque development, already have increased leukocyte counts and signs of low-grade inflammation before development of atherosclerosis ${ }^{17}$ (figure 1 ).

Initially, the main leukocyte population attracted to the early atherosclerotic lesion will be monocytes. Monocytes will roll along the vessel wall and adhere at sites with increased adhesion receptors (or integrins). Eventually, the monocytes will attach to the endothelial layer of the vessel and can transmigrate into the subendothelial layer ${ }^{16,18}$. The inflammatory milieu of the intima will trigger the monocytes to differentiate into macrophages. The latter cell type is considered to be a tissue-resident, mature form of monocytes. Oxidized cholesterol present in the early lesion will be scavenged by these macrophages via class B scavenger receptors and CD36. However, as these cells are unable to metabolize these oxLDL molecules efficiently and cholesterol efflux is also inhibited, intra-cellular cholesterol will accumulate, giving rise to the formation of macrophage foam cells. These cells have a foamy appearance due to the presence of large amounts of cholesterol filled endosomes and cytoplasmic lipids ${ }^{19-22}$. The macrophages and foam cells present in the initial atherosclerotic lesions, together with other attracted leukocytes, such as T-lymphocytes, will produce pro-inflammatory cytokines and thereby stimulate further expansion of the lesion by attraction of additional leukocytes to the progressing atherosclerotic plaque.

Over time, advanced atherosclerotic lesions can develop, most often asymptomatic, and thus without any clinical symptoms for many years ${ }^{23,24}$. However, some lesions can progress into more unstable lesions by thinning of the fibrous cap covering the a-cellular and necrotic material present in the core of the lesion. Thinning of the cap is caused by e.g. matrix metallo-proteases (MMP), secreted by macrophages/foam cells in the lesion. Matrix metalloproteases have also been shown to be involved in proteolytic cleavage of some cytokines thereby stimulating the inflammatory response ${ }^{9}$. Eventual rupture of the cap will present the debris from the core of the lesion to the blood stream and trigger coagulation, giving rise to the formation of thrombi. These thrombi will in the end cause local or peripheral clinical symptoms and even death due to the occlusion of arteries and subsequent inhibition of blood flow 24. 


\section{Lipid/lipoprotein Metabolism in Atherosclerosis}

\section{Cholesterol synthesis and transportation}

Lipids and cholesterols are essential to most organisms as many processes depend on these compounds for normal function. Cholesterol is one of the main components of cell membranes and functions as precursor for steroid hormones ${ }^{25}$ and vitamin $\mathrm{D}^{26}$, while lipids in general are an important energy source for the body. As lipids and cholesterol are poorly soluble in aqueous solutions, they need to be encapsulated in order to be transported in the blood. Several different subsets of lipoproteins can be distinguished involved in cholesterol transport. The most important lipoprotein fractions are very low density lipoprotein (VLDL), low density lipoprotein (LDL), and high density lipoprotein (HDL). Generally, LDL is thought to be the 'bad' cholesterol ${ }^{27,28}$ and HDL the 'good' cholesterol ${ }^{29,30}$.

The liver plays a central role in the turnover of lipids and is responsible for the conversion of free fatty acids and chylomicrons into ApoB100-bound VLDL particles which are secreted into the circulation. Upon lipolysis, governed by lipoprotein lipases, VLDL is converted into LDL which is subsequently sequestered by the liver, but can also be used as metabolites in other pathways throughout the body. HDL particles are bound to ApoA-I which is important in the sequestration of cholesterol from cells via dedicated receptors . ATP-binding cassette transporter (ABCA) 1 is one of these receptors and is present on macrophages, supporting (limited) efflux of cholesterol to HDL particles ${ }^{31,32}$.

\section{Lipoproteins in Atherosclerosis}

Hypercholesterolemia is, already for many years now, considered one of the most important risk factors for the development of atherosclerosis ${ }^{33}$. Disturbances in the lipid/lipoprotein metabolism can affect the levels of various lipoprotein subsets, as these processes are responsible for the transport of lipoproteins throughout the circulation ${ }^{34}$. At sites with turbulent flow and thus decreased shear stress, LDL cholesterol can accumulate in the intima, where it is prone to oxidation by local radicals, resulting in the development of oxLDL. As mentioned, this will initiate atherogenesis by endothelial cell activation due to disturbed blood flow ${ }^{35,36}$. The humoral response to the oxLDL accumulation in the intima will further aggravate this 
immunological initiation of atherosclerosis ${ }^{37}$. The presence of lipoprotein, and more in particular oxLDL, is one of the central hallmarks of atherosclerosis. Exposure of (free) lipids and cholesterol, as well as necrotic material entrapped in the lesion to the blood flow upon plaque rupture may give rise to the clinical manifestations of cardiovascular disease ${ }^{24}$.

Next to free cholesterol, also cellular cholesterol contributes to atherogenesis. Oxidized LDL phagocytized by macrophages, stimulates the inflammatory response in the vessel wall by the release of pro-inflammatory cytokines from macrophage foam cells and the induction of necrosis of these cells. Intracellular cholesterol can be present either cytoplasmic or lysosomal. Further, cholesterol crystals can be observed in cells which have accumulated excessive amounts of lipids and cholesterol.

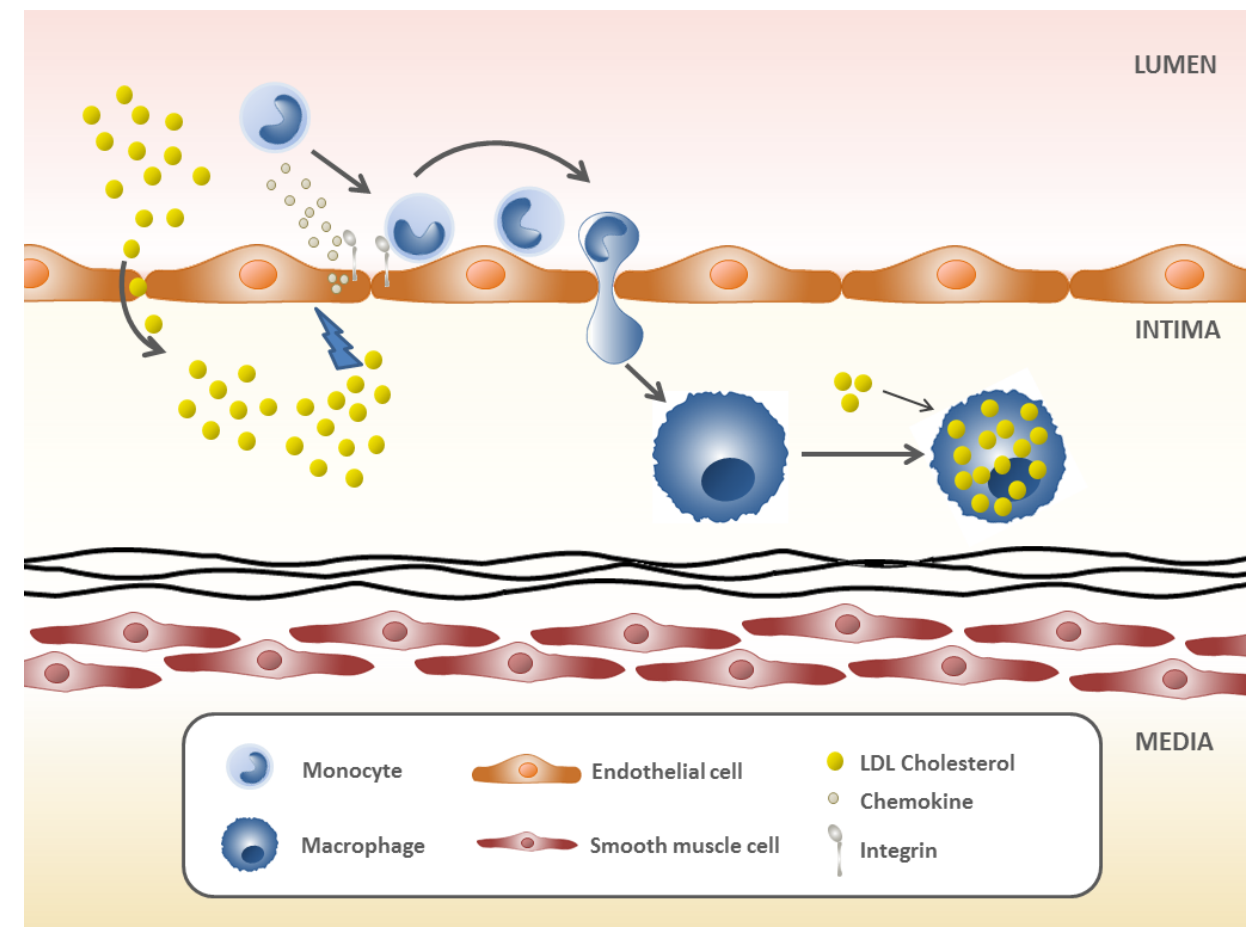

Figure 1 - Schematic overview of early atherosclerosis development

Atherosclerotic plaque development is initiated by low density lipoprotein (LDL) cholesterol accumulation in the subendothelial layer of the vessel wall. Free radicals present in the tissue will lead to the formation of oxidized LDL (oxLDL), resulting in activation of endothelial cells. Upon activation endothelial cells will release chemo-attractant cytokines (chemokines) and upregulate the expression of integrins, facilitating the adhesion, rolling, and transmigration of circulating monocytes. In the subendothelial tissue monocytes will differentiate into macrophages which will subsequently scavenge the cholesterol and stimulate the proinflammatory immune response. 


\section{Inflammation in Atherosclerosis}

Inflammation is, next to the lipid metabolism, a second essential process in the pathogenesis of atherosclerosis ${ }^{9}$. The inflammatory response consists of a cellular and humoral component and includes many immune cells, but also interactions with local endothelial cells and smooth muscle cells. As described earlier, circulating monocytes and plaque macrophages are critical actors in initial lesion development, but also in lesion progression, and eventual destabilization of the atherosclerotic plaque ${ }^{19}$.

\section{Monocyte subsets in human}

Human monocytes can be divided in two subsets based on expression of the membrane markers CD14 and CD16 ${ }^{38}$. In humans, CD14 is a co-receptor for binding of lipopolysaccharide (LPS) in combination with toll-like receptor (TLR) 4 and LPS-binding protein (LBP) ${ }^{39,40}$, while CD16 is a Fc receptor (FcrRIII) which bind the constant FC region of antibodies ${ }^{41}$. The two monocyte subsets that are currently being described in literature are $C D 14^{\text {high }} / \mathrm{CD} 16^{-}$and $\mathrm{CD} 14^{\text {low }} / \mathrm{CD} 16^{\text {t+ }}$. The most abundant monocyte population in the circulation is the 'classical' $\mathrm{CD} 14^{\text {high }} / \mathrm{CD} 16^{-}$monocytes, comprising over $90 \%$ of circulating monocytes ${ }^{42}$. The classical monocytes are also often defined as inflammatory monocytes ${ }^{43}$. The second population comprises the 'non-classical' $\mathrm{CD} 14^{\text {low }} / \mathrm{CD} 16^{\text {t+ }}$ monocytes, which are also referred to as patrolling monocytes. There are however also indications of a third monocyte population which has intermediate expression for both CD14 and CD16 (CD14 $\left.{ }^{+} / \mathrm{CD} 16^{+}\right)^{44}$.

The classical and non-classical monocyte populations show differential expression of chemokine receptors involved in chemotaxis towards inflammatory stimuli ${ }^{45}$. In relation to atherosclerosis and other inflammatory diseases such as rheumatoid arthritis, both subsets of circulating monocytes are thought to be important, however recruitment of these cells towards the site of inflammation is regulated by different chemokine receptors ${ }^{42,46}$. The CC-motif chemokine receptor 2 (CCR2) is expressed in high levels by 'classical' $\mathrm{CD} 14^{\text {high }} / \mathrm{CD} 16$ monocytes, and the responsiveness towards monocyte chemoattractant protein (MCP) 1 is considered to be a characteristic of this subset ${ }^{47}$. The $\mathrm{CX}_{3} \mathrm{C}$-motif chemokine receptor $1\left(\mathrm{CX}_{3} \mathrm{CR} 1\right)$, or fractalkine receptor, is expressed by both monocyte subsets ${ }^{48}$, however the expression is approximately two-fold higher in 'non-classical' monocytes ${ }^{49}$. This receptor is involved not only in chemotactic responses, but also has a role in cell survival 
${ }^{50,51}$. Other important chemokine receptors expressed by monocytes are CCR1 and CCR5 and to a lesser extent CXCR2, which also are important for the patrolling properties of monocytes together with CCR2 and $\mathrm{CX}_{3} \mathrm{CR} 1{ }^{47-49}$. As monocyte migration into the atherosclerotic lesion is mainly studied in mice there is only limited information on the role of these human subsets and their migratory behavior in atherosclerosis.

Table 1 - Monocyte subsets in human and mouse (Adapted from: Hristov et $a l^{52}$ )

Monocyte subset Alternative name Major markers

\begin{tabular}{lll}
\hline Human & & \\
CD14 $^{\text {high }}$ CD16 $^{-}$ & Classical/Inflammatory & CD14, CCR2, CD16, CD62L, SR-A, VEGFR1 \\
CD14 $^{\text {low }}$ CD16 $^{++}$ & Non-classical/patrolling & CD16, HLA-DR, CD11c, CX CR1, CCR5 \\
CD14 $^{+}$CD16 & Intermediate & CD14, CD16, CCR2, Tie2, CD105, MHCII, HLA-DR \\
$\begin{array}{l}\text { Mouse } \\
\text { Ly6C }^{\text {high }}\left(\text { Gr1 }^{+}\right)\end{array}$ & Classical & \\
Ly6C $^{-/ 10 w}\left(\right.$ Gr1 $\left.^{-}\right)$ & Patrolling & Ly6C/Gr1, CCR2, CD62L \\
\hline
\end{tabular}

\section{Monocyte subsets in mice}

In mice different circulating monocyte populations can be distinguished within the $\left(\mathrm{CD} 11 \mathrm{~b}^{+} / \mathrm{Ly} \mathrm{GG}^{-}\right)$monocytes population. In contrast to the human situation where monocyte subsets are defined based on CD14 and CD16 expression, in mice the subsets are identified based on Ly6C (or Gr1; binds both Ly6C and Ly6G) expression in combination with the general marker for myeloid cells (CD11b) and exclusion of granulocyte (Ly6G). Classical or inflammatory monocytes express high levels of Ly6C (or Gr1; Ly6C high monocytes), while patrolling or 'non-classical' monocytes express low levels of Ly6C (Ly6C $\mathrm{C}^{\text {low }}$ monocytes) ${ }^{48,53,54}$. As in human monocytes, the classical (Ly6C ${ }^{\text {high }}$ ) murine monocytes express high levels of CCR2, while patrolling (Ly6C ${ }^{\text {low }}$ ) monocytes do not express CCR2, but do express $\mathrm{CX}_{3} \mathrm{CR} 1$ and $\mathrm{CCR} 5{ }^{42,55}$.

Studies in mice have shown that the different monocyte populations reflect different stages of development ${ }^{56-58}$. The bone marrow produces Ly6 $\mathrm{C}^{\text {high }}$ monocytes, which can differentiate into Ly6 $6 \mathrm{C}^{\text {low }}$ monocytes in the periphery in the absence of inflammatory stimuli 55,59 . However, this progressive differentiation from Ly $6 C^{\text {high }}$ in the bone marrow to Ly $6 C^{\text {low }}$ in the periphery is still debated in literature ${ }^{60,61}$. Despite the similarities in chemokine receptor patterns for CCR2 and $\mathrm{CX}_{3} \mathrm{CR} 1$ (table 1), it is still questionable to what extent monocyte subsets in mouse and man can be compared directly, as in 
C57BI/6 mice both subpopulations are roughly present in equal numbers ${ }^{62}$. Nevertheless, it has been shown that circulating monocytes are essential for atherogenesis, as inhibition or depletion of (subsets of) monocytes results in reduced plaque development ${ }^{57,58}$ (figure 2 ).

\section{Monocyte/Macrophage Axis in Atherosclerosis}

Monocytes are the first leukocytes to enter the sub-endothelial space in response to lipoprotein accumulation in the early stages of atherogenesis. However, also during plaque progression and eventual plaque destabilization resulting in clinical manifestation of the disease, monocytes (and macrophages) are pivotal ${ }^{63,64}$. It has been shown that monocytes respond to tissue-derived signals by migrating into lymphoid and non-lymphoid tissues 65,66. Triggers for these tissue-derived signals can be tissue damage or infection. In atherosclerosis, it is however more complex as not only inflammatory signals, caused by modified cholesterol in the intima, but also disturbed laminar flow were seen to contribute to the recruitment of monocytes ${ }^{22,42}$. This also explains why atherosclerotic lesions are almost exclusively detected in curvatures and bifurcations of arteries ${ }^{11}$. Adhesion molecules on endothelial cells facilitate the adhesion of circulating monocytes to the arterial wall, and subsequently aid in the transmigration into the intima ${ }^{13-15}$. This process is supported by chemokines secreted by activated endothelial cells and inflammatory cells in the lesion.

For human disease the chronology of leukocyte plaque invasion is not completely understood, as tracking and fate-mapping studies are almost impossible in the human situation. In mice however, many studies have been performed in which monocytes have been tracked or depleted to get more insight in the processes involved in atherosclerosis development and the role of monocytes herein. Ly $6 C^{\text {high }}$ monocytes have been show to expand under hypercholesterolemic conditions and promote the inflammatory processes during atherogenesis ${ }^{67}$. The role of $\mathrm{Ly} 6 \mathrm{C}^{\text {low }}$ monocytes is more debatable in the development of atherosclerosis, as these monocytes are often considered to be anti-inflammatory and are traditionally thought to give rise to antiinflammatory $\mathrm{M} 2$ macrophages and dendritic cells. This dogma is being challenged nowadays with our growing understanding of monocyte development. 


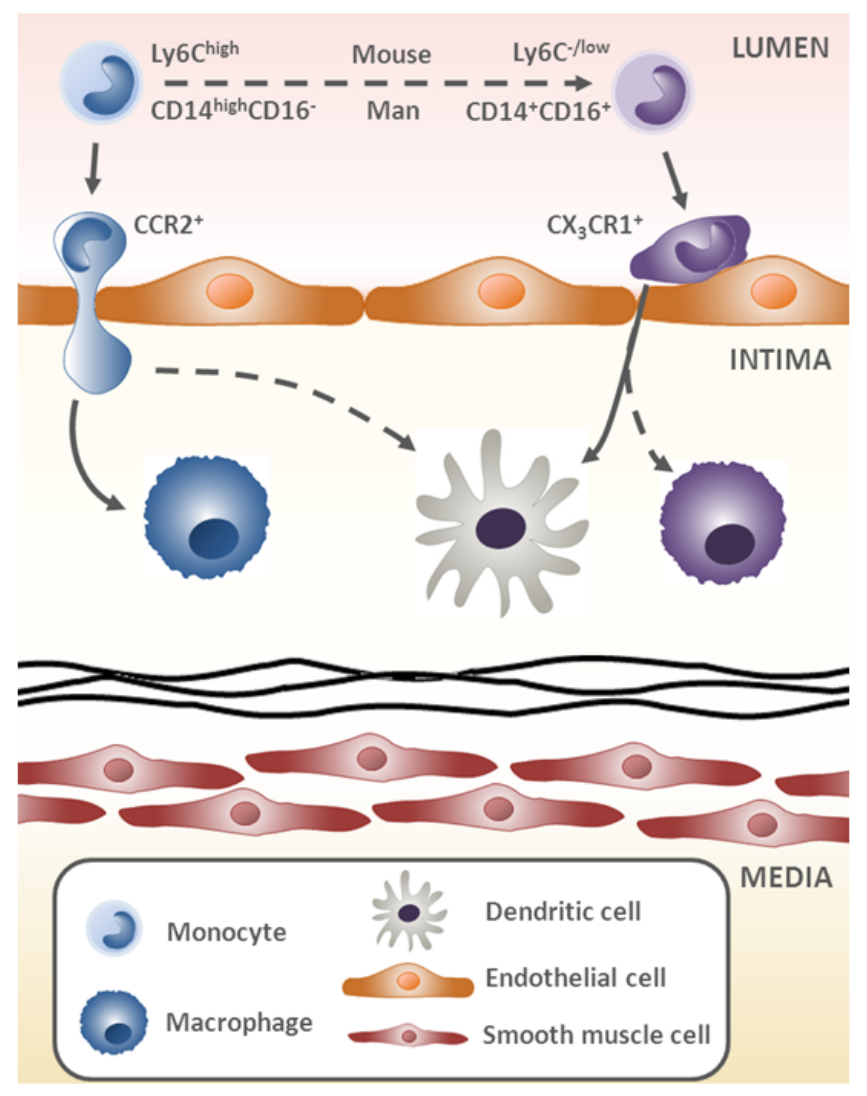

Figure 2 - Monocyte subsets in atherogenesis

Two important monocyte populations are described in mouse and humans related to atherosclerosis. On the one hand, the inflammatory Ly6 $\mathrm{C}^{\text {high }}$ (mouse) or CD14 ${ }^{\text {high }} / \mathrm{CD} 16^{-}$(human) monocytes which mainly migrate towards inflammatory sides such as the atherosclerotic lesion via CCR2 and predominantly give rise to (tissue-resident) macrophages. On the other hand, the patrolling $\mathrm{Ly}_{6 \mathrm{C}}$-/low (mouse) or $\mathrm{CD}^{+} 4^{+} / \mathrm{CD} 16^{+}$(human) monocytes which mainly migrate via $\mathrm{CX}_{3} \mathrm{CR} 1$ and give rise to both myeloid dendritic cells and macrophages.

\section{Macrophages in Atherosclerosis}

Macrophages are more comparable between mouse and man, as the subsets present in man are also observed in mice. Monocytes that infiltrate the tissue or inflammatory site (e.g. atherosclerotic lesion) differentiate into M0 macrophages. These macrophages are considered to be non-polarized and do not have specific pro- or anti-inflammatory properties. Polarization of these macrophages towards a pro- or anti-inflammatory phenotype is dependent on the local milieu ${ }^{68-70}$. 
Macrophages, as part of the innate immune system, are responsible for the scavenging of foreign materials. In the atherosclerotic lesion these cells will ingest the oxidized LDL from the intima. However, this process results in cell activation and thereby propagating the already ongoing immune response. Cytokines and proteases secreted by macrophages have an important role in plaque development and destabilization of the fibrous cap covering the atheroma $^{\text {9,71,72. }}$.

Local stimuli such as cytokines and cell-cell interactions can stimulate these tissue-resident macrophages to polarize into either classically activated, pro-inflammatory (M1) macrophages or alternatively activated, antiinflammatory (M2) macrophages ${ }^{68,69}$. Classically activated macrophages are considered to be the immune effector cells which are involved in the engulfment and digestion of microbes and other foreign particles. These cells are also important mediators of the inflammatory response and next to this have high proteolytic capacity. M1 macrophages are activated by lipopolysaccharide (LPS) and interferon (IFN) $\curlyvee$ and produce high levels of interleukin (IL) 12, while no or low levels of IL10 are produced. Other cytokines produced by M1 macrophages are IL1 $\beta$, IL6, and tumor necrosis factor (TNF) $\alpha$ ${ }^{70}$. Alternatively activated macrophages (M2) macrophages can be divided in $\mathrm{M} 2 \mathrm{a}$ and $\mathrm{M} 2 \mathrm{c}$ macrophages based on their membrane marker expression and cytokine production. In contrast to 'inflammatory' M1 macrophages, M2 macrophages are considered to be anti-inflammatory or wound-healing cells ${ }^{70,73}$. Polarization towards $\mathrm{M} 2 \mathrm{a}$ or $\mathrm{M} 2 \mathrm{c}$ phenotype can be induced by stimulation with IL4, or with IL10 and transforming growth factor (TGF) $\beta$, respectively ${ }^{73}$. These $M 2$ macrophages mainly produce IL10, stimulating antiinflammatory pathways in e.g. T-lymphocytes ${ }^{9,70,73}$.

In atherosclerosis the role of the different macrophage subsets has not yet been elucidated completely. Nevertheless, studies indicate that a heterogeneous set of macrophage subsets is present in the lesion ${ }^{68,69}$. Besides the well-known pro-inflammatory $\mathrm{M} 1$, and anti-inflammatory $\mathrm{M} 2 \mathrm{a}$ and $\mathrm{M} 2 \mathrm{c}$ macrophages, also oxLDL polarized macrophages (Mox) ${ }^{74}$ and CXCL4 polarized macrophages (M4) ${ }^{75}$ can be found in atherosclerotic plaques ${ }^{70,76}$. Due to the complexity of determining distinct macrophage subsets in the lesion in combination with potential phenotype switching of lesional macrophages ${ }^{77,78}$ the exact role of the different subsets in atherosclerosis development remains uncertain. 


\section{Hematopoietic Compartments}

The development of atherosclerotic lesions is considered to be a local pathology, however plaque rupture can result in systemic effects and even death due to e.g. myocardial infarction or stroke. As the cells present in the lesion are attracted from the periphery via the circulation and also cytokines and chemokines are released into the circulation, there is also an important systemic contribution. Recently, several studies have shown that there is indeed an important role for the bone marrow and the spleen, which are both involved in myelopoiesis, and thus in the production of monocytes ${ }^{59,79-81}$.

In mice it has been shown that atherosclerosis in combination with cardiovascular risk factors such as hyperlipidemia, result in systemic changes and adaptations of the hematopoietic process. Mainly, myeloid hematopoiesis (myelopoiesis) is affected, and leads to dynamic changes in bone marrow and spleen ${ }^{59,80}$. Increased numbers of monocytes (monocytosis) and also granulocytes can be detected upon cholesterol rich diet feeding in both mouse and man ${ }^{82,83}$. However, it is still disputable to what extent these changes are caused by hyperlipidemia, inflammation, or the combination of both factors. Despite the fact that still many questions need to be investigated, it is clear that hematopoiesis plays an important role in not only replenishing the pool of peripheral leukocytes and tissue-resident inflammatory cells, but also in boosting and regulating various inflammatory diseases. 


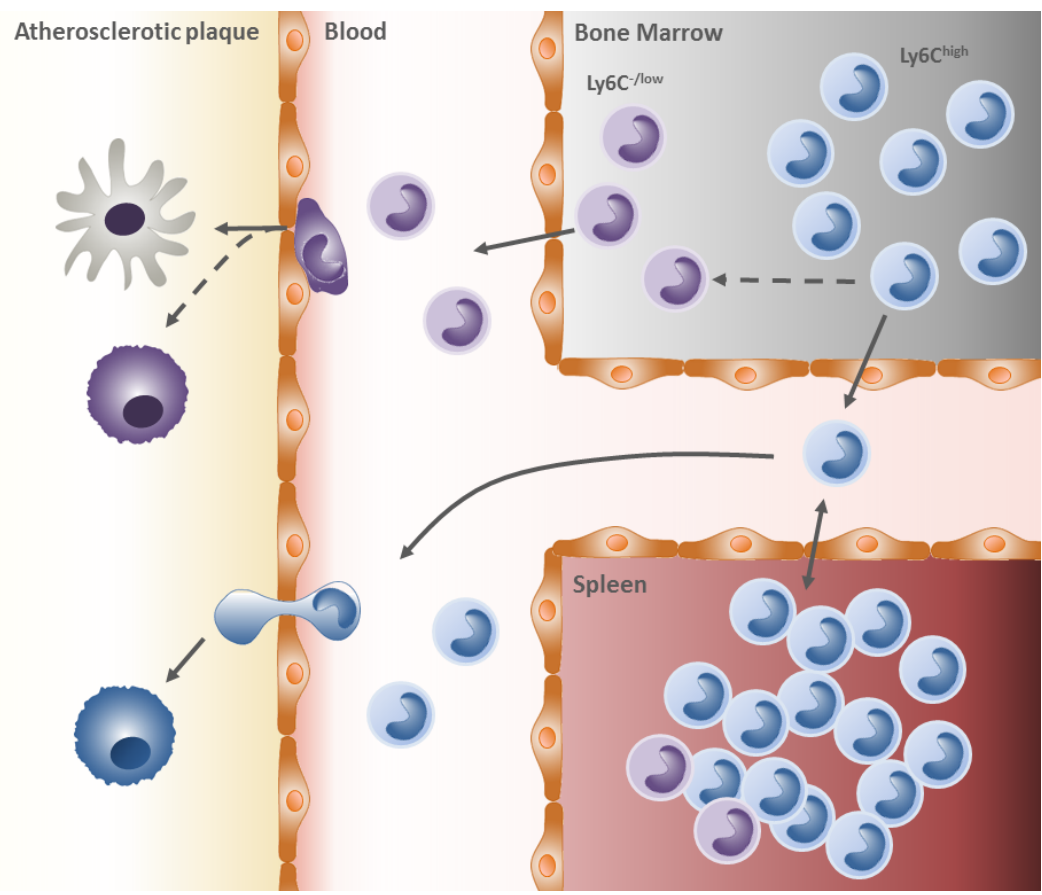

Figure 3 - Myelopoiesis in pro-atherogenic mouse models

Inflammatory stimuli in combination with hypercholesterolemia trigger the release of myeloid progenitor cells as well as mature monocytes from the bone marrow into the circulation. Myeloid progenitor cells will preferentially migrate towards the spleen, giving rise to extramedullar hematopoiesis. In combination with accumulation of bone marrow-derived monocytes in the spleen, a splenic reservoir will be formed consisting of (mainly Ly6 $6 \mathrm{C}^{\text {high }}$ ) monocytes. Upon additional stimulus, these cells are quickly mobilized from the spleen and migrate towards e.g. atherosclerotic lesions to form plaque macrophages and dendritic cells.

In normal myelopoiesis we can distinguish several intermediate myeloid precursor subsets developing from multipotent hematopoietic stem cells in the bone marrow. Common myeloid progenitors (CMP) develop via granulocyte/macrophage progenitors (GMP) and eventually mature granulocytes and monocytes. This development is controlled by cytokines, such as granulocyte/macrophage colony stimulating factor (GM-CSF), granulocyte colony stimulating factor (G-CSF), and macrophage colony stimulating factor (M-CSF) ${ }^{84-87}$. During development of monocytes, and their differentiation into macrophages, different transcription factors are expressed. Initially, PU.1 and Kruppel-like factor (KIf) 4 are activated, while later in the differentiation process Early growth response protein (Egr) 1 and interferon 
regulating factor (IRF) 8 are more important ${ }^{88,89}$. The expression of these transcription factors is specific to cells of the myeloid lineage and more specifically monocytes and macrophages ${ }^{79}$. IRF8 deficiency can also influence atherosclerosis development in mouse models indicating the importance of regulating and maintaining normal myelopoiesis ${ }^{90}$.

Besides the bone marrow, the spleen is also an important compartment in atherosclerosis-associated myelopoiesis. According to studies performed in pro-atherogenic apolipoprotein $\mathrm{E}$ deficient $\left(A p o E^{-/}\right)$mice, Ly6 $\mathrm{C}^{\text {high }}$ monocytes migrate from the bone marrow into the circulation ${ }^{59,80,83}$. Subsequently, these cells will migrate into the spleen and differentiate towards Ly6C $^{\text {low }}$ monocytes ${ }^{91}$. The spleen thus serves as a reservoir for rapid mobilization of monocytes upon inflammatory stimuli. In a pro-inflammatory system, such as observed in atherosclerosis, not only mature cells are released from the bone marrow, but also progenitors will be mobilized. These hematopoietic progenitors will home to the spleen and locally start producing mature myeloid cells. This extra-medullar hematopoiesis is one of the mechanisms responsible for the monocytosis observed in hypercholesterolemic, but also hyperglycemic, pro-atherogenic mice ${ }^{92,93}$. Overall, hematopoiesis contributes to atherosclerosis through enhanced production of myeloid cells and more specifically monocytes (figure 3 ).

\section{From Cells to Biomarkers}

Early detection and the selection of the optimal procedure for treatment are important aspects of cardiovascular biomarker research. Traditionally, different types of biomarkers can be distinguished and can be divided in diagnostic, prognostic, and predictive biomarkers. Diagnostic biomarkers are used to identify the presence of disease in a patient. These markers can be used in a clinical setting e.g. to distinguish between chest pain caused by myocardial infarction and chest pain due to non-cardiac related causes. Prognostic and predictive biomarkers give information on future risk of developing a specific event or disease. Prognostic biomarkers give information in untreated individuals, while predictive biomarkers can give more insight in the effectiveness of a treatment for individual patients. Both prognostic and predictive biomarkers can give indications for adapting the treatment.

Risk prediction for cardiovascular disease can be based on circulating, imaging, and genetic biomarkers ${ }^{94}$. Traditionally, risk prediction is mainly 
based on risk factors as hyperlipidemia, hypertension, diabetes mellitus, and smoking. These markers have been developed into predictive models such as the Framingham risk score ${ }^{95}$. Research has shown that these models for assessing CVD risk at population level are limited in predicting the risk for individual patients, due to changes in biomarkers upon treatment ${ }^{96}$ and lack of significant differences at the level of individual patients ${ }^{97,98}$.

In recent years, many novel biomarkers have been presented which are developed in new (high-throughput) platforms ${ }^{99}$. Based on the more traditional lipid and lipoprotein profiling additional biomarkers have been proposed. The ratio between apolipoprotein (Apo) B and ApoA-I, which are part of $L D L$ and $H D L$, respectively, is a strong predictive marker of cardiovascular events ${ }^{100,101}$. Another well-known biomarker for cardiovascular disease is C-reactive protein (CRP), which is a marker for systemic inflammation and is used in the clinic for many years ${ }^{102}$. The downside of CRP, and many more biomarkers in general, is the fact that different cohort studies result in different risk predictions ${ }^{103-105}$. Further, CRP is not specific for CVD as it is a more general marker for inflammation. Finally, biomarkers proposed so far have limited in their predictive value for an individual patient and often the biomarkers are inadequate with regard to specificity and sensitivity upon validation. Nevertheless, current and future biomarkers will contribute to personalized medicine extensively by improving diagnosis and prognosis for individual patients. By optimizing these strategies treatment and life style changes can be even more specified to the needs of a specific person, minimizing the risk for future cardiovascular morbidity and mortality. 
Table 2 - Non-comprehensive list of CVD biomarkers

\begin{tabular}{lllc} 
Biomarker & & Pathology & Ref \\
\hline CRP & C reactive protein & Acute inflammation & 102,104 \\
ApoB/ApoA-I & Apolipoprotein B/A-I & Predictive marker CV events & 100,101 \\
BNP & B-type natriuretic peptide & Prognostic marker heart failure & 106,107 \\
SCD40L & Soluble CD40 ligand & Plaque instability & 108 \\
CCL18 & CC-motif chemokine ligand 18 & Unstable angina pectoris & 109 \\
hsTNT & High sensitive troponin T & Myocardial injury & $107,110,111$ \\
SPLA 2 & Secreted phospholipases A2 & Prognostic marker CV events & 112 \\
MPO & Myeloperoxidase & Risk for future CV events & 113,114 \\
GDF15 & Growth differentiation factor 15 & Heart failure & 107,115 \\
\hline
\end{tabular}

Whole cells and also circulating monocytes are already being used as biomarker for disease. Both secreted and membrane-bound markers are related to different pathological conditions. Peripheral blood mononuclear cells (PBMCs) from patients with systemic sclerosis secrete more CD163, which might give information on prognosis ${ }^{116}$. In Alzheimer's disease LPS induced IL-6 release by PBMCs functions as prognostic marker for disease severity and cognitive outcome ${ }^{117}$. Besides these secreted proteins also membrane markers can be used as biomarker. Monocyte HLA-DR expression has a strong relationship with disease severity and prognosis in acute liver failure ${ }^{118}$, while Sialic acid-binding Ig-like lectin 1 (Siglec-1) and Fcrreceptor I (CD64) expression function as biomarker in systemic lupus erythromatosus ${ }^{119,120}$. For cardiovascular disease only one biomarker is described that is possibly derived from (plaque) monocytes, however the exact cell type responsible for the secretion of junction plakoglobin has not been determined yet ${ }^{121}$. As monocytes play a critical role in the development of atherosclerosis, in depth research towards biomarker discovery based on these cells will be necessary, to be able to more efficiently diagnose and treat patients at risk for CVD. 


\section{Focus \& Outline of the Thesis}

Despite the fact that in some developed countries the morbidity and mortality of cardiovascular diseases is diminishing, there is still need for more research as the incidence of disease is increasing in developing countries, and current diagnostic tools as well as therapies appear to be insufficient. Furthermore, there is a need for earlier, predictive and less invasive methods to detect the risk for atherosclerosis associated disorders. It is believed that circulating cells are the new target to determine the state of disease and risk for future events in patients suspected to have progressed cardiovascular diseases. This thesis focuses on the role of circulating monocytes in relation to atherosclerotic plaque development on the one hand, and the possibility of using these cells as biomarker source for plaque phenotype, and possibly even the risk for a future cardiovascular event on the other hand.

\section{Circulating monocytes in mouse models of atherosclerosis}

In Chapter 2, the effect of depleting monocytes and macrophages from the circulation, but also the atherosclerotic lesion will be investigated, using a proatherogenic mouse model. As the monocyte/macrophage axis plays a central role in atherosclerosis, depleting these subsets should have profound impact on local lesion associated processes, but also shed new light on the systemic importance of circulating cells in atherosclerosis.

Subsequently, the role of monocyte chemotaxis towards the atherosclerotic lesion will be analyzed in Chapter 3. We aim to gain insight into the processes regulating mobilization of monocytes from the periphery upon chemokine release. This chapter addresses the role GRK2 plays in chemotaxis and the effects of hematopoietic and macrophage specific deficiency on atherosclerotic lesion development in LDL receptor knock-out mice.

As atherosclerosis development is also critically dependent on lipids, and more in particular cholesterol, Chapter 4, will address the role of hypercholesterolemia on circulating monocytes. The processes underlying foam cell formation in the lesion are already well described, however little is known on the peripheral processes and responses of leukocytes, and in particular monocytes, upon increased cholesterol levels. Through diet-induced hypercholesterolemia, we are able to unravel the systemic adaptations, as well as functional changes in circulating monocytes. 


\section{Circulating monocytes as biomarker in human atherosclerotic disease}

The second part of this thesis focuses on the role of monocytes (and macrophages) in human cardiovascular disease.

First, in Chapter 5, the effects of cell activation, as observed in atherosclerosis, will be investigated on a protein level. Whole cell phage display is used to identify novel markers on human monocytes, which are specific for unstable angina pectoris and also advanced atherosclerotic lesions.

Subsequently, in Chapter 6, monocytes from stable and unstable angina pectoris patients will be studied on a mRNA level to determine whether there are baseline differences in monocyte activation or regulation due to the presence of unstable atherosclerotic plaques. Micro-array analysis, combined with computational analyses and flow cytometry are used to determine the profile of these monocyte pools, to verify whether monocytes from different patient groups do indeed have a different profile. We also include preliminary data on the differences in activation potential between monocytes from patients with an event during follow-up compared to controls.

Finally, a summary, general discussion of the results of this thesis, and future perspectives will be presented in Chapter 7 . 


\section{References}

1. Werkgroep Cijfers van de Hartstichting. Hart- en vaatziekten in Nederland 2011; Cijfers over leefstijl- en risicofactoren, ziekte en sterfte; 2011.

2. Sanz J, Moreno PR, Fuster V. The year in atherothrombosis. J Am Coll Cardiol. 2012;60(10):932-942.

3. Hubert HB, Feinleib M, McNamara PM, Castelli WP. Obesity as an independent risk factor for cardiovascular disease: a 26-year follow-up of participants in the Framingham Heart Study. Circulation. 1983;67(5):968-977.

4. Antikainen R, Jousilahti $P$, Tuomilehto J. Systolic blood pressure, isolated systolic hypertension and risk of coronary heart disease, strokes, cardiovascular disease and all-cause mortality in the middle-aged population. J Hypertens. 1998;16(5):577-583.

5. Kannel WB, McGee DL. Diabetes and cardiovascular disease. The Framingham study. JAMA. 1979;241(19):2035-2038.

6. Vander Zwaag R, Lemp GF, Hughes JP, et al. The effect of cigarette smoking on the pattern of coronary atherosclerosis. A case-control study. Chest. 1988;94(2):290295.

7. Stamler J, Daviglus ML, Garside DB, Dyer AR, Greenland P, Neaton JD. Relationship of baseline serum cholesterol levels in 3 large cohorts of younger men to long-term coronary, cardiovascular, and all-cause mortality and to longevity. JAMA. 2000;284(3):311-318.

8. Ross R. Atherosclerosis--an inflammatory disease. $N$ Engl J Med. 1999;340(2):115-126.

9. Halvorsen B, Otterdal K, Dahl TB, et al. Atherosclerotic plaque stability--what determines the fate of a plaque? Prog Cardiovasc Dis. 2008;51(3):183-194.

10. Zand T, Hoffman AH, Savilonis BJ, et al. Lipid deposition in rat aortas with intraluminal hemispherical plug stenosis. A morphological and biophysical study. Am J Pathol. 1999;155(1):85-92.

11. Nigro $P$, Abe J, Berk BC. Flow shear stress and atherosclerosis: a matter of site specificity. Antioxid Redox Signal. 2011;15(5):1405-1414.

12. Steinberg D. Low density lipoprotein oxidation and its pathobiological significance. J Biol Chem. 1997;272(34):20963-20966.

13. Ley K, Laudanna C, Cybulsky MI, Nourshargh S. Getting to the site of inflammation: the leukocyte adhesion cascade updated. Nat Rev Immunol. 2007;7(9):678-689.

14. Cybulsky MI, liyama K, Li H, et al. A major role for VCAM-1, but not ICAM-1, in early atherosclerosis. J Clin Invest. 2001;107(10):1255-1262.

15. Davies PF. Endothelial mechanisms of flow-mediated athero-protection and susceptibility. Circ Res. 2007;101(1):10-12.

16. Joris I, Zand T, Nunnari JJ, Krolikowski FJ, Majno G. Studies on the pathogenesis of atherosclerosis. I. Adhesion and emigration of mononuclear cells in the aorta of hypercholesterolemic rats. Am J Pathol. 1983;113(3):341-358.

17. Jongstra-Bilen J, Haidari M, Zhu SN, Chen M, Guha D, Cybulsky MI. Low-grade chronic inflammation in regions of the normal mouse arterial intima predisposed to atherosclerosis. J Exp Med. 2006;203(9):2073-2083. 
18. Luscinskas FW, Kansas GS, Ding $H$, et al. Monocyte rolling, arrest and spreading on IL-4-activated vascular endothelium under flow is mediated via sequential action of L-selectin, beta 1-integrins, and beta 2 -integrins. J Cell Biol. 1994;125(6):1417-1427.

19. Lusis AJ. Atherosclerosis. Nature. 2000;407(6801):233-241.

20. Suzuki $H$, Kurihara $Y$, Takeya $M$, et al. A role for macrophage scavenger receptors in atherosclerosis and susceptibility to infection. Nature. 1997;386(6622):292-296.

21. Febbraio M, Podrez EA, Smith JD, et al. Targeted disruption of the class B scavenger receptor $\mathrm{CD} 36$ protects against atherosclerotic lesion development in mice. J Clin Invest. 2000;105(8):1049-1056.

22. Westhorpe CL, Dufour EM, Maisa A, Jaworowski A, Crowe SM, Muller WA. Endothelial cell activation promotes foam cell formation by monocytes following transendothelial migration in an in vitro model. Exp Mol Pathol. 2012;93(2):220-226.

23. Burke AP, Farb A, Kolodgie FD, Narula J, Virmani R. Atherosclerotic plaque morphology and coronary thrombi. J Nucl Cardiol. 2002;9(1):95-103.

24. Virmani R, Kolodgie FD, Burke AP, Farb A, Schwartz SM. Lessons from sudden coronary death: a comprehensive morphological classification scheme for atherosclerotic lesions. Arterioscler Thromb Vasc Biol. 2000;20(5):1262-1275.

25. Hanukoglu I. Steroidogenic enzymes: structure, function, and role in regulation of steroid hormone biosynthesis. J Steroid Biochem Mol Biol. 1992;43(8):779-804.

26. Armbrecht HJ, Okuda K, Wongsurawat N, Nemani RK, Chen ML, Boltz MA. Characterization and regulation of the vitamin D hydroxylases. J Steroid Biochem Mol Biol. 1992;43(8):1073-1081.

27. Carmena R, Duriez P, Fruchart JC. Atherogenic lipoprotein particles in atherosclerosis. Circulation. 2004;109(23 Suppl 1):III2-7.

28. Samson S, Mundkur L, Kakkar VV. Immune response to lipoproteins in atherosclerosis. Cholesterol. 2012;2012:571846.

29. Haas MJ, Mooradian AD. Inflammation, high-density lipoprotein and cardiovascular dysfunction. Curr Opin Infect Dis. 2011;24(3):265-272.

30. Saemann MD, Poglitsch M, Kopecky C, Haidinger M, Horl WH, Weichhart T. The versatility of HDL: a crucial anti-inflammatory regulator. Eur J Clin Invest. 2010;40(12):1131-1143.

31. Rader DJ, Tall AR. The not-so-simple HDL story: Is it time to revise the HDL cholesterol hypothesis? Nat Med. 2012;18(9):1344-1346.

32. Hegele RA. Plasma lipoproteins: genetic influences and clinical implications. Nat Rev Genet. 2009;10(2):109-121.

33. Heber D, Koziol BJ, Henson LC. Low density lipoprotein receptor regulation and the cellular basis of atherosclerosis: implications for nutritional and pharmacologic treatment of hypercholesterolemia. Am J Cardiol. 1987;60(12):4G-8G.

34. Rader DJ, Daugherty A. Translating molecular discoveries into new therapies for atherosclerosis. Nature. 2008;451(7181):904-913.

35. World CJ, Garin G, Berk B. Vascular shear stress and activation of inflammatory genes. Curr Atheroscler Rep. 2006;8(3):240-244. 
36. Chiu JJ, Usami S, Chien S. Vascular endothelial responses to altered shear stress: pathologic implications for atherosclerosis. Ann Med. 2009;41(1):19-28.

37. Mestas J, Ley K. Monocyte-endothelial cell interactions in the development of atherosclerosis. Trends Cardiovasc Med. 2008;18(6):228-232.

38. Passlick B, Flieger D, Ziegler-Heitbrock HW. Identification and characterization of a novel monocyte subpopulation in human peripheral blood. Blood. 1989;74(7):2527-2534.

39. Kitchens RL. Role of CD14 in cellular recognition of bacterial lipopolysaccharides. Chem Immunol. 2000;74:61-82.

40. Wright SD, Ramos RA, Tobias PS, Ulevitch RJ, Mathison JC. CD14, a receptor for complexes of lipopolysaccharide (LPS) and LPS binding protein. Science. 1990;249(4975):1431-1433.

41. Clarkson SB, Ory PA. CD16. Developmentally regulated IgG Fc receptors on cultured human monocytes. J Exp Med. 1988;167(2):408-420.

42. Gautier EL, Jakubzick C, Randolph GJ. Regulation of the migration and survival of monocyte subsets by chemokine receptors and its relevance to atherosclerosis. Arterioscler Thromb Vasc Biol. 2009;29(10):1412-1418.

43. Ziegler-Heitbrock L, Ancuta P, Crowe S, et al. Nomenclature of monocytes and dendritic cells in blood. Blood. 2010;116(16):e74-80.

44. Zawada AM, Rogacev KS, Rotter B, et al. SuperSAGE evidence for CD14++CD16+ monocytes as a third monocyte subset. Blood. 2011;118(12):e50-61.

45. Wong KL, Tai JJ, Wong WC, et al. Gene expression profiling reveals the defining features of the classical, intermediate, and nonclassical human monocyte subsets. Blood. 2011;118(5):e16-31.

46. Katschke KJ, Jr., Rottman JB, Ruth JH, et al. Differential expression of chemokine receptors on peripheral blood, synovial fluid, and synovial tissue monocytes/macrophages in rheumatoid arthritis. Arthritis Rheum. 2001;44(5):10221032.

47. Zlotnik A, Yoshie O. Chemokines: a new classification system and their role in immunity. Immunity. 2000;12(2):121-127.

48. Woollard KJ, Geissmann F. Monocytes in atherosclerosis: subsets and functions. Nat Rev Cardiol. 2010;7(2):77-86.

49. Geissmann F, Jung S, Littman DR. Blood monocytes consist of two principal subsets with distinct migratory properties. Immunity. 2003;19(1):71-82.

50. Jakubzick C, Tacke F, Llodra J, van Rooijen N, Randolph GJ. Modulation of dendritic cell trafficking to and from the airways. J Immunol. 2006;176(6):3578-3584.

51. Landsman L, Bar-On L, Zernecke A, et al. CX3CR1 is required for monocyte homeostasis and atherogenesis by promoting cell survival. Blood. 2009;113(4):963972.

52. Hristov M, Weber C. Differential role of monocyte subsets in atherosclerosis. Thromb Haemost. 2011;106(5):757-762.

53. Tacke F, Alvarez D, Kaplan TJ, et al. Monocyte subsets differentially employ CCR2, CCR5, and CX3CR1 to accumulate within atherosclerotic plaques. J Clin Invest. 2007;117(1):185-194. 
54. Weber C, Zernecke A, Libby P. The multifaceted contributions of leukocyte subsets to atherosclerosis: lessons from mouse models. Nat Rev Immunol. 2008;8(10):802-815.

55. Sunderkotter C, Nikolic T, Dillon MJ, et al. Subpopulations of mouse blood monocytes differ in maturation stage and inflammatory response. J Immunol. 2004;172(7):4410-4417.

56. Auffray C, Fogg D, Garfa M, et al. Monitoring of blood vessels and tissues by a population of monocytes with patrolling behavior. Science. 2007;317(5838):666-670.

57. Saederup N, Chan L, Lira SA, Charo IF. Fractalkine deficiency markedly reduces macrophage accumulation and atherosclerotic lesion formation in CCR2-/- mice: evidence for independent chemokine functions in atherogenesis. Circulation. 2008;117(13):1642-1648.

58. Combadiere $C$, Potteaux S, Rodero $M$, et al. Combined inhibition of CCL2, CX3CR1, and CCR5 abrogates Ly6C(hi) and Ly6C(lo) monocytosis and almost abolishes atherosclerosis in hypercholesterolemic mice. Circulation. 2008;117(13):1649-1657.

59. Robbins CS, Chudnovskiy A, Rauch PJ, et al. Extramedullary hematopoiesis generates Ly-6C(high) monocytes that infiltrate atherosclerotic lesions. Circulation. 2012;125(2):364-374.

60. Geissmann F, Auffray C, Palframan R, et al. Blood monocytes: distinct subsets, how they relate to dendritic cells, and their possible roles in the regulation of T-cell responses. Immunol Cell Biol. 2008;86(5):398-408.

61. Varol C, Landsman L, Fogg DK, et al. Monocytes give rise to mucosal, but not splenic, conventional dendritic cells. J Exp Med. 2007;204(1):171-180.

62. Hilgendorf I, Swirski FK. Making a difference: monocyte heterogeneity in cardiovascular disease. Curr Atheroscler Rep. 2012;14(5):450-459.

63. Calin MV, Manduteanu I, Dragomir E, et al. Effect of depletion of monocytes/macrophages on early aortic valve lesion in experimental hyperlipidemia. Cell Tissue Res. 2009;336(2):237-248.

64. Yancey PG, Ding Y, Fan D, et al. Low-density lipoprotein receptor-related protein 1 prevents early atherosclerosis by limiting lesional apoptosis and inflammatory Ly-6Chigh monocytosis: evidence that the effects are not apolipoprotein E dependent. Circulation. 2011;124(4):454-464.

65. Volpe S, Cameroni E, Moepps B, Thelen S, Apuzzo T, Thelen M. CCR2 acts as scavenger for CCL2 during monocyte chemotaxis. PLoS One. 2012;7(5):e37208.

66. Leon B, Ardavin C. Monocyte migration to inflamed skin and lymph nodes is differentially controlled by L-selectin and PSGL-1. Blood. 2008;111(6):3126-3130.

67. Swirski FK, Weissleder R, Pittet MJ. Heterogeneous in vivo behavior of monocyte subsets in atherosclerosis. Arterioscler Thromb Vasc Biol. 2009;29(10):14241432.

68. Ley K, Miller YI, Hedrick CC. Monocyte and macrophage dynamics during atherogenesis. Arterioscler Thromb Vasc Biol. 2011;31(7):1506-1516.

69. Moore KJ, Tabas I. Macrophages in the pathogenesis of atherosclerosis. Cell. 2011;145(3):341-355.

70. Wolfs IM, Donners MM, de Winther MP. Differentiation factors and cytokines in the atherosclerotic plaque micro-environment as a trigger for macrophage polarisation. Thromb Haemost. 2011;106(5):763-771. 
71. Kampoli AM, Tousoulis D, Papageorgiou N, et al. Matrix metalloproteinases in acute coronary syndromes: current perspectives. Curr Top Med Chem. 2012;12(10):1192-1205.

72. Speidl WS, Kastl SP, Hutter R, et al. The complement component C5a is present in human coronary lesions in vivo and induces the expression of MMP-1 and MMP-9 in human macrophages in vitro. FASEB J. 2011;25(1):35-44.

73. Gordon S. Alternative activation of macrophages. Nat Rev Immunol. 2003;3(1):23-35.

74. Kadl A, Meher AK, Sharma PR, et al. Identification of a novel macrophage phenotype that develops in response to atherogenic phospholipids via Nrf2. Circ Res. 2010;107(6):737-746.

75. Gleissner CA, Shaked I, Little KM, Ley K. CXC chemokine ligand 4 induces a unique transcriptome in monocyte-derived macrophages. J Immunol. 2010;184(9):4810-4818.

76. Butcher MJ, Galkina EV. Phenotypic and functional heterogeneity of macrophages and dendritic cell subsets in the healthy and atherosclerosis-prone aorta. Front Physiol. 2012;3:44.

77. Khallou-Laschet J, Varthaman A, Fornasa G, et al. Macrophage plasticity in experimental atherosclerosis. PLoS One. 2010;5(1):e8852.

78. Feig JE, Rong JX, Shamir R, et al. HDL promotes rapid atherosclerosis regression in mice and alters inflammatory properties of plaque monocyte-derived cells. Proc Natl Acad Sci U S A. 2011;108(17):7166-7171.

79. Geissmann F, Manz MG, Jung S, Sieweke MH, Merad M, Ley K. Development of monocytes, macrophages, and dendritic cells. Science. 2010;327(5966):656-661.

80. Leuschner F, Rauch PJ, Ueno T, et al. Rapid monocyte kinetics in acute myocardial infarction are sustained by extramedullary monocytopoiesis. J Exp Med. 2012;209(1):123-137.

81. Ingersoll MA, Platt AM, Potteaux S, Randolph GJ. Monocyte trafficking in acute and chronic inflammation. Trends Immunol. 2011;32(10):470-477.

82. Tolani S, Pagler TA, Murphy AJ, et al. Hypercholesterolemia and reduced HDL$\mathrm{C}$ promote hematopoietic stem cell proliferation and monocytosis: Studies in mice and FH children. Atherosclerosis. 2013.

83. Swirski FK, Libby P, Aikawa E, et al. Ly-6Chi monocytes dominate hypercholesterolemia-associated monocytosis and give rise to macrophages in atheromata. J Clin Invest. 2007;117(1):195-205.

84. Liu B, Tan W, Barsoum A, et al. IL-17 is a potent synergistic factor with GM-CSF in mice in stimulating myelopoiesis, dendritic cell expansion, proliferation, and functional enhancement. Exp Hematol. 2010;38(10):877-884 e871.

85. Jack GD, Zhang L, Friedman AD. M-CSF elevates c-Fos and phosphoC/EBPalpha(S21) via ERK whereas G-CSF stimulates SHP2 phosphorylation in marrow progenitors to contribute to myeloid lineage specification. Blood. 2009;114(10):21722180.

86. Liongue C, Hall CJ, O'Connell BA, Crosier P, Ward AC. Zebrafish granulocyte colony-stimulating factor receptor signaling promotes myelopoiesis and myeloid cell migration. Blood. 2009;113(11):2535-2546. 
87. Barreda DR, Hanington PC, Belosevic M. Regulation of myeloid development and function by colony stimulating factors. Dev Comp Immunol. 2004;28(5):509-554.

88. Kurotaki D, Osato N, Nishiyama A, et al. Essential role of the IRF8-KLF4 transcription factor cascade in murine monocyte differentiation. Blood. 2013;121(10):1839-1849.

89. Yamamoto M, Kato T, Hotta $C$, et al. Shared and distinct functions of the transcription factors IRF4 and IRF8 in myeloid cell development. PLoS One. 2011;6(10):e25812.

90. Doring $\mathrm{Y}$, Soehnlein O, Drechsler M, et al. Hematopoietic interferon regulatory factor 8-deficiency accelerates atherosclerosis in mice. Arterioscler Thromb Vasc Biol. 2012;32(7):1613-1623.

91. Tacke F, Ginhoux F, Jakubzick C, van Rooijen N, Merad M, Randolph GJ. Immature monocytes acquire antigens from other cells in the bone marrow and present them to T cells after maturing in the periphery. J Exp Med. 2006;203(3):583597.

92. Nagareddy PR, Murphy AJ, Stirzaker RA, et al. Hyperglycemia promotes myelopoiesis and impairs the resolution of atherosclerosis. Cell Metab. 2013;17(5):695-708.

93. Soehnlein O, Swirski FK. Hypercholesterolemia links hematopoiesis with atherosclerosis. Trends Endocrinol Metab. 2013;24(3):129-136.

94. Ge Y, Wang TJ. Circulating, imaging, and genetic biomarkers in cardiovascular risk prediction. Trends Cardiovasc Med. 2011;21(4):105-112.

95. Wilson PW, D'Agostino RB, Levy D, Belanger AM, Silbershatz H, Kannel WB. Prediction of coronary heart disease using risk factor categories. Circulation. 1998;97(18):1837-1847.

96. Boyer B, Hart KW, Sperling MI, Lindsell CJ, Collins SP. Biomarker changes during acute heart failure treatment. Congest Heart Fail. 2012;18(2):91-97.

97. Goldberg RJ, Konstam MA. Assessing the population burden from heart failure: need for sentinel population-based surveillance systems. Arch Intern Med. 1999;159(1):15-17.

98. Shortreed SM, Peeters A, Forbes AB. Estimating the effect of long-term physical activity on cardiovascular disease and mortality: evidence from the Framingham Heart Study. Heart. 2013;99(9):649-654.

99. Wang TJ, Wollert KC, Larson MG, et al. Prognostic utility of novel biomarkers of cardiovascular stress: the Framingham Heart Study. Circulation. 2012;126(13):15961604.

100. Yusuf S, Hawken S, Ounpuu S, et al. Effect of potentially modifiable risk factors associated with myocardial infarction in 52 countries (the INTERHEART study): casecontrol study. Lancet. 2004;364(9438):937-952.

101. McQueen MJ, Hawken S, Wang X, et al. Lipids, lipoproteins, and apolipoproteins as risk markers of myocardial infarction in 52 countries (the INTERHEART study): a case-control study. Lancet. 2008;372(9634):224-233.

102. Kroop IG, Shackman NH. Level of C-reactive protein as a measure of acute myocardial infarction. Proc Soc Exp Biol Med. 1954;86(1):95-97. 
103. Alexander KS, Kazmierczak SC, Snyder CK, Oberdorf JA, Farrell DH. Prognostic utility of biochemical markers of cardiovascular risk: impact of biological variability. Clin Chem Lab Med. 2013:1-8.

104. Yousuf O, Mohanty BD, Martin SS, et al. High-sensitivity C-reactive Protein and Cardiovascular Disease: A Resolute Belief or an Elusive Link? J Am Coll Cardiol. 2013.

105. van Holten TC, Waanders LF, de Groot PG, et al. Circulating biomarkers for predicting cardiovascular disease risk; a systematic review and comprehensive overview of meta-analyses. PLoS One. 2013;8(4):e62080.

106. Bhalla V, Willis S, Maisel AS. B-type natriuretic peptide: the level and the drug-partners in the diagnosis of congestive heart failure. Congest Heart Fail. 2004;10(1 Suppl 1):3-27.

107. Santhanakrishnan R, Chong JP, Ng TP, et al. Growth differentiation factor 15, ST2, high-sensitivity troponin $\mathrm{T}$, and $\mathrm{N}$-terminal pro brain natriuretic peptide in heart failure with preserved vs. reduced ejection fraction. Eur J Heart Fail. 2012;14(12):13381347.

108. Wang JH, Zhang YW, Zhang $\mathrm{P}$, et al. CD40 ligand as a potential biomarker for atherosclerotic instability. Neurol Res. 2013.

109. Kraaijeveld AO, de Jager SC, de Jager WJ, et al. CC chemokine ligand-5 (CCL5/RANTES) and CC chemokine ligand-18 (CCL18/PARC) are specific markers of refractory unstable angina pectoris and are transiently raised during severe ischemic symptoms. Circulation. 2007;116(17):1931-1941.

110. Dinh W, Nickl W, Futh $\mathrm{R}$, et al. High sensitive troponin $\mathrm{T}$ and heart fatty acid binding protein: novel biomarker in heart failure with normal ejection fraction? $A$ cross-sectional study. BMC Cardiovasc Disord. 2011;11:41.

111. Freund $Y$, Chenevier-Gobeaux C, Bonnet $P$, et al. High-sensitivity versus conventional troponin in the emergency department for the diagnosis of acute myocardial infarction. Crit Care. 2011;15(3):R147.

112. Koenig W, Khuseyinova N. Lipoprotein-associated and secretory phospholipase A2 in cardiovascular disease: the epidemiological evidence. Cardiovasc Drugs Ther. 2009;23(1):85-92.

113. Wong ND, Gransar H, Narula J, et al. Myeloperoxidase, subclinical atherosclerosis, and cardiovascular disease events. JACC Cardiovasc Imaging. 2009;2(9):1093-1099.

114. Schindhelm RK, van der Zwan LP, Teerlink T, Scheffer PG. Myeloperoxidase: a useful biomarker for cardiovascular disease risk stratification? Clin Chem. 2009;55(8):1462-1470.

115. Wollert KC, Kempf T. Growth differentiation factor 15 in heart failure: an update. Curr Heart Fail Rep. 2012;9(4):337-345.

116. Bielecki M, Kowal K, Lapinska A, Chyczewski L, Kowal-Bielecka O. Increased release of soluble $\mathrm{CD} 163$ by the peripheral blood mononuclear cells is associated with worse prognosis in patients with systemic sclerosis. Adv Med Sci. 2013:13-20.

117. Kaplin A, Carroll KA, Cheng J, et al. IL- 6 release by LPS-stimulated peripheral blood mononuclear cells as a potential biomarker in Alzheimer's disease. Int Psychogeriatr. 2009;21(2):413-414. 
118. Antoniades CG, Berry PA, Davies ET, et al. Reduced monocyte HLA-DR expression: a novel biomarker of disease severity and outcome in acetaminopheninduced acute liver failure. Hepatology. 2006;44(1):34-43.

119. Li Y, Lee PY, Kellner ES, et al. Monocyte surface expression of Fcgamma receptor RI (CD64), a biomarker reflecting type-I interferon levels in systemic lupus erythematosus. Arthritis Res Ther. 2010;12(3):R90.

120. Biesen R, Demir C, Barkhudarova F, et al. Sialic acid-binding Ig-like lectin 1 expression in inflammatory and resident monocytes is a potential biomarker for monitoring disease activity and success of therapy in systemic lupus erythematosus. Arthritis Rheum. 2008;58(4):1136-1145.

121. Cooksley-Decasper S, Reiser H, Thommen DS, et al. Antibody phage display assisted identification of junction plakoglobin as a potential biomarker for atherosclerosis. PLoS One. 2012;7(10):e47985. 



\section{Abstract}

Although macrophage apoptosis is deemed a critical process in plaque progression, macrophage ablation studies have so far yielded contradictory results on the actual impact of macrophage death on atherosclerosis. This may be partly due to ectopic effects of the ablation strategy on circulating monocytes as well as on other leukocyte subsets. To distinguish plaque macrophage cell death associated effects from that on peripheral monocytic cells, we here studied the impact of systemic versus plaque-targeted ablation of monocytic cells on plaque development. ApoE ${ }^{-1-}$ mice, bearing a Macrophage FAS Induced Apoptosis (MaFIA) suicide gene under control of the CD115 promotor were used to develop advanced atherosclerotic lesions by perivascular collar placement in response to Western type diet feeding. Rapalog dimerizer was administered either systemically via intravenous tail vein injections, or locally via catheterized osmotic minipumps connected to the peri-adventitial site of the carotid arteries. Systemic treatment did not result in any changes regarding plaque development, despite efficient depletion of intimal macrophages. However, while effecting a complete ablation of circulating monocytes and neutrophils at 1 week after administration, systemic ablation led to a profoundly dysbalanced myelopoiesis, with major expansion of mature and immature myeloid cells in the circulation after 2 weeks. In contrast, focal ablation of CD115+ macrophages led to increased macrophage apoptosis and necrotic core size and to plaque expansion without any systemic effects. Taken together, extramedullar myelopoiesis and increased mobilization of inflammatory spleen monocytes to the circulation, upon systemic induction of monocyte/macrophage apoptosis causes vulnerability of atherosclerotic lesions. Local induction of macrophage apoptosis induced lesion expansion but allowed macrophage turnover without lesion vulnerability. 


\section{Introduction}

Macrophage accumulation in the atherosclerotic lesion is generally considered essential for plaque progression and destabilization ${ }^{1}$. Furthermore, macrophages exert a range of functions to control and clear infections ${ }^{2}$, regulate and modulate wound healing ${ }^{3}$, and engulf (non-)foreign particles ${ }^{4}$. In early atherosclerosis these myeloid cells are involved in the clearance of oxidized low density lipoproteins (oxLDL) from the vessel wall, whereas in later stages macrophages also mediate the clearance of apoptotic cell material from the lesion ${ }^{1,4}$. Hence, macrophage cell death will at later stages be associated with impaired efferocytosis, and deposition of macrophage contained lipid debris in the plaque, both processes deemed deleterious for plaque stability ${ }^{5}$. Moreover, macrophage derived matrix metallo-proteases (MMP) contribute to the degradation of the plaque-stabilizing collagen filaments and thereby undermine the integrity of the atherosclerotic lesion ${ }^{6,7}$.

Although plaque macrophage build up may partly result from clonal expansion of resident vascular macrophages as well, the bulk was seen to originate from extravasated monocytes that had been attracted to the plaque ${ }^{1,8}$. The recruitment of monocytes is a continuous process throughout all stages of the atherosclerotic lesion development ${ }^{9}$. Thus, by fostering macrophage accumulation, monocytes will contribute to the progression of lesions and more specifically to the perpetuation of the ongoing inflammatory state and the vulnerability of the advanced atherosclerotic lesion as well ${ }^{10,11}$. In support of this notion, human leukocytosis was demonstrated to be an independent risk factor and predictor of clinical cardiovascular events in atherosclerosis ${ }^{12-15}$.

This recognition has inspired to a wealth of studies on the interaction between of the myeloid lineage and hyperlipidemia and atherosclerosis. Our insights into the role of monocytes and macrophages in atherosclerosis have been greatly aided by both pharmacological and genetic monocyte/macrophage specific ablation studies. Clodronate liposomes have been used widely to study the effects of macrophage ablation in atherosclerosis ${ }^{16}$, but also other pathologies ${ }^{17,18}$. Calin et al reported that initially macrophage depletion in atherogenesis is beneficial, however increased lipid and collagen content ultimately results in plaque expansion. Furthermore, studies have applied diphtheria toxin dependent ablation models under control of $\mathrm{CD} 11 \mathrm{~b}^{19}$. Induction of macrophage apoptosis via a CD11b- 
diphteria toxin model showed that depletion of monocytes/macrophages is influencing early lesions more pronouncedly than established lesion ${ }^{19}$. CD11bhDTR and clodronate liposomes dependent myeloid cell depletion appear to indicate similar effects of monocyte/macrophage ablation on atherosclerosis development.

The key role of monocytes and macrophages in atherosclerosis development ${ }^{1}$, but also other inflammatory diseases such as rheumatoid arthritis ${ }^{20}$, has already been demonstrated. However, the aforementioned models for macrophage depletion are often lack specificity, and directly or indirectly impacted leukocyte subsets other than macrophages, thwarting data interpretation and resulting in unwanted side effects ${ }^{19,21-23}$. CD11b is expressed by not only monocytes/macrophages, but also by neutrophils and dendritic cells, in contrast, CD115 is more constricted to the monocyte/macrophage axis, and thus CD115 will give a more specific insight in the role of this axis in atherosclerosis development.

In this study we employed a loss-of-function approach using a MaFIA transgenic mouse model that carries an inducible FAS construct under control of the CD115 (also known as macrophage-colony stimulating factor receptor [M-CSFr]or colony stimulating factor 1 receptor [CSF-1R]) promotor ${ }^{24}$. In mouse and man, the monocytic lineage depends entirely on M-CSF for their ontogenesis ${ }^{25-27}$., and they produce and express high levels of this cytokine as well as of the cognate receptor CD115. Together with other cytokines such as granulocyte/macrophage colony stimulating factor (GM-CSF) and interferon gamma $(\mathrm{IFN}-\gamma)^{28}, \mathrm{M}-\mathrm{CSF}$ is essential for myeloid lineage commitment in the bone marrow ${ }^{29}$. The key role of the M-CSF receptor in the myelopoiesis leads to the concept of ablating macrophages based on this receptor to study the role of macrophages in atherosclerosis and myelopoiesis ${ }^{30}$. This model allows us to deplete $\mathrm{CD}_{115^{+}}$monocytes and macrophages in a time-controlled manner, by administering a dimerizer. Lymphocytes, and also granulocytes and dendritic cells, are considered to be M-CSF receptor low or negative and predominantly develop in an M-CSF independent manner ${ }^{31}$. 
We provide evidence that depletion of M-CSF receptor (CD115) positive myeloid cells in a FAS-dependent manner, only contributes to atherosclerotic lesion formation in mice on a pro-atherogenic Apo lipoprotein $\mathrm{E}$ deficient $\left(\mathrm{ApOE}^{-\top}\right)$ background when depleted locally in the atherosclerotic lesion. Systemic ablation of M-CSF receptor positive monocytes and macrophages does not influence atherosclerosis development, however it does result in deregulation of erythropoiesis and myelopoiesis.

\section{Materials \& Methods}

\section{Animals}

C57BL/6J-Tg(CSf1r-EGFP-NGFR/FKBP1A/TNFRSF6)2Bck/J MaFIA ${ }^{+/+}$male mice obtained from the Jackson Laboratories ${ }^{32}$, were backcrossed to $A p o E^{-/-}$mice for more than 12 generations to obtain homozygous $\mathrm{ApoE}^{/-} / / \mathrm{MaFIA}^{+/+}$mice. The MaFIA transgene was inherited according to Mendelian laws as demonstrated by qPCR analysis of gDNA (PureLink Genomic DNA Purification Kit, Invitrogen). Primer sequences were: 5'-CCACATGAAGCAGCAGGACTT-3' for the forward primer and 5'-GGTGCGCTCCTGGACGTA-3' for the reverse primer.

Male mice expressing the Macrophage EAS Induced Apoptosis transgene $\left(\mathrm{MaFIA}^{+/+}\right.$; coexpressing green fluorescent protein (GFP) and the dimerizer inducible suicide receptor under control of the endogenous CD115 promotor) on a pro-atherogenic Apo lipoprotein $\mathrm{E}$ deficient $\left(\mathrm{ApoE}^{-\mathcal{}}\right)$ background were obtained from the animal breeding facility at the Maastricht University. Dimerizer treated $\mathrm{ApoE}^{-/}$mice or solvent-treated $\mathrm{ApoE}^{-/} / /$ $\mathrm{MaFIA}^{+/+}$mice served as controls for the in vivo studies. Mice were fed regular chow diet (RM3; Special Diet Services [SDS], Essex, U.K.) or Western type diet (WTD; 0.25\% cholesterol; SDS). Drinking water supplemented with antibiotics $(60,000$ Units/L Polymixin B Sulfate [Sigma-Aldrich, St. Louis, MO, USA] and 100 $\mathrm{mg} / \mathrm{L}$ Neomycin [Sigma-Aldrich]) was introduced two weeks before the introduction of Western type diet. During experiments drinking water and food were provided ad libitum. During all experiments animals were housed in filter top cages. All in vivo studies were approved by the Maastricht University Ethics committee for animal experiments and performed according to the Dutch government guidelines for animal experiments. 


\section{Dimerizer}

Lyophilized AP20187 was dissolved in ice-cold pure (100\%) ethanol to obtain a $0.455 \mathrm{mM}(6.25 \mathrm{mg} / \mathrm{mL})$ stock solution, which was stored at $-20^{\circ} \mathrm{C}$ until further use. For in vitro experiments the stock solution was diluted in medium to a final concentration of $1-5 \mu \mathrm{M}$ in the culture medium with an end concentration of ethanol below $0.5 \%$. For intra-venous (iv) injections, the stock solution was diluted 25 -fold in water containing $4 \%$ ethanol, $10 \%$ poly(ethylene) glycol (PEG) 400, and 1.7\% Tween, resulting in an end concentration of $0.25 \mathrm{mg} / \mathrm{mL}$ AP20187. All intra-venous injections were administered within 30 minutes after preparation of the injection solution. The applied dose was $1 \mathrm{mg} / \mathrm{kg}$ body weight ( $150 \mu \mathrm{L} /$ mouse on average). ApoE ${ }^{-/}$ control animals received solvent containing AP20187 at the same dose as the MaFIA mice, $\mathrm{ApoE}^{-/} / / \mathrm{MaFIA}^{+/+}$controls received solvent-only injections. For the in vivo experiments in which osmotic minipumps (Alzet, Cupertino, CA, USA) were used, stock solution was diluted as before, however the administered dose dimerizer was 100 -fold lower $(0.01 \mathrm{mg} / \mathrm{kg})$.

\section{In vivo experiments}

IV injection atherosclerosis study. Ten week old $\mathrm{ApoE}^{--} / / \mathrm{MaFIA}^{+/+}$animals $(n=14)$ and $A p o E^{-}$animals ( $\left.n=13\right)$ were placed on a Western type diet. Two weeks after introducing the WTD animals received flow restricting collars around the right and left carotid arteries to induce atherosclerotic plaque formation, as described before ${ }^{33}$. Three weeks later, mice received an intravenous AP20187 tail vein injection every 72 hours for two weeks (final dose of $1 \mathrm{mg} / \mathrm{kg}$ body weight). Bodyweight was measured to adjust the dose according to the individual weight of each mouse and monitor the effect of the treatment. Mice were sacrificed 18 hours after the last injection with the dimerizer.

Osmotic minipump atherosclerosis study. Ten week old $\mathrm{ApoE}^{-/} / / \mathrm{MaFIA}^{+/+}$ animals $(n=8)$ and $A p o E^{-/}$animals $(n=8)$ were placed on a Western type diet. Two weeks after introducing WTD, semi-constrictive collars were placed bilaterally, at the common carotid arteries, to induce atherosclerotic plaque formation; simultaneously an osmotic minipump containing AP20187 with catheters attached to the perivascular site of the carotid artery was placed. Mice were sacrificed two weeks after implantation of the osmotic minipumps. 
Upon sacrifice the integrity and localization of the catheters was verified and animals with (suspected) malfunctions in the catheters or minipumps were excluded from the study.

Dynamics study. Ten week old $\mathrm{ApoE}^{-/} / / \mathrm{MaFIA}^{+/+}$animals $(n=16)$ and $\mathrm{ApoE}^{-/}$ animals $(n=8)$ were placed on a Western type diet for five weeks. Subsequently, MaFIA mice received an intravenous tail vein injection containing AP20187 (1 mg/kg body weight) every 72 hours for one or two weeks ( $n=8 /$ timepoint). Control $A p o E^{/-}$mice were processed similarly. Bodyweight was measured to adjust the dose according to the individual weight of each mouse. Mice were sacrificed 18 hours after the last injection with the dimerizer.

\section{In vitro experiments}

Macrophage culture. Bone marrow cells were isolated from $\mathrm{ApoE}^{-/} / / \mathrm{MaFIA}^{+/+}$ and $A \mathrm{AOE}^{-/}$mice ( $n=4 /$ genotype). Bone marrow cells were isolated from one femur and one tibia per mouse and pooled samples were cultured in RPMI1640 (Invitrogen) supplemented with 10\% fetal calf serum and 1929 conditioned medium (LCM) to generate bone marrow-derived macrophages (BMDM) as previously described ${ }^{34}$. Medium was replaced every three days and differentiated BMDM were used for in vitro assays after seven days.

Macrophage polarization. BMDM were incubated for 24 hours with LPS (100ng/mL), IFN- $\gamma(100 \mathrm{U} / \mathrm{mL})$, TNF- $\alpha(40 \mathrm{ng} / \mathrm{mL}), \mathrm{IL}-10$ (10 ng/mL), or IL-4 $(20$ $\mathrm{ng} / \mathrm{mL}$ ). Subsequently, mRNA was isolated using the Trizol (Invitrogen) method. $500 \mathrm{ng}$ total RNA was reverse transcribed using the iScript CDNA Synthesis Kit (BioRad, Veenendaal, the Netherlands). Quantitative PCR was performed using $10 \mathrm{ng}$ CDNA, $100 \mathrm{nM}$ of each primer, and SYBR Green (Invitrogen) in a total volume of $15 \mu \mathrm{l}$. Gene expression levels were corrected for glyceraldehyde 3-phosphate dehydrogenase (GAPDH) as housekeeping gene. Primer sequences are available upon request.

Apoptosis assay. Peritoneal macrophages isolated from $\mathrm{ApoE}^{--} / / \mathrm{MaFIA}^{+/+}$and ApoE $E^{-1}$ mice ( $n=5 /$ genotype) were cultured for 24 hours in RPMI-1640 (Invitrogen, Paisley, UK) supplemented with $10 \%$ fetal calf serum (FCS). Subsequently, AP20187 was added to the medium to a final concentration of 
$4.55 \mu \mathrm{M}$ and cells were incubated for 18 hours. Apoptosis was measured by immunohistochemically staining for cleaved caspase-3 and counterstaining with DAPI. Pictures were made (Leica DMIL fluorescence microscope) and overlays were analyzed with ImageJ Software.

\section{Histology}

Carotid arteries from $\mathrm{ApoE}^{/-} / / \mathrm{MaFIA}^{+/+}$and $\mathrm{ApoE}^{-/}$mice were collected, formalin-fixed and paraffin embedded. Subsequently, $4 \mu \mathrm{m}$ thick sections were stained with haematoxylin and eosin (H\&E) and lesion size and necrotic core area were determined in five sections per animal. Representative sections on separate slides were stained immunohistochemically with a macrophagespecific antibody (MAC-3; Becton \& Dickinson, NJ, USA) or with a smooth muscle cell-specific antibody ( $\alpha$-smooth muscle cell actin antibody; SigmaAldrich; St. Louis, MO, USA). Apoptosis was visualized using a terminal deoxytransferase dUTP nick-end labeling (TUNEL) kit (Roche; Woerden, Netherlands). Apoptotic cell content was determined by assessment of the TUNEL positive area per section. Collagen was visualized by Sirius-Red staining of representative slides.

Spleen and tibias were analyzed for the effects of the treatment on the hematopoiesis. After fixation for 24 hours in $1 \%$ paraformaldehyde spleen tissue was paraffin embedded and $4 \mu \mathrm{m}$ thick sections were cut for histological analysis. Tibias were fixated for 24 hours and subsequently decalcified for 2 weeks in decalcification buffer (PBS with 0.05 mM EDTA). Buffer was replaced every 3 days to obtain sufficient level of decalcification. Subsequently, tibias were paraffin embedded and $4 \mu \mathrm{m}$ thick sections were cut for RUNX2 and TRAP histological analysis. All histological analyses were performed, blinded, by an independent operator using Quantimet (Leica) with Qwin3 quantification software (Leica).

\section{M-CSF and cholesterol levels}

Blood samples were collected in tubes containing $5 \mu \mathrm{L}$ 0.5M EDTA and centrifugated for 10 minutes at $2100 \mathrm{rpm}$. Subsequently, plasma was isolated and used for M-CSF and total cholesterol measurement. M-CSF (ligand for CD115) was determined using the mouse M-CSF Quantikine ELISA kit (R\&D Systems). Total plasma cholesterol levels were measured using a colorimetric assay (CHOD-PAP, Roche, Mannheim, Germany). 


\section{Flow cytometry}

Upon sacrifice blood, spleen, bone marrow, and peritoneal leukocytes were collected. In blood absolute counts were obtained using BD Trucount tubes, according to the protocol supplied by the manufacturer (BD Biosciences). In short, Fc-receptor blocking antibody was added to the Trucount tubes. Subsequently, $50 \mu \mathrm{L}$ anti-coagulated whole blood was added and the tube was gently vortexed. After incubation for ten minutes, the antibody cocktail was added and incubated at room temperature in the dark for an additional 20 minutes. Finally, hypotonic lysis buffer was added and samples were measured on a FACS Canto II flow cytometer (BD Biosciences). Blood parameters were measured using flow cytometry for the leukocyte fraction and a Coulter counter for determining other variables. Red blood cell and platelet numbers were determined as well as hemoglobin and hematocrit levels.

Myeloid progenitors were measured in spleen and bone marrow. First, cells from spleen and bone marrow were stained with biotin-conjugated antibodies against lineage markers (CD5, CD45RA [B220], CD11b, Gr-1 [Ly6C/G], 7-4, and Ter-119; Miltenyi Biotec). Next, lineage positive cells were depleted using streptavidin conjugated magnetic beads (Miltenyi Biotech) and LS columns (Miltenyi Biotech). Lineage negative fractions were subsequently stained with antibodies for Sca-1, c-Kit (CD117), CD34, CD16/32, CD115, and lineage markers (CD5, CD45RA [B220], CD11b, Ly6C, Ly6G, Ter-119) and measured on a FACS Canto II (BD Biosciences).

Single cell suspensions were made from spleen by crushing the tissue over a $70 \mu \mathrm{m}$ cell strainer (BD Biosciences, NJ, USA). To measure mature leukocytes, erythrocytes in spleen and bone marrow were removed by incubation with hypotonic lysis buffer $\left(8.4 \mathrm{~g} \mathrm{NH}_{4} \mathrm{Cl}\right.$ and $0.84 \mathrm{~g} \mathrm{NaHCO}_{3}$ per liter distilled water). Non-specific Fc-receptor binding was blocked by the addition of anti-CD16/CD32 antibody (eBioscience, San Diego, USA). Antibodies used for these flow cytometry experiments are shown in supplemental table 1 . Samples and buffers were kept on ice throughout the experiment unless indicated otherwise. All measurements were performed on a FACS Canto II (BD Biosciences) and analysis of acquired data was performed using FACS Diva software (BD Biosciences). 


\section{Colony forming unit assay}

Bone marrow cells were isolated from one tibia and femur per mouse. Cells were counted twice using a Countess automated cell counter (Sigma-Aldrich) and the concentration was calculated for each sample. Per well 10,000 bone marrow cells were added to $2 \mathrm{~mL}$ methylcellulose medium with recombinant cytokines (MethoCult Medium, StemCell Technologies, Grenoble, France). For spleen 250,000 cells were added to $2 \mathrm{~mL}$ methylcellulose medium with recombinant cytokines (Stemcell Technologies). After incubation for 7 days $\left(37^{\circ} \mathrm{C} ; 5 \% \mathrm{CO}_{2}\right)$ the total number of colonies was quantified by an independent operator, and GM-CFU, E-CFU, G-CFU, and M-CFU colonies were specified based on morphology.

\section{Statistics}

Data are expressed as mean \pm SEM. To compare individual groups, 2-tailed Students t-test was used; non-parametric data were analyzed using MannWhitney $U$ test. All statistical analyses were performed using GraphPad Prism 5 software (GraphPad Software Inc, La Jolla, CA, USA) and p-values below 0.05 were considered statistically significant.

\section{Results}

Systemic treatment ablates plaque macrophages, but only affects lesion composition partially

As previously described, the MaFIA mice show weight loss due to the treatment with the dimerizer (data not shown) ${ }^{32}$, the weight loss is not present in solvent treated MaFIA mice or dimerizer treated $\mathrm{ApoE}^{/-}$controls. Pilot experiments showed the ablation of both monocytes and granulocytes after systemic treatment for 7 days using a dose of $1 \mathrm{mg} / \mathrm{kg}$ (figure $1 \mathrm{~A}+\mathrm{B}$ ). Effectiveness of the treatment on ablation of macrophages was verified in vitro using peritoneal macrophages isolated from $\mathrm{ApoE}^{-/} / / \mathrm{MaFIA}^{+/+}$and $\mathrm{ApoE}^{/-}$ mice. In the MaFIA peritoneal macrophages an almost complete induction of dimerizer-dependent cell death (>80\%) could be observed, while there was no apoptosis measurable in the control macrophages (figure 1C). For bone marrow derived macrophages in vitro no apoptosis could be induced. In depth analysis revealed that in vitro differentiation of BMDM resulted in downregulation of CD115 and thus diminished expression of the MaFIA suicide 
gene on these cells (supplemental figure $1 A+B$ ). This process was shown to be dependent on the M-CSF in the culture medium, indicating a negative feedback of M-CSF on the expression of the M-CSF receptor (CD115).

A
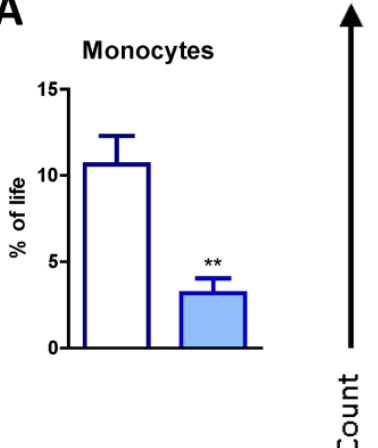

B

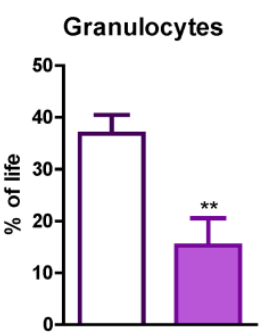

C
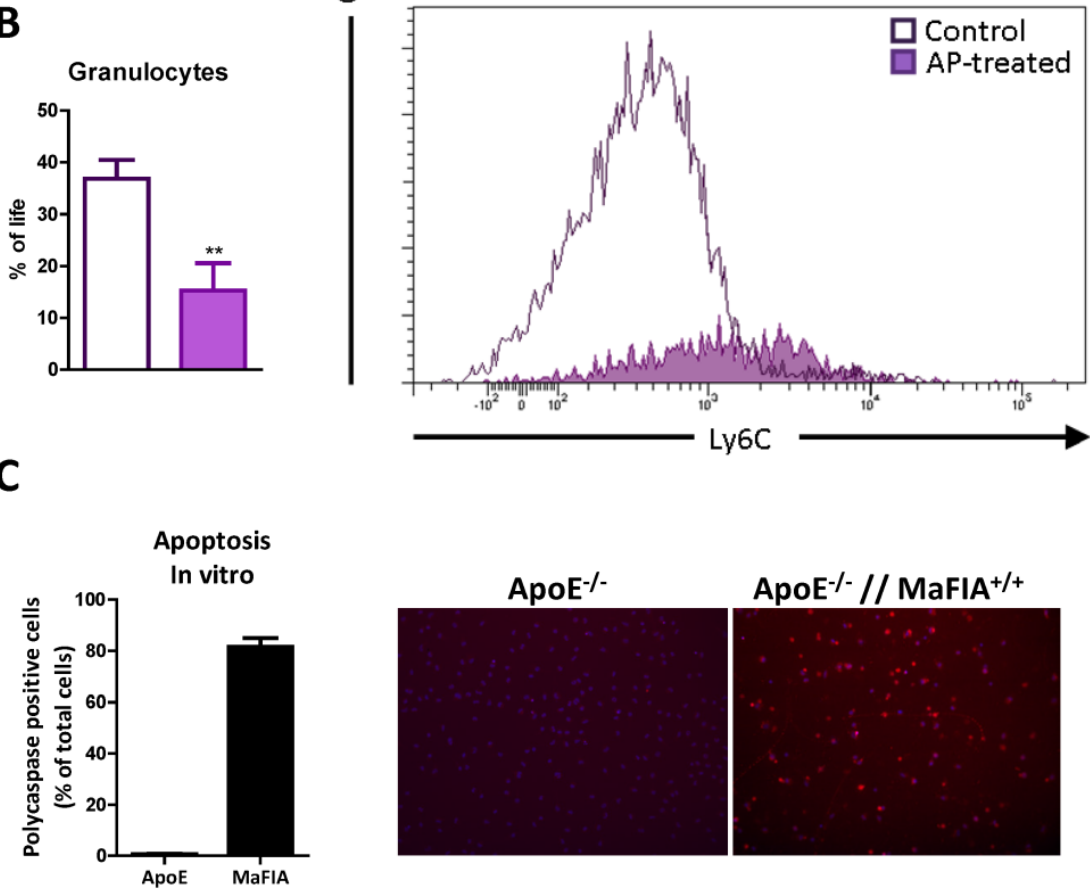

Figure 1 - In vitro and in vivo depletion of myeloid cells is functional

In vivo and in vitro depletion of monocytes, granulocytes, and macrophages using AP20187 is efficient. (A, B) Monocytes (A) and granulocytes (B) can be efficiently depleted in vivo after 7 days of treatment with $1 \mathrm{mg} / \mathrm{kg}$ intravenous injections of AP20187 (filled bar), compared to solvent-treated controls (open bar). (C) In vitro assays show highly efficient induction of apoptosis in peritoneal macrophages from $\mathrm{ApoE}^{-/-} / / \mathrm{MaFIA}^{+/+}$animals. Blue is DAPI (nuclei) and red is polycaspase (apoptosis). ${ }^{* * *} p<0.001$ 
To study the effect of ablating plaque resident macrophages on atherosclerosis development, we examined $\mathrm{ApoE}^{-/} / / \mathrm{MaFIA}^{+/+}$(MaFIA) mice treated with $1 \mathrm{mg} / \mathrm{kg} \mathrm{AP20187}$ via intra venous injections in the tail vein. Treatment with AP20187 did not influence plasma cholesterol levels (supplemental figure $2 A+B$ ), nor in plasma $M-C S F$ levels (supplemental figure 2D). At baseline, we observed no differences in collar-induced plaque characteristics between $\mathrm{ApoE}^{-/}$mice and (untreated) $\mathrm{ApoE}^{/-} / / \mathrm{MaFII}^{+/+}$mice (supplemental figure 3). After two weeks of treatment with AP20187, MaFIA mice did not have any changes in total plaque area (figure $2 \mathrm{~A}$ ) nor in necrotic core size (figure 2B) compared to AP20187 treated $\mathrm{ApoE}^{-/}$mice. Plaque area did increase over the course of the two week treatment period compared to baseline (dashed line; figure $2 \mathrm{~A}+\mathrm{B}$ ). Collagen was decreased significantly in $\mathrm{ApoE}^{-\alpha} / / \mathrm{MaFIA}^{+/+}$mice compared to $\mathrm{ApoE}^{-/}$controls (figure $2 \mathrm{C}$ ). As expected an ablation of plaque macrophages was observed in the dimerizer-treated $\mathrm{ApoE}^{-\alpha} / / \mathrm{MaFIA}^{+/+}$mice compared to $\mathrm{ApoE}^{-/}$controls (figure 2D). However, the increased apoptosis of macrophages did not result in any effects on necrotic core, indicating that this reduction could possibly be caused by decreased monocyte migration towards the atherosclerotic lesion. Surprisingly, we also noted a significant reduction in smooth muscle cells (SMC) in lesions from $\mathrm{ApoE}^{/-} / / \mathrm{MaFIA}^{+/+}$mice compared to control $\mathrm{ApoE}^{-/}$mice (figure $2 \mathrm{E}$ ). To verify whether this was related to increased apoptosis of SMCs we performed a TUNEL staining. An almost 4-fold increase in SMC apoptosis could be observed indicating that also smooth muscle cells are directly or indirectly affected by the systemic treatment with AP20187 in vivo (figure 2F). As there were no effects observed on smooth muscle cells in the control animals, it was not solvent-induced apoptosis of SMCs, but a specific reaction to the dimerizer treatment. Next, it could be noted that lesions from MaFIA mice had less pronounced lipid cores compared to wild type animals. However, total lipid core was unchanged between $\mathrm{ApoE}^{-/} / / \mathrm{MaFIA}^{+/+}$and $\mathrm{ApoE}^{-/}$mice (data not shown). 
A

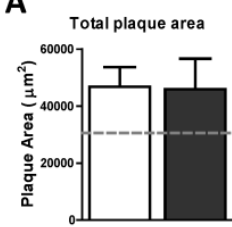

E

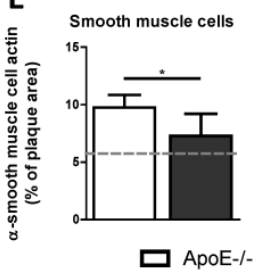

B

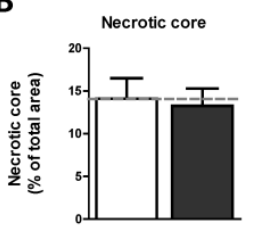

$\mathbf{F}$

SMC apoptosis

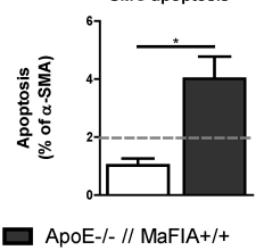

C

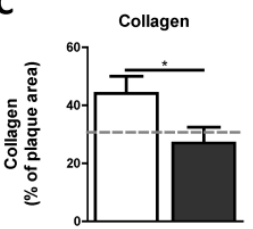

D

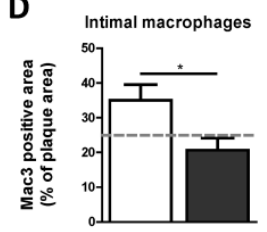

H

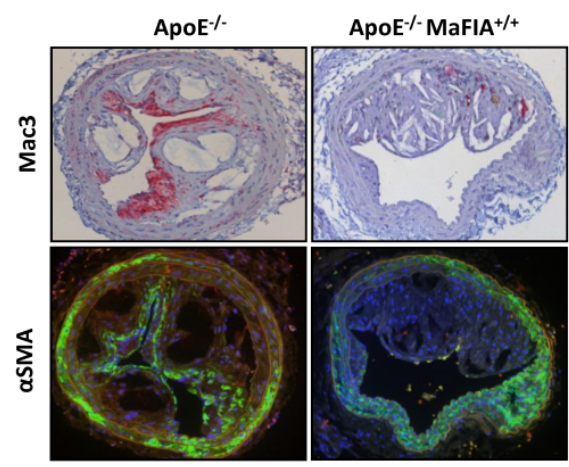

Figure 2 - Systemic treatment with AP20187 reduces plaque macrophage content

(A - C) In vivo treatment with $1 \mathrm{mg} / \mathrm{kg}$ AP20187 via intravenous tail vein injections does not affect total plaque area (A) and necrotic core area (B), while collagen content decreases (C) in $\mathrm{ApoE}^{-/-} / / \mathrm{MaFIA}^{+/+}$animals. (D, E) Intimal macrophages content (D) and smooth muscle cells (E) are reduced in $\mathrm{ApoE}^{-/-} / / \mathrm{MaFIA}^{+/+}$animals upon AP20187 treatment. (F) This decreased number of SMCs is accompanied by an increased SMC apoptosis in $\mathrm{ApoE}^{-/-} / / \mathrm{MaFIA}^{+/+}$animals. (G) Overview of the experimental setup for the in vivo treatment with AP20187. (H) Representative pictures of macrophage (Mac3) and alpha smooth muscle cell actin ( $\alpha \mathrm{SMA}$ ) staining for $\mathrm{ApoE}^{-/}$ and $\mathrm{ApoE}^{-/-} / / \mathrm{MaFIA}^{+/+}$animals. White bars are $\mathrm{ApoE}^{-/-}$controls and black bars are $\mathrm{ApoE}^{-/-} / /$ MaFIA $^{+/+}$animals. $* p<0.05$

Myeloid cells are depleted systemically; however recover after sustained treatment

Systemic treatment $(1 \mathrm{mg} / \mathrm{kg})$ resulted in decreased numbers of CD45 positive leukocytes in the circulation of AP20187 treated $\mathrm{ApoE}^{-/} / / \mathrm{MaFIA}^{+/+}$mice compared to $A p O E^{-/}$mice after one week of treatment. This was mainly contributable to the decreased number of myeloid cells in MaFIA mice compared to controls. Contrary to our expectations, total leukocyte numbers recovered after two weeks of continuous treatment with AP20187 to levels equal, or even slightly increased, compared to $A p E^{/-}$control mice. However, leukocyte composition was clearly affected, lymphocytes were affected only 
partially by the AP20187 treatment; this was shown by unaltered numbers of CD3 positive T lymphocytes (data not shown). B lymphocytes (B220+ cells) were decreased in MaFIA mice after already 1 week of treatment and did not recover after two weeks of treatment (data not shown).

Total myeloid cells were affected more drastically and showed an increase after prolonged treatment of two weeks. Monocytes (CD11b+/Ly6G-) and surprisingly also granulocytes (CD11b+/Ly6G+) were efficiently depleted in the circulation of the $\mathrm{ApoE}^{-/} / / \mathrm{MaFIA}^{+/+}$compared to $\mathrm{ApoE}^{-/}$mice after the first week of treatment with AP20187 (figure 3A+B). In contrast to our expectations, monocytes and granulocytes in MaFIA mice recovered to baseline values after continuous treatment for two weeks compared to short (one week) treatment (figure $3 \mathrm{~A}+\mathrm{B}$ ). We also noted the expansion of a $\mathrm{CD}_{11} \mathrm{~b}^{+} / \mathrm{Ly}_{6 \mathrm{G}}{ }^{\text {mid }}$ myeloid subset, not being (mature) monocytes or granulocytes, after one and two weeks of treatment with AP20187 (figure 3C). The amount of Ly $6 \mathrm{C}^{\text {high }}$ monocytes recovered faster than the Ly6C monocytes (figure 3D) upon the depletion after 1 week of dimerizer treatment. This could be indicative of expanded production or release of Ly $6 \mathrm{C}^{\text {high }}$ monocytes from bone marrow and spleen in the AP20187 treated MaFIA mice.

As the massive depletion of granulocytes was unexpected we analyzed the expression of both the GFP reporter and also the M-CSF receptor (CD115) on these cells. Monocytes were $\mathrm{CD} 115^{\text {high }}$ as expected, however also granulocytes showed low to intermediate levels of the M-CSF receptor. The activity of the CD115 promotor in granulocytes was confirmed by the GFP signal, which was comparable to the GFP level in monocytes (figure 3F). Based on the GFP levels we could also determine that a subset of B-cells has low expression of the construct, explaining the limited effects observed on circulating B-cells (data not shown). Treatment with AP20187 resulted in depletion of CD115 positive cells, however GFP expression was still measurable in CD115 negative cells (figure 3F), which could be indicative of posttranslational regulation of the M-CSF receptor expression. 
A

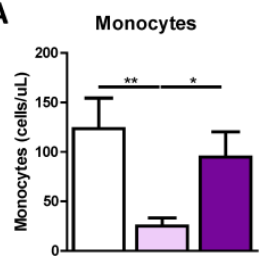

B

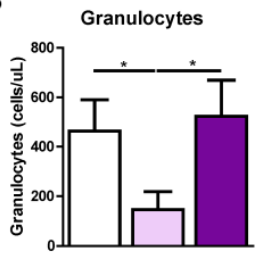

C

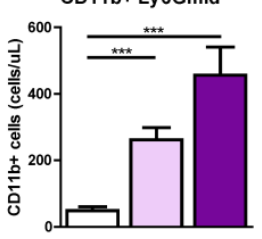

D

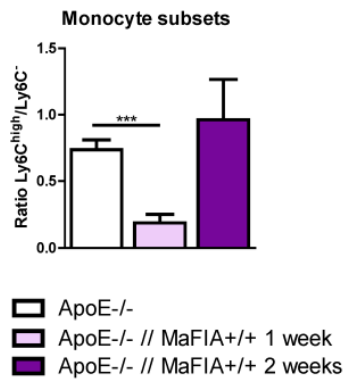

E
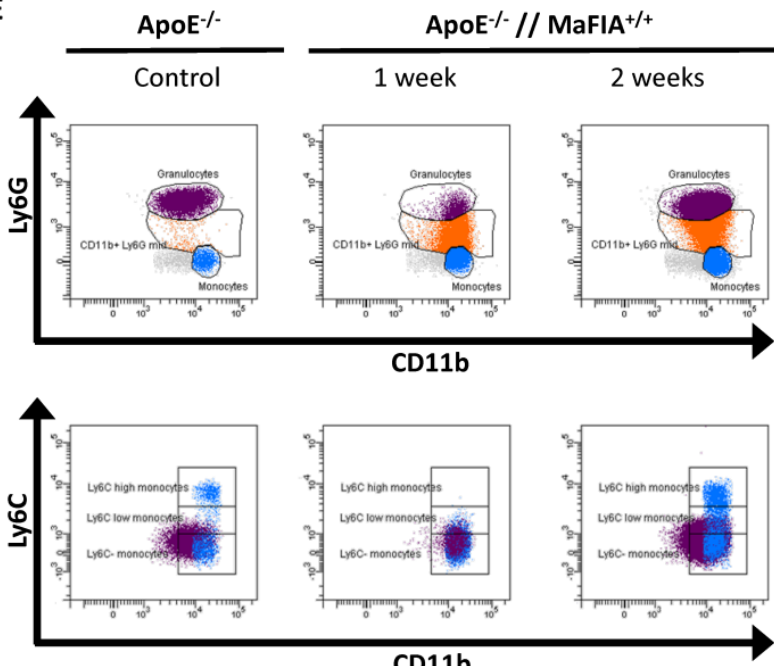

CD11b
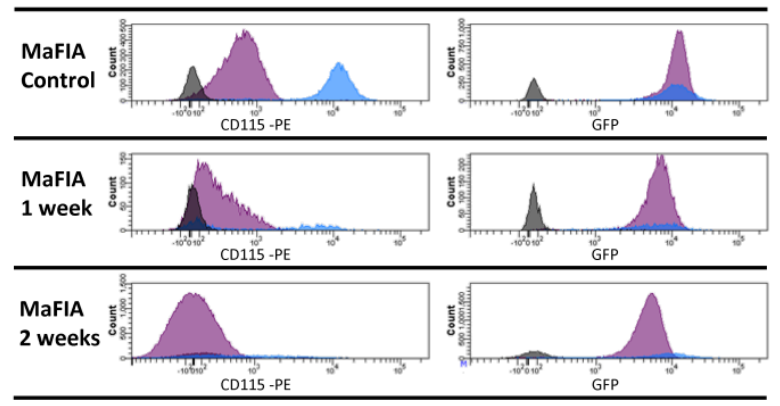

Monocytes

Granulocytes

Control

Figure 3 - Sustained treatment with AP20187 results in disturbed leukocyte pattern

(A, B) Treatment with AP20187 results in initial depletion of monocytes (A) and granulocytes (B) after 1 week, but apparent recovery after two weeks of treatment. (C) Leukocyte patterns are disturbed upon AP20187 treatment and a novel $\mathrm{CD} 11 \mathrm{~b}^{+} / \mathrm{Ly}_{6 \mathrm{G}}{ }^{\text {mid }}$ population increases over time. (D) Monocyte subset (Ly6C $\mathrm{C}^{\text {high }} / \mathrm{Ly}_{6 \mathrm{C}}$ ) ) ratio show a pattern that is similar to total monocytes and granulocytes, with initial depletion and subsequent recovery. (E) Representative dot plots of monocytes and granulocytes (top panels) and monocyte subsets (bottom panels). (F) Representative histograms showing CD115 (M-CSF receptor) and GFP (promotor activity) levels in monocytes, granulocytes, and control cells. White bars (controls, light purple bars (1 week treatment), and dark purple bars (2 weeks treatment). ${ }^{*} p<0.05,{ }^{* *} p<0.01, * * * p<0.001$ 
Unexpectedly, analysis of splenic leukocytes revealed a significant and persistent increase in monocytes (figure 4A) and granulocytes (figure 4B) after 1 and 2 weeks of treatment. Also $\mathrm{CD} 11 \mathrm{~b}^{+} / \mathrm{Ly}_{6 \mathrm{G}}{ }^{\text {mid }}$ cells were massively expanded in the spleen (figure $4 \mathrm{C}$ ). Flow cytometry also revealed that the monocyte expansion was $\mathrm{CD} 115$ independent, as $\mathrm{CD} 115^{+}$monocytes were almost completely ablated (figure 4D), and thus all remaining monocytes were CD115 negative.

A

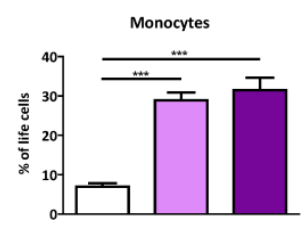

E

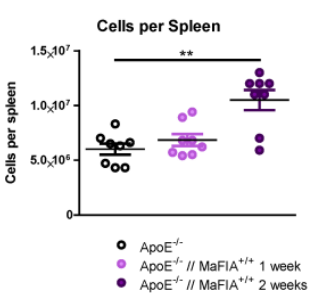

H

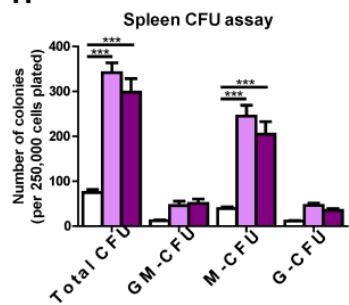

B

$\mathbf{F}$
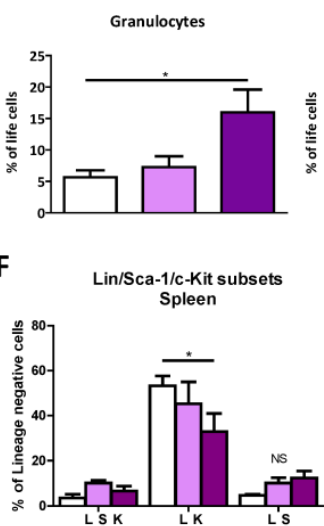

I

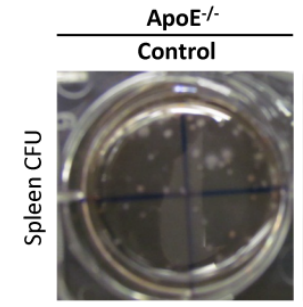

C

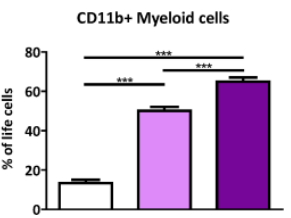

D

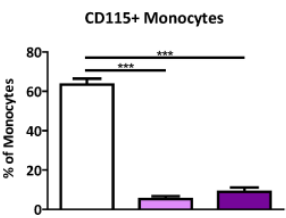

G GMP

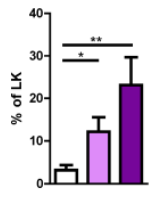

$\square$ ApoE-I

$\square$ ApoE-l- // MaFIA $+1+1$ week

$\square$ ApoE-/- // MaFIA+/+ 2 weeks

ApoE $^{\%} / / / \mathrm{MaFIA}^{+/+}$

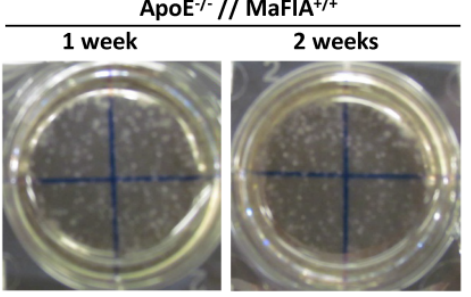

Figure 4 - Spleen composition and function is perturbed by $\mathrm{CD}_{115^{+}}$cell depletion

(A - C) Treatment of MaFIA mice with $1 \mathrm{mg} / \mathrm{kg}$ AP20187 results in increased monocytes (A), granulocytes (B), and $\mathrm{CD} 11 \mathrm{~b}^{+} / \mathrm{Ly}_{6 \mathrm{G}}{ }^{\text {mid }}$ myeloid cells (C) in the spleen. (D) These splenic monocytes are however predominantly CD115 negative. (E) Total spleen cellullarity is significantly increased after sustained AP20187 treatment in MaFIA mice compared to controls. $(\mathbf{F}, \mathbf{G})$ Flow cytometry analysis of the spleen indicated that Lineage ${ }^{-} / \mathrm{Sca}-1^{-} / \mathrm{C}_{-} \mathrm{Kit}^{+}$(LK) cells were reduced upon treatment $(\mathbf{F})$, however within this subset granulocyte/macrophage progenitors (GMP) (G) were increased. (H, I) Colony forming unit (CFU) assay showed that total number of colonies and macrophage colony forming units (M-CFU) were increased after 1 and 2 weeks of treatment in MaFIA mice, as could also be observed in representative pictures of CFU assay (I). * $p<0.05, * * p<0.01, * * * p<0.001$ 


\section{Myelopoiesis shifts to spleen after AP20187 treatment}

As there was an unexpected increase in myeloid cells in the circulation (figure 3 ) and spleen (figure 4A-D), hematopoiesis was analyzed more in depth. As the spleen was enlarged (data not shown) and splenic cell content was increased (figure $4 \mathrm{E}$ ), myelopoiesis was analyzed in the spleen to determine whether extramedullar hematopoiesis did occur in these treated MaFIA mice. In spleen, no effects on the LK fraction could be observed after one week of treatment. Two weeks treatment did result in a significant decrease of this progenitor subset (figure 4F). More in depth analysis did show that the decrease in LK cells was accompanied by an increase in GMP cells after both one and two weeks of treatment (figure $4 \mathrm{G}$ ). Indeed, splenic myelopoiesis was dramatically increased after 1 and 2 weeks of treatment with AP20187. Total CFU was more than 3fold increased, which was almost completely attributable to increased numbers of macrophage colony forming units (M-CFU), while GM-CFU and GCFU were not significantly increased (figure $4 \mathrm{H}$ ).

Macroscopically, it could be noted that there was a clear distinction between the bones (femur and tibia) from $\mathrm{ApoE}^{--}$mice and $\mathrm{ApoE}^{-/} \mathrm{MaFIA}^{+/+}$ mice treated with AP20187. MaFIA mice had paler bone marrow which already indicated effects on bone marrow composition (figure $5 A$ ), which was confirmed by the decreased number of cells in the bone marrow (figure $5 B$ ). When myelopoiesis in bone marrow was analyzed by colony forming unit (CFU) assays it could be observed that there was a decrease in total CFU after 1 week of treatment, while after two weeks of treatment with the dimerizer levels normalized (figure 5C). These effects were similar to the effects observed in blood regarding monocyte and granulocyte numbers (figure 3 ). There were no specific subsets that were responsible for the decreased number of CFUs in the bone marrow compartment, as all subsets showed equal responsiveness to the treatment after one and two weeks of treatment (figure 5C). To confirm these results from the CFU assays, flow cytometry was performed to determine progenitors in bone marrow compartment. It could be observed that Lineage /Sca-1/c-Kit ${ }^{+}$(LK) cells were significantly diminished in bone marrow after one week of treatment (figure 5D), which is in line with the effects observed in the CFU assays. Within this LK fraction the relative percentage of granulocyte/macrophage progenitors (GMP) was increased, indicative of a relatively small decrease or even expansion of this progenitor subset in the bone marrow (figure $5 \mathrm{E}$ ). Overall, it could be shown that in spite of treatment 
myelopoiesis did expand. Most likely this is a compensatory effect for the depleted mature myeloid cells in the periphery. Especially, the splenic hematopoiesis is thought to be responsible for the effects observed in blood and spleen regarding the recovery of myeloid cells after prolonged treatment.

\section{Systemic treatment results in bone marrow osteoclast depletion}

As we observed massive extramedullar hematopoiesis in MaFIA mice treated systemically (i.v. injections), we sought out to investigate which mechanism is responsible for the peripheral phenotype observed. Since we observed a shift in hematopoiesis from the bone marrow to the splenic compartment, we wanted to analyze the effect of the dimerizer (AP20187) treatment on cells involved in maintaining normal bone marrow niches. Staining for osteoclasts in decalcified femur sections showed that osteoclast levels were mildly reduced in MaFIA mice (supplemental figure 4).

A

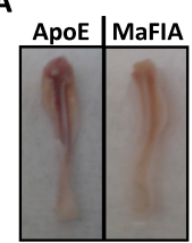

D
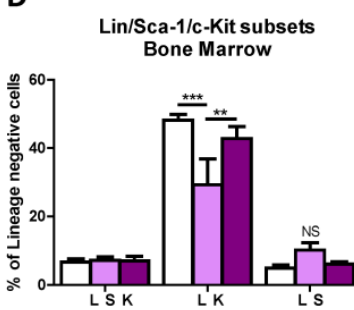

$\square$ ApoE-/-

$\square \mathrm{ApoE}-/-/ / \mathrm{MaFIA}+/+2$ weeks
B

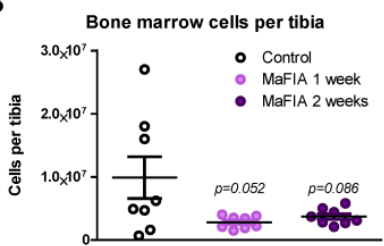

E

\section{$\mathbf{F}$}

C Bone Marrow CFU assay

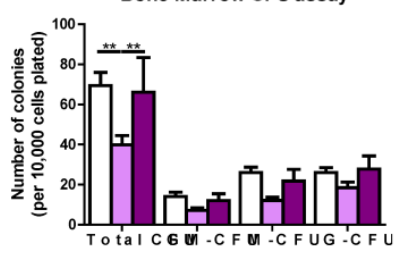

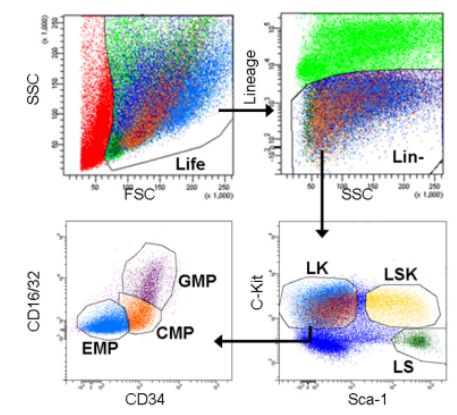

Figure 5 - Stromal hematopoiesis is decreased in treated MaFIA mice

(A) Femurs from AP20187 treated MaFIA mice were visibly paler than femur from control animals. (B) Total cells per bone were decreased in MaFIA mice. (C) Colony forming unit (CFU) assay showed that total number of colonies (and subsets) were decreased after 1 week of treatment, but recovered after two weeks of sustained treatment in MaFIA mice. (D, E) Flow cytometry analysis of the bone marrow indicated that Lineage $/ \mathrm{Sca}-1 / \mathrm{c}-\mathrm{Kit}^{+}$(LK) cells were reduced upon treatment (D), however within this subset granulocyte/macrophage progenitors (GMP) (E) were increased. (F) Representative dot plots indicating relevant gating strategy. ${ }^{* *} p<$ $0.01,{ }^{* * *} p<0.001$ 


\section{Local treatment with AP20187 accelerates atherogenesis}

To exclude any systemic effects on atherosclerosis development, we performed a second atherosclerosis study in which animals received a collar around the carotid arteries combined with an osmotic minipump with catheters attached to the perivascular side of the carotid arteries near the collar. The dimerizer AP20187 was constantly delivered for two weeks at a dose of $0.01 \mathrm{mg} / \mathrm{kg}$ body weight, which did not affect plasma cholesterol (supplemental figure $2 \mathrm{C}$ ). Analysis of the carotid arteries after two weeks of treatment showed that total plaque area was over two-fold increased when the animals were treated continuously and locally compared to treated $\mathrm{ApoE}^{-1-}$ controls (figure 6A). Necrotic core (figure 6B) and collagen content (figure 6C) were equally expanded in the treated MaFIA mice. As expected, macrophages were not increased in the plaques from MaFIA mice compared to the controls despite the massively expanded plaque size (figure 6D). Intra-plaque smooth muscle cells were in contrast to the systemic study not affected by the local treatment with the dimerizer (figure $6 \mathrm{E}+\mathrm{F}$ ). Analysis of circulating leukocytes did not reveal any significant differences between $\mathrm{ApoE}^{-/-} \mathrm{MaFIA}^{+/+}$mice and $A p o E^{-/}$controls (supplemental figure 5).

A

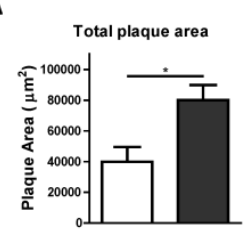

D

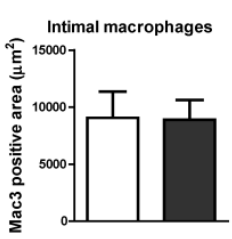

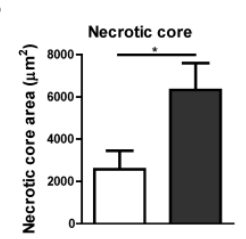

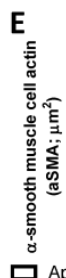

Smooth muscle cells

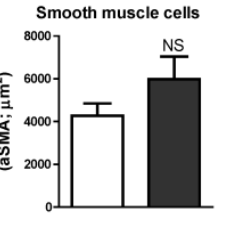

C

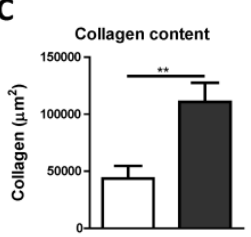

$\mathbf{F}$

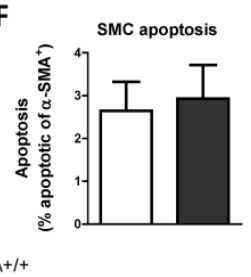

G

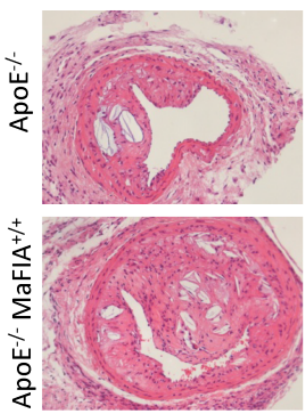

Figure 6 - Local treatment to the atherosclerotic lesion increases lesion formation

(A) Local treatment with AP20187 results in aggravated atherosclerosis formation in MaFIA mice. (B, C) Local treatment does not affect necrotic core (B), and plaque collagen content (C) in MaFIA mice compared to controls. (D) Intimal macrophage content is reduced upon treatment. (E, F) However, smooth muscle cell (SMC) content (E), and SMC apoptosis (F) are unaffected by local treatment. (G) Representative pictures of haematoxylin and eosin (H\&E) stained lesions from control and MaFIA mice. ${ }^{*} p<0.05$ 


\section{Discussion}

The MaFIA model for induction of $\mathrm{CD}_{115^{+}}$cell apoptosis in $A p o E^{-/}$proatherosclerotic mice was used for this study. Cells of the myeloid lineage express an inducible suicide gene under transcriptional control of the CD115promoter ${ }^{32}$. Apoptosis is prompted by administration of AP20187 which results in dimerization of extracellular FK domains and subsequently their cytoplasmic FAS domains triggering the extrinsic pathway of cell apoptosis ${ }^{32}$.

Macrophages are essential in atherosclerotic lesion development and progression, and contribute to clinical outcomes by destabilizing the atherosclerotic lesion ${ }^{35,36}$. Macrophages have an important role throughout atherogenesis, from early intimal thickening until advanced rupture-prone atheromas ${ }^{37}$. However, it has been difficult to unravel the role of plaque macrophages, next to circulating myeloid cells, in relation to the progression of the lesions. Here, we show that specific depletion of these cells, in a FAS dependent manner, exposed both local and systemic effects which contribute differentially to atherosclerosis, but also to the conservation of normal hematopoiesis.

Lesion composition in general was not affected by systemic ablation of macrophages, despite a substantial reduction of plaque resident macrophages. Vascular smooth muscle cells (VSMC) were partially reduced by the whole body treatment. However, it has been shown that VSMC which are in contact with an atherosclerotic lesion can express the M-CSF receptor ${ }^{38,39}$, explaining the depletion of these cells in vivo. These smooth muscle cells show also other characteristics of the monocyte/macrophage lineage such as phagocytic capacity and even the transdifferentiation into foam cells ${ }^{38}$. Nevertheless, the increased apoptosis of VSMC and macrophages did not contribute to any effects on atherosclerotic lesion phenotype.

Peripheral leukocyte analysis revealed a dramatic decrease in total leukocytes after seven days of AP20187 treatment. This reduction was almost completely contributable to ablation of myeloid cells, both monocytes/macrophages and granulocytes, with minor effects on Blymphocytes. The effects on granulocytes and B-cells were in part unexpected, however could be explained by the role of PU.1 in controlling M-CSF receptor expression ${ }^{40,41}$. PU.1 was already shown to be involved in myeloid cell development and B-cell development in a PU.1 knock-out model ${ }^{42}$. The ablation of granulocytes, but also B-cells, can be related to the PU.1 dependent 
transcription of the M-CSF receptor and thus also transcription of the MaFIA transgene. The transcription, and even expression, of the M-CSF receptor on both B-cells and granulocytes has only been scarcely described so far, but could have profound impact on the current knowledge on myelopoiesis and cell maturation.

Systemic ablation of M-CSF receptor (CD115) positive macrophages and monocytes was shown to result in disturbed myelopoiesis with increased extra-medullar hematopoiesis and the release of immature myeloid cells into the circulation. The production and release of progenitors in the bone marrow is amongst others controlled by bone marrow monocytes, macrophages, and osteoclasts, which are considered to be related to the myeloid lineage ${ }^{43}$. Osteoclasts respond to similar cytokines as macrophages, and also express comparable surface markers, including the M-CSF receptor ${ }^{44}$. We were able to show that in vivo treatment with AP20187 results in decreased numbers osteoclasts. As macrophages as well as osteoclasts are ablated upon AP20187 treatment, the relevant contribution of each these cell types to the observed neutrophilia cannot be directly derived from these data ${ }^{44-46}$. However, in a GCSF mobilization study Winkler et al argued that bone marrow macrophages are the most important cell type in regulating stromal release ${ }^{45}$. Furthermore, osteoclast activation is associated with decreased stromal release ${ }^{47}$, yet osteoclast activation is increased under inflammatory conditions ${ }^{48}$. Osteoclast depletion may also be responsible for the observed effects on B-lymphocytes as the development of mature B-cells also is regulated by these cells in the bone marrow ${ }^{49}$. Overall, systemic AP20187 treatment was shown to have profound effects on myelopoiesis, which could, at least in part, be explained by depletion of cells responsible for maintaining normal bone marrow niches.

As the unanticipated effects observed on the myelomonocytic cells after systemic dimerizer administration could well have masked local effects of macrophage ablation, we analyzed plaque-specific effects in a separate study. The dimerizer was administered locally to the carotid arteries via catheterized osmotic minipumps. Site-restricted administration of the dimerizer was shown not to result in any significant systemic effects. In the atherosclerotic lesion we did observe a completely inhibited expansion of the number of intimal macrophages. In contrast to systemic administration of the dimerizer, plaque size was increased more than two-fold in animals treated locally. As macrophage content was similar between the treated MaFIA mice and 
controls, the plaque and necrotic core expansion were most likely caused by the immediately induced cell death of newly infiltrating monocytes. As monocytes are not ablated peripherally in mice treated locally, more monocytes will migrate towards the inflammatory locus ${ }^{50}$. Hereby, more cells will enter the lesion, where AP20187-dependent apoptosis is induced. Due to an inhibited expansion of mature macrophages, and thus decreased efferocytosis, these apoptotic monocytes will be cleared less efficiently giving rise to secondary necrosis and progressive development of the necrotic core and total plaque. Taken together, our data confirm the current paradigm that influx of monocytes might be more important than reduction of plaque macrophages via efflux of apoptosis for controlling atherosclerotic plaque development ${ }^{50-53}$. Another explanation may lie in the mildly shifted Ly $6 \mathrm{C}^{\text {high }}$ to Ly6C $\mathrm{C}^{-/ 10 w}$ monocyte balance which may have favored the differentiation into classically activated pro-inflammatory macrophages ${ }^{54-56}$.

Overall, we can conclude that macrophages, and other M-CSF receptor positive cells, are critical controllers of many processes essential to atherogenesis, including hematopoiesis. Depletion of macrophages will result in disturbed physiology indicative of the essential role of myeloid cells in maintaining health and reducing plaque development.

\section{Acknowledgements}

This work was performed within the framework of the Centre for Translational Molecular Medicine (CTMM; http://www.ctmm.nl), project CIRCULATING CELLS [grant number 01C-102] and was supported by the Netherlands Heart Foundation. The dimerizer (AP20187) used to induce ablation of CD115 positive cells in the in vitro and in vivo experiments was a kind gift from Ariad Pharmaceuticals (MA, USA). 


\section{Supplemental figures}

A

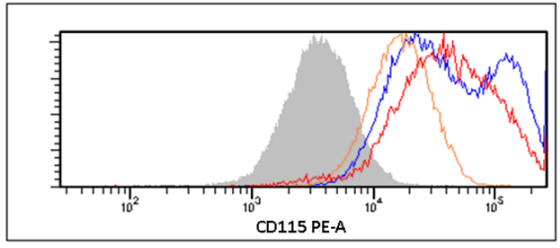

B

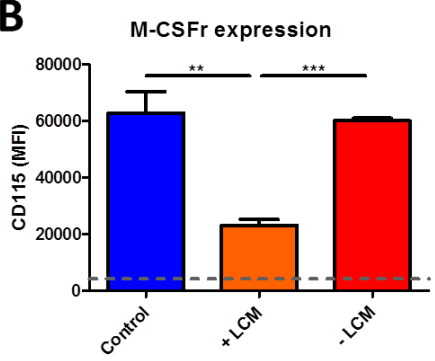

Supplemental figure 1 - M-CSF reduces CD115 expression on bone marrow derived macrophages

(A, B) M-CSF containing medium (LCM) results in down regulation of CD115 (M-CSF receptor) within 24 hours compared to M-CSF free cultured bone marrow derived macrophages.

A

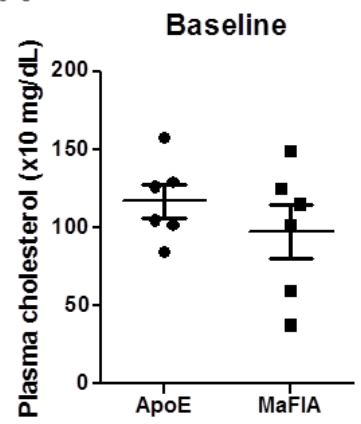

C

Local

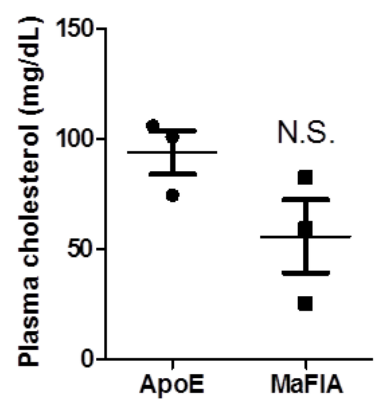

B

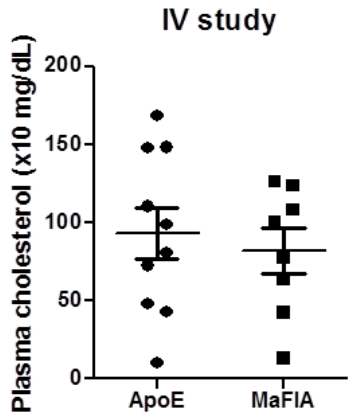

D

Plasma M-CSF levels

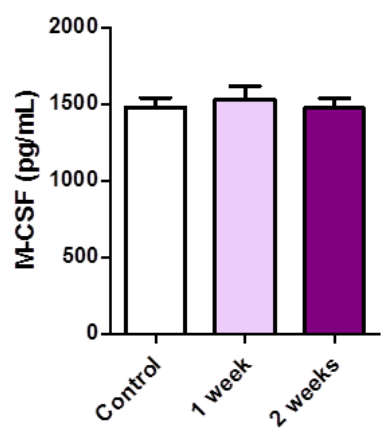

Supplemental figure 2 - Plasma cholesterol is not affected by AP20187 treatment

(A, B, C) Plasma cholesterol is not affected at baseline (A), after intravenous treatment with AP20187 (B), or after local treatment (minipump) with AP20187 (C) of MaFIA mice compared to controls. (D) Plasma M-CSF levels after 1 and 2 weeks intravenous AP20187 administration. 
A

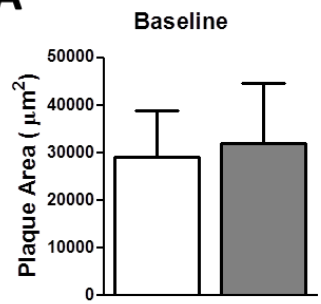

D

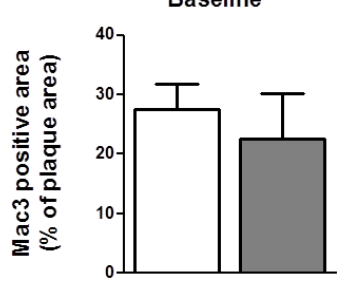

B

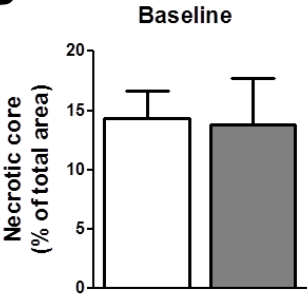

$\mathbf{E}$

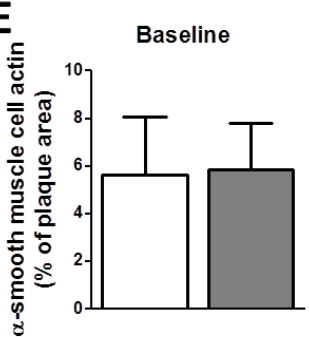

C

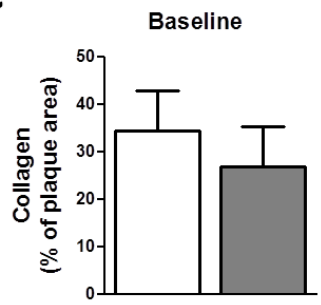

$\mathbf{F}$

Baseline

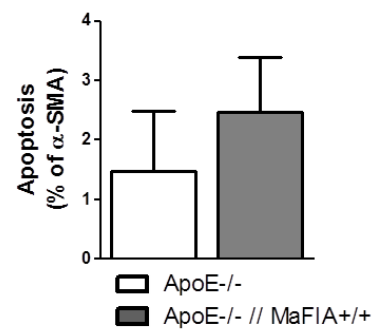

Supplemental figure 3 - At baseline no differences in plaque size and morphology could be detected

At baseline no differences in total plaque area (A), necrotic core (B), collagen content (C), intimal macrophage content (D), smooth muscle cell content (E), and apoptosis (F) could be detected between control animals and AP20187 treated MaFIA mice.

A

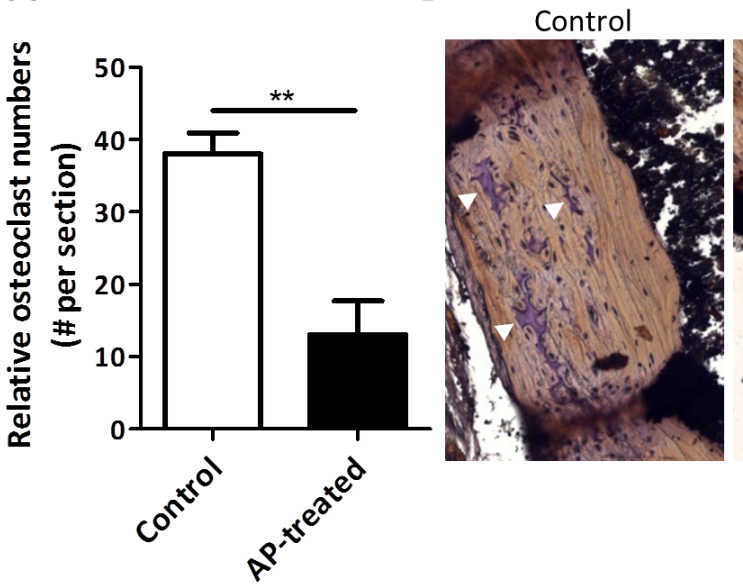

AP-treated

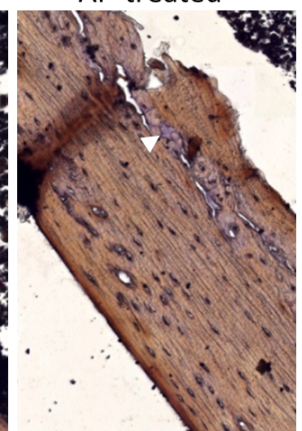

Supplemental figure 4 - Osteoclasts are depleted from the bone upon AP20187 treatment (A) Osteoclasts were stained and quantified in the bones of solvent-treated (control) animals (white bar) and dimerizer (AP) treated animals (black bar). A clear reduction in the number of osteoclasts could be shown in the AP-treated animals. (B) Representative pictures of bones from control and AP-treated animals. Osteoclasts are stained in purple, arrowheads indicate the positive cells. Note that in the AP-treated animals the staining is less pronounced than in the control animals. $* p<0.05$ 


\section{A \\ B}

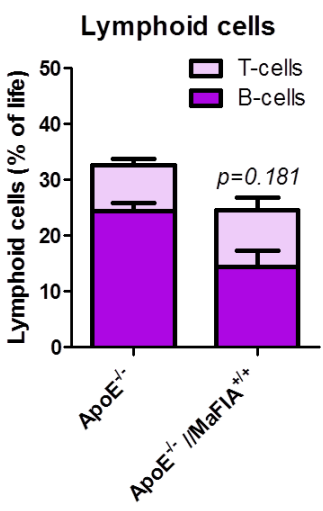

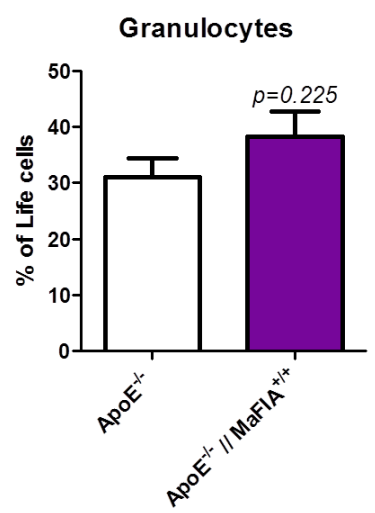

C

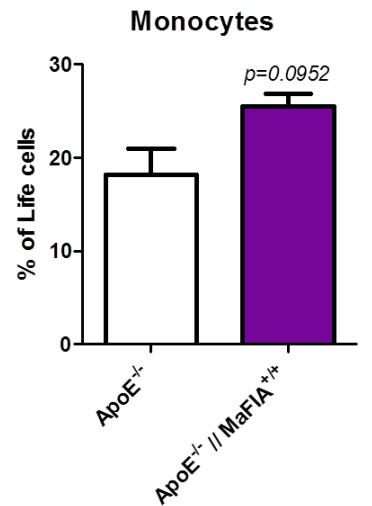

Supplemental figure 5 - Local treatment does not induce systemic disturbances in leukocyte composition

After local treatment with AP20187, no differences could be detected in circulating lymphocytes (A), granulocytes (B), and monocytes (C) between control animals and AP20187 treated MaFIA mice. 


\section{References}

1. Lusis AJ. Atherosclerosis. Nature. 2000;407(6801):233-241.

2. Descamps D, Le Gars M, Balloy V, et al. Toll-like receptor 5 (TLR5), IL-1beta secretion, and asparagine endopeptidase are critical factors for alveolar macrophage phagocytosis and bacterial killing. Proc Natl Acad Sci U S A. 2012;109(5):1619-1624.

3. Mirza R, DiPietro LA, Koh TJ. Selective and specific macrophage ablation is detrimental to wound healing in mice. Am J Pathol. 2009;175(6):2454-2462.

4. Liao X, Sluimer JC, Wang Y, et al. Macrophage autophagy plays a protective role in advanced atherosclerosis. Cell Metab. 2012;15(4):545-553.

5. Van Vre EA, Ait-Oufella H, Tedgui A, Mallat Z. Apoptotic cell death and efferocytosis in atherosclerosis. Arterioscler Thromb Vasc Biol. 2012;32(4):887-893.

6. Quillard T, Tesmenitsky Y, Croce K, et al. Selective inhibition of matrix metalloproteinase-13 increases collagen content of established mouse atherosclerosis. Arterioscler Thromb Vasc Biol. 2011;31(11):2464-2472.

7. Panizzi P, Swirski FK, Figueiredo JL, et al. Impaired infarct healing in atherosclerotic mice with Ly-6C(hi) monocytosis. J Am Coll Cardiol. 2010;55(15):16291638.

8. Krohn R, Raffetseder $U$, Bot $I$, et al. Y-box binding protein-1 controls CC chemokine ligand-5 (CCL5) expression in smooth muscle cells and contributes to neointima formation in atherosclerosis-prone mice. Circulation. 2007;116(16):18121820.

9. Swirski FK, Pittet MJ, Kircher MF, et al. Monocyte accumulation in mouse atherogenesis is progressive and proportional to extent of disease. Proc Natl Acad Sci U S A. 2006;103(27):10340-10345.

10. Nahrendorf M, Swirski FK, Aikawa E, et al. The healing myocardium sequentially mobilizes two monocyte subsets with divergent and complementary functions. J Exp Med. 2007;204(12):3037-3047.

11. Swirski FK, Weissleder R, Pittet MJ. Heterogeneous in vivo behavior of monocyte subsets in atherosclerosis. Arterioscler Thromb Vasc Biol. 2009;29(10):14241432.

12. Chapman CM, Beilby JP, McQuillan BM, Thompson PL, Hung J. Monocyte count, but not C-reactive protein or interleukin-6, is an independent risk marker for subclinical carotid atherosclerosis. Stroke. 2004;35(7):1619-1624.

13. Huang G, Zhong XN, Zhong B, et al. Significance of white blood cell count and its subtypes in patients with acute coronary syndrome. Eur J Clin Invest. 2009;39(5):348-358.

14. Johnsen SH, Fosse E, Joakimsen O, et al. Monocyte count is a predictor of novel plaque formation: a 7-year follow-up study of 2610 persons without carotid plaque at baseline the Tromso Study. Stroke. 2005;36(4):715-719.

15. Madjid M, Awan I, Willerson JT, Casscells SW. Leukocyte count and coronary heart disease: implications for risk assessment. J Am Coll Cardiol. 2004;44(10):19451956.

16. Calin MV, Manduteanu I, Dragomir E, et al. Effect of depletion of monocytes/macrophages on early aortic valve lesion in experimental hyperlipidemia. Cell Tissue Res. 2009;336(2):237-248. 
17. Sunami $\mathrm{Y}$, Leithauser $\mathrm{F}$, Gul S, et al. Hepatic activation of IKK/NFkappaB signaling induces liver fibrosis via macrophage-mediated chronic inflammation. Hepatology. 2012;56(3):1117-1128.

18. van Rooijen N, van Kesteren-Hendrikx E. Clodronate liposomes: perspectives in research and therapeutics. $J$ Liposome Res. 2002;12(1-2):81-94.

19. Stoneman V, Braganza D, Figg N, et al. Monocyte/macrophage suppression in CD11b diphtheria toxin receptor transgenic mice differentially affects atherogenesis and established plaques. Circ Res. 2007;100(6):884-893.

20. Kinne RW, Brauer R, Stuhlmuller B, Palombo-Kinne E, Burmester GR. Macrophages in rheumatoid arthritis. Arthritis Res. 2000;2(3):189-202.

21. Smith JD, Trogan E, Ginsberg M, Grigaux C, Tian J, Miyata M. Decreased atherosclerosis in mice deficient in both macrophage colony-stimulating factor (op) and apolipoprotein E. Proc Natl Acad Sci U S A. 1995;92(18):8264-8268.

22. Martinet W, De Meyer GR. Selective depletion of macrophages in atherosclerotic plaques: myth, hype, or reality? Circ Res. 2007;100(6):751-753.

23. Danenberg HD, Fishbein I, Gao J, et al. Macrophage depletion by clodronatecontaining liposomes reduces neointimal formation after balloon injury in rats and rabbits. Circulation. 2002;106(5):599-605.

24. Burnett SH, Beus BJ, Avdiushko R, Qualls J, Kaplan AM, Cohen DA. Development of peritoneal adhesions in macrophage depleted mice. J Surg Res. 2006;131(2):296-301.

25. Grasset MF, Gobert-Gosse S, Mouchiroud G, Bourette RP. Macrophage differentiation of myeloid progenitor cells in response to M-CSF is regulated by the dual-specificity phosphatase DUSP5. J Leukoc Biol. 2010;87(1):127-135.

26. Di Gregoli K, Johnson JL. Role of colony-stimulating factors in atherosclerosis. Curr Opin Lipidol. 2012;23(5):412-421.

27. Wang Y, Mo X, Piper MG, et al. M-CSF induces monocyte survival by activating NF-kappaB p65 phosphorylation at Ser276 via protein kinase C. PLoS One. 2011;6(12):e28081.

28. de Bruin AM, Libregts SF, Valkhof M, Boon L, Touw IP, Nolte MA. IFNgamma induces monopoiesis and inhibits neutrophil development during inflammation. Blood. 2012;119(6):1543-1554.

29. Jaguin $\mathrm{M}$, Houlbert N, Fardel O, Lecureur V. Polarization profiles of human MCSF-generated macrophages and comparison of M1-markers in classically activated macrophages from GM-CSF and M-CSF origin. Cell Immunol. 2013;281(1):51-61.

30. Irvine KM, Andrews MR, Fernandez-Rojo MA, et al. Colony-stimulating factor1 (CSF-1) delivers a proatherogenic signal to human macrophages. J Leukoc Biol. 2009;85(2):278-288.

31. Zhang $\mathrm{HH}$, Basu S, Wu F, et al. Macrophage-colony stimulating factor is required for the production of neutrophil-promoting activity by mouse embryo fibroblasts deficient in G-CSF and GM-CSF. J Leukoc Biol. 2007;82(4):915-925.

32. Burnett SH, Kershen EJ, Zhang J, et al. Conditional macrophage ablation in transgenic mice expressing a Fas-based suicide gene. J Leukoc Biol. 2004;75(4):612623. 
33. von der Thusen JH, van Berkel TJ, Biessen EA. Induction of rapid atherogenesis by perivascular carotid collar placement in apolipoprotein E-deficient and low-density lipoprotein receptor-deficient mice. Circulation. 2001;103(8):1164-1170.

34. Kanters E, Pasparakis M, Gijbels MJ, et al. Inhibition of NF-kappaB activation in macrophages increases atherosclerosis in LDL receptor-deficient mice. J Clin Invest. 2003;112(8):1176-1185.

35. Legein B, Temmerman L, Biessen EA, Lutgens E. Inflammation and immune system interactions in atherosclerosis. Cell Mol Life Sci. 2013.

36. Ross R. Atherosclerosis--an inflammatory disease. $N$ Engl J Med. 1999;340(2):115-126.

37. Virmani R, Kolodgie FD, Burke AP, Farb A, Schwartz SM. Lessons from sudden coronary death: a comprehensive morphological classification scheme for atherosclerotic lesions. Arterioscler Thromb Vasc Biol. 2000;20(5):1262-1275.

38. Herembert T, Gogusev J, Zhu DL, Drueke TB, Marche P. Control of vascular smooth-muscle cell growth by macrophage-colony-stimulating factor. Biochem $\mathrm{J}$. 1997;325 ( Pt 1):123-128.

39. Inaba T, Yamada N, Gotoda T, et al. Expression of M-CSF receptor encoded by c-fms on smooth muscle cells derived from arteriosclerotic lesion. J Biol Chem. 1992;267(8):5693-5699.

40. Anderson KL, Smith KA, Perkin $\mathrm{H}$, et al. PU.1 and the granulocyte- and macrophage colony-stimulating factor receptors play distinct roles in late-stage myeloid cell differentiation. Blood. 1999;94(7):2310-2318.

41. DeKoter RP, Walsh JC, Singh H. PU.1 regulates both cytokine-dependent proliferation and differentiation of granulocyte/macrophage progenitors. EMBO J. 1998;17(15):4456-4468.

42. McKercher SR, Henkel GW, Maki RA. The transcription factor PU.1 does not regulate lineage commitment but has lineage-specific effects. J Leukoc Biol. 1999;66(5):727-732.

43. Wu Y, Madri J. Insights into monocyte-driven osteoclastogenesis and its link with hematopoiesis: regulatory roles of PECAM-1 (CD31) and SHP-1. Crit Rev Immunol. 2010;30(5):423-433.

44. Cecchini MG, Hofstetter W, Halasy J, Wetterwald A, Felix R. Role of CSF-1 in bone and bone marrow development. Mol Reprod Dev. 1997;46(1):75-83; discussion 83-74.

45. Winkler IG, Sims NA, Pettit AR, et al. Bone marrow macrophages maintain hematopoietic stem cell (HSC) niches and their depletion mobilizes HSCs. Blood. 2010;116(23):4815-4828.

46. Chow A, Lucas D, Hidalgo A, et al. Bone marrow CD169+ macrophages promote the retention of hematopoietic stem and progenitor cells in the mesenchymal stem cell niche. J Exp Med. 2011;208(2):261-271.

47. Kollet O, Dar A, Shivtiel S, et al. Osteoclasts degrade endosteal components and promote mobilization of hematopoietic progenitor cells. Nat Med. 2006;12(6):657664.

48. Cho KA, Joo SY, Han HS, Ryu KH, Woo SY. Osteoclast activation by receptor activator of NF-kappaB ligand enhances the mobilization of hematopoietic progenitor cells from the bone marrow in acute injury. Int J Mol Med. 2010;26(4):557-563. 
49. Horowitz MC, Bothwell AL, Hesslein DG, Pflugh DL, Schatz DG. B cells and osteoblast and osteoclast development. Immunol Rev. 2005;208:141-153.

50. Potteaux S, Gautier EL, Hutchison SB, et al. Suppressed monocyte recruitment drives macrophage removal from atherosclerotic plaques of Apoe-/- mice during disease regression. J Clin Invest. 2011;121(5):2025-2036.

51. Gautier EL, Huby T, Witztum JL, et al. Macrophage apoptosis exerts divergent effects on atherogenesis as a function of lesion stage. Circulation. 2009;119(13):17951804.

52. Tabas I. Macrophage death and defective inflammation resolution in atherosclerosis. Nat Rev Immunol. 2010;10(1):36-46.

53. Llodra J, Angeli V, Liu J, Trogan E, Fisher EA, Randolph GJ. Emigration of monocyte-derived cells from atherosclerotic lesions characterizes regressive, but not progressive, plaques. Proc Natl Acad Sci U S A. 2004;101(32):11779-11784.

54. Saha P, Geissmann F. Toward a functional characterization of blood monocytes. Immunol Cell Biol. 2011;89(1):2-4.

55. Robbins CS, Chudnovskiy A, Rauch PJ, et al. Extramedullary hematopoiesis generates $\mathrm{Ly}-6 \mathrm{C}(\mathrm{high})$ monocytes that infiltrate atherosclerotic lesions. Circulation. 2012;125(2):364-374.

56. Swirski FK, Nahrendorf M, Etzrodt M, et al. Identification of splenic reservoir monocytes and their deployment to inflammatory sites. Science. 2009;325(5940):612616. 



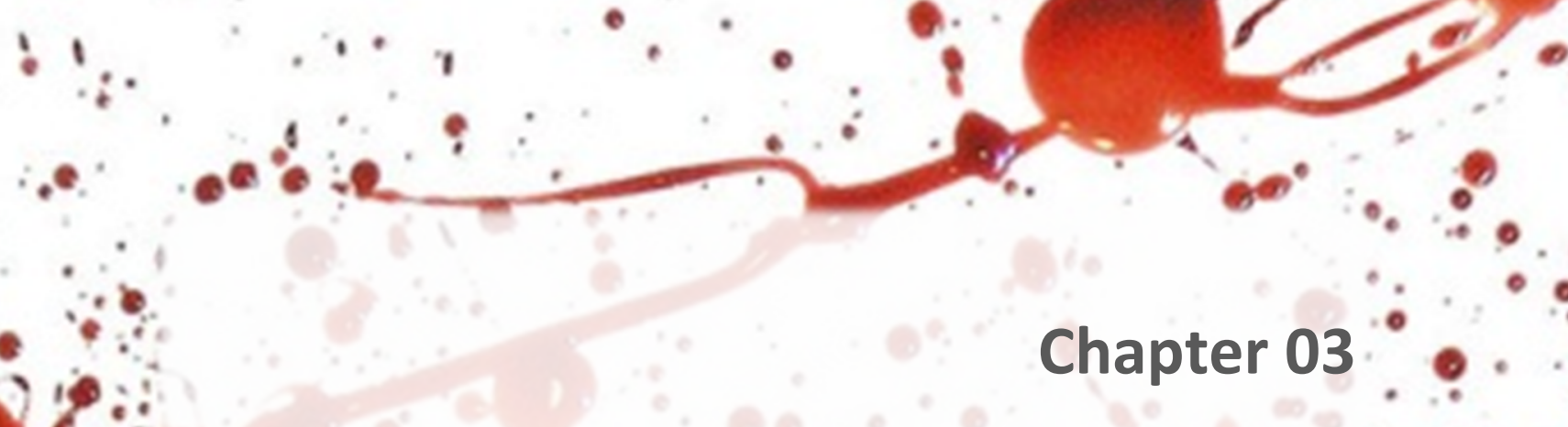

\section{Hematopoietic G-protein-coupled receptor kinase 2 deficiency ${ }^{8} \cdot \therefore$} decreases atherosclerotic lesion 1 formation in LDL receptor- 60
knockout mice

Jeroen JT Otten*, Saskia C de Jager*, Annemieke Kavelaars, Tom Seijkens, e Ilze Bot, Erwin Wijnands, Linda Beckers, Marijke M Westra, Martine Bot, Matthias Busch, Beatriz Bermudez, Theo van Berkel TJ, Cobi J Heijnen, Erik AL Biessen

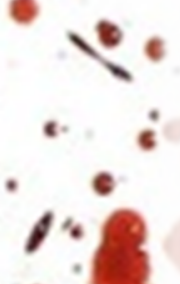
3. $x=$

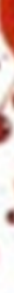




\begin{abstract}
Leukocyte chemotaxis is deemed instrumental in initiation and progression of atherosclerosis. It is mediated by G-protein-coupled receptors (e.g., CCR2 and CCR5), the activity of which is controlled by G-protein-coupled receptor kinases (GRKs). In this study, we analyzed the effect of hematopoietic deficiency of a potent regulator kinase of chemotaxis (GRK2) on atherogenesis. LDL receptordeficient $\left(L D L r^{-1}\right)$ mice with heterozygous hematopoietic GRK2 deficiency, generated by bone marrow transplantation $(n=15)$, displayed a dramatic attenuation of plaque development, with $79 \%$ reduction in necrotic core and increased macrophage content. Circulating monocytes decreased and granulocytes increased in $G R K 2^{--}$chimeras, which could be attributed to diminished granulocyte colony-forming units in bone marrow. Collectively, these data pointed to myeloid cells as major mediators of the impaired atherogenic response in $G R K 2^{--}$chimeras. $L D L r^{-/}$mice with macrophage/granulocyte-specific GRK2 deficiency (LysM-Cre GRK2 $2^{f l o x / f l o x} ; n=8$ ) failed to mimic the aforementioned phenotype, acquitting these cells as major responsible subsets for GRK2 deficiency-associated atheroprotection. To conclude, even partial hematopoietic GRK2 deficiency prevents atherosclerotic lesion progression beyond the fatty streak stage, identifying hematopoietic GRK2 as a potential target for intervention in atherosclerosis.
\end{abstract}




\section{Introduction}

Leukocyte migration toward atherosclerotic lesions is deemed instrumental in atherosclerosis initiation and progression ${ }^{1}$. By releasing cytokines ${ }^{2}$ and proteolytic enzymes, infiltrated leukocytes may also promote plaque destabilization, a process generally preceding thrombotic rupture and subsequent acute cardiovascular events. Because leukocyte migration may be a potentially attractive target for the prevention or control of plaque destabilization and ischemic disease, this research focuses on the pivotal role of leukocyte migration in atherosclerosis.

Chemokines, a family of cytokines with strong chemotactic capacity ${ }^{3,4}$, are key regulators of leukocyte transmigration into the vessel wall during atherogenesis ${ }^{5,6}$. Chemokines are inducible and control cellular recruitment, especially to inflammatory sites ${ }^{7}$. In response to inflammatory stimuli, chemokines can be released by various cell types relevant to atherosclerosis, including endothelial cells, platelets, and leukocytes ${ }^{8-11}$. They act by binding to dedicated receptors of the G-protein-coupled receptor (GPCR) family. GPCRs are coupled to heterotrimeric $G$ proteins and induce, after ligand binding, activation of downstream signaling cascades ${ }^{12,13}$.

The expression and activity of GPCRs is regulated not only at the mRNA or protein level but also functionally. One such mechanism is receptor desensitization, which dampens the response to prolonged or repeated stimuli 14,15. Dedicated GPCR kinases (GRKs) can induce receptor desensitization by phosphorylation of the ligand-occupied receptor, thereby enhancing its affinity for arrestin proteins. Binding of arrestins to the phosphorylated receptor will result in uncoupling and internalization of the receptor ${ }^{16,17}$. The GRK family comprises 7 serine/threonine kinases ${ }^{18,19}$. They regulate a range of processes, including cell migration ${ }^{20,21}$, neuronal pathways ${ }^{22}$, and cell survival ${ }^{23}$. Dysfunction of GRKs, particularly GRK2, has been implicated in various human pathologies, such as multiple sclerosis ${ }^{24}$, rheumatoid arthritis ${ }^{25}$, chronic pain ${ }^{26}$, and heart failure ${ }^{27}$, whereas GRK2 overexpression induces hypertension 28,29. Recently, GRK5 was shown to be involved in atherogenesis in mice through smooth muscle cells and endothelial cells. However, monocyte migration and function were also affected by GRK5 deficiency ${ }^{30}$. Because key chemokine receptors in atherosclerosis [CC-motif chemokine receptor (CCR) 2 and CCR5] are targeted by GRK2 ${ }^{\text {ref }}{ }^{31}$, decreased levels of this receptor kinase conceivably result in excessive migration of inflammatory cells toward 
atherosclerotic lesions. Therefore, the aim of this study was to assess the role of leukocyte specific GRK2 deficiency on the development of atherosclerosis in LDL receptor (LDLr)-deficient mice.

\section{Materials \& Methods}

\section{Animals}

Female $L D L r^{-/}$mice ${ }^{32}$ were obtained from the animal breeding facility at Maastricht University (Maastricht, The Netherlands). Mice were fed a regular chow diet (RM3; Special Diet Services, Essex, UK) or a Western-type diet (WTD; 0.25\% cholesterol; Special Diet Services). Drinking water and food were provided ad libitum. Experiments were performed at the Maastricht University animal facility. Male heterozygous GRK2 $\left(\right.$ GRK2 $\left.{ }^{-/}\right)$mice ${ }^{33}$, LysM-Cre GRK2 $2^{\text {flox/flox }}$ mice ${ }^{34,35}$, and littermate controls were used as donors for bone marrow transplantations and in vitro assays. Donor mice were maintained at the Utrecht University Medical Center (Utrecht, The Netherlands) animal facilities. All experimental protocols were approved by the Maastricht University ethics committee for animal experiments.

\section{Bone Marrow Transplantation}

Female $L D L r^{-1}$ mice were lethally irradiated with a single dose (9 Gy, 0.5 Gy/min, MU 15 F/225 kV; Philips Healthcare, Best, The Netherlands) total-body irradiation 1 day before transplantation. On the next day, irradiated recipients received an intravenous tail vein injection of $5 \times 10^{6}$ bone marrow cells. Bone marrow was isolated from $G R K 2^{-/}$and littermate controls or LysM-Cre $G R K 2^{f l o x}$ flox and littermate controls by flushing femurs and tibiae. Drinking water supplemented with antibiotics $[60 \mathrm{U} / \mathrm{mL}$ polymyxin B sulfate (SigmaAldrich, St. Louis, MO, USA) and $100 \mathrm{~g} / \mathrm{mL}$ neomycin (Sigma-Aldrich)] was provided starting 1 week before the bone marrow transplantation. After 6 weeks of recovery, mice were fed a WTD for 12 weeks and subsequently euthanized.

\section{In vivo Migration and Proliferation}

Female wild-type (WT) controls and $\mathrm{GRK}^{-/}$chimeras $(\mathrm{n}=4 / 5)$ were intraperitoneally injected 24 hours before euthanasia with PBS or CC-motif chemokine ligand (CCL) 5 [RANTES; 200 ng/mouse; Peprotech, London, UK] to 
stimulate cell migration. At euthanasia, leukocytes were isolated by peritoneal lavage (sterile PBS), and lavages were subsequently analyzed for leukocyte content on an automated differential cell counter (XT-2000i; Sysmex, Norderstedt, Germany).

Splenocytes were isolated from spleen, after the tissue was gently homogenized over a $70 \mu \mathrm{m}$ cell strainer (BD Biosciences, San Diego, CA, USA). To remove erythrocytes, cell suspensions were incubated in ice-cold erythrocyte lysis buffer $\left(155 \mathrm{mM} \mathrm{NH}_{4} \mathrm{Cl}\right.$ in $10 \mathrm{mM}$ Tris/ $\mathrm{HCl}, \mathrm{pH}$ 7.2) for $3 \mathrm{~min}$. Splenocytes ( $n=6 /$ group) were then resuspended in RPMI 1640 medium (Invitrogen, Paisley, UK) supplemented with I-glutamine, $100 \mathrm{U} / \mathrm{mL}$ streptomycin/penicillin, and 10\% FCS and cultured for $48 \mathrm{~h}$ in quadruplicate in a 96-well round-bottom plate ( $2 \times 10^{5}$ cells/well; Corning, Lowell, MA, USA). Cells were stimulated with CCL5 (RANTES; $100 \mathrm{ng} / \mathrm{mL}$ ). Concanavalin A (ConA; $8 \mathrm{~g} / \mathrm{mL}$; Sigma-Aldrich) was used as a positive control. Proliferation was determined after addition of $\left.{ }_{3} \mathrm{H}\right]$ thymidine $(0.5 \mathrm{Ci} /$ well; Amersham Biosciences, Roosendaal, The Netherlands), incubation of the cells for the last $16 \mathrm{~h}$, and measurement of cell-associated (incorporated) $\left.{ }_{3} \mathrm{H}\right]$ thymidine by a liquid scintillation analyzer (Tri-Carb 2900R; PerkinElmer, Waltham, MA, USA).

\section{Histological Analysis}

For the GRK2\% study, aortic roots were isolated, and $10-\mu \mathrm{m}$ cryostat sections were stained with Oil-Red-O. Total plaque area and necrotic core size were determined in 7 sections/animal. Corresponding sections on separate slides were stained immunohistochemically with a macrophage-specific antibody (MOMA-2; Serotec, Oxford, UK). For the LysM-Cre GRK2 $2^{f l o x / f l o x}$ study, aortic root sections were collected, formalin-fixed, and paraffin-embedded. Subsequently, $4 \mu \mathrm{m}$-thick sections were stained with hematoxylin and eosin. Lesion size and necrotic core area were determined in 3-7 sections/animal. Corresponding sections on separate slides were stained immunohistochemically with a macrophage-specific antibody (MAC-3; BD, Franklin Lakes, NJ, USA). Movat's pentachrome stain was used to elastin and to obtain insight into plaque progression stage and stability. Plaque collagen content was quantified based on Sirius Red staining and subsequent analysis of the plaques under polarized light. Vascular smooth muscle cells were analyzed more in depth by $\alpha$-smooth muscle cell-specific actin ( $\alpha$ SMA; Sigma-Aldrich) staining. T-lymphocyte infiltration was quantified based on staining with a $\operatorname{CD} 3 \varepsilon$-specific antibody 
(CD3ع; Dako, Glostrup, Denmark). Apoptosis was visualized using a TUNEL kit (Roche, Woerden, The Netherlands). Apoptotic cell content was determined by assessment of the TUNEL-positive area per section. Histological analyses were performed in a blinded manner by an independent operator using Quantimet (Leica, Wetzler, Germany) with QWin3 quantification software (Leica).

\section{In vitro Macrophage Culture}

Bone marrow cells were isolated from WT and $G R K 2^{-/}$mice, and cells were pooled and cultured in RPMI 1640 medium supplemented with fetal calf serum and L929 conditioned medium to generate bone marrow-derived macrophages (BMDMs) as described previously ${ }^{36}$. Medium was replaced every 3 days, and differentiated BMDMs were used for in vitro assays after 7 days.

\section{Macrophage Foam Cell Formation Assay}

BMDMs were cultured and plated in 24-well cell cluster plates (Corning). Macrophages were incubated for 24 hours with VLDL $(25 \mu \mathrm{g} / \mathrm{mL})$ to induce foam cell formation. Subsequently, Oil Red $O$ staining was performed to visualize the cellular lipid accumulation. Pictures were taken using a DM IL LED fluorescence microscope (Leica) with QWin3 software, and foam cell formation was scored in a blinded manner by an independent operator, calculating the number of positive Oil Red O-stained cells, as well as the staining intensity at a per cell basis in $>3$ fields/condition ( $\times 100$ view).

\section{Macrophage Apoptosis and Phagocytosis Assay}

BMDMs were cultured and plated in 48-well cell cluster plates (Corning). Macrophages were incubated for 18 hours in plain medium or medium supplemented with camptothecin (CPT; $500 \mu \mathrm{M}$ ) or oxidized LDL (oxLDL; 50 $\mu \mathrm{g} / \mathrm{mL}$ ). oxLDL was produced as described previously ${ }^{37}$. Cells were labeled with annexin V-Alexa 488 (Invitrogen) and stained with propidium iodide to detect apoptosis and necrosis, respectively. Pictures were taken using a DM IL LED fluorescence microscope with Qwin3 software. Overlays were analyzed with ImageJ software (U.S. National Institutes of Health, Bethesda, MD, USA) to quantify the number of apoptotic and necrotic cells per field.

To investigate phagocytic capacity, cultured BMDMs were labeled with Cell Tracker Red (Invitrogen). Jurkat T cells labeled with Cell Tracker Green (Invitrogen) were exposed to a UV light source (UVS-26, 6-W bulb, 0.02 $\mathrm{J} / \mathrm{s} / \mathrm{cm}^{2}$; UVP, Upland, CA, USA) for 20 minutes and incubated $\left(37^{\circ} \mathrm{C}\right.$ with $5 \%$ 
$\mathrm{CO}_{2}$ ) for 45 minutes to induce apoptosis. Apoptotic Jurkat cells were added to the BMDMs at a ratio of 5:1 and incubated for 50 minutes. Macrophages were fixed with $1 \%$ paraformaldehyde and counterstained with DAPI (Invitrogen). Pictures were taken (DM IL LED fluorescence microscope), and overlays were analyzed with ImageJ.

\section{Flow Cytometry}

At euthanasia, blood, spleen, and peritoneal leukocytes were collected. Blood and peritoneal leukocytes were analyzed for cellular composition on an automated differential cell counter (XT-2000i). Single-cell suspensions were made from spleen by crushing the tissue over a 70- $\mu \mathrm{m}$ cell strainer (BD) after treatment with DNase and Liberase (Roche). Erythrocytes in blood and spleen were removed by incubation with hypotonic lysis buffer $\left(8.4 \mathrm{~g}\right.$ of $\mathrm{NH}_{4} \mathrm{Cl}$ and $0.84 \mathrm{~g}$ of $\mathrm{NaHCO}_{3}$ per liter of distilled water). Nonspecific Fc receptor binding was blocked by the addition of anti-CD16/CD32 antibody (eBioscience, San Diego, CA, USA). To determine lymphocyte subsets, cells were labeled with CD3E-FITC, CD8 $\alpha$-eFluor450, B220-PE-Cy7, CD25-APC (all eBioscience), and $\mathrm{CD} 4-P e r C P(B D)$. To detect monocytes and granulocytes, the cells were labeled with CD11b-PE-Cy7, Ly6G-PE (BD), and Ly6C-FITC (Miltenyi Biotec, Bergisch Gladbach, Germany). Dendritic cells were detected by labeling with CD3عPerCPCy5.5, CD19-PerCP-Cy5.5, MHC II-FITC, CD8 $\alpha$-eFluor450 (all eBioscience), CD11C-PE-Cy7, and CD4-APC-H7 (both BD) for resident dendritic cells. Chemokine receptor expression was determined by labeling peripheral blood leukocytes with CCR5-PE, CCR7-PerCP-Cy5.5, CD3e-eFluor450, NK1.1-APC (all eBioscience), CD11b-PE-Cy7, Ly6G-APC-Cy7, B220-V500 (all BD), and Ly6C-FITC (Miltenyi). After labeling, the samples were washed and analyzed on a FACSCanto II flow cytometer (BD). Samples and buffers were kept on ice throughout the experiment. Gating strategies used for the different flow cytometry stainings are shown in the supplemental data. 


\section{Colony-Forming Unit (CFU) Assay}

Bone marrow cells were isolated from one tibia and one femur per mouse. WT,

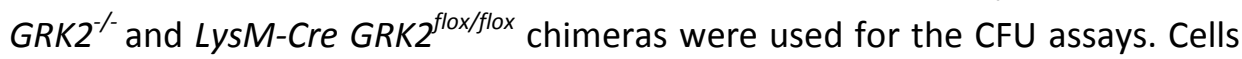
were counted twice using a count chamber, and the concentration was calculated for each sample. A total of 10,000 bone marrow cells/well were added to $2 \mathrm{~mL}$ of methylcellulose medium with recombinant cytokines (MethoCult Medium; StemCell Technologies, Grenoble, France). After incubation for 7 days $\left(37^{\circ} \mathrm{C}\right.$ with $\left.5 \% \mathrm{CO}_{2}\right)$, the total number of colonies was quantified by an independent operator, and granulocyte-macrophage CFU (GM-CFU), granulocyte CFU (G-CFU), and macrophage CFU (M-CFU) colonies were specified based on morphology of the individual cells and the colony as a whole.

\section{Statistical Analysis}

Data are expressed as mean \pm sem. To compare individual groups, a 2-tailed Student's $t$ test was used; nonparametric data were analyzed using a MannWhitney $U$ test. All analyses were performed using GraphPad Prism 5 software (GraphPad Software Inc., LA Jolla, CA, USA), and values of $P<0.05$ were considered statistically significant.

\section{Results}

\section{Partial Leukocyte GRK2 Deficiency Attenuates Atherosclerosis}

Partial hematopoietic GRK2 deficiency did not affect body weight and total cholesterol levels (Supplemental Fig. S1). Hematopoietic GRK2 ${ }^{-}$chimeras in $L D L r^{-1}$ mice $(n=15)$ showed significantly decreased atherosclerotic lesion development in the aortic root compared with that of controls $(403.0 \pm 43.8 \mathrm{x}$ $10^{3}$ vs. $585.0 \pm 56.4 \times 10^{3} \mu \mathrm{m}^{2}, P=0.017$; Fig. $1 A$ ). The necrotic core size was 4 fold smaller in heterozygous GRK2 than in WT chimeras ( $9.5 \pm 2.3$ vs. $44.6 \pm$ $6.1 \%, P<0.0001$; Fig. 1B). The TUNEL-positive apoptotic cell burden (Fig. 1C) did not differ between GRK2 and WT chimeras. Further differentiation for TUNELpositive cell density in the cap vs. the atheroma revealed that in both WT and GRK $^{-}$chimeras $95 \%$ of TUNEL-positive cells were located in the atheroma (data not shown). Moreover, because we did not observe a shift in localization over these two compartments and because the atheroma mainly consists of macrophages (next to cell debris), we may infer that partial GRK2 deficiency 
has not affected macrophage apoptosis. Next, collagen content was not affected in $\mathrm{GRK}^{-/}$chimeras compared with WT chimeras (Fig. 1D).

Surprisingly, intimal MOMA-2-positive macrophage content in heterozygous GRK2 chimeras was 2-fold higher than that of controls ( $32.3 \pm 2.9$ vs. $15.3 \pm 2.4 \%, P=0.00015$; Fig. $1 E$ ). Analysis of the lesions did not reveal significant differences in intimal (CD3E+) lymphocyte numbers (data not shown). Intimal smooth muscle cell content, as assessed by smooth muscle cell-specific actin staining, was increased by heterozygous GRK2 deficiency (Fig. 1F). General assessment of plaque morphology, from Movat's pentachrome staining, showed a significant reduction of overall necrosis in lesions from $\mathrm{GRK}^{-/}$chimeras (Supplemental Fig. S2A). In line with the results obtained based on MOMA-2 staining, foam cell macrophage content was significantly increased in GRK2 ${ }^{-/}$lesions (Supplemental Fig. S2B). For neutrophil infiltration, it was observed that the number of granulocytes per lesion were very low, which was confirmed by the virtual absence of positive Ly6G staining (data not shown), whereas intraplaque/adventitial neutrophil numbers tended to be decreased in heterozygous GRK2 animals, based on morphological scoring of the lesions (Supplemental Fig. S2C). We did, however, observe a significant decrease in the amount of cells adhering to the luminal side of the atherosclerotic lesions in the GRK2\% chimeras (Supplemental Fig. S2D), which could not be further attributed to a specific cell type. Overall, atherosclerotic lesions in $\mathrm{GRK}^{-/-}$chimera were considered less advanced and more stable than those of controls, despite unchanged collagen levels (Supplemental Fig. S2E), based on plaque morphology and composition. 
A

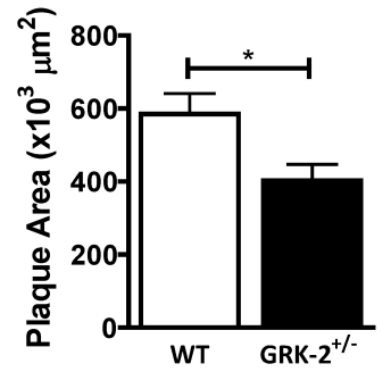

D

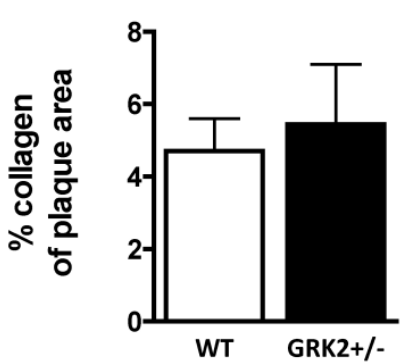

B

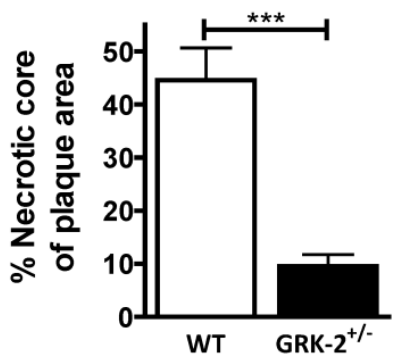

$\mathbf{E}$

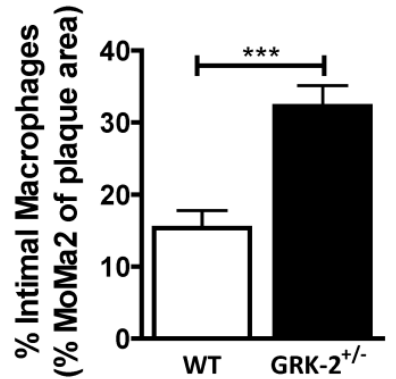

C

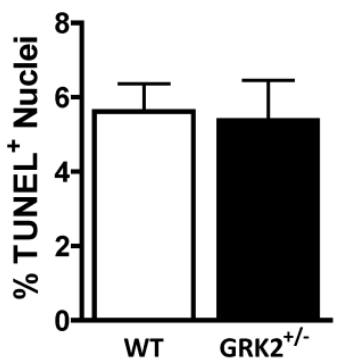

$\mathbf{F}$

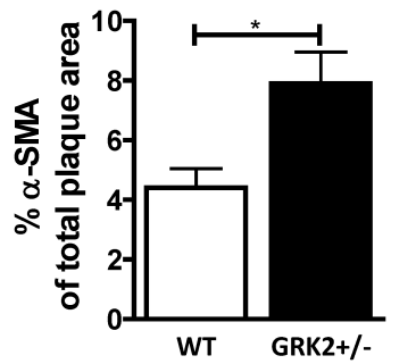

G

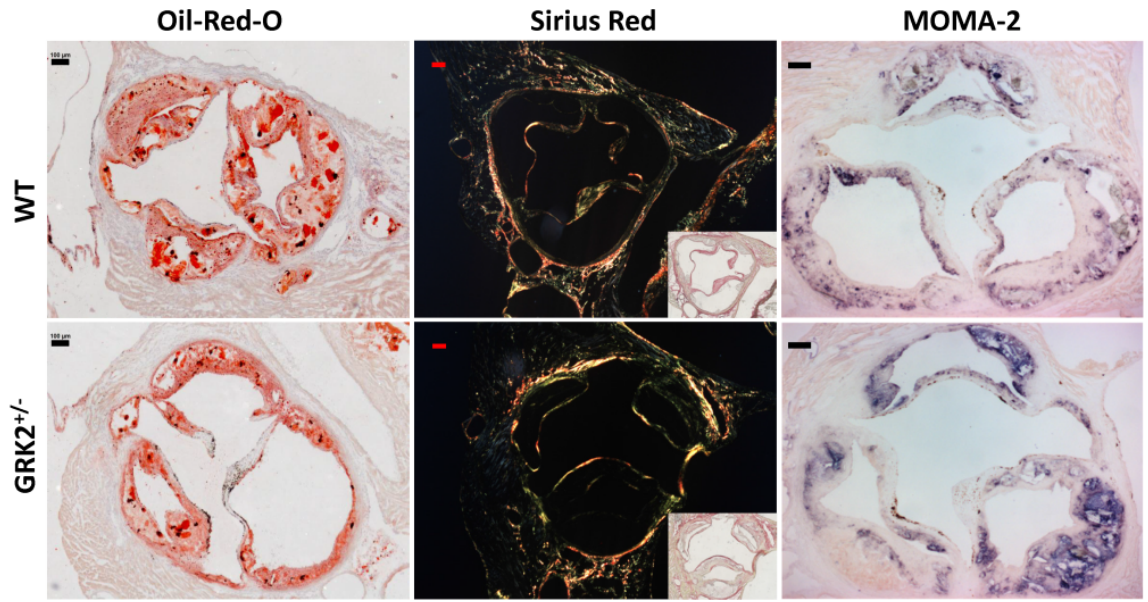

Figure 1 - Atherosclerotic plaque development is attenuated in $\mathrm{GRK2}^{+/-}$chimeras.

(A) Plaque area and (B) necrotic core size were determined based on Oil-Red-O staining. (C) Relative number of apoptotic cells was determined by TUNEL staining. (D) Collagen content was measured by analysis of Sirius Red staining under polarized light. (E) Macrophage content was measured in sections stained for the monocyte/macrophage marker MOMA-2. (F) Smooth muscle cell content was measured by an staining against alpha-smooth muscle cell actin ( $\alpha S M A)$. (G) Representative pictures of Oil-Red-O, Sirius Red (polarized light; insert bright field image), and MoMa-2 staining are shown for wild type and $\mathrm{GRK}^{+/-}$chimeras. The top panels represent WT and the bottom panels GRK2 chimeras. Wild type controls (WT) are shown in white and $\mathrm{GRK}^{+/-}$chimeras are shown in black; scale bars are $100 \mu \mathrm{m} .{ }^{*} p<0.05$ and ${ }^{* * *} p<0.001$ 
A

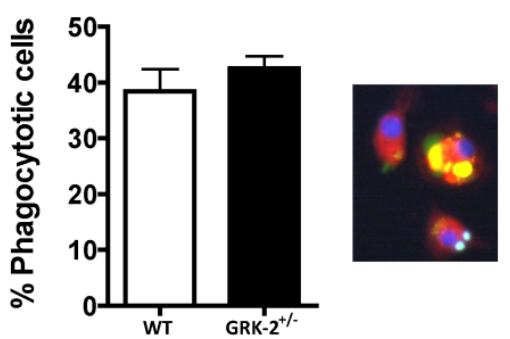

C

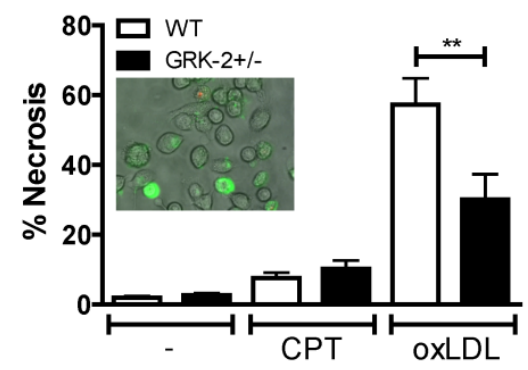

B

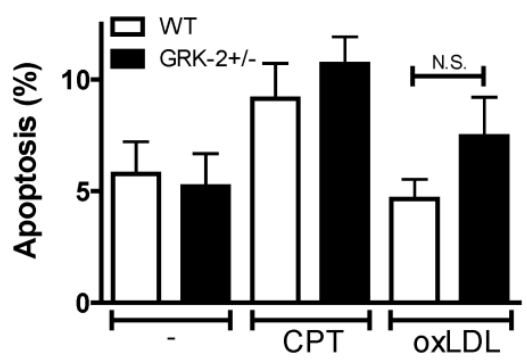

D

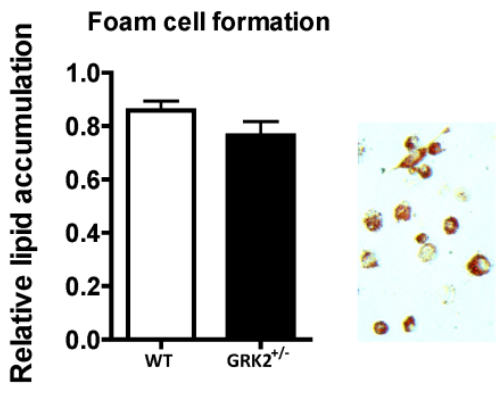

Figure 2 - Phagocytosis, apoptosis, and foam cell formation of bone marrow derived macrophages is not affected in vitro.

(A) The phagocytic capacity of BMDM for apoptotic Jurkat T-cells was determined in vitro. Phagocytosis was determined by scoring yellow staining in overlays from macrophages (cell tracker red), apoptotic Jurkat T-cells (cell tracker green) and nuclei (DAPI; blue). (B) In vitro apoptosis and (C) necrosis of bone marrow-derived macrophages was measured at baseline (-) and upon camptothecin (CPT), or oxidized LDL (oxLDL) exposure. Cell death was quantified based on Annexin V-Alexa488 (apoptosis; green) and propidium iodide staining (necrosis; red/orange). (D) In vitro foam cell formation was measured upon VLDL exposure for 24 hours using Oil-Red-O staining (red; $400 \times$ magnification). White bars represent wild type controls (WT) and black bars $\mathrm{GRK}^{+/-}$chimeras $\left(\mathrm{GRK}^{+/-}\right)$. ${ }^{* *} p<0.01$.

Macrophage GRK2 Deficiency does not affect Phagocytosis, Apoptosis, or Foam Cell Formation

The major differences in plaque composition, regarding necrotic core and intimal macrophage content, prompted us to study the effect of GRK2 deficiency on macrophage function. Reduced GRK2 expression did not affect phagocytosis of apoptotic Jurkat $\mathrm{T}$ cells by BMDMs (Fig. 2A), rendering it unlikely that the reduced necrotic core size is attributable to increased phagocytosis.

In line with the unchanged $\mathrm{TUNEL}^{+}$cell density observed in plaques from WT and GRK2 ${ }^{-\%}$ chimeras, in vitro we observed no effects of GRK2 
heterozygosity $(n=9)$ on the susceptibility for apoptosis of BMDMs at baseline or in the presence of CPT (Fig. 2B), nor did we observe differences in necrosis between BMDMs at baseline and after camptothecin (CPT) treatment (Fig. 2C). Notably, oxLDL-induced necrosis was markedly attenuated in GRK2 $\%$ compared with WT BMDMs (30.1 \pm 7.2 vs. $57.2 \pm 7.6 \%, P=0.019$; Fig. $2 C)$. Thus, partial GRK2 deficiency was seen to reduce oxLDL-induced necrosis, which could have contributed to the phenotypic differences in necrotic core size in vivo.

A

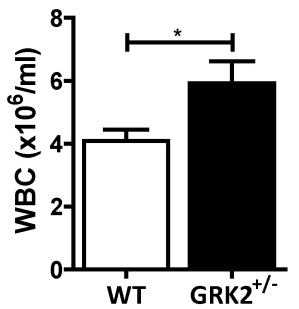

D

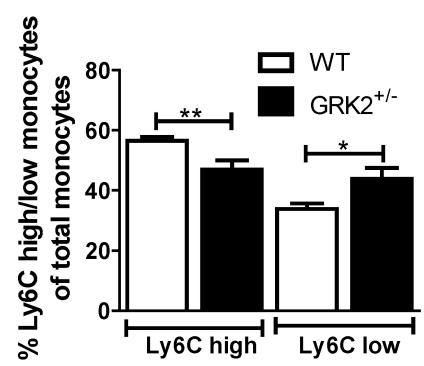

G

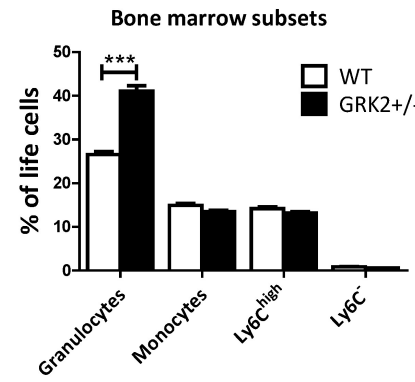

B

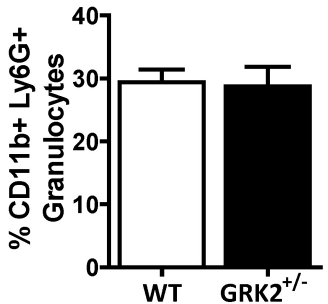

E

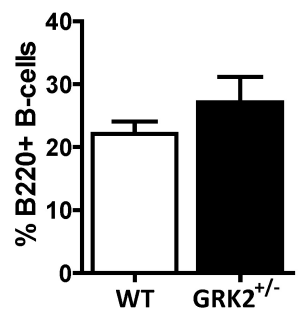

C

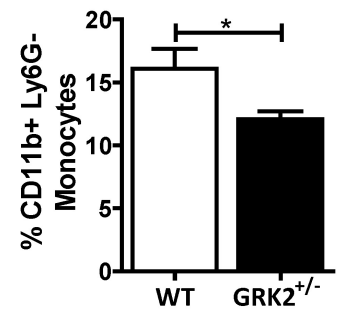

$\mathbf{F}$

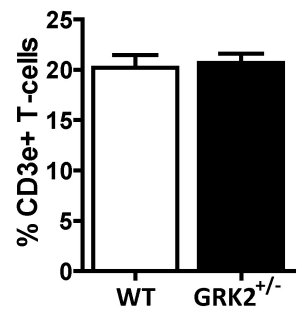

Blood
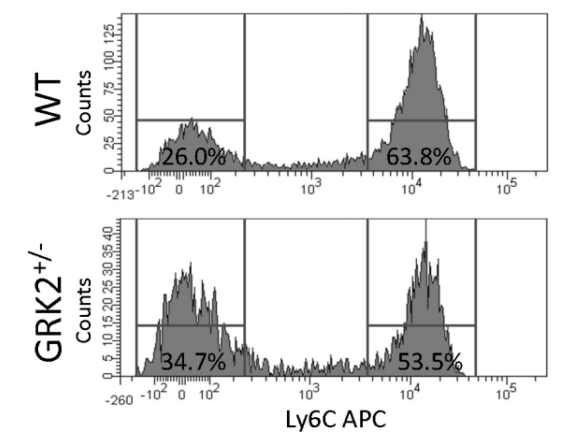

Figure 3 - GRK2 ${ }^{+/-}$chimeras have perturbed leukocyte patterns in circulation.

(A) Absolute number of white blood cells (WBC) were measured using a Sysmex differential cell counter. The relative fractions of leukocyte subsets were measured using flow cytometry analysis on a FACS Canto II (BD). (B) Granulocytes, (C) monocytes, (D) monocyte Ly6C subsets, (E) B-cells, and (F) T-cells were measured in the circulation of WT control (white bars) and $\mathrm{GRK}^{+/-}$chimeras (black bars). (G) Myeloid subsets were measured in bone marrow of donor mice. $(H)$ Representative histograms of Ly $6 C^{\text {high }}$ and Ly6C $\mathrm{C}^{\text {low }}$ distribution in blood of WT and $\mathrm{GRK2}^{+/-}$chimeras. ${ }^{*} p<0.05$ and ${ }^{* *} p<0.01$. 
Because macrophage and foam cell macrophage content was significantly increased in the $\mathrm{GRK}^{+/-}$lesions compared with the WT lesions, we investigated in vitro whether GRK2 heterozygosity by itself influences foam cell formation. However, we were unable to detect any effects on the number of macrophage-derived foam cells nor on the level of lipid accumulation per cell after VLDL incubation (Fig. 2D), indicating that the observed plaque foam cell effects are not caused by GRK2 deficiency-associated changes in macrophage lipid handling.

\section{Circulating Leukocytes are affected by GRK2 Deficiency}

Peripheral blood analysis revealed increased circulating leukocyte numbers in GRK2 $2^{-/-}$chimeras (5.9 $\pm 0.6 \times 10^{6}$ vs. $4.1 \pm 0.4 \times 10^{6}$ cells $/ \mathrm{mL}, P=0.03$; Fig. $\left.3 A\right)$. Indepth analysis of peripheral blood samples from WT and $G R K 2^{-/-}$chimeras fed a WTD $(0.25 \%$ cholesterol) by flow cytometry indicated that there was no relative difference in granulocyte $\left(\mathrm{CD} 11 \mathrm{~b}^{+} / \mathrm{Ly}_{6 \mathrm{G}}{ }^{+}\right)$abundance (Fig. 3B). Circulating monocyte $\left(\mathrm{CD} 11 \mathrm{~b}^{+} / \mathrm{Ly}_{6 \mathrm{G}}\right)$ ) numbers were significantly lowered in GRK $2^{\%}$ compared with those in WT chimeras (12.09 \pm 0.61 vs. $16.09 \pm 1.58 \%$ monocytes, $P=0.039$; Fig. $3 C$ ) and displayed a decreased Ly $6 C^{\text {high }} /$ Ly $6 C^{\text {low }}$ ratio (Fig. 3D). No genotype-related differences were observed in B cells (B220+; Fig. $3 E)$ and $\mathrm{T}$ cells (CD3 $\varepsilon^{+}$; Fig. $\left.3 F\right)$ or in T-cell subsets (CD4 ${ }^{+} / \mathrm{CD}^{+}$; data not shown). Spleen resident dendritic cell content $\left(\mathrm{CD} 11 \mathrm{c}^{+}\right)$and resident dendritic cell composition ( $\mathrm{CD}^{+} / \mathrm{CD}^{+} /$double-negative; data not shown) did not reveal any differences between WT and GRK2\%- chimeras. In spleen, we did not observe any significant differences other than a relative decrease in the number of granulocytes in the $G R K 2^{-/-}$chimeras compared with that in WT controls $(3.30 \pm$ 0.32 vs. $2.15 \pm 0.21 \%$ granulocytes; Supplemental Fig. S3A). B cells and T cells did not show any differences in spleen (Supplemental Fig. S3B, C), while splenic monocytes, and in particular the Ly6 $C^{\text {high }}$ subset, showed a tendency toward reduced numbers, as observed in peripheral blood (Supplemental Fig. S3D, E). In bone marrow, on the other hand, we observed no differences in monocytes or monocyte subsets (Fig. 3G). This result also indicates that impaired egress might be underlying the observed monocytopenia. Granulocytes are accumulating in the bone marrow of GRK2-deficient mice (Fig. 3G), but this does not translate into any effects on the atherosclerotic lesion. In short, these findings indicate that the effects of GRK2 deficiency on plaque formation and on circulating leukocytes mainly affect the myeloid lineage, monocytes and granulocytes, in blood, spleen, and bone marrow. 
A

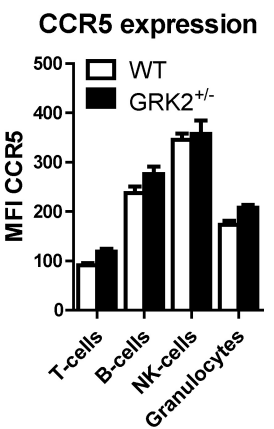

B

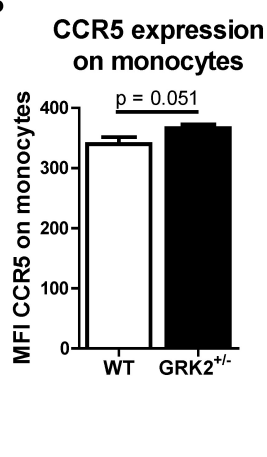

C

CCR5 expression on monocytes

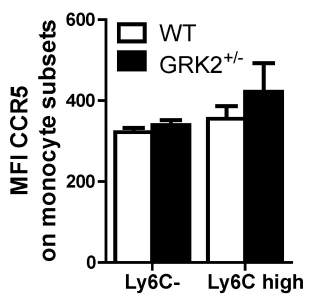

D

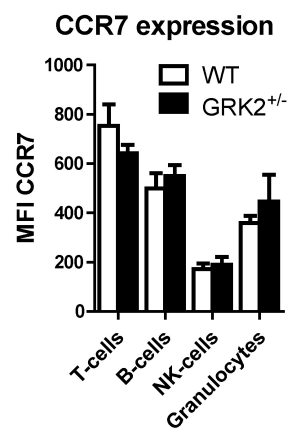

Figure 4 - Chemokine receptor expression on leukocytes from WT and $\mathrm{GRK2}^{+/-}$chimeras. Chemokine receptor (CCR5 and CCR7) expression was measured on circulating leukocytes from WT control (white bars) and GRK2 ${ }^{+-}$chimeras (black bars). (A) CCR5 expression on T-cells, Bcells, NK-cells, granulocytes, and (B) monocytes. (C) Ly6C monocyte subsets showed differential CCR5 expression. (D) CCR7 expression on T-cells, B-cells, NK-cells, and granulocytes.

The reported role of GRK2 in chemokine receptor desensitization led us to investigate effects of $G R K 2^{-1-}$ deficiency on leukocyte expression of relevant chemokine receptors such as CCR5 and CCR7 by flow cytometry. No statistically significant increase in CCR5 expression could be observed in any of the leukocyte subsets at baseline (Fig. 4A). However, CCL5-stimulated monocytes and, in particular, the proinflammatory Ly6 $\mathrm{C}^{\text {high }}$ subset, showed borderline significance toward increased CCR5 expression in GRK2 $2^{--}$chimeras compared with WT controls ( $P=0.051$; Fig. $4 B, C$ ). For CCR7 we could not detect any significant differences in expression for any leukocyte subset tested (Fig. $4 D)$.

Baseline Proliferation and CCL5-mediated Migration are increased in $\mathrm{GRK}^{-/-}$Animals

Proliferation was measured by $\left[{ }^{3} \mathrm{H}\right]$ thymidine incorporation in total splenocytes under baseline conditions (PBS) or after stimulation for $16 \mathrm{~h}$ with CCL5 (RANTES) or with the general mitogen ConA. It was observed that baseline proliferation (control) was increased in $G R K 2^{-/}$splenocytes compared with that in WT controls $(P<0.05$; Fig. $5 A)$. However, the proliferation index in CCL5 or ConA stimulated cells, corrected for baseline proliferation, was unaffected in GRK $^{\%}$ splenocytes (Fig. 5B). 

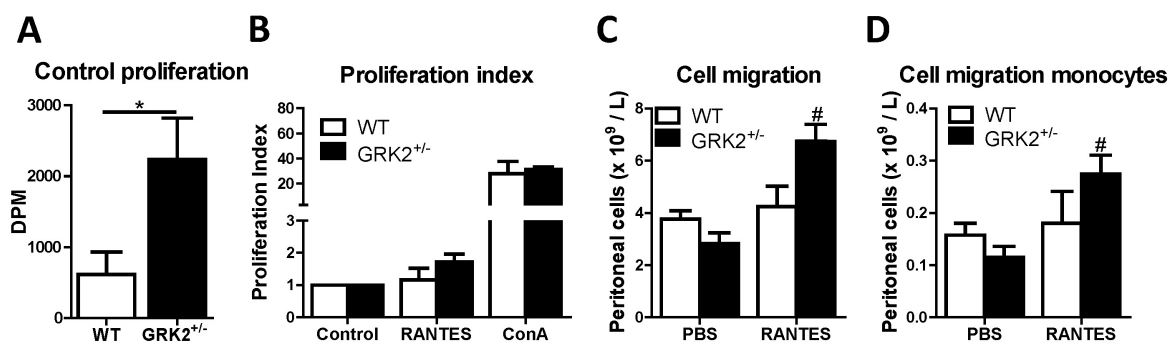

Figure 5 - Baseline proliferation and migration upon CCL5 stimulation is increased in $\mathrm{GRK}^{+/}$ chimeras

(A) Baseline proliferation (unstimulated) was determined in WT and $\mathrm{GRK}^{+/-}$splenocytes. (B) Proliferation index (corrected proliferation for control condition) was determined for CCL5 (100 $\mathrm{ng} / \mathrm{mL}$; RANTES) or Concanavalin A (100 ng/mL; ConA) stimulation. (C) Total cell migration and (D) monocyte migration in WT control and $\mathrm{GRK}^{+/-}$chimeras upon i.p. CCL5 (200 ng; RANTES) challenge. ${ }^{*} p<0.05$ and $\# p<0.05$ compared to GRK2 ${ }^{+/-}$PBS-treated.

Peritonitis is considered an established model to study leukocyte chemotaxis and activation and expansion outside of the atherosclerotic lesion. We opted to use CCL5-dependent peritonitis, because CCL5 is one of the chemokines desensitized by GRK2, which at the same time is considered to be relevant in atherosclerosis. In vivo migration was measured after intraperitoneal injection of PBS (control) or CCL5 (RANTES; $200 \mathrm{ng} / \mathrm{mouse}$ ). CCL5 challenge led to increased peritoneal leukocyte presence compared with that in PBS treated animals for $G R K 2^{-/-}$chimeras $(P<0.05)$ but not for WT animals (Fig. $5 C$ ). In-depth analysis of monocyte migration into the peritoneal cavity of $\mathrm{GRK}^{-/}$chimeras showed a significant increased migration in CCL5challenged animals compared with that in PBS-treated animals (Fig. 5D).

\section{GRK2 Deficiency decreases Myeloid Hematopoiesis}

The observed effects of decreased GRK2 levels on the myeloid lineage and in particular on granulocytes and macrophages could reflect altered myelopoiesis. CFU assays with bone marrow cells from WT $(n=6)$ and $G R K 2^{-/-}$ mice $(n=5)$ showed that bone marrow from $G R K 2^{--}$mice contained fewer CFUs $(43.0 \pm 4.2$ vs. $57.2 \pm 2.8$ CFUs, $P=0.017$; Fig. $6 A$ ). This result was solely caused by a decreased number of G-CFUs in $G R K 2^{-/-}$chimeras $(21.4 \pm 1.0$ vs. $33.2 \pm 2.1$ G-CFUs, $P<0.001$; Fig. 6B); GM- and M-CFUs were unaffected. Thus, GRK2/bone marrow cells show an attenuated G-colony stimulating factor (CSF) response, explaining the decreased myeloid (monocytes/granulocytes) over lymphoid (B cells/T cells/NK cells) cell ratio in the circulation (Fig. 6C). 
A

D

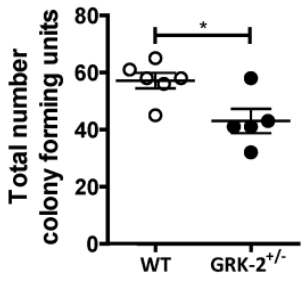

B

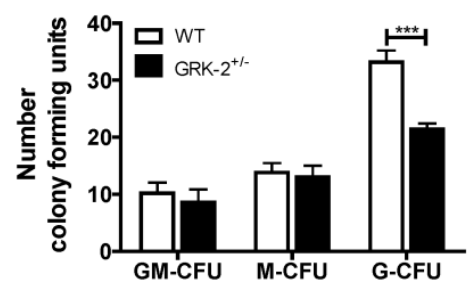

C

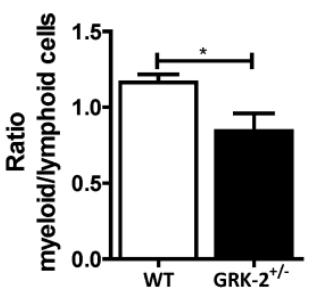

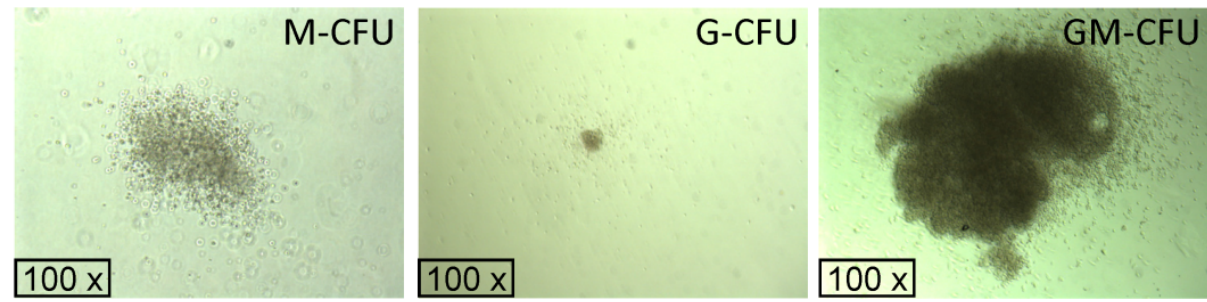

Figure 6 - Myeloid progenitor cells are decreased in $\mathrm{GRK}^{+/-}$bone marrow; which is associated with reduced blood monocyte and granulocyte numbers.

Colony forming unit (CFU) assays were performed on bone marrow from wild type and GRK2 ${ }^{+/-}$ donors. (A) The total number of colonies in WT control compared to heterozygous GRK2 chimeras. Based on morphological analysis Granulocyte/Macrophage-CFU (GM-CFU), Macrophage-CFU (M-CFU), and Granulocyte-CFU (G-CFU) were differentiated (C) Myeloid (granulocytes + monocytes) over lymphoid (B-cells + T-cells) ratio in the circulation of GRK2 ${ }^{+/-}$ deficient mice compared to controls. (D) Representative pictures showing Macrophage-CFU (MCFU), Granulocyte-CFU (G-CFU), and Granulocyte/Macrophage-CFU (GM-CFU) colonies (100x magnification). Wild type controls (white bars) and $\mathrm{GRK} 2^{+/-}$chimeras (black bars). ${ }^{*} p<0.05$; *** $p<0.001$

\section{Granulocyte and Macrophage GRK2 Deficiency does not explain Phenotype}

Because our results pointed to GRK2-deficient myeloid cells as the underlying cause for the phenotypic changes observed in the atherosclerotic lesion in $G R K 2^{-1}$ chimeras, we studied the effect of granulocyte-and macrophagespecific GRK2 deficiency in a LysM-Cre GRK2 $2^{\text {floxfflox }}$ bone marrow transplant model in $L D L r^{-1-}$ mice $(n=8 / 9)$. To our surprise, no difference was observed in total plaque area between LysM-Cre GRK2 $2^{\text {flox/flox }}$ and WT chimeras (Fig. 7A). In addition, necrotic core size was unchanged in these myeloid cell-specific GRK2deficient chimeras compared with that in controls (Fig. $7 B$ ), as were plaque collagen levels (Fig. 7C). Because the LysM-Cre GRK2 floxfllox model results in lowered macrophage GRK2 levels, we quantified intimal macrophage content in the aortic root lesions, revealing no differences between groups (Fig. 7D). 

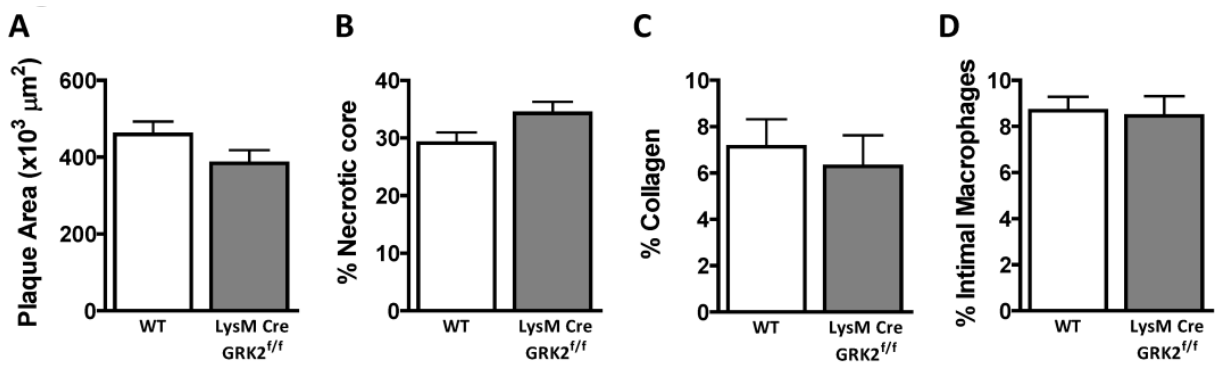

E
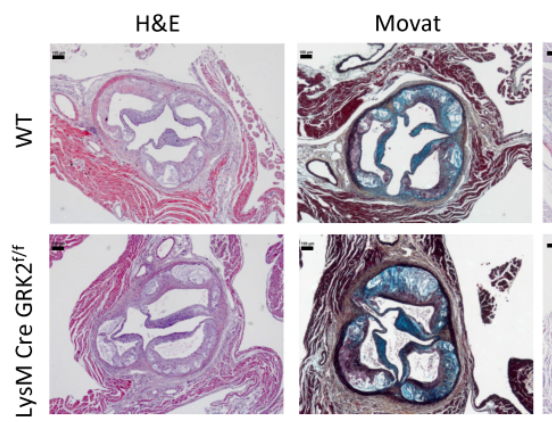

MAC-3

Figure 7 - Atherosclerotic plaque development and intimal macrophage accumulation are unaffected in LysM-Cre GRK2 ${ }^{\text {flox/flox }}$ chimeras.

(A) Plaque area and (B) necrotic core size were determined based on haematoxylin and eosin (H\&E) staining. (C) Collagen content was measured by analysis of Sirius Red staining under polarized light. (D) Macrophage content was measured in sections stained for the monocyte/macrophage marker MAC-3. (E) Representative pictures of H\&E, Movat's Pentachrome, and MAC-3 staining are shown for wild type and LysM Cre GRK2 ${ }^{\text {flox/flox }}$ chimeras. The top panels represent WT and the bottom panels LysM Cre GRK2 ${ }^{\text {flox/flox }}$ chimeras. Wild type controls (WT) are shown in white and LysM Cre GRK2 $2^{\text {flox/flox }}$ chimeras are shown in grey; scale bars are $100 \mu \mathrm{m}$.

In analogy with the $G R K 2^{-}$chimera study, flow cytometry of peripheral blood showed marked changes in granulocytes and Ly6C monocyte subsets in the granulocyte- and macrophage-specific GRK2-deficient chimeras (Table 1). These differences did not translate to plaque phenotypic effects. It appears that granulocyte- and macrophage-specific GRK2 deficiency does not affect atherogenesis, despite perturbed leukocyte patterns in circulation. The involvement of GRK2-deficient leukocytes, other than monocytes, cannot be excluded. However, total monocyte counts were not affected in LysM-Cre $G R K 2^{f l o x}$ fflox chimeras but were decreased in $G R K 2^{-\%}$ chimeras, indicating that monocytes, rather than granulocytes or macrophages, were probably the cell type responsible for the atheroprotective phenotype in hematopoietic GRK2 deficiency. 
Table 1 - Blood leukocyte composition is affected in macrophage/granulocyte specific GRK2 deficiency.

Leukocyte populations were determined in the blood from wild type (WT) and LysM-Cre GRK2 ${ }^{\text {flox/flox }}$ chimeras. Numbers shown are the relative percentages of living cells, except for CD4 and CD8 (\% of CD3 $\varepsilon^{+}$cells) and Ly6C high/low monocytes (\% of monocytes). ${ }^{*} p<0.05$ compared to WT.

\begin{tabular}{llc}
\hline & \multicolumn{1}{c}{ WT } & LysM-Cre GRK2f/f \\
\hline \% T-cells & $20.2 \pm 1.3$ & $18.6 \pm 0.9$ \\
\% CD4+ T-cells & $67.9 \pm 0.5$ & $69.4 \pm 0.6$ \\
\% CD8+ T-cells & $25.7 \pm 0.6$ & $24.7 \pm 0.2$ \\
\% B-cells & $22.1 \pm 2$ & $31 \pm 1.3^{*}$ \\
\% Granulocytes & $29.4 \pm 2$ & $24.5 \pm 0.8^{*}$ \\
\% Monocytes & $16.1 \pm 1.6$ & $15.8 \pm 0.5$ \\
\% Ly6C high monocytes & $56.5 \pm 1.3$ & $46.2 \pm 1.2^{*}$ \\
\hline \% Ly6C low monocytes & $33.8 \pm 1.9$ & $44.2 \pm 0.9^{*}$ \\
\hline
\end{tabular}

\section{Discussion}

A role of GRK2 in atherosclerosis has not yet been documented but could be inferred from its key regulatory role in CCL2 (monocyte chemotactic protein-1) and CCL5 (RANTES) function, which are instrumental in leukocyte chemotaxis and transmigration during atherogenesis ${ }^{20,31,38}$. Moreover, GRK2 levels were shown to be decreased in rheumatoid arthritis, an inflammatory disease sharing many features with atherosclerosis. Further, GRK2 expression was shown to be responsive to atherogenic cytokines such as IFN- $\Upsilon$ and IL- $6{ }^{\text {ref } 25,39}$. Our data identify a role for hematopoietic GRK2 in atherogenesis, excluding GRK2 deficiency in granulocytes and macrophages being responsible for the observed effects.

First, we mapped the effect of heterozygous hematopoietic GRK2 deficiency on atherosclerosis in $L D L^{-/}$mice. The donor mice were on a $\mathrm{C} 57 \mathrm{BL} / 6$ background but were $L D L r^{-/}$. LDLr expression in hematopoietic cells did not affect either repopulation of the hematopoietic lineage or atherosclerosis development and is widely accepted as the donor genotype in atherosclerosis research ${ }^{40}$. The $\mathrm{GRK}^{-/-}$genotype results in an approximately $50 \%$ reduction in GRK2 protein levels as shown before ${ }^{41}$. Homozygous GRK2-deficient mice could not be used in these experiments because GRK2 deficiency is embryonically lethal, and embryos do not survive beyond gestational day 15.5. Most likely the in utero mortality is caused by cardiac dysfunction ${ }^{33}$. Partial GRK2 deficiency resulted in reduced plaque burden and delayed plaque progression. In fact, $G R K 2^{-/-}$plaques did not progress beyond a fatty streak stage and contained more macrophages and intimal smooth muscle cells but 
showed profoundly diminished necrotic core formation. The latter pointed to a direct effect of GRK2 deficiency on macrophage phagocytosis or apoptosis. Indeed, GRK2 was previously shown to influence cytoskeleton function ${ }^{42,43}$, thereby potentially modifying macrophage phagocytic capacity. As shown previously, disrupted apoptotic cell clearance can accelerate atherosclerosis in mice ${ }^{44}$. However, analysis did not support the notion that the GRK2\%associated reduction in necrotic core size is due to increased phagocytosis, because apoptotic cell handling by macrophages was not affected.

Impaired necrotic core expansion is not caused by altered apoptosis of plaque macrophages. However, GRK2 ${ }^{-1-}$ macrophage necrosis in vitro was sharply attenuated after oxLDL exposure. The actual effect of macrophage death on atherosclerosis is still a matter of debate. Several studies have shown that in early and intermediate plaques, enhanced macrophage apoptosis is beneficial ${ }^{45,46}$, albeit others suggested otherwise ${ }^{47}$. In advanced atherosclerosis, the consensus is that macrophage death promotes necrotic core expansion and plaque destabilization ${ }^{48,49}$.

Enhanced plaque macrophage content and reduced lesion burden in $G R K 2^{-1-}$ chimeras could reflect changes in peripheral inflammation. GRK2 $2^{-1-}$ chimeras showed decreased monocyte counts. Total leukocyte numbers were increased, an effect that was mainly attributable to augmented granulocyte counts in circulation. Myelopoiesis, assessed by CFU assay, was attenuated in $G R K 2^{-/-}$bone marrow, and G-CFUs, in particular, were decreased. Although it remains to be established how GRK2 exactly interferes with G-CSF response at a molecular level, this process may be linked to granulocyte release or life span because granulocyte numbers also increased in the bone marrow. At a functional level, mobilization of macrophages to inflammatory sites seemed to be increased with partial GRK2 deficiency, which is compatible with the desensitizing effect of GRK2 on chemokine receptor activity ${ }^{50}$. Indeed, we could show that CCR5 chemokine receptor expression was increased on peripheral monocytes from $G R K 2^{-/}$chimeras. At a functional level, this increase was accompanied by increased monocyte migration in response to a CCL5 (RANTES) challenge, as shown in a model of chemokine-induced peritonitis. However, the roles of other cell types such as T lymphocytes, dendritic cells, and macrophages cannot be excluded, and they might contribute to the increased monocyte migration indirectly, because relevant, 
potentially GRK2-responsive chemokine receptors (CCR1, CCR2, and CCR7) are also expressed by lymphocyte subsets ${ }^{51,52}$.

The involvement of the chemokine receptor and ligand axis in regulating stromal release of $\mathrm{Ly} 6 \mathrm{C}^{\text {high }}$ monocytes from the bone marrow has already been shown for the CCL2/CCR2 axis ${ }^{53}$. However, the decreased Ly6C $\mathrm{C}^{\text {high }}$ monocyte levels in circulation and spleen are not likely to be due to GRK2dependent tuning of CCR2 egress because in that case increased monocyte levels would be expected. We did, however, observe that mobilization of monocytes is affected at some level because there are no differences observed in the bone marrow. On the other hand, mice deficient for CCL2 or CX3CR1 had decreased atherogenesis. Inhibition of the CCL5/CCR5 axis by treatment with a CCR5 antagonist (Met-CCL5) resulted in an even more dramatic reduction of atherogenesis ${ }^{54}$. A similar reduction in atherosclerosis development was observed in this study, indicating that GRK2-dependent processes other than receptor desensitization may be responsible for the phenotype observed.

Major effects of GRK2 deficiency on myeloid differentiation and mobilization have prompted us to address the contribution of granulocyte and macrophage GRK2 deficiency to the atheroprotection. The LysM-Cre $G R K 2^{f l o x / f l o x}$ transgenic mouse was previously shown to confer effective deletion efficiency in macrophages and granulocytes ${ }^{55,56}$. Contrary to our expectations, the LysM-Cre GRK2 $2^{\text {floxfflox }}$ bone marrow chimeras did not mirror the previously observed GRK2 $2^{-/-}$phenotype. In fact, LysM-Cre GRK2 $2^{\text {flox/flox }}$ chimeras completely lacked a plaque phenotype, disqualifying granulocyte and macrophage GRK2 deficiency as the major culprit, although the possibility that the phenotype of homozygous GRK2 deficiency differs from that of GRK2 ${ }^{-/}$cannot be excluded. On the other hand, in sepsis, it was recently shown that macrophage- and granulocyte-specific deficiency resulted in no clear phenotype attributable to these cells ${ }^{57}$. Flow cytometry of the LysM-Cre GRK2 $2^{\text {flox/flox }}$ chimeras and controls revealed a reduction in relative granulocyte abundance in blood of the $G R K 2^{f l o x}$ flox animals and in keeping the granulocyte- and macrophage-specific deletion, monocyte levels in circulation were not affected in the LysM-Cre GRK2 $2^{\text {floxflox }}$ model. It has previously been shown that the LysM expression in circulating monocytes is not sufficient to get the CRE levels necessary for effective deletion of the floxed gene, in this case GRK2 ${ }^{\text {ref }}{ }^{58}$. In contrast to this ineffective deletion in F4/80-negative immature macrophages or monocytes, the construct is highly efficient in the deletion of the floxed genes in F4/80- 
positive macrophages and in granulocytes ${ }^{59,60}$. Taken together, these data point to monocytes or potentially even T cells (albeit we did not observe overt effects of GRK2 deficiency on the number of circulating or plaque T cells) as responsible factors in the profound $G R K 2^{-/}$plaque phenotype.

Overall, our data indicate an equally complex and critical role of GRK2 in monocyte homeostasis and mobilization in the context of atherosclerosis. Partial hematopoietic GRK2 deficiency appeared to have a profound effect on atherogenesis. Whereas all data seem to pinpoint the myeloid lineage as the major effectors, reduced GRK2 levels in granulocytes and macrophages cannot be held directly accountable for this effect. Cumulatively, these data lead us to propose a major role for monocyte GRK2 in the observed phenotypic changes. It should be noted, however, that although we did not observe any major effects of $G R K 2^{--}$on other leukocyte subsets known to affect atherogenesis, such as $T$ lymphocytes and dendritic cells, the possibility that these subsets may nevertheless have modified the atherogenic response in an indirect manner cannot be excluded. Because even partial inhibition of GRK2 function already suffices to halt plaque progression at a fatty streak stage, our studies warrant further investigation into the perspective of GRK2 antagonists in atherosclerosis treatment and in determining the cell type responsible for effects on atherosclerosis development.

\section{Acknowledgements}

The authors thank Dr. M. Gijbels for her help in plaque morphology scoring. E.B. is the recipient of an Established Investigator Fellowship (grant 2003T201) of The Netherlands Heart Foundation. This work was supported in part by the U.S. National Institutes of Health (grants R01-NS003939 and NS074999 to A.K.). 


\section{Supplemental Figures}

A

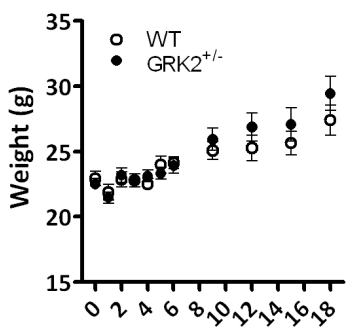

Time (weeks)
B

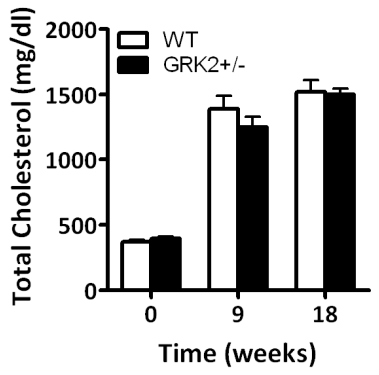

Supplemental figure 1 - Body weight development and cholesterol levels are unaffected in GRK2 $^{+/-}$chimeras.

(A) Body weight was monitored during the course of the experiment for WT (O) and GRK2 ${ }^{+-}(\bullet)$ chimeras. (B) Cholesterol levels were determined at baseline (week 0 ), week 9 , and prior to sacrifice (week 18), for WT (white bars) and GRK2 ${ }^{+/-}$(black bars) chimeras.

A

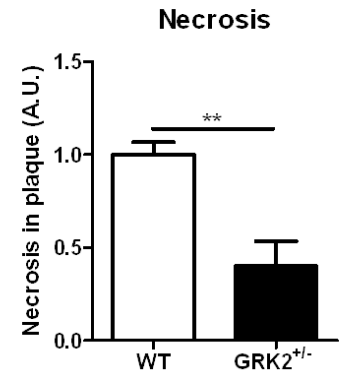

D

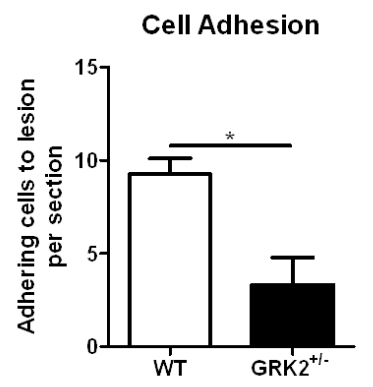

B

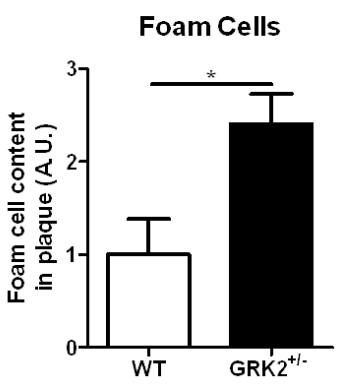

E

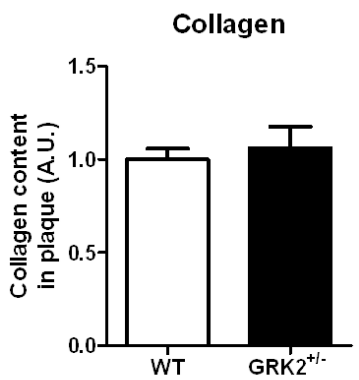

C

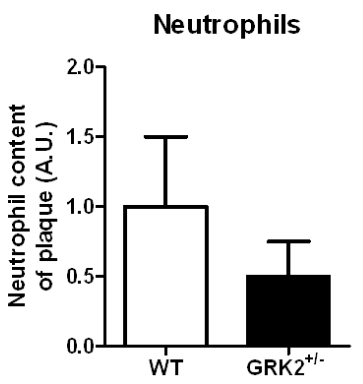

$\mathbf{F}$

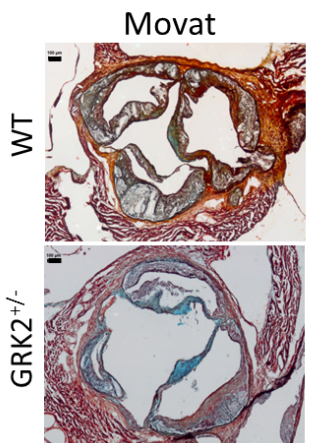

Supplemental figure 2 - Morphological scoring of atherosclerotic lesions.

Atherosclerotic lesions from WT and GRK2 ${ }^{+-}$chimeras were scored for (A) necrosis, (B) foam cell content, (C) neutrophil content, (D) cell adhesion, and (E) collagen by an independent operator based on Movat's Pentachrome staining. (F) Representative pictures for Movat's Pentachrome staining. WT controls (white bars) and GRK2 ${ }^{+/-}$chimeras (black bars); scale bars are $100 \mu \mathrm{m} .{ }^{*} p<$ $0.05, * * p<0.01$ 
A

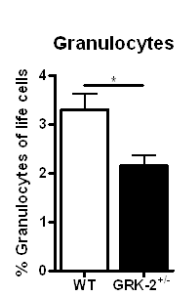

B

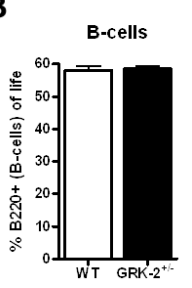

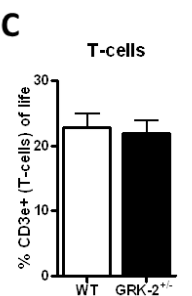
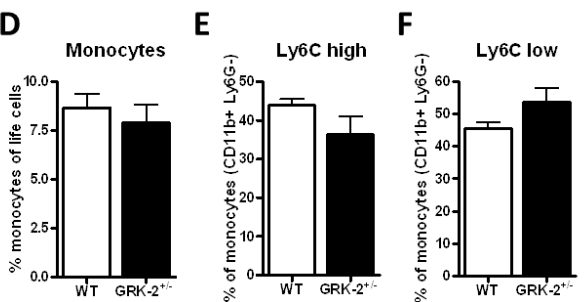

Supplemental figure 3 - Splenic leukocyte subsets.

Splenic (A) granulocytes, (B) B-cells, (C) T-cells, (D) monocytes, (E) Ly6C ${ }^{\text {high }}$ monocytes, and (F) Ly6C monocytes were determined in spleens from $\mathrm{GRK}^{+/-}$chimeras and WT controls. WT controls (white bars) and $\mathrm{GRK}^{+/-}$chimeras (black bars). ${ }^{*} p<0.05$ 

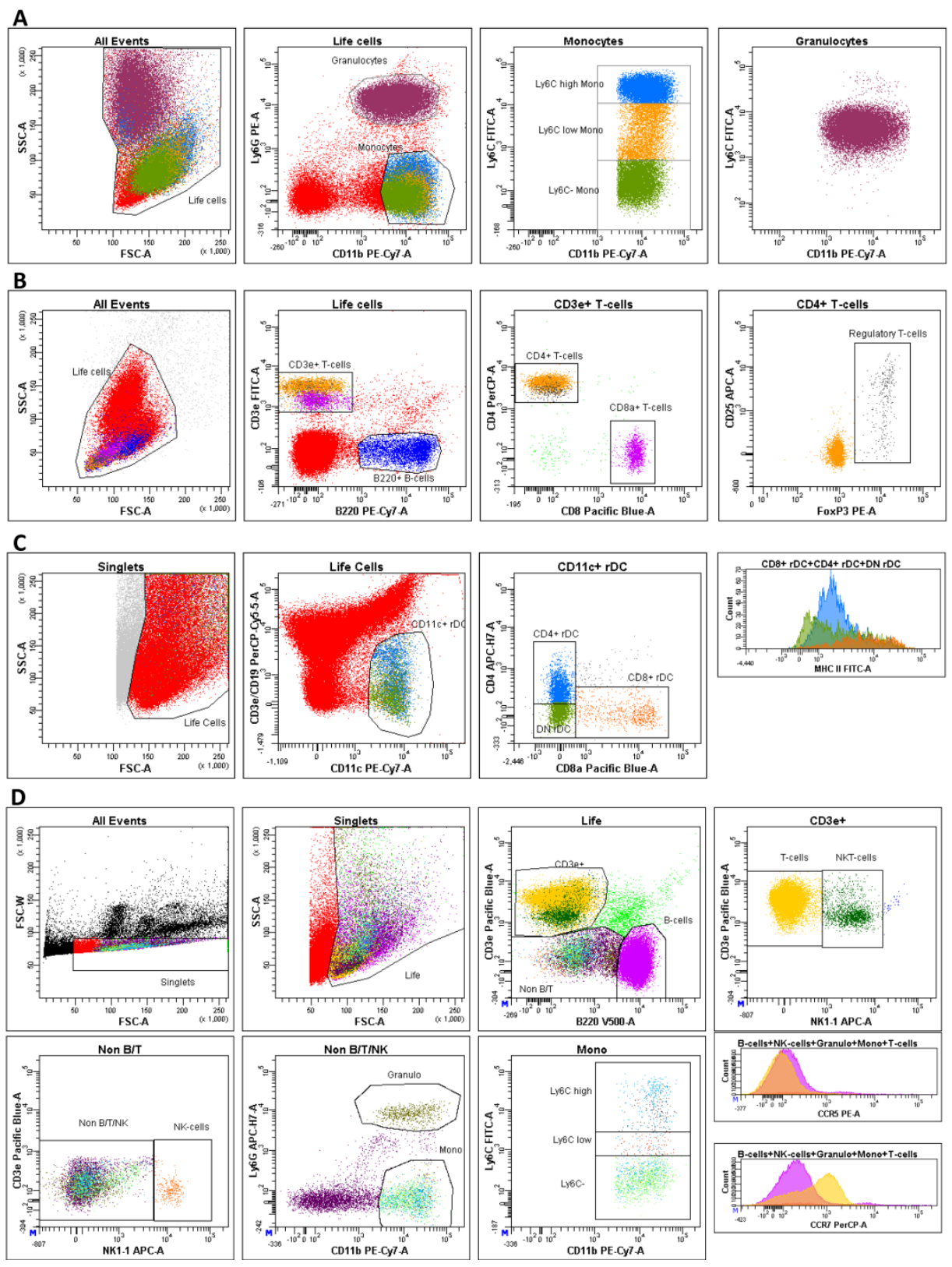

Supplemental figure 4 - Gating strategies for flow cytometry analyses.

(A) Monocytes/granulocyte gating in blood and spleen. (B) T-cell and B-cell gating in blood and spleen. (C) Resident dendritic cells (rDC) gating in spleen. (D) Chemokine receptor gating. 


\section{References}

1. Lusis AJ. Atherosclerosis. Nature. 2000;407(6801):233-241.

2. Braunersreuther V, Zernecke A, Arnaud C, et al. Ccr5 but not Ccr1 deficiency reduces development of diet-induced atherosclerosis in mice. Arterioscler Thromb Vasc Biol. 2007;27(2):373-379.

3. Rossi D, Zlotnik A. The biology of chemokines and their receptors. Annu Rev Immunol. 2000;18:217-242.

4. Bazan JF, Bacon KB, Hardiman G, et al. A new class of membrane-bound chemokine with a CX3C motif. Nature. 1997;385(6617):640-644.

5. Weber C, Schober A, Zernecke A. Chemokines: key regulators of mononuclear cell recruitment in atherosclerotic vascular disease. Arterioscler Thromb Vasc Biol. 2004;24(11):1997-2008.

6. Liehn EA, Zernecke A, Postea O, Weber C. Chemokines: inflammatory mediators of atherosclerosis. Arch Physiol Biochem. 2006;112(4-5):229-238.

7. Moser B, Willimann K. Chemokines: role in inflammation and immune surveillance. Ann Rheum Dis. 2004;63 Suppl 2:ii84-ii89.

8. Weber C. Platelets and chemokines in atherosclerosis: partners in crime. Circ Res. 2005;96(6):612-616.

9. Reape TJ, Groot PH. Chemokines and atherosclerosis. Atherosclerosis. 1999;147(2):213-225.

10. Lukacs NW, Strieter RM, Chensue SW, Kunkel SL. Activation and regulation of chemokines in allergic airway inflammation. J Leukoc Biol. 1996;59(1):13-17.

11. Burke-Gaffney A, Brooks AV, Bogle RG. Regulation of chemokine expression in atherosclerosis. Vascul Pharmacol. 2002;38(5):283-292.

12. Thelen M. Dancing to the tune of chemokines. Nat Immunol. 2001;2(2):129134.

13. Arai H, Charo IF. Differential regulation of G-protein-mediated signaling by chemokine receptors. J Biol Chem. 1996;271(36):21814-21819.

14. Muller S, Lohse MJ. The role of G-protein beta gamma subunits in signal transduction. Biochem Soc Trans. 1995;23(1):141-148.

15. Hausdorff WP, Caron MG, Lefkowitz RJ. Turning off the signal: desensitization of beta-adrenergic receptor function. FASEB J. 1990;4(11):2881-2889.

16. Attramadal $\mathrm{H}$, Arriza JL, Aoki $\mathrm{C}$, et al. Beta-arrestin2, a novel member of the arrestin/beta-arrestin gene family. J Biol Chem. 1992;267(25):17882-17890.

17. Arriza JL, Dawson TM, Simerly RB, et al. The G-protein-coupled receptor kinases beta ARK1 and beta ARK2 are widely distributed at synapses in rat brain. $J$ Neurosci. 1992;12(10):4045-4055.

18. Premont RT, Inglese J, Lefkowitz RJ. Protein kinases that phosphorylate activated G protein-coupled receptors. FASEB J. 1995;9(2):175-182.

19. Freedman NJ, Lefkowitz RJ. Desensitization of $\mathrm{G}$ protein-coupled receptors. Recent Prog Horm Res. 1996;51:319-351; discussion 352-313.

20. Vroon A, Heijnen CJ, Lombardi MS, et al. Reduced GRK2 level in T cells potentiates chemotaxis and signaling in response to CCL4. J Leukoc Biol. 2004;75(5):901-909. 
21. Penela P, Ribas C, Aymerich I, et al. G protein-coupled receptor kinase 2 positively regulates epithelial cell migration. EMBO J. 2008;27(8):1206-1218.

22. Weiss ER, Ducceschi MH, Horner TJ, Li A, Craft CM, Osawa S. Species-specific differences in expression of G-protein-coupled receptor kinase (GRK) 7 and GRK1 in mammalian cone photoreceptor cells: implications for cone cell phototransduction. $J$ Neurosci. 2001;21(23):9175-9184.

23. Chen X, Zhu H, Yuan M, Fu J, Zhou Y, Ma L. G-protein-coupled receptor kinase 5 phosphorylates p53 and inhibits DNA damage-induced apoptosis. J Biol Chem. 2010;285(17):12823-12830.

24. Vroon A, Kavelaars A, Limmroth V, et al. G protein-coupled receptor kinase 2 in multiple sclerosis and experimental autoimmune encephalomyelitis. J Immunol. 2005;174(7):4400-4406.

25. Lombardi MS, Kavelaars A, Schedlowski M, et al. Decreased expression and activity of G-protein-coupled receptor kinases in peripheral blood mononuclear cells of patients with rheumatoid arthritis. FASEB J. 1999;13(6):715-725.

26. Kavelaars A, Eijkelkamp N, Willemen HL, Wang H, Carbajal AG, Heijnen CJ. Microglial GRK2: a novel regulator of transition from acute to chronic pain. Brain Behav Immun. 2011;25(6):1055-1060.

27. Raake PW, Vinge LE, Gao E, et al. G protein-coupled receptor kinase 2 ablation in cardiac myocytes before or after myocardial infarction prevents heart failure. Circ Res. 2008;103(4):413-422.

28. Liu S, Premont RT, Kontos CD, Zhu S, Rockey DC. A crucial role for GRK2 in regulation of endothelial cell nitric oxide synthase function in portal hypertension. Nat Med. 2005;11(9):952-958.

29. Dinudom A, Fotia AB, Lefkowitz RJ, Young JA, Kumar S, Cook DI. The kinase Grk2 regulates Nedd4/Nedd4-2-dependent control of epithelial Na+ channels. Proc Natl Acad Sci U S A. 2004;101(32):11886-11890.

30. $\mathrm{Wu} \mathrm{JH}$, Zhang L, Fanaroff $A C$, et al. G protein-coupled receptor kinase-5 attenuates atherosclerosis by regulating receptor tyrosine kinases and 7transmembrane receptors. Arterioscler Thromb Vasc Biol. 2012;32(2):308-316.

31. Oppermann M, Mack M, Proudfoot AE, Olbrich H. Differential effects of CC chemokines on CC chemokine receptor 5 (CCR5) phosphorylation and identification of phosphorylation sites on the CCR5 carboxyl terminus. J Biol Chem. 1999;274(13):88758885.

32. Ishibashi S, Brown MS, Goldstein JL, Gerard RD, Hammer RE, Herz J. Hypercholesterolemia in low density lipoprotein receptor knockout mice and its reversal by adenovirus-mediated gene delivery. J Clin Invest. 1993;92(2):883-893.

33. Jaber $\mathrm{M}$, Koch WJ, Rockman $\mathrm{H}$, et al. Essential role of beta-adrenergic receptor kinase 1 in cardiac development and function. Proc Natl Acad Sci U S A. 1996;93(23):12974-12979.

34. Nijboer $\mathrm{CH}$, Heijnen CJ, Willemen HL, et al. Cell-specific roles of GRK2 in onset and severity of hypoxic-ischemic brain damage in neonatal mice. Brain Behav Immun. 2010;24(3):420-426.

35. Matkovich SJ, Diwan A, Klanke JL, et al. Cardiac-specific ablation of G-protein receptor kinase 2 redefines its roles in heart development and beta-adrenergic signaling. Circ Res. 2006;99(9):996-1003. 
36. Kanters E, Pasparakis M, Gijbels MJ, et al. Inhibition of NF-kappaB activation in macrophages increases atherosclerosis in LDL receptor-deficient mice. J Clin Invest. 2003;112(8):1176-1185.

37. De Rijke YB, Biessen EA, Vogelezang CJ, van Berkel TJ. Binding characteristics of scavenger receptors on liver endothelial and Kupffer cells for modified low-density lipoproteins. Biochem J. 1994;304 ( Pt 1):69-73.

38. Aragay AM, Mellado M, Frade JM, et al. Monocyte chemoattractant protein-1induced CCR2B receptor desensitization mediated by the $G$ protein-coupled receptor kinase 2. Proc Natl Acad Sci U S A. 1998;95(6):2985-2990.

39. Levine JD, Coderre TJ, Helms C, Basbaum Al. Beta 2-adrenergic mechanisms in experimental arthritis. Proc Natl Acad Sci U S A. 1988;85(12):4553-4556.

40. Herijgers N, Van Eck M, Groot PH, Hoogerbrugge PM, Van Berkel TJ. Effect of bone marrow transplantation on lipoprotein metabolism and atherosclerosis in LDL receptor-knockout mice. Arterioscler Thromb Vasc Biol. 1997;17(10):1995-2003.

41. Eijkelkamp N, Heijnen CJ, Willemen HL, et al. GRK2: a novel cell-specific regulator of severity and duration of inflammatory pain. J Neurosci. 2010;30(6):21382149.

42. Carman CV, Som T, Kim CM, Benovic JL. Binding and phosphorylation of tubulin by G protein-coupled receptor kinases. J Biol Chem. 1998;273(32):2030820316.

43. Cant SH, Pitcher JA. G protein-coupled receptor kinase 2-mediated phosphorylation of ezrin is required for $G$ protein-coupled receptor-dependent reorganization of the actin cytoskeleton. Mol Biol Cell. 2005;16(7):3088-3099.

44. Ait-Oufella $\mathrm{H}$, Kinugawa K, Zoll J, et al. Lactadherin deficiency leads to apoptotic cell accumulation and accelerated atherosclerosis in mice. Circulation. 2007;115(16):2168-2177.

45. Liu J, Thewke DP, Su YR, Linton MF, Fazio S, Sinensky MS. Reduced macrophage apoptosis is associated with accelerated atherosclerosis in low-density lipoprotein receptor-null mice. Arterioscler Thromb Vasc Biol. 2005;25(1):174-179.

46. Arai S, Shelton JM, Chen M, et al. A role for the apoptosis inhibitory factor AIM/Spalpha/Api6 in atherosclerosis development. Cell Metab. 2005;1(3):201-213.

47. Geng YJ, Libby P. Progression of atheroma: a struggle between death and procreation. Arterioscler Thromb Vasc Biol. 2002;22(9):1370-1380.

48. Han S, Liang CP, DeVries-Seimon $\mathrm{T}$, et al. Macrophage insulin receptor deficiency increases ER stress-induced apoptosis and necrotic core formation in advanced atherosclerotic lesions. Cell Metab. 2006;3(4):257-266.

49. Bot I, de Jager SC, Zernecke A, et al. Perivascular mast cells promote atherogenesis and induce plaque destabilization in apolipoprotein E-deficient mice. Circulation. 2007;115(19):2516-2525.

50. Jimenez-Sainz MC, Murga C, Kavelaars A, et al. G protein-coupled receptor kinase 2 negatively regulates chemokine signaling at a level downstream from $G$ protein subunits. Mol Biol Cell. 2006;17(1):25-31.

51. Loetscher P, Uguccioni M, Bordoli L, et al. CCR5 is characteristic of Th1 lymphocytes. Nature. 1998;391(6665):344-345. 
52. Hall SE, Mao A, Nicolaidou V, et al. Elucidation of binding sites of dual antagonists in the human chemokine receptors CCR2 and CCR5. Mol Pharmacol. 2009;75(6):1325-1336.

53. Serbina NV, Pamer EG. Monocyte emigration from bone marrow during bacterial infection requires signals mediated by chemokine receptor CCR2. Nat Immunol. 2006;7(3):311-317.

54. Combadiere $C$, Potteaux $S$, Rodero $M$, et al. Combined inhibition of CCL2, CX3CR1, and CCR5 abrogates Ly6C(hi) and Ly6C(lo) monocytosis and almost abolishes atherosclerosis in hypercholesterolemic mice. Circulation. 2008;117(13):1649-1657.

55. Willemen $\mathrm{HL}$, Eijkelkamp $\mathrm{N}$, Wang $\mathrm{H}$, et al. Microglial/macrophage GRK2 determines duration of peripheral IL-1beta-induced hyperalgesia: contribution of spinal cord CX3CR1, p38 and IL-1 signaling. Pain. 2010;150(3):550-560.

56. Patial S, Saini Y, Parvataneni S, et al. Myeloid-specific GPCR kinase-2 negatively regulates NF-kappaB1p105-ERK pathway and limits endotoxemic shock in mice. J Cell Physiol. 2011;226(3):627-637.

57. Parvataneni S, Gonipeta B, Packiriswamy N, Lee T, Durairaj H, Parameswaran N. Role of myeloid-specific G-protein coupled receptor kinase-2 in sepsis. Int J Clin Exp Med. 2011;4(4):320-330.

58. Goren I, Allmann N, Yogev N, et al. A transgenic mouse model of inducible macrophage depletion: effects of diphtheria toxin-driven lysozyme $\mathrm{M}$-specific cell lineage ablation on wound inflammatory, angiogenic, and contractive processes. Am J Pathol. 2009;175(1):132-147.

59. Cross M, Mangelsdorf I, Wedel A, Renkawitz R. Mouse lysozyme M gene: isolation, characterization, and expression studies. Proc Natl Acad Sci U S A. 1988;85(17):6232-6236.

60. Clausen BE, Burkhardt C, Reith W, Renkawitz R, Forster I. Conditional gene targeting in macrophages and granulocytes using LysMcre mice. Transgenic Res. 1999;8(4):265-277. 



\section{Abstract}

Increased cholesterol levels are a known risk factors for cardiovascular disease. Plaque macrophages accumulate oxidized cholesterol present in the atherosclerotic lesion. Despite the extensively described role of cholesterol in atherogenesis, little is known about the interaction between plasma cholesterol and circulating monocytes. Nowhere we analyzed not only the accumulation of lipoproteins and lipids in peripheral monocytes, but we also studied the effects of intracellular cholesterol accumulation on monocyte function to unravel processes in circulating monocytes contributing to atherogenesis. $L D L r^{--}$mice $(n=8)$ were fed a Western type diet (WTD) for up to three weeks to induce hypercholesterolemia and study the effects on early atherogenesis. After $0,0.5,1$, and 3 weeks of WTD diet animals were sacrificed and cholesterol accumulation was determined. Flow cytometry data showed increased granularity, which was caused by intracellular cholesterol/lipid accumulation as determined by electron microscopy and Oil-red-O staining. Plasma cytokine levels (IL-10, IL12p70, TNF $\alpha$, mKC, IL-6, and IL-1 $\beta$ ) were increased, with the most pronounced increase occurring in the first week of WTD. Increased granularity, and thus cholesterol accumulation, resulted in enhanced ROS production and increased monocyte rolling along and adherence to activated endothelium in mice on WTD. Taken together, we show that hypercholesterolemia induces lipid accumulation in circulating monocytes, thereby increasing both ROS production in these monocytes as well as stimulating monocyte-endothelium interactions. Overall, our data indicates that hypercholesterolemia in early atherosclerosis affects circulating monocytes and might contribute to unraveling novel pathways for intimal cholesterol accumulation through circulating monocytes. 


\section{Introduction}

Hypercholesterolemia is a well-known risk factor for the development of cardiovascular disease (CVD) and more in particular atherosclerosis, the main cause of CVD ${ }^{1,2}$. Indeed, increased levels of low density lipoprotein (LDL) cholesterol have been associated not only with increased CVD risk, but also plaque burden ${ }^{3}$. Classically, LDL is thought to permeate into the atherosclerotic plaque and after modification into oxidized LDL (oxLDL). Cells from the myeloid lineage, in particular monocyte-derived macrophages, will scavenge the oxLDL present in the lesion, identifying infiltrating monocytes and plaque macrophages as a major player in atherosclerosis.

Monocytes can be divided into two distinct subsets based on the expression of specific surface markers. In humans inflammatory CD $14^{\text {high }} / C D 16$ and patrolling $\mathrm{CD} 14^{\mathrm{low}} / \mathrm{CD} 16^{\text {t+ }}$ monocytes have been described ${ }^{4}$. In mice monocytes are separated based on Ly6C expression into inflammatory Ly6 $\mathrm{C}^{\text {high }}$ and patrolling Ly6 $6 \mathrm{C}^{-}$monocytes ${ }^{5,6}$. CC-motif chemokine receptor 2 (CCR2) is expressed at high levels by inflammatory monocytes ${ }^{7}$, whereas $\mathrm{CX}_{3} \mathrm{C}$-motif chemokine receptor $1\left(\mathrm{CX}_{3} \mathrm{CR} 1\right)$, or fractalkine receptor, is more abundant on patrolling monocytes ${ }^{8,9}$. Inflammatory monocytes are generally thought to be the most important subtype in early atherogenesis, being efficiently recruited to the lesion in a CCR2 dependent manner ${ }^{10}$. However experimental loss-offunction studies as well as expression studies were supportive of a role for patrolling monocytes as well, at later stages of disease progression ${ }^{11}$. Nevertheless, the exact role of both subsets in plaque macrophage accumulation and plaque progression has not yet been elucidated completely.

Accumulation of cholesterol in macrophages is mediated by scavenger receptors such as SR-A and CD36 ${ }^{12,13}$. In atherosclerosis, the efflux of oxLDL from macrophages via ATP binding cassette $(A B C)$ transporters, $A B C A 1$ and $A B C G 1$, is hampered causing progressive intracellular buildup of free cholesterol and eventually cholesterol crystals ${ }^{14,15}$. Both intracellular cholesterol, as well as free cholesterol in the lesion, can trigger several proinflammatory pathways, contributing to the progression of early atherosclerotic lesions. Main downstream signaling pathways triggered upon (ox)LDL dependent macrophage activation are NF- $\beta \beta$ signaling via toll-like receptor (TLR) 4 and TLR $6{ }^{16}$, ERK signaling via TLR2/TLR4, and ER stress ${ }^{17-19}$. Besides the effects described for oxLDL, also cholesterol crystals can trigger inflammatory signaling via the inflammasome through lysosomal damage ${ }^{20,21}$. 
LDL cholesterol can also affect arterial endothelial cell function, upregulating integrins like intercellular adhesion molecules (ICAMs) ${ }^{22}$ and vascular cell adhesion molecule (VCAM) $1^{23}$ as well as inducing monocyte chemotactic molecule secretion (e.g. CXCL1 and CCL2) ${ }^{19,24,25}$. Although ample evidence points towards increased monocyte adhesion and transmigration under hypercholesterolemic conditions ${ }^{26,27}$, this effect was generally attributed to endothelial activation, not to direct impact of hyperlipidemia on monocytes.

Nevertheless, hypercholesterolemia, in combination with reduced cholesterol efflux, is known to induce monocytosis in animal models such as $L D L$ receptor deficient $\left(L D L r^{--}\right)$and apolipoprotein $E$ deficient $\left(A p o E^{--}\right)$mice, but also in patients with familial hypercholesterolemia $(\mathrm{FH})^{28}$, or non-genetic hypercholesterolemia ${ }^{29}$. This phenomenon could be attributable to intracellular cholesterol accumulation induced lipid raft formation, stimulating proliferation of myeloid progenitors via IL-3 and granulocyte/macrophagecolony stimulating factor (GM-CSF) signaling ${ }^{30,31}$. In the current study we investigated implications of acute hypercholesterolemia on differentiation, function, and dynamics of circulating monocytic cells. Pro-atherogenic $\left(L D L r^{-/}\right)$ mice were monitored after onset of Western type diet (WTD) feeding and monocyte subsets were analyzed.Our study shows that circulating monocytes rapidly accumulate cholesterol in situ, resulting in diet-induced functional aberrations.

\section{Materials \& Methods}

\section{Animals}

Male low density $L D L r^{-1}$ mice were obtained from the animal breeding facility at Maastricht University (Maastricht, The Netherlands). Mice were fed a regular chow diet (RM3; Special Diet Services [SDS], Essex, UK) or a Westerntype diet (WTD; $0.25 \%$ cholesterol, $15 \%$ cocoa butter, $1 \%$ corn oil, $40.5 \%$ sucrose, $10 \%$ corn starch, and 5,95\% cellulose; SDS). Drinking water and food were provided ad libitum. Experiments were performed at the Maastricht University animal facility. All experimental protocols were approved by the Maastricht University ethics committee for animal experiments. 


\section{In vivo monocyte-endothelial cell interaction measurement}

Monocyte-endothelial cell interactions were measured in male $L D L r^{-1}$ mice either on normal chow $(n=3)$ or WTD $(n=3)$. Alexa568-coupled anti-CD115 antibody ( $5 \mu \mathrm{g} /$ mouse, eBioscience) and FITC-conjugated anti-CD31 antibody ( $35 \mu \mathrm{g} /$ mouse, BD Bioscience) were administered intravenously. Immediately hereafter, mice were anesthetized by a dorsal subcutaneous injection with ketamine $(100 \mathrm{mg} / \mathrm{kg}$ body weight, Nematek) and xylazine $(10 \mathrm{mg} / \mathrm{kg}$ body weight, Sedamun). Subsequently, mice were prepared for multiphoton laser scanning microscopy (MPLSM) by exposing the sternohyoid muscle by dissection. MPLSM imaging was performed on a Leica SP5 imaging platform (Leica Microsystems, Germany) that integrates multiphoton microscopy with fast resonant scanning, and uses a Compact Ultrafast Ti:Sapphire Laser (Chameleon, Coherent, USA). An excitation wavelength of $820 \mathrm{~nm}$ was used in all experiments. Tissue was observed using a 20x NA 1.0 water immersion objective (HCX PL APO L, Leica Microsystems) with a numerical aperture of 1.00 and an integrated optical zoom mechanism allowing magnification up to 60x. Photo-multiplier tubes (PMT) were used to detect three spectral regions: PMT1: 500 - 550; PMT2: 565 - 605. In vivo MPLSM imaging was performed at a frame size of $400 \times 400$ pixels (pixel size: $1.0882 \times 1.0882 \mu \mathrm{m}$ ), scanning at $8000 \mathrm{~Hz}$ for fast recording ( 20 frames/sec) and using a line average of 2 (10 frames $/ \mathrm{sec}$ ) for more detailed scans. Per time series a total of 500 frames was recorded. After in vivo imaging, the animal was sacrificed and additional in situ imaging was performed to examine the vasculature at higher resolution and an increased signal-to-noise ratio without motion artifacts. In situ imaging was performed at a frame size of $512 \times 512$ pixels (pixel size: $0.847 \times 0.847 \mu \mathrm{m}$ ), scanning at $200 \mathrm{~Hz}$, and an interplanar distance of $1.00 \mu \mathrm{m}$.

\section{Flow cytometry}

Blood, spleen, and bone marrow were collected upon sacrifice. Absolute leukocyte counts in blood were obtained using BD Trucount tubes, according to the manufacturers protocol (BD Biosciences). In short, Fc-receptor blocking antibody was added to the Trucount tubes. Subsequently, $50 \mu \mathrm{L}$ anticoagulated whole blood was added and the tube was gently vortexed. After incubation for ten minutes, the antibody cocktail (supplemental table 1) was added and the suspension was incubated at room temperature in the dark for 
an additional 20 minutes. Finally, hypotonic lysis buffer was added and after 15 minutes samples were analyzed.

Myeloid progenitor densities were measured in spleen and bone marrow. Single cell suspensions were made from spleen by crushing the tissue over a $70 \mu \mathrm{m}$ cell strainer (BD Biosciences, NJ, USA). One femur and one tibia per mouse were flushed with ice-cold PBS and single cell suspensions were prepared using a $70 \mu \mathrm{m}$ cell strainer (BD). subsequently, spleen and bone marrow cells were stained with biotin-conjugated antibodies against lineage (Lin) markers (CD5, CD45RA [B220], CD11b, Gr-1 [Ly6C/G], 7-4, and Ter-119; Miltenyi Biotec) and $\operatorname{Lin}^{+}$positive cells were depleted using streptavidin conjugated magnetic beads (Miltenyi Biotech) and LS columns (Miltenyi Biotech). Lin cell pools were stained with antibodies for Sca-1, c-Kit (CD117), CD34, CD16/32, CD115, and lineage markers (CD5, CD45RA [B220], CD11b, Ly6C, Ly6G, Ter-119).

Mature leukocytes in spleen and bone marrow were analyzed after initial removal of erythrocytes by incubation with hypotonic lysis buffer (8.4 g $\mathrm{NH}_{4} \mathrm{Cl}$ and $0.84 \mathrm{~g} \mathrm{NaHCO}_{3}$ per liter distilled water). Non-specific Fc-receptor binding was blocked by the addition of anti-CD16/CD32 antibody (eBioscience, San Diego, USA). Subsequently, single cell pools were stained with antibody mix (supplemental table 1).

Intracellular interleukin-1 production was measured in blood leukocyte subsets. After lysis of erythrocytes, leukocyte pools were incubated for 4 hours in RPMI-1640 medium (supplemented with $10 \%$ fetal calf serum and $0.1 \%$ Golgiplug $[B D])$ to induce intracellular cytokine accumulation. Cells were resuspended in FACS buffer and after FC-receptor block, surface markers were labeled with antibodies against CD3ع, CD45RA (B220), NK1.1, CD11b, Ly6G, and Ly6C. Subsequently, cells were fixed using Fix/Perm solution (eBioscience). Next, cells were permeabelized and stained with interleukin (IL) $1 \alpha, \mathrm{IL}-1 \beta$, and pro-IL-1 antibodies.

Intracellular reactive oxygen species accumulation in blood and splenic leukocyte subsets were determined by staining of cells with $100 \mu \mathrm{L} 20 \mu \mathrm{M}$ DCFDA in PBS and incubated for 20 minutes at $37^{\circ} \mathrm{C}$. Cells were washed once in PBS and resuspended in FACS buffer. After Fc-receptor block, surface markers were labeled with antibodies against CD3e, CD45RA (B220), NK1.1, CD11b, Ly6G, and Ly6C and analyzed. 
All samples and buffers were kept on ice throughout the experiment unless indicated otherwise. All measurements were performed on a FACS Canto II (BD Biosciences) and analysis of acquired data was performed using FACS Diva software (BD Biosciences).

\section{FACS Sorting}

Upon sacrifice blood and spleen were collected and single cell suspensions were prepared. Fc-receptor blocking antibody was added to the cell suspension. Subsequently, antibodies against CD3ع, CD45RA (B220), NK1.1, $\mathrm{CD} 11 \mathrm{~b}$, Ly6G, and Ly6C were added and incubated at $4^{\circ} \mathrm{C}$ in the dark for 30 minutes. Finally, monocyte Ly6C subsets were sorted in RPMI-1640 medium supplemented with $10 \%$ FCS on a FACS ARIA flow cytometer (BD Biosciences).

\section{Cytokine multiplex}

Plasma samples were frozen and stored at $-80^{\circ} \mathrm{C}$ until further use. Samples were thawed and selected cytokines mKC (CXCL1), tumor necrosis factor (TNF) $\alpha, I L-1 \beta, I L-6, I L-10, I L-12 p 70$, and interferon (IFN) $\gamma$ were measured using Multi-array electrochemiluminescence platform (K15012A-5, detection range $2.4 \mathrm{pg} / \mathrm{ml}$ to $10,000 \mathrm{pg} / \mathrm{ml}$; MesoScaleDiscovery, Gaithersburg, MD, USA). Analysis was performed as described by Van Bussel et $a l^{32}$. Each sample was analyzed in duplicate on the same array plate.

\section{Electron microscopy}

Monocytes were FACS sorted and fixed in 3\% glutaraldehyde (GTA; Ted Pella, Redding, $\mathrm{CA}, \mathrm{USA})$ in $0.1 \mathrm{M}$ phosphate buffer $(\mathrm{pH}=7.6)$ for 15 minutes. After several washes, cells were post-fixed in $1 \%$ osmium tetroxide solution and routinely dehydrated through $100 \%$ ethanol, cleared with propylene oxide, and embedded in epoxy resin. Ultra-thin sections ( $70-90 \mathrm{~nm}$ ) were cut on an ultramicrotome, mounted on Formvar (1595 E, Merck) coated 75 mesh copper grids, and counterstained with uranyl acetate and lead citrate before analysis on a Philips CM100 transmission electron microscope.

\section{Oil-red-O staining}

FACS sorted monocytes were spotted on glass slides using Cyto-Tek centrifuge (Sakura Finetek, Torrance, CA, USA). Cells were fixed in $4 \%$ formalin for 10 minutes and subsequently washed twice in PBS. Next, slides were incubated for 15 minutes in $60 \%$ isopropanol, followed by $60 \%$ Oil-red-O solution for 20 
minutes. Cells were washed gently with $60 \%$ isopropanol and once with water. Finally, the cells were counterstained with haematoxylin and slides were mounted with aquamount. Pictures were made at 40x magnification (Leica).

\section{Statistical Analysis}

Student's $t$ test was used; nonparametric data were analyzed using a MannWhitney $U$ test. All analyses were performed using GraphPad Prism 5 software (GraphPad Software Inc., LA Jolla, CA, USA), and values of $P<0.05$ were considered statistically significant.

\section{Results}

\section{Monocyte intra-cellular complexity is increased upon WTD}

Flow cytometry analysis of whole blood from mice on WTD showed that the monocyte fraction displayed markedly increased side scatter (SSC) compared to chow fed control animals as early as half a week of WTD feeding (figure 1A). As expected, plasma cholesterol levels progressively increased upon onset of WTD feeding to plateau at 3 weeks (figure 1B). Of note, the average monocyte SSC correlated to the plasma cholesterol levels $\left(R^{2}=0.726 ; p<0.0001\right.$; data not shown).

A

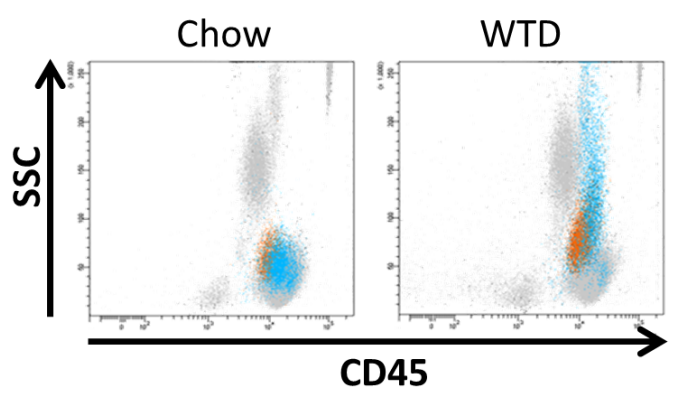

B

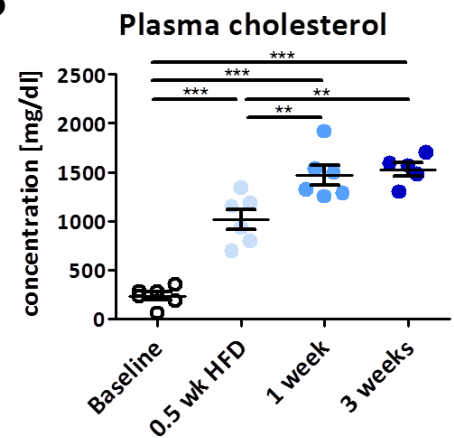

Figure 1 - Monocyte granularity correlates to plasma cholesterol levels

(A) Representative dot plots indicating increased side scatter profile of monocytes (orange and blue) from $L D L r^{-/}$mice on normal chow or 3 weeks Western type diet (WTD). (B) Plasma cholesterol levels measured at baseline (normal chow), and after $0.5,1$, and 3 weeks of WTD feeding. ${ }^{* *} p<0.01$ and ${ }^{* * *} p<0.001$ 
Next, we investigated whether WTD induced SSC changes manifested in both inflammatory Ly $6 \mathrm{C}^{\text {high }}$ and patrolling Ly $6 \mathrm{C}^{-}$monocytes. Interestingly, the enhanced scattering was predominantly observed in Ly6C monocytes (figure $2 A+B)$. Inflammatory Ly6 $C^{\text {high }}$ monocytes displayed significantly increased side scattering (granularity), albeit much less pronounced, than that of $\mathrm{Ly}^{-} \mathrm{C}^{-}$ monocytes (figure $2 \mathrm{~A}+\mathrm{B}$ ).

To pinpoint the cause of this increased monocyte granularity, Ly6C and Ly6 $\mathrm{C}^{\text {high }}$ monocytes were sorted and electron microscopic (EM) images were made. EM analysis indicated the overt presence of lipid droplets and even cholesterol crystals in circulating monocytes from WTD fed animals, but not chow fed animals (figure $2 \mathrm{C}$ ). Additional, Oil-red-O staining confirmed that monocytes, both Ly6C and Ly6 $\mathrm{C}^{\text {high }}$, contained lipid droplets (figure $2 \mathrm{C}$ ).

\section{Hypercholesterolemia induces leukocytosis}

Total leukocyte levels were up by almost $45 \%$ in WTD compared to chow fed mice (figure $3 \mathrm{~A}$ ). This increment could be ascribed to elevated granulocyte (figure $3 \mathrm{~B}$ ) and monocyte levels (figure $3 \mathrm{C}$ ), which in fact more than doubled in WTD fed animals, confirmative of previous data on hypercholesterolemia induced monocytosis ${ }^{30,31}$. Furthermore, the Ly $6 \mathrm{C}^{\text {high }}$ to $\mathrm{Ly} 6 \mathrm{C}^{-}$ratio was doubled after 3 weeks of WTD (figure $3 D+E$ ). Marked leukocytosis and increased monocyte granularity were also observed in spleen (supplemental figure $1 \mathrm{~A}-\mathrm{E}$ ) and bone marrow (supplemental figure 2A-D) of $L D L r^{-/}$animals after 3 weeks of WTD. Despite a small reduction in LK-cells, no significant effects could be observed on erythrocyte/megakaryocyte progenitors (EMP), common myeloid progenitors (CMP), or granulocyte/macrophage progenitors (GMP) after 3 weeks WTD feeding compared to normal chow (supplemental figure $2 \mathrm{E}+\mathrm{F}$ ). 
A

Granularity Circulating Monocytes

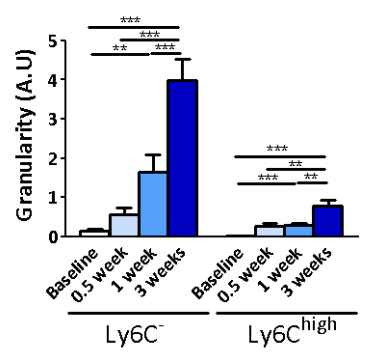

B
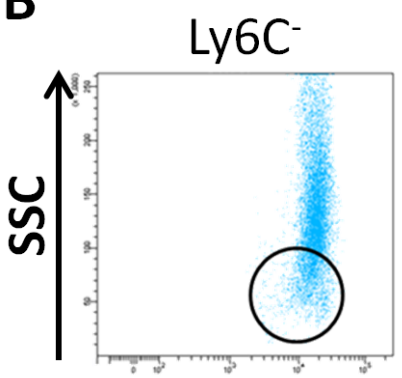

Ly6Chigh

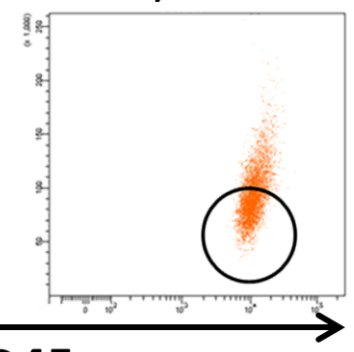

CD45

C

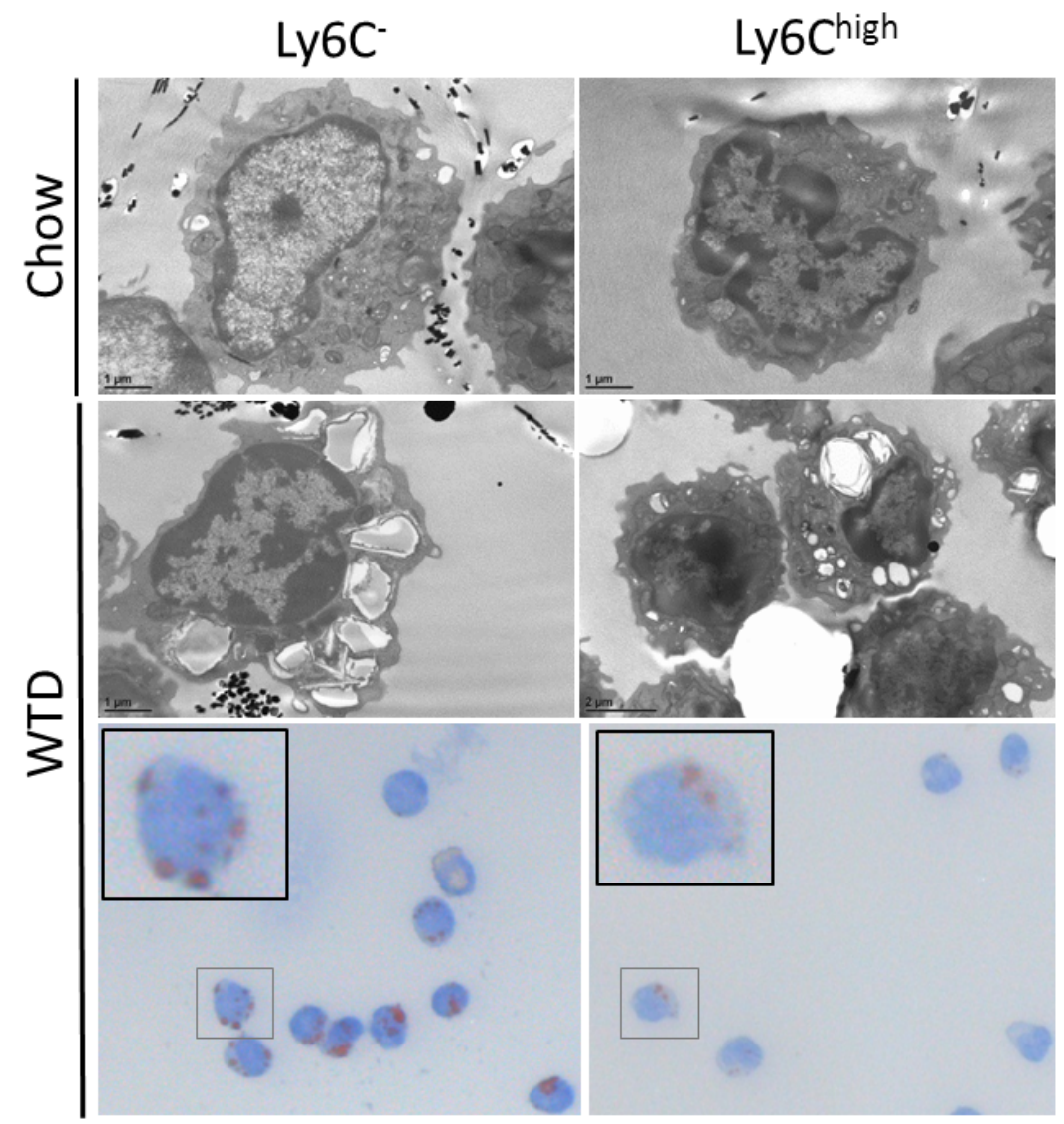

Figure 2 - Lipid accumulation is responsible for increased monocyte granularity

(A) Monocyte granularity was determined for Ly6C and Ly6C ${ }^{\text {high }}$ monocytes at baseline (normal chow, and after 0.5, 1, and 3 weeks WTD feeding. (B) Representative dot plots indicative of Ly6C and Ly $6 C^{\text {high }}$ monocyte granularity. Circles depict outline of populations under chow conditions. (C) Representative electron microscopy (EM) images for Ly6C (left column) and Ly6C ${ }^{\text {high }}$ (right column) monocytes on normal chow (top row) or WTD (middle row). Bottom panels show representative images for Oil-Red-O staining. 
A

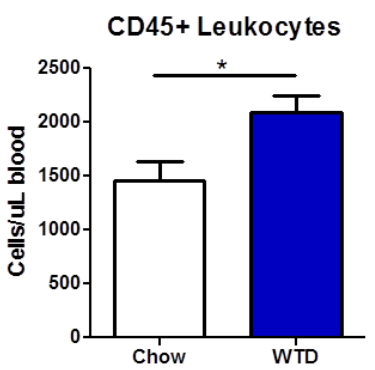

D

Monocyte subsets

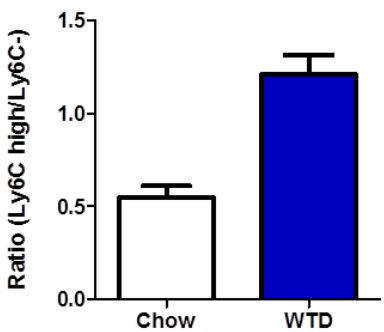

B

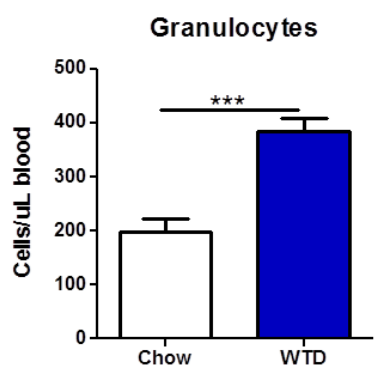

$\mathbf{E}$

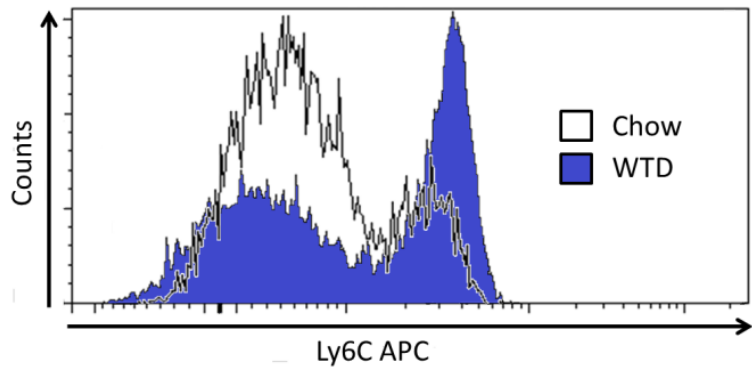

Figure 3 - Circulating leukocyte levels are increased upon cholesterolemia

Absolute leukocyte levels were measured in blood upon sacrifice using BD TruCount tubes. (A) Total $\mathrm{CD}_{4} 5^{+}$leukocytes, (B) $\mathrm{CD} 11 \mathrm{~b}^{+} / \mathrm{Ly}_{6 \mathrm{G}^{+}}$granulocytes, (C) $\mathrm{CD} 11 \mathrm{~b}^{+} / \mathrm{Ly}_{6 \mathrm{G}}$ monocytes, and (D) Ly6C monocyte subsets were measured. (E) Representative histograms indicating Ly6C and Ly $6 C^{\text {high }}$ monocytes in chow fed and WTD fed animals. ${ }^{*} p<0.05$ and ${ }^{* * *} p<0.001$

\section{Cytokine profile indicates systemic diet-induced inflammatory profile}

Plasma cytokine levels increased rapidly after the onset of WTD. After three to seven days, cytokine levels of IL-10, IL-12p70, IFN- $\Upsilon$, IL-1 $1 \beta, \mathrm{mKC}$, and IL-6 on average doubled compared to baseline values (normal chow) (figure 4A-F). TNF plasma levels were either not detectable or just above the detection limit. While elevated IL-10, IL12p70, and IFN- $\gamma$ levels, and to a lesser extend IL-6, persisted for 3 weeks (figure 4A-D). Plasma levels of the pro-inflammatory cytokines IL-1 $\beta$ and $\mathrm{mKC}$ almost reverted to baseline after 3 weeks of WTD feeding (figure $4 \mathrm{E}+\mathrm{F}$ ). 


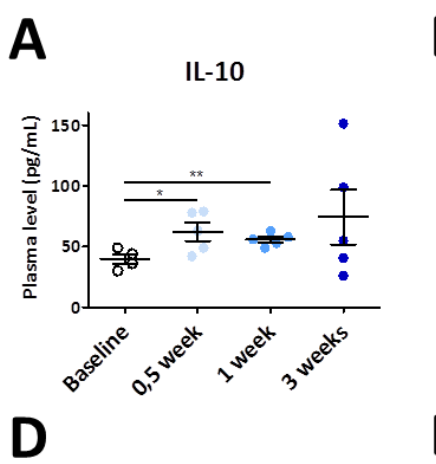

IL-6

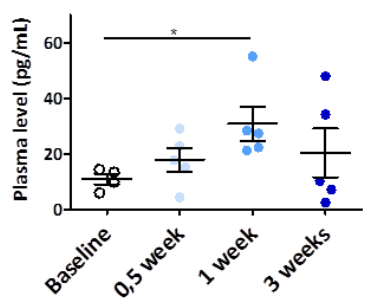

IL-12p70

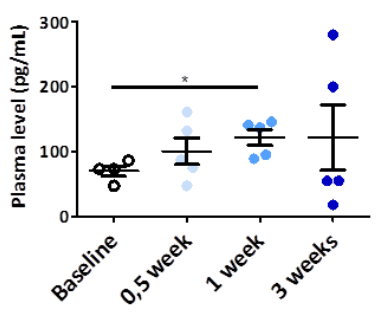

IL-1 $\beta$

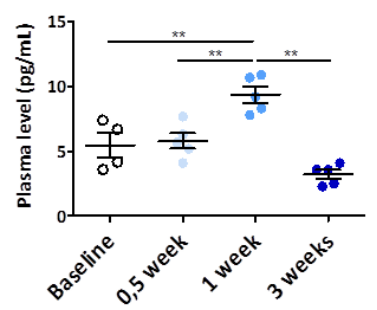

IFN-y

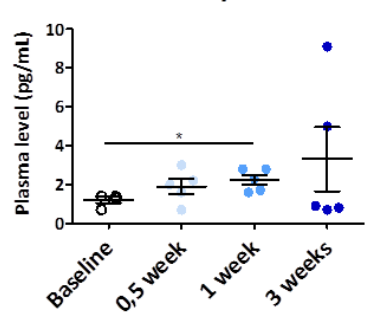

$\mathbf{F}$

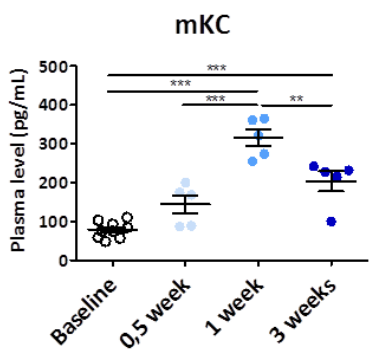

Figure 4 - Cytokine levels increase in response to hypercholesterolemia

Plasma cytokine levels were measured for (A) IL-10, (B) IL-12p70, (C) IFN- $\gamma$, (D) IL-6 (E) IL-13, and (F) $\mathrm{mKC}$ at baseline (normal chow; open circles) and after 0.5 week (pale blue), 1 week (light blue), and 3 weeks (dark blue) of WTD. ${ }^{*} p<0.05 * * p<0.01$ and ${ }^{* * *} p<0.001$

\section{Intra-cellular lipoproteins trigger ROS production}

In macrophages intra-cellular lipid accumulation was previously reported to trigger the production of reactive oxygen species in an ER stress and ERKsignaling dependent manner ${ }^{19}$. Since, we observed similar lipid accumulation in circulating monocytes from WTD fed $L D L r^{-1}$ mice, we analyzed ROS production in these monocytes. Indeed, both high granular Ly $6 \mathrm{C}^{-}$and Ly $6 \mathrm{C}^{\text {high }}$ monocytes had significantly increased levels of ROS compared to their low granular counterparts (figure $5 \mathrm{~A}+\mathrm{B}$ ). The amount of intra-cellular lipids did not appear to affect the ROS production as the levels in Ly6C and Ly6 $\mathrm{C}^{\text {high }}$ high granular monocytes was comparable. As Ly6 $\mathrm{C}^{\text {high }}$ monocytes showed less accumulation of intra-cellular lipids this subset conceivably is more susceptible to lipid associated ROS formation. 
A

ROS circulating Ly6C' mono
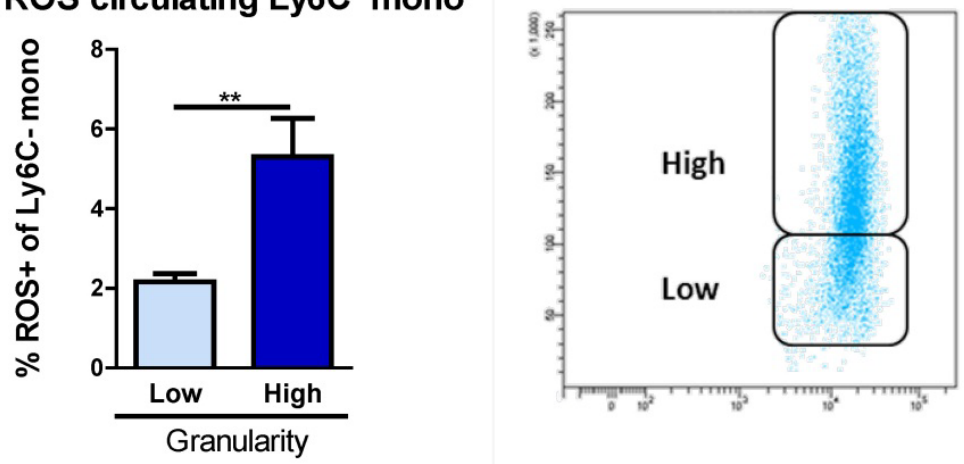

B

ROS circulating Ly6C ${ }^{\text {high }}$ mono
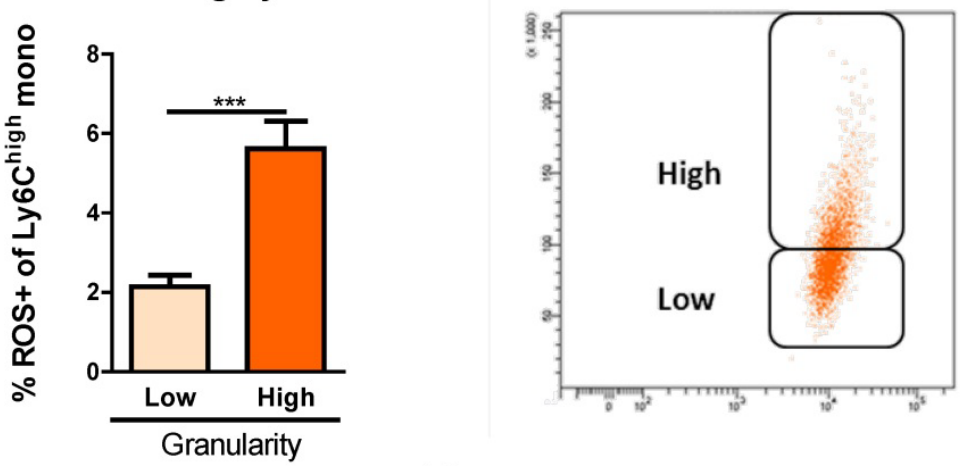

C

IL-1 $\beta$

D Ly6C' monocytes

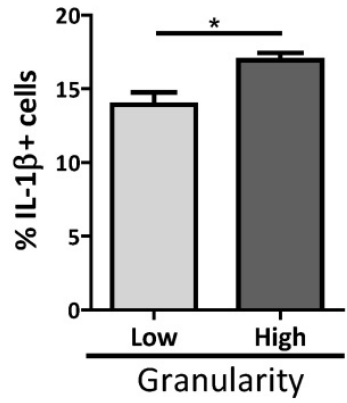

\section{IL-1 $\beta$ Ly6C $^{\text {high }}$ monocytes}

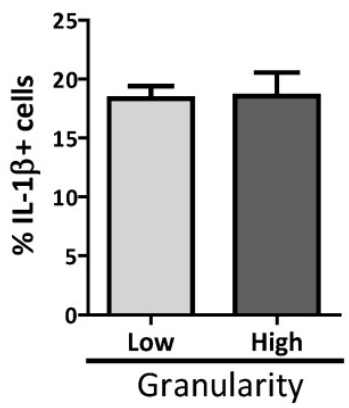

Figure 5 - Reactive oxygen species are augmented in all high granular monocytes, while IL-1 $\beta$ levels are only affected in high granular Ly6C monocytes

Reactive oxygen species (ROS) were measured in (A) Ly6C and (B) Ly6 $\mathrm{C}^{\text {high }}$ monocytes for both the low granular and high granular fractions, based on normal chow gating control. In the dot plots the gating for the low and high granular monocyte subsets are indicated. IL- $1 \beta$ levels were measured in (C) Ly6C and (D) Ly6C ${ }^{\text {high }}$ monocytes for both the low granular and high granular fractions. ${ }^{*} p<0.05^{* *} p<0.01$ and ${ }^{* * *} p<0.001$ 
As shown by Latz et al, macrophage cholesterol accumulation induces inflammasome activation. We therefore assessed intracellular production of the inflammasome cytokine IL-1 $\beta$ in monocytes from mice on WTD for 3 weeks. High granular Ly6C monocytes had significantly increased IL-1 $\beta$ levels compared to low their granular counterparts (figure $5 \mathrm{C}$ ). In contrast, Ly6C ${ }^{\text {high }}$ monocytes did not show any difference in intra-cellular IL-1 $\beta$ levels between low and high granular fractions (figure 5D). Unfortunately, we were unable to reliably determine intra-cellular pro-IL-1 levels. Overall, increased granularity, and as such intracellular lipids, did only mildly affect IL-1 $\beta$ levels in Ly6C monocytes, suggesting that monocyte lipid ingestion is not or only marginally associated with inflammasome activation.

\section{Hypercholesterolemia increases monocyte-endothelial cell interactions}

Increased plasma cholesterol levels were shown to induce inflammatory cytokine levels already after 1 week of WTD feeding. Further, ROS have been implicated to increase monocyte-endothelial cell adhesion in vitro ${ }^{33,34}$. To address the combined effect of lipid accumulation, pro-inflammatory milieu, and ROS production on monocyte function we performed in vivo monocyteendothelium adhesion experiments. At baseline, no $\mathrm{CD} 115^{+}$cells adhered to the endothelium of the vessels in the sternohyoid muscle in either mice on normal chow or 1 week WTD (data not shown).

After activation of the local vasculature with $50 \mu \mathrm{L}$ LPS $(100 \mathrm{ng} / \mathrm{mL})$ a clear increase in rolling and adhering $\mathrm{CD} 115^{+}$monocytes could be observed compared to baseline condition, where only an occasional rolling or adhering cells could be observed. $\mathrm{CD} 115^{+}$cells were considered rolling if their speed was reduced by at least two-fold and showed direct contact to the endothelial layer, adhering cells did not change position for at least 280 frames (30 seconds). Due to the small number of animals ( $n=3$ per group) examined no statistical analyses were performed. The increase in monocyte-endothelial cell interaction was most pronounced in animals on WTD. Animals on WTD showed increased adhering (figure 6A) and rolling $\mathrm{CD} 115^{+}$cells (figure 6B). Compared to baseline both animals on normal chow and WTD displayed more adhering and rolling cells, yet this increment was more pronounced in animals on WTD (figure 6C). 
A

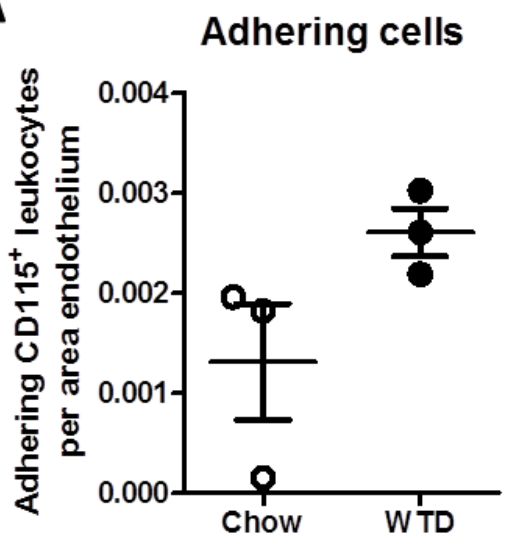

B

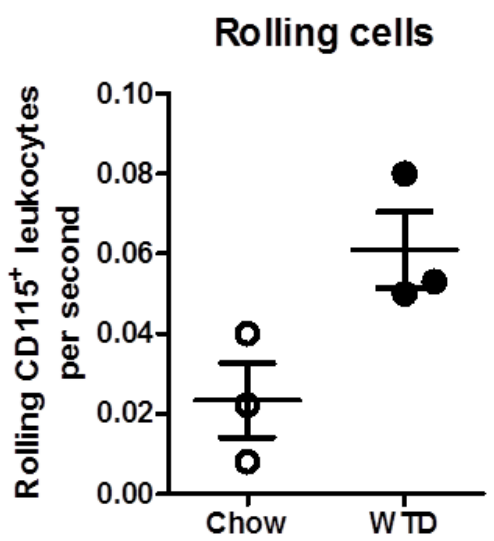

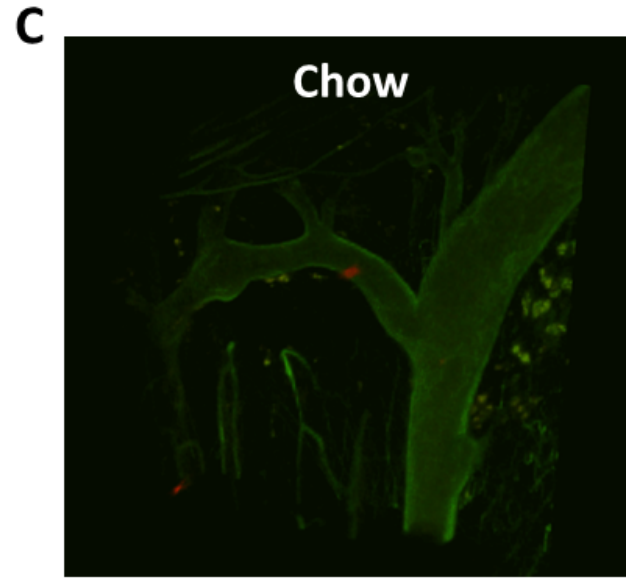

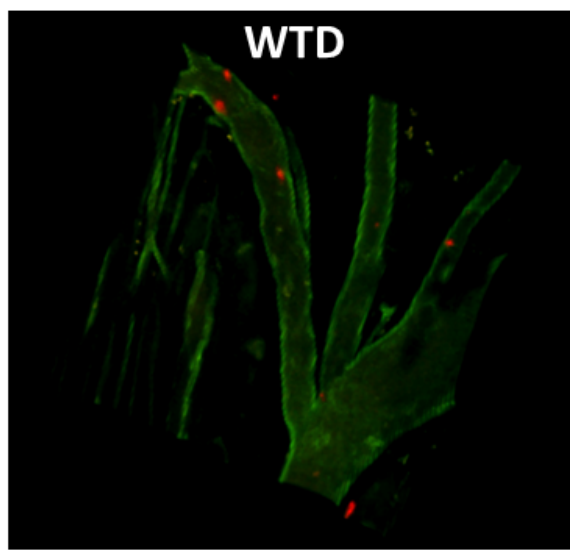

Figure 6 - In vivo monocyte-endothelial cell interaction is stimulated by intracellular cholesterol accumulation

Monocyte interaction with the endothelial layer for $\mathrm{LDLr}^{-/}$animals was measured in vivo in the sternohyoid muscle after activating the endothelium with $100 \mathrm{ng} / \mathrm{mL}$ LPS. (A) Number of steadily adhering cells ( $>30$ seconds) was determined and corrected for the area of vascular endothelium examined. (B) Number of rolling cells along the vascular endothelium was determined and corrected for the total time of imaging. (C) Representative 3D reconstructions of the vasculature for animals on normal chow (top panel) and WTD (bottom panel) are shown. Endothelial cells are labeled with anti-CD31-FITC (green) and monocytes are labeled with antiCD115-Alexa568 (red). 


\section{Discussion}

The role of circulating monocytes in the development of atherosclerosis has been extensively studied over the last few years. Monocyte subsets have been reported to play differential roles in atherogenesis. Ly $6 \mathrm{C}^{\text {high }}$ monocytes are widely considered to be the most important progenitor of plaque macrophages ${ }^{10,35}$, while Ly6C monocytes are attributed mainly vascular patrolling functions and are thought to give rise to $\mathrm{CD} 11 \mathrm{c}^{+}$dendritic cells in the lesion ${ }^{36-38}$. Recently, Wu et al have demonstrated that cholesterol can induce CD11c expression in monocytes, and enhance their adhesion to the endothelium ${ }^{39}$. This might implicate a more important role for Ly6C monocytes in atherogenesis. In this study we show that monocytes, both Ly $6 C^{\text {high }}$ and $L y 6 C$, respond differentially to the hypercholesterolemia induction.

We demonstrate that circulating, next to splenic and bone marrow, monocytes rapidly accumulate lipids under hypercholesterolemic conditions, before entering the atherosclerotic lesion, an effect accompanied by generally transient elevation in plasma cytokine levels. Moreover we show that hypercholesterolemia not only induces ROS buildup, but also upregulates IL-1 $\beta$ production by Ly $6 \mathrm{C}^{\text {high }}$ and Ly $6 \mathrm{C}^{-}$monocytes, the latter likely without activating the inflammasome pathway. The progressive accumulation of cholesterol in these peripheral monocytes could also induce the pro-inflammatory milieu observed during atherogenesis, however additional studies are necessary to support this notion. We do observe increased interaction of circulating monocytes with (activated) endothelium under hypercholesterolemic conditions.

The initial observation of progressively increased side scattering of circulating monocyte subsets, and in particular the Ly6C subset, upon WTD feeding of $\mathrm{LLr}^{-1}$ mice, has led us to investigate the cause of increased granularity in more detail. Here, we present three lines of evidence that increased granularity reflects monocytic lipid accumulation: (I) plasma cholesterol correlated strongly to the increased granularity of monocytes; (II) electron microscopy showed the overt presence of intracellular vacuoles; and (III) Oil-red-O staining established the presence of intracellular lipids in monocytes from WTD-fed $L D L r^{-1}$ mice. Interestingly, this effect was more pronounced in ly6 $\mathrm{C}^{-}$monocytes, however the responsible mechanism needs to be determined. As monocyte development is thought to develop from Ly $6 \mathrm{C}^{\text {high }}$ 
towards Ly6 $\mathrm{C}^{-}$monocytes ${ }^{40}$, the turnover from lipid-laden Ly6C $\mathrm{C}^{\text {high }}$ to Ly6C monocytes could add to the more distinct lipid accumulation in the downstream Ly6C monocyte pool. On the other hand, we cannot exclude that passive or active cholesterol/lipid accumulation, but also difference in efflux, between monocyte subsets contribute to the differences observed.

Lipid accumulation in circulating monocytes appears to have many similarities to scavenging of (ox)LDL by plaque macrophages. As lipid accumulation in macrophages activates the inflammasome, due to intracellular cholesterol crystals ${ }^{20}$, the question arose whether lipid ingestion by circulating monocytes would in analogy to macrophages also here result in inflammasome activation. Despite the presence of cholesterol crystals in circulating monocytes and the transient increase of plasma IL-1 $\beta$ levels upon diet change, involvement of the inflammasome in circulating monocytes could not be confirmed conclusively. Preliminary data supported that the IL-1 $\beta$ effects were inflammasome independent, as pro-IL-1 levels seemed to increase, reflective of transcriptional induction rather than inflammasome activation. This was reinforced by unaltered caspase 1 activity in these monocyte subsets (data not shown). Nevertheless, inflammasome-independent IL-1 $\beta$ has been implicated to be essential for sustaining extra-medullar monocytopoiesis ${ }^{41}$.

Reactive oxygen species did increase significantly in high granular monocytes carrying intracellular cholesterol deposits. While in macrophages cholesterol accumulation has already been linked to ROS production, only one single study has investigated cholesterol-dependent ROS production in monocytes ${ }^{42}$. Although the actual molecular pathway linking lipid to ROS accumulation in monocytes remains to be determined, as shown by Huang et $a /$ indicated that in humans ROS production might be mediated via C-reactive protein expression and the subsequent upregulation of NADPH oxidase (NOX) 2 and MCP-1 levels ${ }^{33}$. As increased NOX2 and MCP-1 levels might affect chemotaxis of monocytes, hypercholesterolemia induced ROS production could well have effects on the migratory behavior towards activated endothelium.

Indeed our intravital microscopy studies showed that monocyte adhesion to and rolling along activated endothelium was substantially increased in WTD fed $L D L r^{--}$mice. Apart from a ROS associated phenomenon, the increased endothelial cell adhesion could also result from lipid accumulation induced upregulation of integrins on circulating monocytes 
$26,43,44$. However, as we did not determine a broad set of membrane markers, such as ICAM-1, very late antigen (VLA) 4, and CD62L, on these circulatory monocytes, the actual contribution of this pathway to the increased endothelial adhesion remains unclear. Nevertheless, our study clearly shows that hyperlipidemia impacts not only circulating monocyte constitution but also function, already within 1 week of WTD exposure.

To what extent this is relevant to for early atherogenesis is yet to be determined, given that our in vivo studies involving LPS activated small vessels rather than major arteries. However, since endothelial cells covering the (early) atherosclerotic lesion do express TLRs ${ }^{45,46}$, the LPS stimulation used in our experiments is representative for atherosclerotic endothelium. Especially since TLR4 polymorphisms as well as MyD88 deficiency have been shown to decrease atherogenesis 47,48. Therefore, we expect cholesterol-loaden monocytes to adhere as efficiently to the endothelium covering the atherosclerotic plaques under hypercholesterolemic conditions. Atherosclerosis development might be further stimulated by ROS generation in circulating monocytes, as this contributes to the formation of oxLDL ${ }^{42}$.

Indeed monocytes in compartments relevant for myeloipoiesis, such as spleen and bone marrow, also showed pronounced lipid accumulation, although at present we cannot exclude that these monocytes had compartimentalized upon lipid ingestion or whether they ingestion had occurred after monocyte grafting in these tissues ${ }^{10,36,41}$. Nagareddy et al recently showed that monocytosis might in part result from hyperglycemia, next to hypercholesterolemia ${ }^{49}$. However, this was mediated via increased proliferation and expansion of granulocyte/macrophage progenitors in the bone marrow, which we did not observe in our study.

Monocyte development is often considered to be a linear process, inflammatory (Ly6C $\mathrm{C}^{\text {high }}$ ) monocytes produced in the bone marrow migrate towards the spleen and at least a subset will maturate towards patrolling (Ly6C $)^{-}$monocytes ${ }^{10}$. In atherosclerosis Ly6 $\mathrm{C}^{\text {high }}$ monocytes appear to be most critical as precursor for plaque macrophages ${ }^{35}$, however the role of Ly6C monocytes is still debated. In human atherosclerosis, the role of inflammatory CD14 ${ }^{\text {high }} \mathrm{CD} 16^{-}$monocytes and patrolling $\mathrm{CD} 14^{-} \mathrm{CD} 16^{+}$monocytes is even less defined, as no trafficking studies have been performed in human disease. Nevertheless, especially patrolling Ly6C monocytes show massive lipid accumulation, suggesting an important role in hypercholesterolemia and 
possibly a role in atherosclerosis development, which is more important than currently thought.

Downregulation of the cholesterol transporters ABCA1 and NiemannPick disease type $C$ protein 1 (NPC1) was recently identified as the central actor responsible for lipid accumulation in human monocytes in $\mathrm{FH}$ patients, with manifest hypercholesterolemia ${ }^{50}$ and in familial coronary artery disease patients ${ }^{51}$. ABCA1/G1 deficiency results in hypercholesterolemia-associated monocytosis 52 , however $A B C A 1 / G 1$ deficiency also results in lipid accumulation in macrophages ${ }^{53}$, and potentially circulating monocytes. Not only accumulation of lipoprotein fractions in circulating monocytes have been observed before, but also accumulation of fatty acids ${ }^{54}$, in our study we have not yet discriminated between these subsets. In contrast to Swirski et $a l^{35}$, the current study has shown that Ly6C monocytosis might be an additional process contributing to atherosclerosis, at least in $L D L r^{-/}$mice.

In conclusion, we show that hypercholesterolemia not only results in a rapid, but transient pro-inflammatory adaptive response in plasma. Furthermore, almost immediate intracellular changes in circulating as well as tissue resident monocyte subsets, including lipid accumulation and ROS production, which functionally translate in enhanced monocyte adhesion to activated endothelium. Altogether, our study reveal a novel pathway through which circulating monocytes contribute to atherosclerosis not only by fostering the subendothelial macrophage invasion but also potentially by conveying cholesterol into the atherosclerotic lesion. 


\section{Supplemental data}

Supplemental table 1 - Overview of FACS antibodies used for these studies

\begin{tabular}{|c|c|c|c|}
\hline Antibody & Conjugate & Company & Tissue \\
\hline $\mathrm{CD} 3 \varepsilon$ & eFluor450 & eBioscience & Blood \\
\hline $\mathrm{CD} 3 \varepsilon$ & PerCP-Сy5.5 & BD & Bone marrow \\
\hline CD4 & APC-H7 & $\mathrm{BD}$ & Blood, bone marrow \\
\hline CD8 $\alpha$ & FITC & eBioscience & Blood \\
\hline $\operatorname{CD} 8 \alpha$ & eFluor450 & eBioscience & Bone marrow \\
\hline $\operatorname{CD} 8 \alpha$ & APC-H7 & $\mathrm{BD}$ & Spleen \\
\hline CD11b & PE-Cy7 & BD & Blood, spleen \\
\hline CD11b & BV510 & $\mathrm{BD}$ & Bone marrow \\
\hline CD11b & eFluor450 & eBioscience & Spleen \\
\hline CD11c & PE-Cy7 & $\mathrm{BD}$ & Bone marrow, spleen \\
\hline CD45 & PerCP & Biolegend & Blood \\
\hline CD45RA (B220) & PerCP & $\mathrm{BD}$ & Bone marrow \\
\hline CD45RA (B220) & V500 & BD & Blood, spleen \\
\hline CD115 & PE & BD & Bone marrow \\
\hline CD317 (PDCA-1) & $\mathrm{PE}$ & $\mathrm{BD}$ & Spleen \\
\hline Ly6C & APC & Miltenyi & Blood, spleen, bone marrow \\
\hline Ly6G & APC-Cy7 & $\mathrm{BD}$ & Blood, spleen, bone marrow \\
\hline MHC II & FITC & BD & Bone marrow, spleen \\
\hline NK1.1 & $\mathrm{PE}$ & BD & Blood, spleen \\
\hline NK1.1 & PerCP-Сy5.5 & BD & Bone marrow \\
\hline
\end{tabular}


A

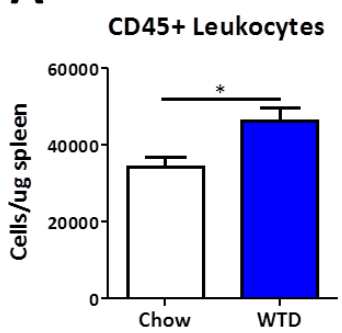

D

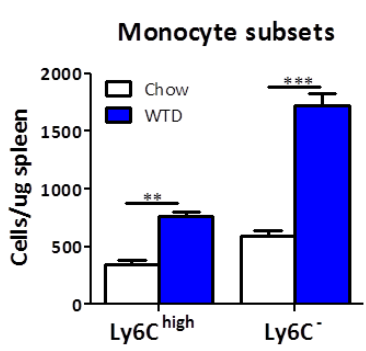

B

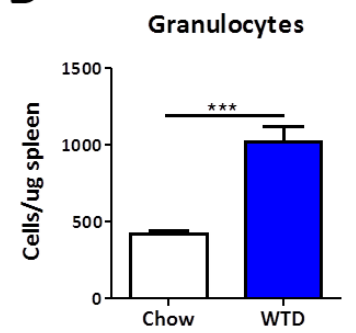

$\mathbf{E}$

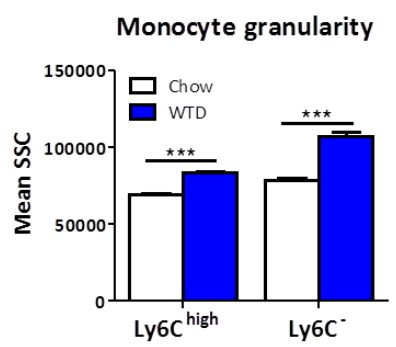

C

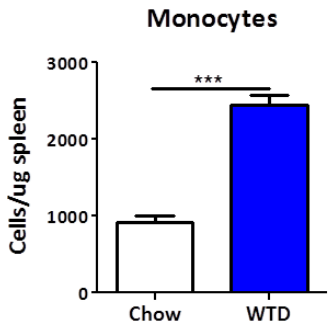

Supplemental figure 1 - Splenic leukocyte subsets increase upon WTD comparable to circulating leukocytes

Absolute leukocyte levels were measured in spleen upon sacrifice using BD TruCount tubes. (A) Total CD45+ leukocytes, (B) CD11c+ CDC, (C) pDCA1+ pDC, (D) CD11b+/Ly6G+ granulocytes, (E) CD11b+/Ly6G- monocytes, and (F) Ly6C monocyte subsets, and (G) monocyte granularity per subset were measured. ${ }^{*} p<0.05, * * p<0.01$, and ${ }^{* * *} p<0.001$ 
A

Granulocytes

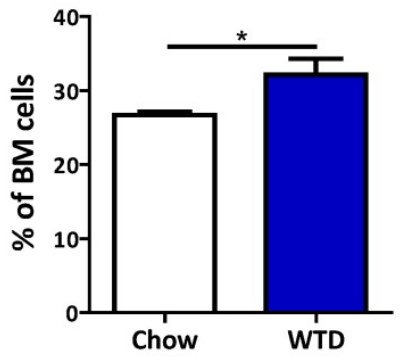

C

Monocyte subsets

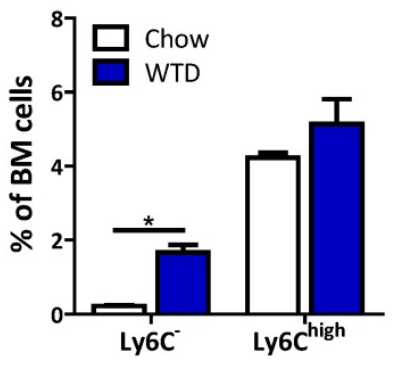

E

\section{LSK/LK/LS}

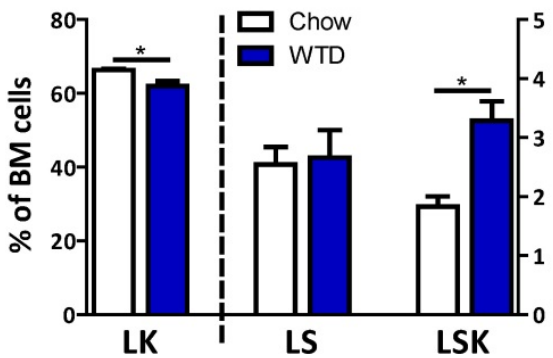

B

Monocytes

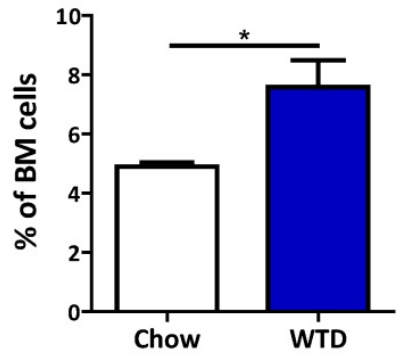

D

\section{Monocyte Granularity}

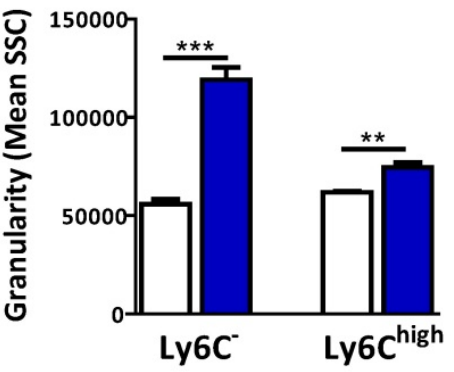

$\mathbf{F}$

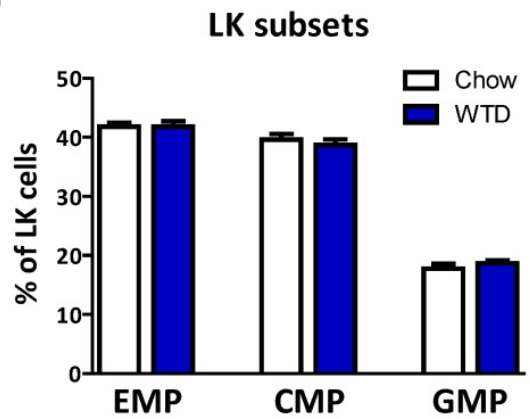

Supplemental figure 2 - Bone marrow leukocyte subsets increase upon WTD comparable to circulating leukocytes

Relative leukocyte levels were measured in bone marrow after 3 weeks of normal chow or WTD. (A) CD11b+/Ly6G+ granulocytes, (B) CD11b+/Ly6G- monocytes, (C) monocyte subsets, (D) granularity monocyte subsets, (E) progenitor cell population, and (F) LK subsets were measured. $* p<0.05, * * p<0.01$, and ${ }^{* * *} p<0.001$ 


\section{References}

1. Wilson PW, D'Agostino RB, Levy D, Belanger AM, Silbershatz $H$, Kannel WB. Prediction of coronary heart disease using risk factor categories. Circulation. 1998;97(18):1837-1847.

2. Jansen AC, van Aalst-Cohen ES, Tanck MW, et al. The contribution of classical risk factors to cardiovascular disease in familial hypercholesterolaemia: data in 2400 patients. J Intern Med. 2004;256(6):482-490.

3. Nissen SE, Tuzcu EM, Schoenhagen P, et al. Statin therapy, LDL cholesterol, Creactive protein, and coronary artery disease. N Engl J Med. 2005;352(1):29-38.

4. Ziegler-Heitbrock L, Ancuta P, Crowe $S$, et al. Nomenclature of monocytes and dendritic cells in blood. Blood. 2010;116(16):e74-80.

5. Auffray C, Fogg D, Garfa M, et al. Monitoring of blood vessels and tissues by a population of monocytes with patrolling behavior. Science. 2007;317(5838):666-670.

6. Sunderkotter C, Nikolic T, Dillon MJ, et al. Subpopulations of mouse blood monocytes differ in maturation stage and inflammatory response. J Immunol. 2004;172(7):4410-4417.

7. Zlotnik A, Yoshie O. Chemokines: a new classification system and their role in immunity. Immunity. 2000;12(2):121-127.

8. Geissmann F, Jung S, Littman DR. Blood monocytes consist of two principal subsets with distinct migratory properties. Immunity. 2003;19(1):71-82.

9. Woollard KJ, Geissmann F. Monocytes in atherosclerosis: subsets and functions. Nat Rev Cardiol. 2010;7(2):77-86.

10. Robbins CS, Chudnovskiy A, Rauch PJ, et al. Extramedullary hematopoiesis generates Ly- $6 \mathrm{C}$ (high) monocytes that infiltrate atherosclerotic lesions. Circulation. 2012;125(2):364-374.

11. Barlic J, Zhang Y, Foley JF, Murphy PM. Oxidized lipid-driven chemokine receptor switch, CCR2 to CX3CR1, mediates adhesion of human macrophages to coronary artery smooth muscle cells through a peroxisome proliferator-activated receptor gamma-dependent pathway. Circulation. 2006;114(8):807-819.

12. Manning-Tobin JJ, Moore KJ, Seimon TA, et al. Loss of SR-A and CD36 activity reduces atherosclerotic lesion complexity without abrogating foam cell formation in hyperlipidemic mice. Arterioscler Thromb Vasc Biol. 2009;29(1):19-26.

13. Greaves DR, Gordon S. The macrophage scavenger receptor at 30 years of age: current knowledge and future challenges. J Lipid Res. 2009;50 Suppl:S282-286.

14. Yvan-Charvet L, Ranalletta M, Wang $N$, et al. Combined deficiency of ABCA1 and $A B C G 1$ promotes foam cell accumulation and accelerates atherosclerosis in mice. $J$ Clin Invest. 2007;117(12):3900-3908.

15. Westerterp M, Murphy AJ, Wang M, et al. Deficiency of ATP-Binding Cassette Transporters $A 1$ and $\mathrm{G} 1$ in Macrophages Increases Inflammation and Accelerates Atherosclerosis in Mice. Circ Res. 2013;112(11):1456-1465.

16. Stewart CR, Stuart LM, Wilkinson K, et al. CD36 ligands promote sterile inflammation through assembly of a Toll-like receptor 4 and 6 heterodimer. Nat Immunol. 2010;11(2):155-161. 
17. Seimon TA, Nadolski MJ, Liao X, et al. Atherogenic lipids and lipoproteins trigger CD36-TLR2-dependent apoptosis in macrophages undergoing endoplasmic reticulum stress. Cell Metab. 2010;12(5):467-482.

18. Li LC, Varghese Z, Moorhead JF, Lee CT, Chen JB, Ruan XZ. Cross-talk between TLR4-MyD88-NF-kappaB and SCAP-SREBP2 pathways mediates macrophage foam cell formation. Am J Physiol Heart Circ Physiol. 2013;304(6):H874-884.

19. Weber C, Noels H. Atherosclerosis: current pathogenesis and therapeutic options. Nat Med. 2011;17(11):1410-1422.

20. Rajamaki K, Lappalainen J, Oorni K, et al. Cholesterol crystals activate the NLRP3 inflammasome in human macrophages: a novel link between cholesterol metabolism and inflammation. PLoS One. 2010;5(7):e11765.

21. Duewell $\mathrm{P}$, Kono $\mathrm{H}$, Rayner KJ, et al. NLRP3 inflammasomes are required for atherogenesis and activated by cholesterol crystals. Nature. 2010;464(7293):13571361.

22. Fotis L, Agrogiannis G, Vlachos IS, et al. Intercellular adhesion molecule (ICAM)-1 and vascular cell adhesion molecule (VCAM)-1 at the early stages of atherosclerosis in a rat model. In Vivo. 2012;26(2):243-250.

23. Sun C, Alkhoury K, Wang YI, et al. IRF-1 and miRNA126 modulate VCAM-1 expression in response to a high-fat meal. Circ Res. 2012;111(8):1054-1064.

24. Zhou Z, Subramanian P, Sevilmis G, et al. Lipoprotein-derived lysophosphatidic acid promotes atherosclerosis by releasing CXCL1 from the endothelium. Cell Metab. 2011;13(5):592-600.

25. Martinovic I, Abegunewardene N, Seul M, et al. Elevated monocyte chemoattractant protein-1 serum levels in patients at risk for coronary artery disease. Circ J. 2005;69(12):1484-1489.

26. Fu C, He J, Li C, Shyy JY, Zhu Y. Cholesterol increases adhesion of monocytes to endothelium by moving adhesion molecules out of caveolae. Biochim Biophys Acta. 2010;1801(7):702-710.

27. Galkina E, Ley K. Immune and inflammatory mechanisms of atherosclerosis (*). Annu Rev Immunol. 2009;27:165-197.

28. Tolani S, Pagler TA, Murphy AJ, et al. Hypercholesterolemia and reduced HDL$\mathrm{C}$ promote hematopoietic stem cell proliferation and monocytosis: Studies in mice and FH children. Atherosclerosis. 2013.

29. Rothe G, Gabriel H, Kovacs E, et al. Peripheral blood mononuclear phagocyte subpopulations as cellular markers in hypercholesterolemia. Arterioscler Thromb Vasc Biol. 1996;16(12):1437-1447.

30. Yvan-Charvet L, Pagler T, Gautier EL, et al. ATP-binding cassette transporters and HDL suppress hematopoietic stem cell proliferation. Science. 2010;328(5986):1689-1693.

31. Tall AR, Yvan-Charvet L, Westerterp M, Murphy AJ. Cholesterol efflux: a novel regulator of myelopoiesis and atherogenesis. Arterioscler Thromb Vasc Biol. 2012;32(11):2547-2552.

32. van Bussel BC, Ferreira I, van de Waarenburg MP, et al. Multiple inflammatory biomarker detection in a prospective cohort study: a cross-validation between wellestablished single-biomarker techniques and an electrochemiluminescense-based multi-array platform. PLoS One. 2013;8(3):e58576. 
33. Huang $\mathrm{X}$, Zhang J, Liu J, et al. C-reactive protein promotes adhesion of monocytes to endothelial cells via NADPH oxidase-mediated oxidative stress. J Cell Biochem. 2012;113(3):857-867.

34. Chuang KP, Huang YF, Hsu YL, Liu HS, Chen HC, Shieh CC. Ligation of lymphocyte function-associated antigen-1 on monocytes decreases very late antigen4-mediated adhesion through a reactive oxygen species-dependent pathway. Blood. 2004;104(13):4046-4053.

35. Swirski FK, Libby P, Aikawa E, et al. Ly-6Chi monocytes dominate hypercholesterolemia-associated monocytosis and give rise to macrophages in atheromata. J Clin Invest. 2007;117(1):195-205.

36. Hilgendorf I, Swirski FK. Making a difference: monocyte heterogeneity in cardiovascular disease. Curr Atheroscler Rep. 2012;14(5):450-459.

37. Tacke F, Alvarez D, Kaplan TJ, et al. Monocyte subsets differentially employ CCR2, CCR5, and CX3CR1 to accumulate within atherosclerotic plaques. J Clin Invest. 2007;117(1):185-194.

38. Carlin LM, Stamatiades EG, Auffray C, et al. Nr4a1-dependent Ly6C(low) monocytes monitor endothelial cells and orchestrate their disposal. Cell. 2013;153(2):362-375.

39. Wu H, Gower RM, Wang $\mathrm{H}$, et al. Functional role of CD11c+ monocytes in atherogenesis associated with hypercholesterolemia. Circulation. 2009;119(20):27082717.

40. Swirski FK. The spatial and developmental relationships in the macrophage family. Arterioscler Thromb Vasc Biol. 2011;31(7):1517-1522.

41. Leuschner F, Rauch PJ, Ueno $T$, et al. Rapid monocyte kinetics in acute myocardial infarction are sustained by extramedullary monocytopoiesis. J Exp Med. 2012;209(1):123-137.

42. Vasconcelos EM, Degasperi GR, de Oliveira HC, Vercesi AE, de Faria EC, Castilho LN. Reactive oxygen species generation in peripheral blood monocytes and oxidized LDL are increased in hyperlipidemic patients. Clin Biochem. 2009;42(12):12221227.

43. Gaudreault N, Kumar N, Posada JM, et al. ApoE suppresses atherosclerosis by reducing lipid accumulation in circulating monocytes and the expression of inflammatory molecules on monocytes and vascular endothelium. Arterioscler Thromb Vasc Biol. 2012;32(2):264-272.

44. Chen CY, Yi L, Jin X, et al. Inhibitory effect of delphinidin on monocyteendothelial cell adhesion induced by oxidized low-density lipoprotein via ROS/p38MAPK/NF-kappaB pathway. Cell Biochem Biophys. 2011;61(2):337-348.

45. Frantz S, Kobzik L, Kim YD, et al. Toll4 (TLR4) expression in cardiac myocytes in normal and failing myocardium. J Clin Invest. 1999;104(3):271-280.

46. Edfeldt K, Swedenborg J, Hansson GK, Yan ZQ. Expression of toll-like receptors in human atherosclerotic lesions: a possible pathway for plaque activation. Circulation. 2002;105(10):1158-1161.

47. Kiechl S, Lorenz E, Reindl M, et al. Toll-like receptor 4 polymorphisms and atherogenesis. N Engl J Med. 2002;347(3):185-192. 
48. Michelsen KS, Wong MH, Shah PK, et al. Lack of Toll-like receptor 4 or myeloid differentiation factor 88 reduces atherosclerosis and alters plaque phenotype in mice deficient in apolipoprotein E. Proc Natl Acad Sci U S A. 2004;101(29):10679-10684.

49. Nagareddy PR, Murphy AJ, Stirzaker RA, et al. Hyperglycemia promotes myelopoiesis and impairs the resolution of atherosclerosis. Cell Metab. 2013;17(5):695-708.

50. Mosig S, Rennert K, Buttner P, et al. Monocytes of patients with familial hypercholesterolemia show alterations in cholesterol metabolism. BMC Med Genomics. 2008;1:60.

51. Sivapalaratnam S, Basart H, Watkins NA, et al. Monocyte gene expression signature of patients with early onset coronary artery disease. PLoS One. 2012;7(2):e32166.

52. Westerterp M, Gourion-Arsiquaud S, Murphy AJ, et al. Regulation of hematopoietic stem and progenitor cell mobilization by cholesterol efflux pathways. Cell Stem Cell. 2012;11(2):195-206.

53. Mauldin JP, Nagelin MH, Wojcik AJ, et al. Reduced expression of ATP-binding cassette transporter $\mathrm{G} 1$ increases cholesterol accumulation in macrophages of patients with type 2 diabetes mellitus. Circulation. 2008;117(21):2785-2792.

54. den Hartigh LJ, Connolly-Rohrbach JE, Fore S, Huser TR, Rutledge JC. Fatty acids from very low-density lipoprotein lipolysis products induce lipid droplet accumulation in human monocytes. J Immunol. 2010;184(7):3927-3936. 
Hypercholesterolemia triggers monocyte lipoprotein accumulation 



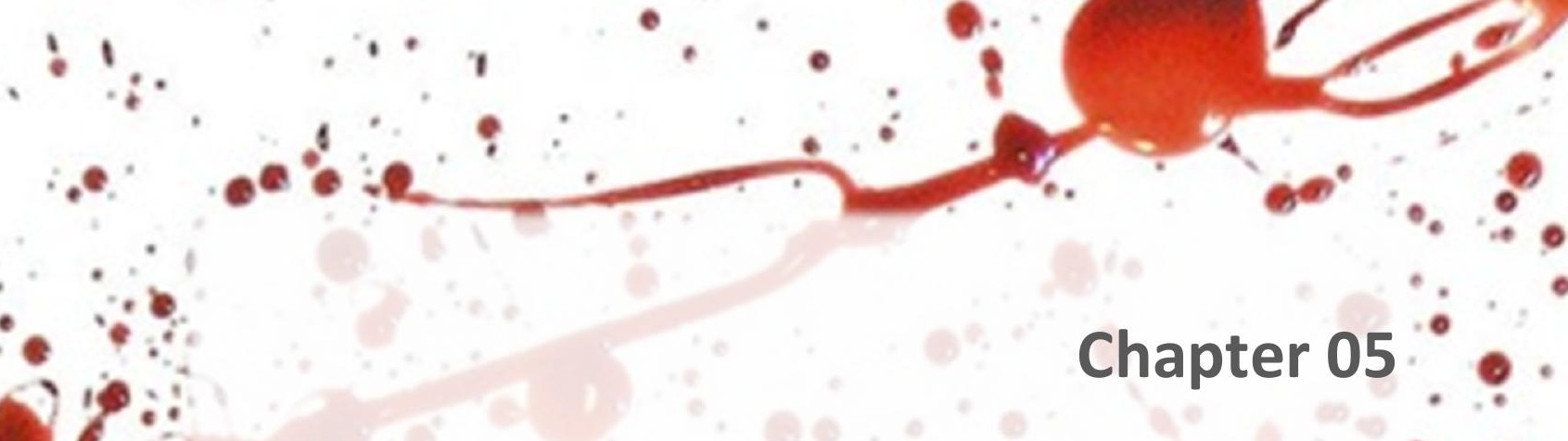

Phage display identifies novel,$\because$ circulating and plaque monocyte ${ }^{8} \cdot$ markers for advanced

\section{atherosclerosis}

Jeroen JT Otten, Nynke van den Akker, Mat JM Rousch, Ivo Eijkenboom, Fabienne Jeukens, Mick Gagliardi, J Wouter Jukema, C Theo Verrips, 8 * Imo E Hoefer, Johannes Waltenberger, Mohamed El-Khattabi, Erik AL Biessen

Submitted

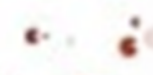

148
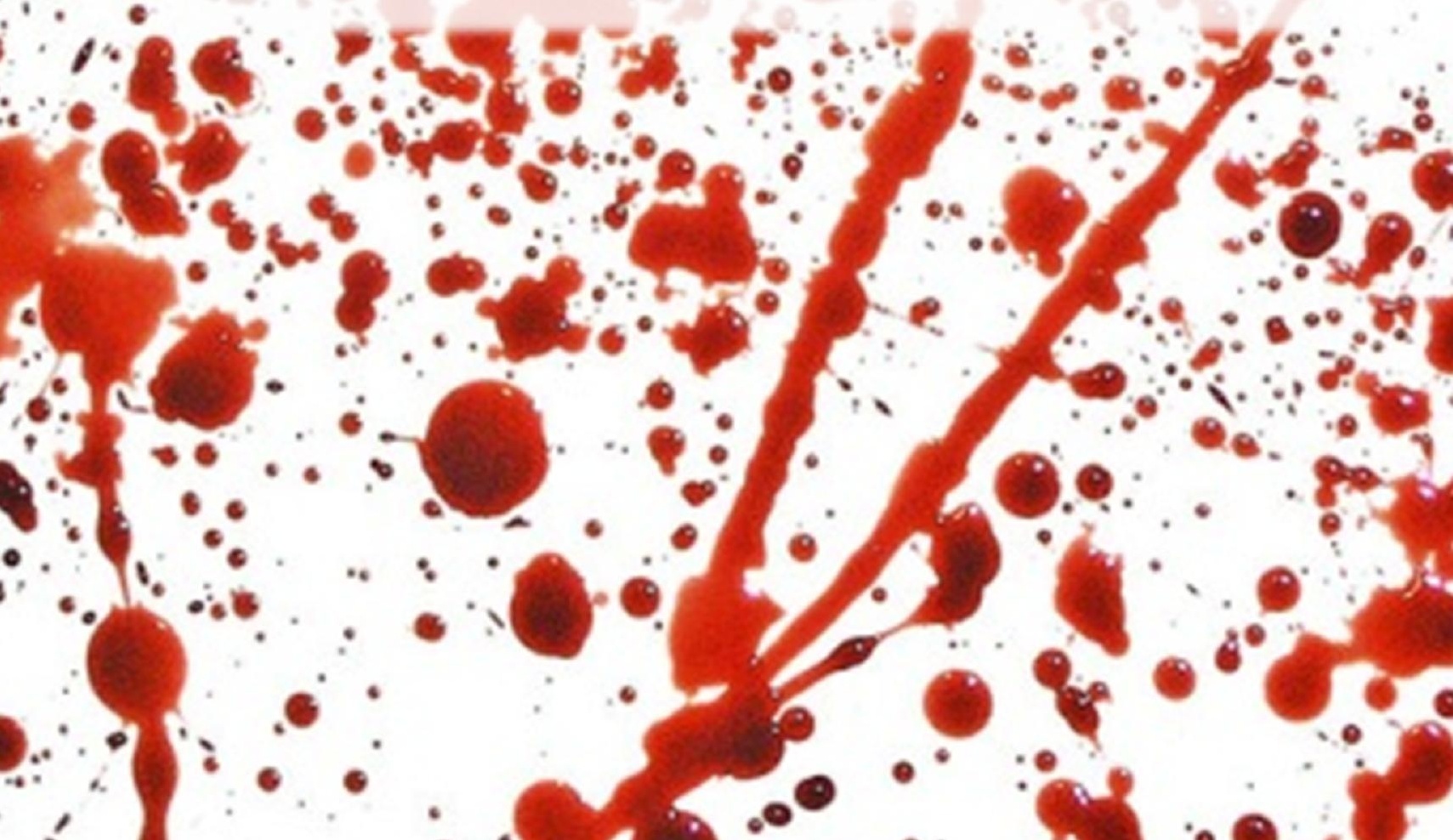


\begin{abstract}
Unstable angina pectoris is generally diagnosed based on clinical parameters, in default of accessible, sensitive, and selective biomarkers. In this study, we sought to identify novel and unstable angina pectoris-specific biomarkers on circulating monocytes, by phage display $\mathrm{V}_{\mathrm{HH}}$ antibody repertoire screening. Hereto, a dedicated antibody library was generated from plasma cells isolated from llamas, immunized with unstable angina pectoris patient monocytes. Affinity selections on human LPS-activated THP-1 monocytes, afforded several clones with increased affinity to LPS-activated THP-1 monocytes. Subsequent validation of selected clones on CD14 positive monocytes derived from unstable angina pectoris patients versus monocytes derived from healthy volunteers, identified clones which preferentially recognized unstable angina pectoris patient monocytes. Finally, selected clones specifically bound circulating human monocytes (ELISA) as well as monocytic cells in the vulnerable atherosclerotic lesion (immunohistochemistry). In conclusion, we have identified novel monocyte binding phage clones and provide proof of concept for their potential as biomarker in unstable angina pectoris diagnosis and in molecular imaging approaches for vulnerable plaque detection.
\end{abstract}




\section{Introduction}

Acute coronary syndromes continue to be a major cause of morbidity and mortality in the Western society ${ }^{1,2}$. The clinical manifestations generally are caused by rupture of an unstable atherosclerotic lesion ${ }^{3}$. Nowadays, there is increasing interest in the characteristics of such unstable, rupture-prone lesions, not only from a mechanistic, but also from a diagnostic point of view, as unstable plaque markers are deemed very useful for molecular imaging purposes ${ }^{4,5}$. However, many of these proposed markers are non-selective or difficult to monitor using non- or minimally invasive methods ${ }^{6}$. Growing evidence suggests that information on localized events in the vasculature or even focal processes in the vulnerable atherosclerotic lesion can probably be deduced from information accessible in the periphery ${ }^{5,7,8}$. The current study explores the possibilities of employing circulating monocytes as a read-out for diagnostic and potentially prognostic markers for unstable angina pectoris and acute, but also future, cardiovascular events.

Monocytes may be a particular relevant source for novel biomarkers, as monocyte-derived macrophages are instrumental in plaque inflammation, a major risk factor in plaque rupture ${ }^{9}$. Furthermore, membrane protein expression on circulating leukocytes, and especially monocytes, are highly responsive to external influences, such as pathogens ${ }^{10}$, trauma released factors, and inflammatory disease ${ }^{11,12}$. In response to these pathological processes foreign material can be presented on specialized cells. Cell types most famous for presenting antigens on MHC class I or II molecules are dendritic cells ${ }^{13}$ and macrophages ${ }^{14}$. Moreover, many of the differentially expressed membrane proteins, such as MHC class II, TLR4, and CD14 are associated with functional alterations or adaptations of the monocytes as they trigger or dampen the activity of downstream effectors. Overall, the modifications of surface marker expression on circulating monocytes due to local vascular events are of interest as these may harbor biomarker potential.

Leukocyte membrane markers, or the expression level of these markers, have repeatedly been shown to be affected by localized vascular events. Acute cardiovascular events was seen to result in altered receptor expression profiles on monocytes ${ }^{15,16}$. Conceivably, the $\mathrm{CD} 14^{+} \mathrm{CD} 16^{+}$subset of monocytes which have a patrolling function, would be among the first to react to vascular injury or trauma. The information on local pathophysiological processes may therefore be directly analyzed from peripherally sampled blood 
specimen, or possibly even using (non-invasive) imaging techniques. The latter could be further optimized using novel, highly specific markers for vulnerable atherosclerotic lesions. At the moment knowledge about the relation between monocyte expression profiles and cardiovascular disease are largely lacking.

We therefore opted for an unbiased approach to screen for antibodies that target membrane markers which are differentially expressed between monocytes from healthy controls and patients with unstable angina pectoris. The advantages of phage display derived $\mathrm{V}_{\mathrm{HH}}$ antibodies are the direct application of the encoded antibody for targeted monocyte-based biomarker assays or imaging approaches. Further, the ability of these antibodies to bind targets non-accessible with conventional antibodies, such as pockets, could reveal targets unknown so far. Hereto, phage display was used as a tool to determine differences between monocyte populations, without any a priori knowledge. Normally, phage display is used to obtain $\left(\mathrm{V}_{\mathrm{HH}}\right)$ antibodies against known targets, preferentially purified or recombinant proteins ${ }^{17}$, as well as cellular or plasma derived (purified) proteins ${ }^{18,19}$. In this study the protocol was adapted to be able to determine novel targets specific for unstable atherosclerotic disease on circulating mononuclear cells ${ }^{20}$. Screening was performed using an immune library, produced by immunization of a llama with UAP patient-derived circulating monocytes. We now present data on phage/antibody selections on UAP CD14 $4^{+}$monocytes specific phage clones, and show that selected clones preferentially bind monocytic cells in the advanced, vulnerable lesion. The high affinity for UAP monocytes indicates prospective potential for use in the clinics as diagnostic marker in blood or for detection of vulnerable plaques by imaging. 


\section{Materials \& Methods}

\section{Subjects}

Monocytes (CD14 ${ }^{+}$fraction) isolated from seven patients admitted to the University Medical Center Maastricht with unstable angina pectoris, were included for the analysis performed. One patient was later excluded as clinical parameters indicated no aberrations the ECG, all other patients were confirmed to have unstable angina pectoris by the clinician. For an optimal signal-to-noise ratio compared to non-diseased monocytes we included CD14 ${ }^{+}$ monocytes from four healthy volunteers, without any known cardiovascular disease, as reference population. All participants provided written informed consent prior to participation. This study was approved by the local ethics committee.

\section{Monocyte Isolation}

Whole blood samples isolated from patients and healthy controls were centrifuged at $156 \mathrm{x}$ g for $15 \mathrm{~min}$ without break. Next, plasma was removed and stored for future analysis at $-80^{\circ} \mathrm{C}$. A leucosep tube (Greiner) was filled with $15 \mathrm{~mL}$ Ficoll (Sigma) at room temperature. Tubes were centrifugated for 30 seconds at $1,000 \times \mathrm{g}$ and 25 to $30 \mathrm{~mL}$ of PBS diluted blood was added on top of the filter and centrifuged for $20 \mathrm{~min}$ at 1,000 x g without breaks. Liquid above the peripheral blood mononuclear cells (PBMC) enriched interphase was removed and the PBMC fraction was transferred to a fresh $50 \mathrm{~mL}$ tube and washed twice with cell selection buffer (PBS; 0.5\% BSA, 2 mM EDTA, 0.09\% sodium azide). Cells were resuspended in $1 \mathrm{~mL}$ cell selection buffer and a $20 \mu \mathrm{L}$ aliquot was saved as reference. After centrifugation the pellet was resuspended in $75 \mu \mathrm{L}$ anti-human CD14 antibody conjugated magnetic beads (BD) and incubated for $60 \mathrm{~min}$ on ice. Subsequently, $1 \mathrm{~mL}$ cell selection buffer was added and cell suspension was placed on the BD Imagnet (BD) for $10 \mathrm{~min}$. With the tube on the magnet the CD14 negative fraction was aspirated and saved for other analyses. The CD14 positive fraction was removed from the magnet resuspended in cell selection buffer and incubated for an additional 4 min on the magnet. The positive cell fraction was resuspended in freezing medium and stored at $-80^{\circ} \mathrm{C}$ until further use. 


\section{Cell lines}

Unless stated otherwise, THP-1 monocytes, either lipopolysaccharide (LPS) stimulated or non-stimulated, were used for phage selection assays and affinity screenings. Cells were cultured in RPMI-1640 medium supplemented with glutamine and HEPES (Life Technologies) at $37^{\circ} \mathrm{C}$ and $5 \% \mathrm{CO}_{2}$. Cells were incubated for 1 hours with LPS (100 ng/mL; activated THP-1) or PBS (nonactivated THP-1), prior to the experiment, to induce cell activation and emulate the inflammatory conditions as observed during unstable angina pectoris.

\section{Phage library}

The immune library was produced by injecting a llama (Lama glama) with whole cell $\mathrm{CD} 14^{+}$monocytes from unstable angina pectoris (UAP) patients. Two llamas were immunized at weekly intervals for a period of 6 weeks with $\mathrm{CD} 14^{+}$ monocyte pools from 4 UAP patients. Peripheral blood lymphocytes (PBL) were isolated from immunized animals to create an immune $V_{H H}$ library, as previously described ${ }^{21}$. In short, PBL were isolated by Ficoll gradient and subsequently total RNA was isolated and transcribed into cDNA. Next, the repertoire of Ig heavy chain-encoding gene segments were amplified with the use of a framework 1 (FR1) specific primer and an oligo-dT primer. Finally, selected and purified cDNA fragments were ligated in a phagemid vector for display on filamentous bacteriophage ${ }^{22}$ and electrotransformed to Escherichia coli TG1 (K12, _(lac-pro), supE, thi, hsdD5/F'traD36, proA+B+, laclq, lacZ_M15). This resulted in a immune phage library with a diversity of approximately $8 \times$ $10^{7}$. The animal experiments were reviewed by the ethical committee and approved by the board of Utrecht University.

\section{Whole Cell $\mathrm{V}_{\mathrm{HH}}$ Selections}

The phage library was rescued by inoculation in 2xTY medium (supplemented with ampicillin) and grown for $75 \mathrm{~min}$ at $37^{\circ} \mathrm{C}$ and $270 \mathrm{rpm}$. Subsequently, M13-helperphage (with kanamycin selection vector) was added in 10:1 ratio and incubated another $30 \mathrm{~min}$. Cells were pelleted and grown overnight in 2xTY medium supplemented with ampicillin and kanamycin at $30^{\circ} \mathrm{C}$ and 270 rpm. Next day, phages were PEG-precipitated and blocked using $2 \%$ nonskimmed milk powder (Marvel) in PBS to prevent non-specific binding or sticking of phages to cells. Cell suspensions were also blocked for $30 \mathrm{~min}$ in 
2\%Marvel-PBS prior to selection. Blocked phages were added to control and CFSE-labeled, LPS-activated THP-1 cells (10:1 ratio), or CD14 ${ }^{+}$monocytes from healthy controls and CFSE-labeled CD14 ${ }^{+}$monocytes from UAP patients (10:1 ratio) and incubated for 30 min rotating at RT. Activated THP-1 cells or CD14 ${ }^{+}$ monocytes from UAP patients were isolated by FACS sorting using a BD FACS Aria (BD) cell sorter based on the CFSE expression. Sorted cells were resuspended in $200 \mathrm{mM}$ triethylamine (TEA; $\mathrm{pH}=12$ ), incubated for approximately $10 \mathrm{~min}$ and subsequently neutralized with an equal volume $1 \mathrm{M}$ Tris- $\mathrm{HCl}(\mathrm{pH}=6.8)$. Isolated phages were used to infect exponential TG1 $(O D=0.5)$ and masterplates and titrations of input and output titer were determined, to determine enrichment for each selection round. Masterplates were used to prepare new glycerol solution, which was used as starting library for subsequent selection round (figure 1).

\section{Whole Cell $\mathrm{V}_{\mathrm{HH}}$ Screenings}

Screenings were performed on either libraries from complete selection rounds or on individually selected clones. Marvel blocked control and LPS activated cells were incubated with $120 \mu \mathrm{L}$ phage suspension in blocked 96-wells Vshaped ELISA plates (Greiner) for 30 minutes at RT. Subsequently, $100 \mu \mathrm{L}$ of either control cells or activated cells were added to $100 \mu \mathrm{L}$ phage suspension and incubated for 60 minutes at $37^{\circ} \mathrm{C}$. Plates were washed with Marvel-PBS three times and PBS two times.

Phages bound were detected using an HRP-conjugated anti-M13 antibody which was incubated with the cells for 30 minutes at $37^{\circ} \mathrm{C}$. After three more wash steps bound phages were detected using TMB (KPL Inc., MD, USA) and analyzed in a plate reader (BioRad) at $450 \mathrm{~nm}$ (figure 1). 
1

B-cells

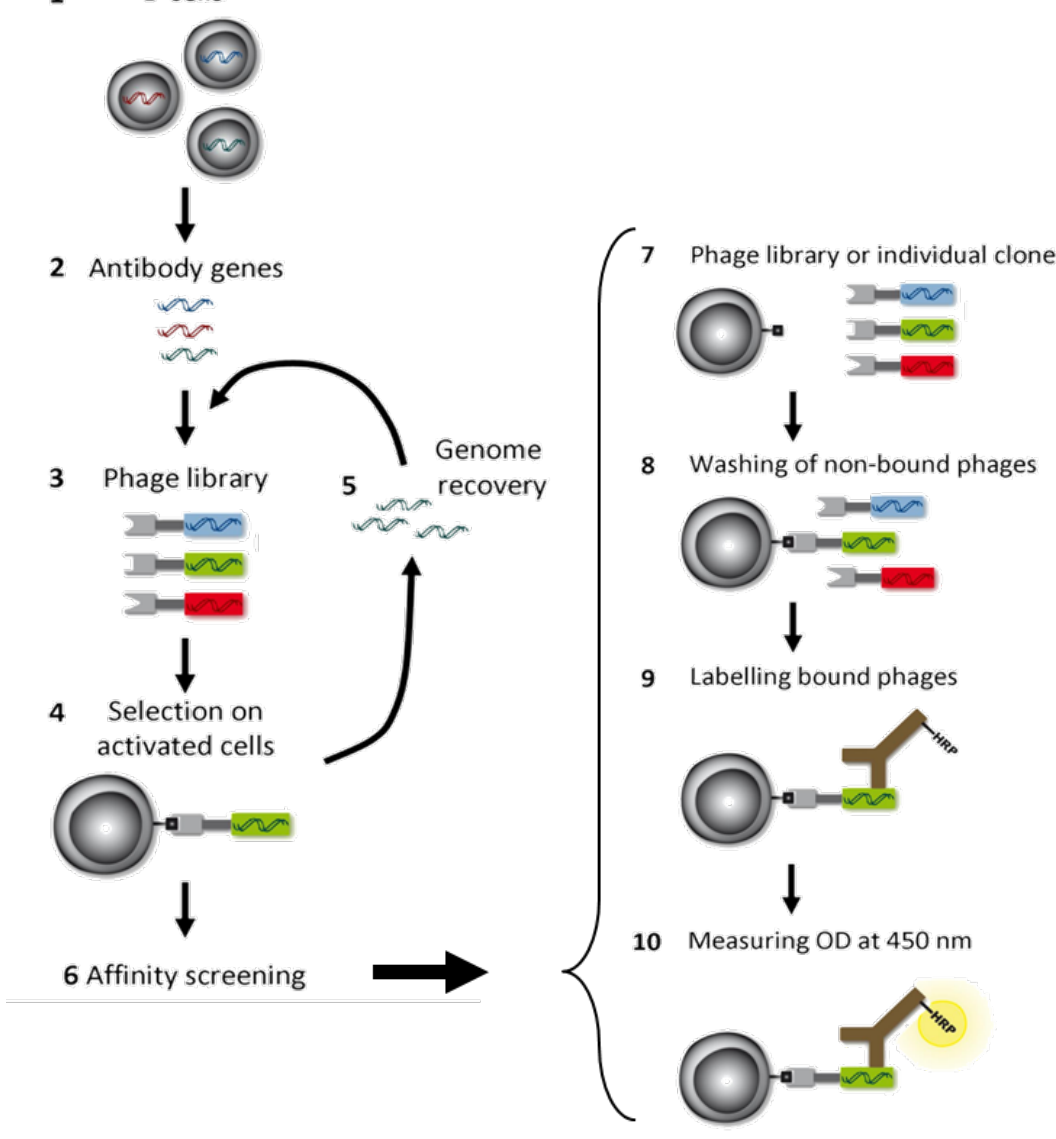

Figure 1 - Selection and screening using whole cell phage display

Phage display selections were performed according to the steps $1-5$. In short, 1 . B-cells were isolated from immunized llamas; 2 . Heavy chain $\left(\mathrm{V}_{\mathrm{HH}}\right)$ coding RNA was isolated and amplified; 3. $\mathrm{V}_{\mathrm{HH}} \mathrm{CDNA}$ was cloned/transfected into phagemid vector; 4. Selection on whole cells; 5. Recovery of selected phages. Subsequent affinity screening (step 6) was performed according to the steps $7-10$ of the procedure shown. In short, 7. production of complete library of individual clone followed by binding to whole cells; 8 . Washing to remove unbound phages; 9. Labeling of bound phages using anti-M13-HRP; 10. Detection of bound phages. Adapted in part from Hoogenboom (2005) ${ }^{17}$.

\section{Flow Cytometry}

Purity of isolated CD14 positive monocytes was analyzed using anti-CD14 PC7 and anti-CD16 PC5 (both BD). Measurements were performed on a BD Canto II flow cytometer (BD). In short, cells were pelleted and incubated with 1:25 diluted anti-CD14 PC7 and 1:25 diluted anti-CD16 PC5 antibodies for 30 min on ice in the dark. Subsequently cells were washed twice, dissolved in $200 \mu \mathrm{L}$ FACS buffer, and measured. 
CD14 positive monocytes from unstable angina pectoris patients were labeled for $15 \mathrm{~min}$ with $1 \mu \mathrm{M}$ carboxyfluorescein diacetate succinimidyl ester (CFSE; Invitrogen) in serum-free medium. Subsequently, cells were washed twice in medium supplemented with $10 \%$ fetal calf serum. Phages were added to control monocytes and CFSE-labeled UAP monocytes. After incubation for 60 minutes, monocytes from UAP patients were recovered by FACS sorting based on CFSE signal. In short, cells were spun down after incubation with the phages and washed twice in PBS-2\%Marvel and twice in PBS. Next cells were resuspended in PBS and sorted on a BD FACS Aria Cell sorter (BD). Both CFSE positive and CFSE negative fractions were isolated to determine efficiency and enrichment of the selection rounds.

\section{Immunohistochemistry}

Formalin fixed paraffin embedded sections from human carotid endarterectomy samples were deparrafinized and rehydrated. Sections were blocked for 30 minutes using 2\% Marvel-PBS. Pre-blocked phages were added at a concentration of approximately $10^{7}$ phages $/ \mathrm{mL}$ in a total volume of $150 \mu \mathrm{L}$ per section and incubated for 60 minutes at RT. Sections were washed in subsequently 2\%Marvel-PBS and PBS. Pre-blocked mouse anti-M13-HRP antibody (1:5000) was added to the sections and incubated for 30 minutes at $37^{\circ} \mathrm{C}$. Next, slides were washed in PBS and Envision anti-mouse-HRP was added for 30 minutes to amplify the signal. After an additional wash step staining was developed using $D A B$ and counterstained with haematoxylin. Finally, slides were dehydrated and coverslips were placed using Entellan.

\section{Statistical analysis}

Data are expressed as mean \pm SEM. To compare individual groups, 2-tailed Students t-test was used; non-parametric data were analyzed using MannWhitney $\mathrm{U}$ test. All analyses were performed using GraphPad Prism 5 software (GraphPad Software Inc, La Jolla, CA, USA) and p-values below 0.05 were considered statistically significant. 


\section{Results}

A B
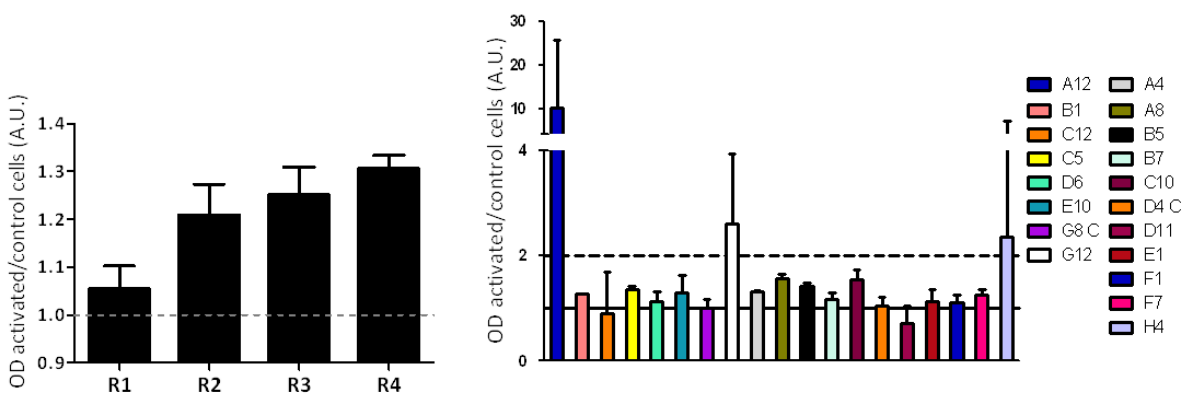

C

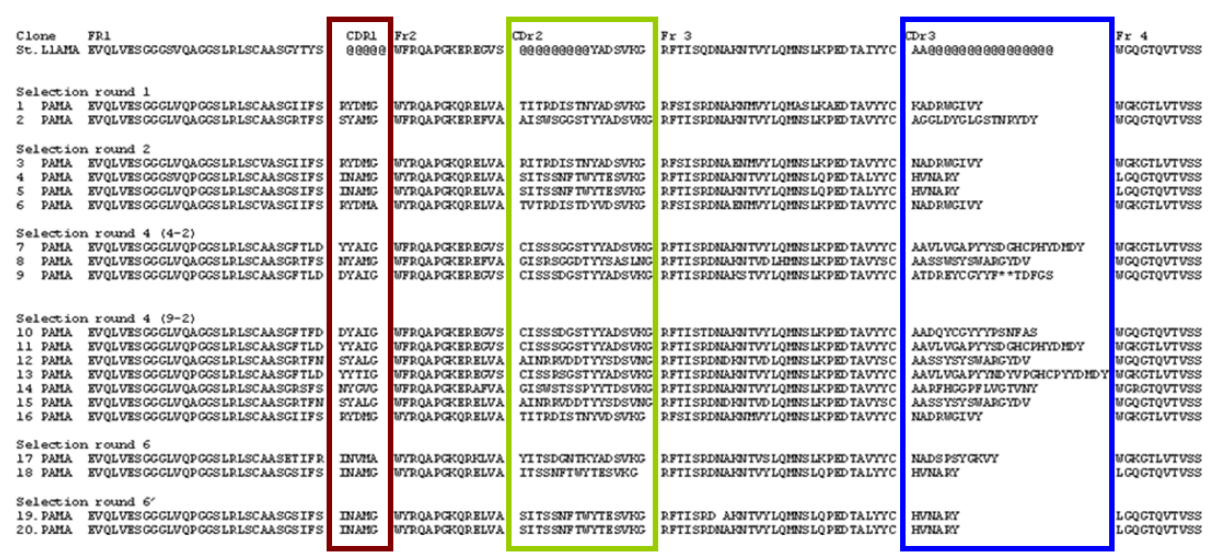

Figure 2 - THP-1 selection rounds reveal subtle yet increasing binding affinity

(A) Enrichment of the complete library after each selection round (R), indicating increased binding affinity towards activated THP-1 cells over control THP-1 cells. (B) Enrichment after selection round 4 showed heterogenous affinity to activated cells for 19 different clones selected from this selection round. Results are based on a minimum of three screenings per clone. Results are shown as mean \pm SEM, solid line indicates differential OD $=1$ and dashed line indicates differential $O D=2$. (C) Sequencing analysis of 20 clones showed variability in CDr1 (red), CDr2 (green), and mainly CDr3 (blue) region of $\mathrm{V}_{\mathrm{HH}}$ antibodies.

\section{THP-1 selection rounds revealed small number of high affinity clones}

Initial selection rounds were performed on the THP-1 human monocytic cells, which were LPS-activated to simulate the pro-inflammatory milieu in CVD patients. After 4 selection rounds we analyzed the complete phage libraries of each selection rounds (figure $2 \mathrm{~A}$ ) as well as a number of individual clones from selection round 4 (figure $2 \mathrm{~B}$ ) to assess the general enrichment in monocyte 
binding phages in the library as well as to pinpoint individual clones with highest affinity for LPS-activated monocytes. We observed a small increase in the affinity of the whole library. However, when analyzing the individual clones a large variety in affinity was observe for all selection rounds.

To determine whether the variety of clones present after the different selection rounds was affected between the different selection rounds we performed sequence analysis of the variable region for randomly selected clones from multiple selection rounds. Overall, enrichment resulted in diminished sequence variety. Still, at least some variety could be observed indicating the absence of a, possibly non-specific, dominant clone (figure $2 \mathrm{C}$ ).

A

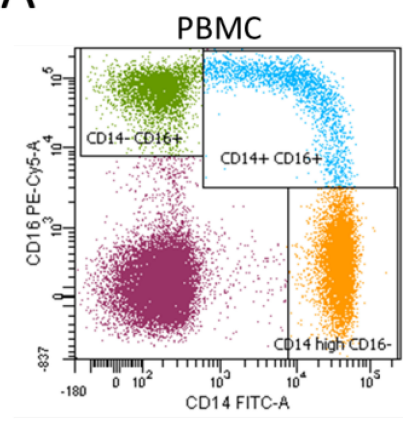

B

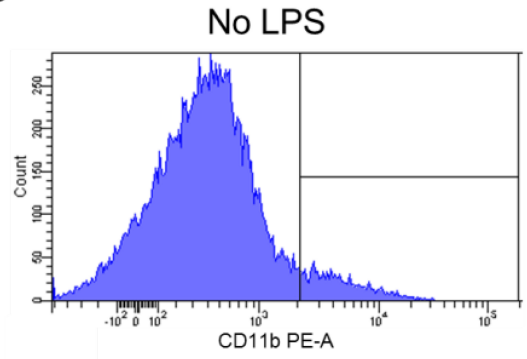

CD14+ Monocytes
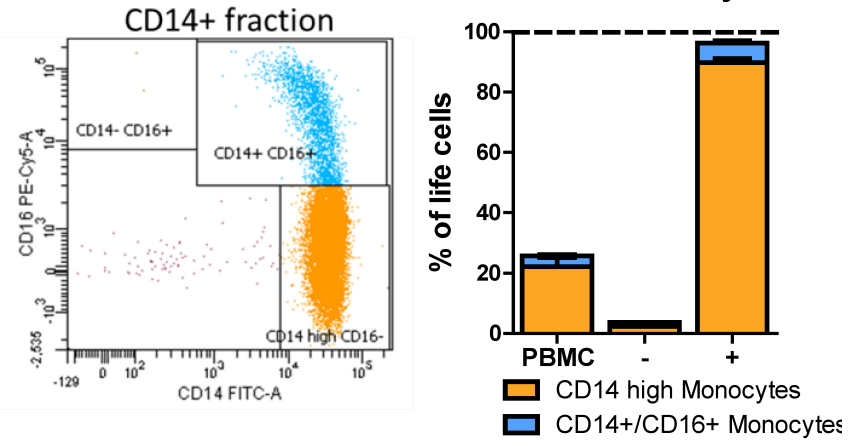

$100 \mu \mathrm{M}$ LPS

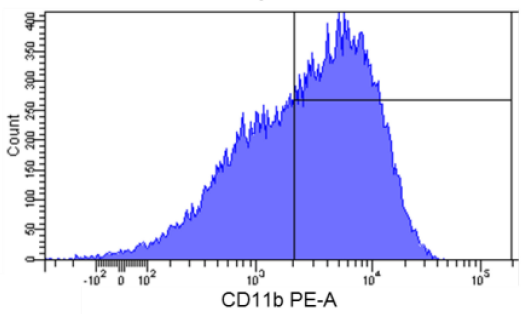

Figure 3 - Monocyte isolation is highly efficient and does not result in monocyte activation

(A) Monocytes were isolated from PBMC fractions. Quantification indicated that the hardly any monocytes were left in the CD14 negative (-) fraction, while the CD14 positive (+) fraction was very pure. This is also shown in the flow cytometry dot plots for the total PBMC and CD14+ fraction. (B) Histograms showing fluorescence intensity for CD11b of isolated monocytes at baseline (left panel) and after activation with $100 \mu \mathrm{M}$ LPS (right panel).

\section{CD14 isolation was effective and reproducible}

Isolation of monocytes resulted in highly pure $\mathrm{CD} 14^{+}$monocyte pools $(96.3 \% \pm$ $2.1 \%)$ at a recovery of more than $80 \%$ for all samples. Recovery was determined by calculating the residual $\mathrm{CD} 14^{+}$population in the $\mathrm{CD} 14$ negative 
fraction. Of note, there was hardly any spillover of CD14 negative cells in the isolated $\mathrm{CD} 14^{+}$monocyte pools (figure $3 \mathrm{~A}$ ).

The efficiency and purity observed for the isolation of fresh PBMCs did not differ from that obtained from frozen PBMCs. Freezing and thawing, either before or after CD14 isolation, did not show any effects on the CD14 $4^{+}$ monocyte surface expression of CD11b, CD62L, and CCR2 (data not shown), illustrating that membrane markers are well preserved during the experimental procedure. Activation potential of isolated monocytes was neither influenced by the isolation procedure nor by the freezing/thawing step, as all cells still could be efficiently activated and showed no baseline increase of CD11b expression (figure 3B).

To verify whether the clones selected on activated THP-1 monocytes also had a higher affinity for monocytes derived from UAP patients, whole cell ELISA was performed comparing phage binding to control and UAP monocytes, isolated by $\mathrm{CD}_{14}{ }^{+}$bead selection. Indeed, one clone (C10) showed significantly increased affinity for UAP-derived monocytes compared to monocytes from healthy individuals (figure 4A). 
A

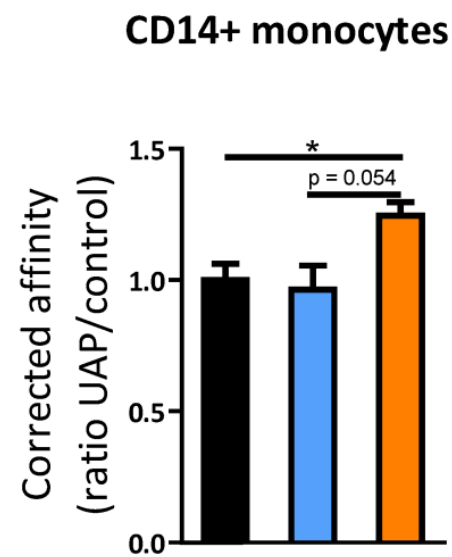

C
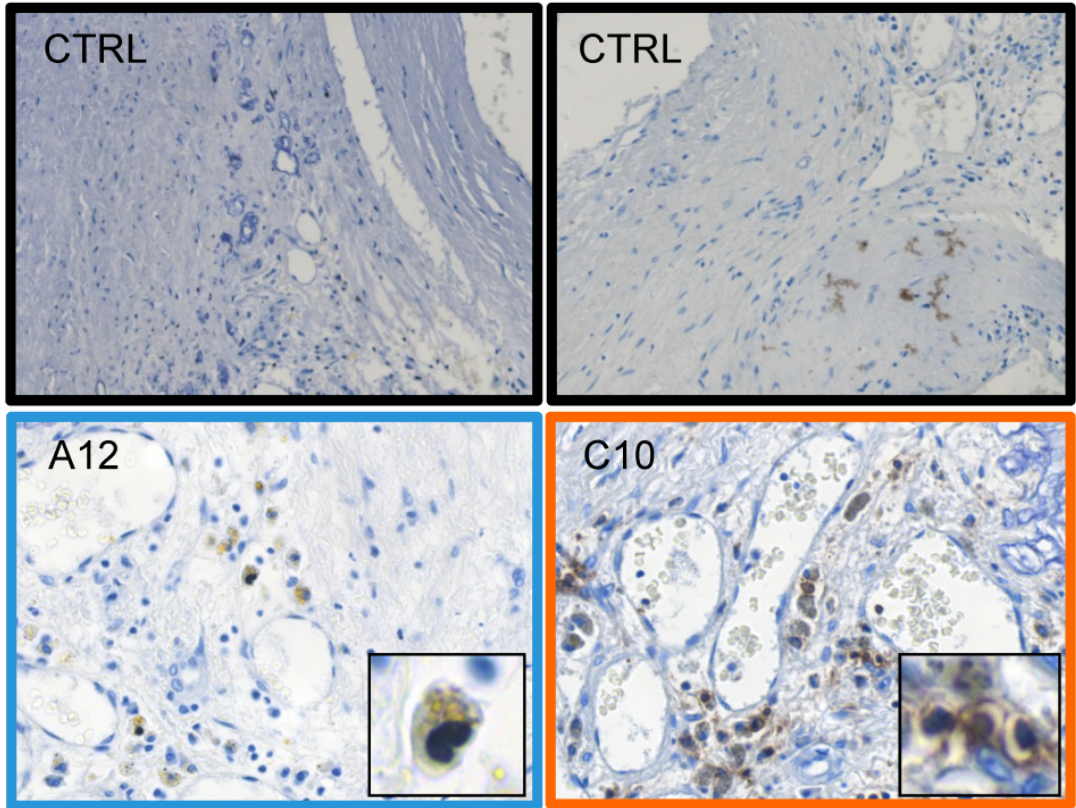

Figure 4 - Screenings on THP-1 cells, monocytes, and atherosclerotic lesions reveals that markers selected have biomarker potential

(A) Whole cell ELISA on human monocytes isolated from healthy controls and UAP patients showed increased affinity for UAP monocytes for some clones, compared to a control (G8) clone (B) Optical density (OD) of selected phages for binding on LPS-activated cells compared to control cells. Three clones were selected: G8C (Control; black bar), A12 (high affinity; blue bar), and C10 (intermediate affinity; orange bar). (C) Immunohistochemistry pictures for control (G8C), A12, and C10 clones on advanced atherosclerotic lesions. Inserts show positive cells for $\mathrm{A} 12$ and $\mathrm{C} 10$ staining. 
Despite, the enrichment observed for the total phage libraries per selection round (figure $2 \mathrm{~A}$ ), as well as the enrichment for the individual clones was rather limited (figure 2B), we investigated the capacity of selected clones to bind monocytic cells in atherosclerotic lesions. We selected two clones for further testing; clone A12 which showed high affinity for activated THP-1 cells and clone C10 displaying moderate affinity for activated cells but increased affinity for UAP monocytes, as well as clone G8 (control) which had no increased affinity for binding to activated THP-1 cells or UAP monocytes (figure $4 A+B$ ). Early lesions (intimal thickening), advanced lesions (thick fibrous cap atheroma), or advanced, vulnerable lesions (intraplaque hemorrhage/plaque rupture)were stained with selected clones, after which binding was detected using a HRP-conjugated anti-M13 antibody. Early lesions did not stain positive for any of the tested clones (data not shown). However, clone A12 as well as C10, but not G8 (control), gave detectable staining signal for advanced atherosclerotic lesions (figure $4 \mathrm{C}$ ).

Moreover, clones A12 and C10 were found to specifically stain cells in highly vascularized regions of the atherosclerotic plaque. Cells that showed positive staining were morphologically assigned to be monocytic cells, based on their size and nuclear shape. Interestingly, clones A12 and C10 showed a different staining pattern, with intra-cellular localized staining for A12, and clear plasma membrane staining for clone C10. Nevertheless, for both clones staining was very specific with regard to binding to monocytic cells in highly vascularized regions of advanced, vulnerable lesions.

\section{Discussion}

Diagnostic and prognostic markers used are generally based on levels of soluble proteins, lipids, carbohydrates, miRNAs, or levels of specific cell types in bodily fluids ${ }^{7,23}$. One of the most frequently used markers in the field of cardiovascular medicine is troponin ${ }^{24}$. This marker has very high specificity and also sensitivity for the diagnosis of an acute myocardial infarction (AMI) ${ }^{24,25}$. For atherosclerosis and more in particular unstable angina pectoris no (protein) markers have been defined until now and therefore diagnosis is merely based on clinical parameters and exclusion of $\mathrm{AMI}^{5}$. In this study we show that circulating monocytes carry information on local vascular disease status which could be applicable to diagnose or even predict (future) acute cardiovascular syndromes. 
Phage display is a powerful technique that yields high efficiency for the unbiased discovery of novel antibodies against known as well as unknown epitopes ${ }^{17}$. The technique has already been successfully applied in the cardiovascular field, to identify plaque proteins targeting autoantibodies ${ }^{26}$ and to design binders of known candidates for molecular imaging and intervention such as P-selectin ${ }^{27}$ and SR-Al ${ }^{28}$, or of whole cells ${ }^{29,30}$. In this study we applied phage display to identify antibodies able to differentiate between activated and non-activated on monocytes, for use in molecular diagnosis of unstable angina pectoris. Standard selection and screening protocols ${ }^{20}$ were adapted to be able to accommodate whole cells as template. One of the challenges to be met, especially in the validation phase, was that, contrary to most phage display efforts thus far, antibody and antigen both were unknown at the start of the study. We opted for activated THP-1 monocyte cells as selection platform, to circumvent interpatient variability; taken advantage of the recent findings that UAP and AMI patient monocytes show altered TLR4 response and/or basal activation status ${ }^{31-34}$, patient disease status was emulated by preactivating monocytes with the TLR4 agonist lipopolysaccharide (LPS).

For the verification stage we used patient-derived CD14+ monocytes. Importantly, the activation status of CD14+ monocytes was not impacted by cell isolation and CD14+ still retained full responsiveness to stimuli such as lipopolysaccharide (LPS). Thus, differential binding of phages to UAP monocytes versus monocytes from healthy volunteers was very likely reflecting disease state, although we cannot completely exclude that differences between patient groups in other parameters such as medication or diet could have had an effect on proteins expressed by circulating monocytes. Further validation in larger cohorts of risk factor matched patient groups will be required to address this point.

As TLR4 activation was applied for the initial biomarker discovery phase, the specificity of the binders found does not have to be restricted to monocytes in relation to advanced or unstable atherosclerosis. TLR4 has been implicated in rheumatoid arthritis ${ }^{35}$ and systemic lupus erythromatosus, however TLR4 appears to be non-involved or downregulated in these autoimmune diseases ${ }^{36,37}$. Still, TLR4 activation is implicated to be involved in multiple sclerosis ${ }^{38}$ and possibly asthma ${ }^{39,40}$. Therefore, TLR4 activation dependent biomarkers could contribute at least to some of the 
aforementioned diseases. Nevertheless, TLR4 signaling is most often linked to CVD , underpinning the relevance of our approach in a cardiovascular setting.

Monocytes have a pivotal role in all phases of atherosclerosis development ${ }^{41}$ and interact with the vessel wall ${ }^{42}$. These cells are the first cells to enter the initial lesion upon injury ${ }^{43}$, and the main source of macrophages which were seen to exert important functions in plaque progression $^{44}$ and eventual plaque destabilization, resulting in clinical manifestations such as myocardial infarction and stroke ${ }^{45}$. This key role in disease ontogenesis, combined with their innate patrolling function renders them highly sensitive sentinels of local vascular disease. Despite the cognition of macrophages as biomarker source for CVD status ${ }^{46}$, circulating monocytes have hardly been considered as carrier of information on plaque or vessel status, apart from their rolling and adhesion behavior. However, interaction with the diseased endothelium, as well as with blood containing cells and cytokines, can impact monocyte phenotype as we show in the current study. This notion concurs well with previous studies, which investigated diseaseassociated changes in monocyte expression and their potential as biomarker in cardiovascular diseases, as reviewed by Gratchev et al ${ }^{47}$. Different types of monocyte markers are described including monocyte (subset) numbers, protein markers, and functional markers. Both total monocyte counts ${ }^{48}$ as well as subpopulations ${ }^{49,50}$ (e.g. $\mathrm{CD}^{4} 4^{++} / \mathrm{CD} 16^{-}$monocytes) can function as biomarker for CVD. Further, activation markers as CD18, CD11b, and CXCR1 give information on morbidity in heart failure ${ }^{51}$. Finally, increased monocyteendothelial cell interactions ${ }^{52}$ and increased activation potential upon IL-4 stimulus ${ }^{47}$ can also give information on local atherogenic disease. The mere fact that our phage marker is able to distinguish between vulnerable and stable plaque monocytes is highly supportive of cross-interaction between lesion and monocyte.

To conclude we offer proof of concept of phage display aided identification of monocyte markers with increased affinity for circulating and plaque monocytes in unstable or advanced atherosclerosis. While holding promise, the actual target of the phage exposed antibody remains to be determined and its specificity assessed, before this observation can be translated into a clinically viable diagnostic test. Defining the exact epitope will also give a better insight in the disease associated downstream signaling events and its consequences for monocyte phenotype in vascular pathologies 
such as investigated in this study, but potentially also in other inflammatory diseases, especially since TLR4 activation is present in acute inflammatory conditions. Taken together, we present novel markers for circulating and plaque monocytes in patients with unstable angina which could contribute to improvement of diagnosis of disease.

\section{Acknowledgements}

This research was performed within the framework of CTMM, the Center for Translational Molecular Medicine, project CIRCULATING CELLS (grant 01C-102), and was supported by the Netherlands Heart Foundation. EB is recipient of the Established Investigator fellowship (grant \# 2003T201) of the Netherlands Heart Foundation. 


\section{References}

1. Roger VL, Go AS, Lloyd-Jones DM, et al. Heart disease and stroke statistics-2012 update: a report from the American Heart Association. Circulation. 2012;125(1):e2-e220.

2. Osborne JA, Stone PH. Recent advances in the understanding and management of stable and unstable angina pectoris and asymptomatic myocardial ischemia. Curr Opin Cardiol. 1994;9(4):448-456.

3. Wright RS, Anderson JL, Adams CD, et al. 2011 ACCF/AHA Focused Update of the Guidelines for the Management of Patients With Unstable Angina/ Non-STElevation Myocardial Infarction (Updating the 2007 Guideline): a report of the American College of Cardiology Foundation/American Heart Association Task Force on Practice Guidelines. Circulation. 2011;123(18):2022-2060.

4. Baturin P, Alivov Y, Molloi S. Spectral CT imaging of vulnerable plaque with two independent biomarkers. Phys Med Biol. 2012;57(13):4117-4138.

5. Koenig W, Khuseyinova N. Biomarkers of atherosclerotic plaque instability and rupture. Arterioscler Thromb Vasc Biol. 2007;27(1):15-26.

6. Narula J, Nakano M, Virmani R, et al. Histopathologic characteristics of atherosclerotic coronary disease and implications of the findings for the invasive and noninvasive detection of vulnerable plaques. J Am Coll Cardiol. 2013;61(10):10411051.

7. George J, Schwartzenberg S, Medvedovsky D, et al. Regulatory T cells and IL10 levels are reduced in patients with vulnerable coronary plaques. Atherosclerosis. 2012;222(2):519-523.

8. Tenaglia AN, Buda AJ, Wilkins RG, et al. Levels of expression of P-selectin, Eselectin, and intercellular adhesion molecule-1 in coronary atherectomy specimens from patients with stable and unstable angina pectoris. Am J Cardiol. 1997;79(6):742747.

9. Williams HJ, Fisher EA, Greaves DR. Macrophage differentiation and function in atherosclerosis: opportunities for therapeutic intervention? J Innate Immun. 2012;4(5-6):498-508.

10. Friesenhagen J, Viemann D, Borgeling $Y$, et al. Highly Pathogenic Influenza Viruses Inhibit Inflammatory Response in Monocytes via Activation of Rar-Related Orphan Receptor RORalpha. J Innate Immun. 2013.

11. Chen Z, Kim SJ, Chamberlain ND, et al. The Novel Role of IL-7 Ligation to IL-7 Receptor in Myeloid Cells of Rheumatoid Arthritis and Collagen-Induced Arthritis. J Immunol. 2013;190(10):5256-5266.

12. Wang SW, Lin TM, Wang CH, Liu HH, Houng JY. Increased toll-like receptor 2 expression in peptidoglycan-treated blood monocytes is associated with insulin resistance in patients with nondiabetic rheumatoid arthritis. Mediators Inflamm. 2012;2012:690525.

13. Koltsova EK, Ley K. How dendritic cells shape atherosclerosis. Trends Immunol. 2011;32(11):540-547.

14. Legein B, Temmerman L, Biessen EA, Lutgens E. Inflammation and immune system interactions in atherosclerosis. Cell Mol Life Sci. 2013. 
15. Shantsila E, Tapp LD, Wrigley BJ, Montoro-Garcia S, Lip GY. Receptors to interleukin- 6 and adhesion molecules on circulating monocyte subsets in acute myocardial infarction. Thromb Haemost. 2013;110(2).

16. Wrigley BJ, Shantsila E, Tapp LD, Lip GY. CD14++CD16+ monocytes in patients with acute ischaemic heart failure. Eur J Clin Invest. 2013;43(2):121-130.

17. Hoogenboom HR. Selecting and screening recombinant antibody libraries. Nat Biotechnol. 2005;23(9):1105-1116.

18. Cooksley-Decasper S, Reiser H, Thommen DS, et al. Antibody phage display assisted identification of junction plakoglobin as a potential biomarker for atherosclerosis. PLoS One. 2012;7(10):e47985.

19. Lee S, Yoon IH, Yoon A, Cook-Mills JM, Park CG, Chung J. An antibody to the sixth Ig-like domain of VCAM-1 inhibits leukocyte transendothelial migration without affecting adhesion. J Immunol. 2012;189(9):4592-4601.

20. Cao J, Zhao P, Miao XH, Zhao LJ, Xue LJ, Qi Zt Z. Phage display selection on whole cells yields a small peptide specific for HCV receptor human CD81. Cell Res. 2003;13(6):473-479.

21. Roovers RC, Laeremans T, Huang L, et al. Efficient inhibition of EGFR signaling and of tumour growth by antagonistic anti-EFGR Nanobodies. Cancer Immunol Immunother. 2007;56(3):303-317.

22. De Haard HJ, Bezemer S, Ledeboer AM, et al. Llama antibodies against a lactococcal protein located at the tip of the phage tail prevent phage infection. $J$ Bacteriol. 2005;187(13):4531-4541.

23. Tajika K, Okamatsu K, Takano M, et al. Malondialdehyde-modified low-density lipoprotein is a useful marker to identify patients with vulnerable plaque. Circ $J$. 2012;76(9):2211-2217.

24. Januzzi JL, Jr., Filippatos G, Nieminen M, Gheorghiade M. Troponin elevation in patients with heart failure: on behalf of the third Universal Definition of Myocardial Infarction Global Task Force: Heart Failure Section. Eur Heart J. 2012;33(18):22652271.

25. Katus HA, Remppis A, Neumann FJ, et al. Diagnostic efficiency of troponin T measurements in acute myocardial infarction. Circulation. 1991;83(3):902-912.

26. Cleutjens KB, Faber BC, Rousch M, et al. Noninvasive diagnosis of ruptured peripheral atherosclerotic lesions and myocardial infarction by antibody profiling. $J$ Clin Invest. 2008;118(8):2979-2985.

27. Molenaar TJ, Appeldoorn CC, de Haas SA, et al. Specific inhibition of Pselectin-mediated cell adhesion by phage display-derived peptide antagonists. Blood. 2002;100(10):3570-3577.

28. Segers FM, Yu H, Molenaar TJ, et al. Design and validation of a specific scavenger receptor class Al binding peptide for targeting the inflammatory atherosclerotic plaque. Arterioscler Thromb Vasc Biol. 2012;32(4):971-978.

29. Michon IN, Hauer AD, von der Thusen JH, et al. Targeting of peptides to restenotic vascular smooth muscle cells using phage display in vitro and in vivo. Biochim Biophys Acta. 2002;1591(1-3):87-97.

30. Brown KC. New approaches for cell-specific targeting: identification of cellselective peptides from combinatorial libraries. Curr Opin Chem Biol. 2000;4(1):16-21. 
31. Versteeg D, Hoefer IE, Schoneveld AH, et al. Monocyte toll-like receptor 2 and 4 responses and expression following percutaneous coronary intervention: association with lesion stenosis and fractional flow reserve. Heart. 2008;94(6):770-776.

32. de Kleijn D, Pasterkamp G. Toll-like receptors in cardiovascular diseases. Cardiovasc Res. 2003;60(1):58-67.

33. Kashiwagi $M$, Imanishi $T$, Ozaki $Y$, et al. Differential expression of Toll-like receptor 4 and human monocyte subsets in acute myocardial infarction. Atherosclerosis. 2012;221(1):249-253.

34. Tapp LD, Shantsila E, Wrigley BJ, Montoro-Garcia S, Lip GY. TLR4 expression on monocyte subsets in myocardial infarction. J Intern Med. 2013;273(3):294-305.

35. Choe JY, Crain B, Wu SR, Corr M. Interleukin 1 receptor dependence of serum transferred arthritis can be circumvented by toll-like receptor 4 signaling. J Exp Med. 2003;197(4):537-542.

36. Sanchez E, Orozco G, Lopez-Nevot MA, Jimenez-Alonso J, Martin J. Polymorphisms of toll-like receptor 2 and 4 genes in rheumatoid arthritis and systemic lupus erythematosus. Tissue Antigens. 2004;63(1):54-57.

37. Kirchner M, Sonnenschein A, Schoofs S, Schmidtke P, Umlauf VN, MannhardtLaakmann $W$. Surface expression and genotypes of Toll-like receptors 2 and 4 in patients with juvenile idiopathic arthritis and systemic lupus erythematosus. Pediatr Rheumatol Online J. 2013;11(1):9.

38. Waldner $\mathrm{H}$, Collins $\mathrm{M}$, Kuchroo VK. Activation of antigen-presenting cells by microbial products breaks self tolerance and induces autoimmune disease. J Clin Invest. 2004;113(7):990-997.

39. Fageras Bottcher M, Hmani-Aifa M, Lindstrom A, et al. A TLR4 polymorphism is associated with asthma and reduced lipopolysaccharide-induced interleukin-12(p70) responses in Swedish children. J Allergy Clin Immunol. 2004;114(3):561-567.

40. Yang IA, Barton SJ, Rorke S, et al. Toll-like receptor 4 polymorphism and severity of atopy in asthmatics. Genes Immun. 2004;5(1):41-45.

41. Hilgendorf I, Swirski FK. Making a difference: monocyte heterogeneity in cardiovascular disease. Curr Atheroscler Rep. 2012;14(5):450-459.

42. Weyrich AS, Mclntyre TM, McEver RP, Prescott SM, Zimmerman GA. Monocyte tethering by P-selectin regulates monocyte chemotactic protein-1 and tumor necrosis factor-alpha secretion. Signal integration and NF-kappa B translocation. J Clin Invest. 1995;95(5):2297-2303.

43. Gerrity RG. The role of the monocyte in atherogenesis: I. Transition of bloodborne monocytes into foam cells in fatty lesions. Am J Pathol. 1981;103(2):181-190.

44. Swirski FK, Pittet MJ, Kircher MF, et al. Monocyte accumulation in mouse atherogenesis is progressive and proportional to extent of disease. Proc Natl Acad Sci U S A. 2006;103(27):10340-10345.

45. Halvorsen B, Otterdal K, Dahl TB, et al. Atherosclerotic plaque stability--what determines the fate of a plaque? Prog Cardiovasc Dis. 2008;51(3):183-194.

46. Gui T, Shimokado A, Sun Y, Akasaka T, Muragaki Y. Diverse roles of macrophages in atherosclerosis: from inflammatory biology to biomarker discovery. Mediators Inflamm. 2012;2012:693083.

47. Gratchev A, Sobenin I, Orekhov A, Kzhyshkowska J. Monocytes as a diagnostic marker of cardiovascular diseases. Immunobiology. 2012;217(5):476-482. 
48. Maekawa Y, Anzai T, Yoshikawa T, et al. Prognostic significance of peripheral monocytosis after reperfused acute myocardial infarction:a possible role for left ventricular remodeling. J Am Coll Cardiol. 2002;39(2):241-246.

49. Rothe G, Gabriel H, Kovacs E, et al. Peripheral blood mononuclear phagocyte subpopulations as cellular markers in hypercholesterolemia. Arterioscler Thromb Vasc Biol. 1996;16(12):1437-1447.

50. Barisione C, Garibaldi S, Ghigliotti G, et al. CD14CD16 monocyte subset levels in heart failure patients. Dis Markers. 2010;28(2):115-124.

51. Dixon DL, Griggs KM, Bersten AD, De Pasquale CG. Systemic inflammation and cell activation reflects morbidity in chronic heart failure. Cytokine. 2011;56(3):593-599. 52. Alderson LM, Endemann G, Lindsey S, Pronczuk A, Hoover RL, Hayes KC. LDL enhances monocyte adhesion to endothelial cells in vitro. Am J Pathol. $1986 ; 123(2): 334-342$. 

$: \because$

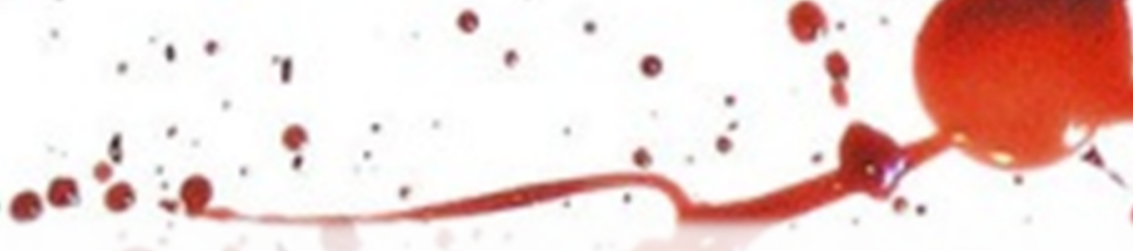

D.P.

Chapter 06

\section{Circulating unstable angina ${ }^{\circ} \cdot \circ$} pectoris patient monocytes 8 .

\section{harbor no basal transcriptomic}

\section{differences,}

Marco Manca, Jeroen JT Otten, Elisabeth McClellan, Nynke van den Akker, $>\bullet \bullet$ Mick Gagliardi, Fabienne Jeukens, Johannes Waltenberger, Johan Kuiper, Anton-Jan Zonneveld, Imo Hoefer, J Wouter Jukema, Gerard Pasterkamp,

and Erik AL Biessen

Submitted

c.

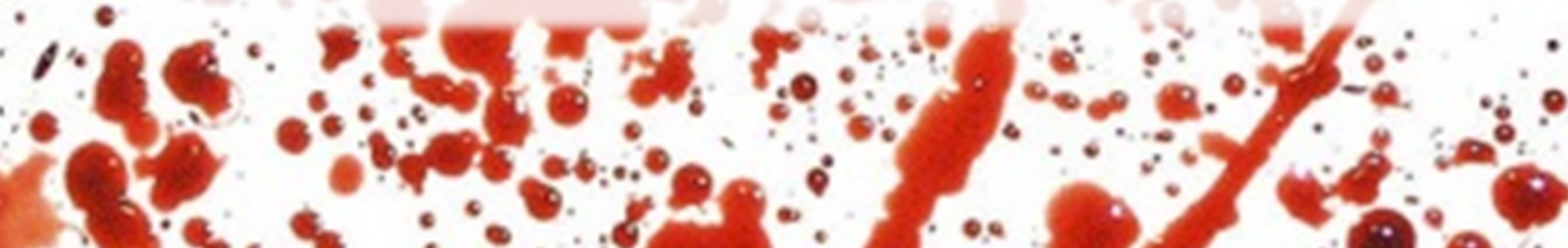

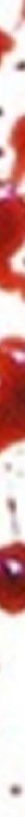




\begin{abstract}
Angina pectoris is considered to be one of the hallmarks of advanced cardiovascular disease and it often precedes ischemic events such as myocardial infarction and stroke. The current study investigates the differences between stable and unstable angina pectoris on a transcriptomics/genomic level to determine processes involved and to discover biomarkers to distinguish between stable and unstable angina pectoris. Circulating monocytes from patients included in the CTMM Circulating Cells cohort were isolated upon admission to the hospital. Monocytes were analyzed on a cellular level by flow cytometry and on a genomic level using micro-array analysis. Both the analysis on a proteomic and genomic level indicated the absence of distinct differences between the stable and unstable angina pectoris patients, however both analyses indicated the involvement of migratory pathways, as chemokine and cytokine signaling were limitedly affected. Despite major clinical differences between patients suffering from stable and unstable angina, we were unable to segregate these patient populations on a cellular or genetic level, indicative of only minor alterations of circulating monocytes in response to local atherosclerotic vessel disease.
\end{abstract}




\section{Introduction}

Cardiovascular diseases are one of the leading causes of death in Western societies and prevalence is increasing in developing countries ${ }^{1}$. Despite the growing public health efforts in preventive medicine, most patients will be referred to the hospital when already suffering clinical symptoms such as chest pain. These symptoms, also referred to as angina pectoris, are caused by reduced availability of oxygen (and nutrients) to the myocardium. Almost ubiquitously the underlying cause is represented by stenosis or impaired dynamics of the coronaries due to atherosclerotic disease ${ }^{2}$. Distinction between stable angina pectoris (SAP) and unstable angina pectoris (UAP) is made based on clinical history and semeiotics. Since this is a subjective process, and plaque stability in general is an important variable when it comes to the prognosis of a patient with angina, there is increasing need for alternative markers which can be used to classify angina pectoris into stable or unstable.

Preferably, such markers should also be able to quantitatively estimate the risk of developing future cardiovascular events, with high reproducibility, or at least reflect key processes in disease development. Additionally, it would desirable to identify circulating biomarkers, easily measurable with an easy and stress-free blood sampling procedure, to minimize the physical burden for patients and to seamlessly combine testing within the already established clinical procedures (e.g. troponin measurement ${ }^{3}$ ). Further, markers for SAP and UAP should have prognostic features to ensure optimal assessment of the long term risk of cardiovascular events and develop personalized medicine strategies.

In recent years, biomarker research has broadened its focus to also include cellular markers besides more classical (soluble) circulating proteins. Established protein biomarkers include C-reactive protein (CRP) for acute coronary syndrome ${ }^{4,5}$, but also cytokines like growth differentiation factor 15 (GDF15) for CVD in general ${ }^{6}$. Recently, increasing numbers of various UAP specific biomarkers have been proposed, including serum levels of placental growth factor ${ }^{7}$, deoxyribonuclease $1^{8}$, matrix metalloproteinase (MMP) $8^{\text {ref } 9}$. Cytokines, like soluble CD40 ${ }^{\text {ref }}{ }^{10}$, pro-brain type natriuretic peptide (BNP) ${ }^{11}$, and myeloperoxidase (MPO) ${ }^{12}$, have also been suggested as biomarkers for CVD. Furthermore, monocyte subset $\left(\mathrm{CD} 14^{+} / \mathrm{CD} 16^{+} / \mathrm{CX}_{3} \mathrm{CR}^{+}\right)$counts ${ }^{13}$, as well as levels of specific membrane markers as the fractalkine receptor $\left(C_{3} C R 1\right)^{14}$ 
have been linked to plaque stability in UAP patients. Nevertheless, most of these markers are either restricted to specific patients populations ${ }^{15}$, or influenced by drug use ${ }^{13}$, limiting the clinical application as biomarker.

Novel cellular markers for CVD are circulating endothelial cells, which could be promising prognostic biomarkers ${ }^{16}$. Also markers on circulating leukocytes have been shown to have potential as diagnostic or even prognostic CVD biomarker. In fact, CD14 expression on monocytes is commonly studied to determine monocyte activation and disease progression ${ }^{17-19}$, however the results are still contradictive. It has been shown that inflammatory membrane markers such as toll-like receptors ${ }^{20,21}$ and CD11b/CD18 ${ }^{\text {ref } 22,23}$ are also associated with progression of CVD in general. However, many biomarker studies have a lack of discriminative power, are not specific to cardiovascular disease, or do not show diagnostic potential and risk prediction for individual patients.

These data nevertheless demand a more elaborate screening of proteome and transcriptome on relevant leukocyte species. As cells from the monocyte/macrophage lineage are accepted as one of the key players in atherosclerosis development, we focused on the potential of circulating monocytes as biomarker for angina pectoris. Furthermore, a transcriptome approach is very suitable for identifying novel biomarkers ${ }^{24}$, and can also give insight in underlying pathway regulation ${ }^{25,26}$. Here, we present data on transcriptomics analysis on UAP patient monocytes compared to SAP monocytes to identify novel targets for diagnosis and prognosis.

\section{Materials \& Methods}

\section{Patients}

This study included 25 patients with stable angina pectoris and 25 patients with unstable angina pectoris from the Center for Translational Molecular Medicine (CTMM) - Circulating Cells Cohort that were presented to the Maastricht University Medical Center, The Netherlands between July 2009 and June 2011. Patient groups were matched for age, sex, smoking habit, dyslipidemia, and hypertension (table 1). As negative control, 11 healthy individuals without any known cardiovascular disease were included. These controls presented to the hospital with chest pain of suspected cardiovascular origin, but did not show any overt stenosis and scored negative on ST elevation and/or troponin $\mathrm{T}(\mathrm{TnT})$. All participants provided written informed consent 
prior to participation. This study was approved by the local ethics committee. Exclusion criteria were active inflammatory conditions, autoimmune disease, malignancies, use of immunosuppressive drugs, and known hematological disorders. Patients with ST-elevation myocardial infarction (STEMI) were also excluded. Blood of patients with suspected unstable angina or non-STEMI was studied but retrospectively excluded from this analysis.

\section{Cell Isolation}

Upon inclusion, blood samples were collected in ethylenediaminetetraacetic acid (EDTA) anti-coagulated vacuum tubes and processed according to standardized procedures to analyze leukocyte subsets. In short, blood was transferred to a $50 \mathrm{~mL}$ tube (Greiner) and centrifuged for 15 minutes at $156 \mathrm{x} \mathrm{g}$ without brake. Subsequently, plasma was removed and $30 \mathrm{~mL}$ PBS-diluted blood was added on top of $15 \mathrm{~mL}$ Ficoll-Paque plus (Sigma) and spun for 20 minutes at $1000 \mathrm{x} g$ without brake. The leukocyte-enriched interphase was collected and washed twice with $\mathrm{PBS}$ before $\mathrm{CD} 14^{+}$monocyte isolation according to the manufacturers protocol (BD Bioscience). Isolated CD14 ${ }^{+}$ monocytes were gently frozen and stored at $-80^{\circ} \mathrm{C}$ until further use.

\section{Flow Cytometry}

To assess expression of surface markers $50 \mu \mathrm{l}$ of heparin anti-coagulated whole blood was incubated with fluorescent antibodies (supplemental table 1) for 30 minutes. After washing and whole blood erythrocyte lysis, the samples were analyzed by flow cytometry (Beckman Coulter FC 500). Granulocytes were gated based on their scatter properties, and confirmed based on CD16 expression. Monocytes were identified based on their scatter properties and monocyte subsets were quantified (percentage positive cells and mean fluorescence intensity $[\mathrm{MFI}]$ )based on surface expression of CD14, CD16, CCR2, and $\mathrm{CX}_{3} \mathrm{CR} 1$.

\section{RNA Isolation and Micro-Array Analysis}

Monocyte samples were shipped to AROS (Aarhus, Denmark) for RNA isolation and subsequent micro-array analysis. RNA was isolated using Illumina TotalPrep RNA Amplification Kit (Illumina, San Diego, CA, USA) and cDNA was produced. The RNA quality and integrity was very good, as the lowest RNA integrity number (RIN) was 8.3 besides on outlier with a RIN of 2.4. The 
average RIN was $9.22 \pm 0.14$, indicative of very good quality of the RNA samples.

Next, labeled cRNA was prepared and used on the array for hybridization according to manufacturer's instructions. HumanHT-12 v4 Bead chips were scanned by Illumina BeadStation (Illumina Inc., San Diego, CA, USA). Raw image analysis and signal extraction was performed with Illumina Beadstudio Gene Expression software with default settings (no background subtraction) and no normalization. Data were exported as text files.

\section{Computational methods}

Raw data were imported in $\mathrm{R}^{\text {ref } 27}$ and analyzed within the Bioconductor suite 28. Quality control, background correction, and robust spline normalization have been performed by lumi ${ }^{29}$. Differential expression analysis was performed by fitting a linear model to the paired set of expression values, and by applying a Bayesian empirical method for shrinkage of the standard errors towards a common value, in limma ${ }^{30}$. P-values have been adjusted for False Detection Rate $^{31}$.

To evaluate the reproducibility of our differential expression analysis, the package GeneSelector ${ }^{32}$ was used. First the differential expression test was repeated using a set of diverse statistical methods (Wilcoxon statistics empirical Bayes ${ }^{33}$, Wilcoxon statistics, Bayesian t-statistics ${ }^{34}$, and SAM ${ }^{35}$ ). Finally we repeated the analysis for altered (jackknife, label exchange, bootstrapping, and noise addition) datasets, to estimate the bias and variance of the data. A principal component analysis was ran to explore the relationship among the samples. Arbitrarily selecting the top 500 genes from the differential expression analysis results, pathway enrichment analysis was performed using signaling pathway impact analysis (SPIA) ${ }^{36}$ which leverages on a system biology approach to integrate information concerning gene ID, differential expression and pathway topology to calculate the pathway significance and its activation or inhibition. 


\section{Statistical Analysis}

All data on patient characteristics and flow cytometry are presented as mean \pm SEM. To compare individual groups, 2-tailed Students t-test was used; nonparametric data were analyzed using Mann-Whitney $U$ test; discrete data was analyzed using Chi square test. All analyses on patient characteristics and flow cytometry were performed using GraphPad Prism 5 software (GraphPad Software Inc, La Jolla, CA, USA) or IBM SPSS statistics 20 (IBM, Amsterdam, The Netherlands). p-values $<0.05$ were considered statistically significant.

\section{Results}

\section{Patient characteristics}

In total 25 stable angina pectoris (SAP) patients, 25 unstable angina pectoris (UAP) patients, and 11 controls were included in this study. However one UAP patient was reclassified as SAP by the clinicians after initial selection procedure was performed. A second UAP patient was excluded as no adequate monocyte sample was available. As a result the study comprised 23 UAP patients, 26 risk factor-matched SAP patients, and 11 controls for final analysis.

\section{Table 1 - Patient characteristics}

Baseline characteristics for healthy controls (controls), stable angina pectoris patients (SAP), and unstable angina pectoris patients (UAP). Indicated is: number (percentage of group), except for Age (mean); BMI = Body Mass Index, MI = Myocardial Infarction

\begin{tabular}{lllll}
\hline & Controls & SAP & UAP & $p$-value \\
\hline & $\mathbf{N}=\mathbf{1 1}$ & $\mathbf{N}=\mathbf{2 6}$ & $\mathbf{N}=\mathbf{2 3}$ & \\
Age & 59.55 & 63.23 & 63.7 & 0.50 \\
Sex & $7(63.6)$ & $14(53.8)$ & $13(56.5)$ & 0.86 \\
BMI & 28.34 & 26.92 & 26.97 & 0.70 \\
Smoker & $3(27.3)$ & $9(34.6)$ & $11(47.8)$ & 0.45 \\
Hypertension & $4(36.4)$ & $15(57.7)$ & $16(69.6)$ & 0.18 \\
Diabetes Mellitus & $2(18.2)$ & $3(11.5)$ & $7(30.4)$ & 0.25 \\
Dyslipidemia & $5(45.5)$ & $21(80.8)$ & $16(69.6)$ & 0.10 \\
Peripheral artery disease & $2(18.2)$ & $3(11.5)$ & $3(13.0)$ & 0.86 \\
Previous MI & $0(0.0)$ & $7(26.9)$ & $3(13.0)$ & 0.11 \\
Cerebrovascular attack & $0(0.0)$ & $2(7.7)$ & $5(21.7)$ & 0.13 \\
\hline
\end{tabular}


No statistically significant differences in patient characteristics were detected for age, sex, and body mass index (BMI) between healthy controls, SAP and UAP patients (table 1). In the healthy control group 7 patients were male (63.6\%) and the mean age was 59.6 years ( $59.6 \pm 3.2$ years). It should be noted that accepted risk factors as smoking, hypertension, diabetes mellitus, and dyslipidemia, additional matching criteria for patient group buildup, did not differ between groups (table 1). Despite the absence of previous myocardial infarction and cerebrovascular attacks in the control group, no statistically significant difference could be observed between healthy controls, SAP, and UAP patients (table 1).

\section{Leukocyte counts are unaltered between SAP and UAP patients}

No statistically significant differences were observed in total leukocyte counts between controls, SAP, and UAP patients (table 2). Also lymphocyte and granulocyte counts were unchanged between controls and patient groups (table 2). Both lymphocytes and granulocytes tended to increase with severity of disease (controls $\rightarrow$ SAP $\rightarrow U A P$ ), albeit these results did not reach significance. Of note, monocyte counts were significantly reduced in SAP patients compared to both healthy controls and UAP patients, but did not differ between the latter two groups (table 2).

\section{Table 2 - Leukocyte profile of patients included}

Absolute cell counts from healthy controls (controls), stable angina pectoris patients (SAP), and unstable angina pectoris patients (UAP) upon inclusion. Indicated is mean \pm SEM. $* p<0.05$ vs UAP

\begin{tabular}{lllll}
\hline & Controls & SAP & UAP & $p$-value \\
\hline & $\mathbf{N}=\mathbf{1 1}$ & $\mathbf{N}=\mathbf{2 6}$ & $\mathbf{N}=\mathbf{2 3}$ & \\
Leukocytes & $7.00( \pm 0.42)$ & $6.90( \pm 0.66)$ & $7.47( \pm 0.80)$ & 0.82 \\
Lymphocytes & $1.66( \pm 0.17)$ & $1.83( \pm 0.19)$ & $2.27( \pm 0.20)$ & 0.15 \\
Monocytes & $0.55( \pm 0.04)$ & $0.39( \pm 0.03)^{*}$ & $0.51( \pm 0.04)$ & $<\mathbf{0 . 0 1}$ \\
Neutrophils & $4.68( \pm 0.65)$ & $5.35( \pm 0.35)$ & $5.99( \pm 0.42)$ & 0.23 \\
\hline
\end{tabular}




\section{Flow cytometry excludes polarization bias between patient groups}

Flow cytometry analysis confirmed the above findings, in that there were no differences in granulocytes between stable and unstable angina (figure $1 \mathrm{~A}$ ). Relative total monocyte counts measured by flow cytometry (figure 1B), as well as $\mathrm{CD} 14^{\text {high }} \mathrm{CD} 16^{-}$and $\mathrm{CD} 14^{+} \mathrm{CD} 16^{+}$monocyte subsets were unaltered in UAP versus SAP patients (figure $1 C+D$ ). The $C C R 2$ and $C_{3} C R 1$ chemokine receptor density on $\mathrm{CD} 14^{\text {high }} \mathrm{CD} 16^{-}$and $\mathrm{CD} 14^{+} \mathrm{CD} 16^{+}$was not affected as mean fluorescence intensity (MFI) for these chemokine receptors on both monocyte subsets was unaltered in SAP and UAP patients (table 3).

A

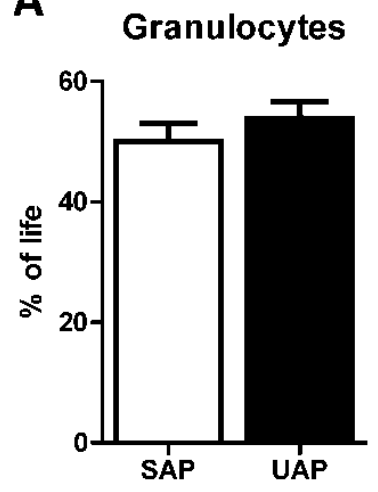

C
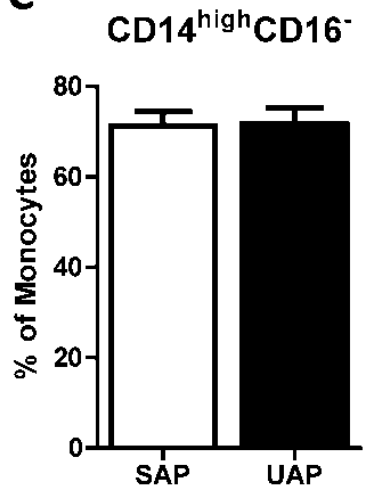

B

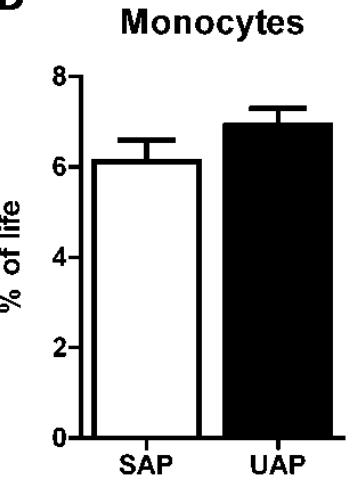

D

$\mathrm{CD}^{+}{ }^{+} \mathrm{CD}^{+}{ }^{+}$

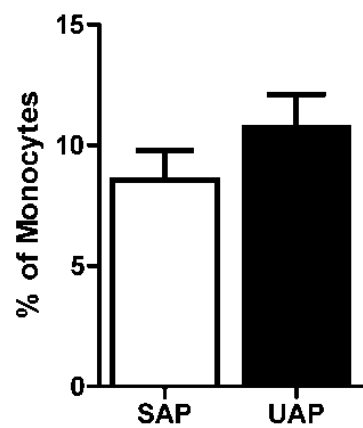

Figure 1 - Flow cytometry indicates no differences in chemokine receptor expression.

Flow cytometry data showing (A) granulocytes, (B) total monocytes, (C) CD $14^{\text {high }} \mathrm{CD} 16^{-}$ monocytes, and (D) $\mathrm{CD} 14^{+} \mathrm{CD} 16^{+}$monocytes for SAP and UAP patients. White bars are stable angina pectoris patients (SAP) and black bars are unstable angina pectoris (UAP) patients. ${ }^{*} p<$ 0.05 and ${ }^{* *} p<0.01$ 
Table 3 - Corrected mean fluorescence intensity for chemokine receptor expression on monocyte subsets

\begin{tabular}{llllll}
\hline Corrected MFI & & Controls & SAP & UAP & $p$-value \\
\hline & & $\mathbf{N}=\mathbf{1 1}$ & $\mathbf{N}=\mathbf{2 6}$ & $\mathbf{N}=\mathbf{2 3}$ & \\
$\mathrm{CD} 14^{\text {high }} \mathrm{CD}^{-}$ & $\mathrm{CCR} 2$ & $0.283( \pm 0.011)$ & $0.288( \pm 0.007)$ & $0.284( \pm 0.009)$ & 0.93 \\
& $\mathrm{CX}{ }_{3} \mathrm{CR} 1$ & $0.290( \pm 0.003)$ & $0.293( \pm 0.004)$ & $0.289( \pm 0.003)$ & 0.52 \\
$\mathrm{CD} 14^{+} \mathrm{CD} 16^{+}$ & $\mathrm{CCR2}$ & $0.309( \pm 0.022)$ & $0.297( \pm 0.013)$ & $0.304( \pm 0.010)$ & 0.84 \\
& $\mathrm{CX}{ }_{3} \mathrm{CR} 1$ & $0.339( \pm 0.005)$ & $0.344( \pm 0.004)$ & $0.344( \pm 0.003)$ & 0.56 \\
\hline
\end{tabular}

\section{Principal component analysis does not reveal any segregation between} patient groups

Flow cytometry based quality control for the monocyte isolation confirmed that CD14 positive monocytes could be isolated at high purity (>99.5\%) and moderate to high recovery ( $>80 \%$; figure $2 \mathrm{~A}$ ). Bead-purified monocytes were still viable and still able to react to subsequent lipopolysaccharide activation, indicating that the selection method did not (completely) activate the monocytes (figure 2B).

Density histogram of raw log-intensities from patient samples before (upper panel) and after (lower panel) preprocessing revealed that the micro array analyses were of good quality. This was confirmed by the overlap of samples after normalization, which indicates that batch effects have been eradicated and that all the signals share the same scale (figure $3 \mathrm{~A}$ ).

A

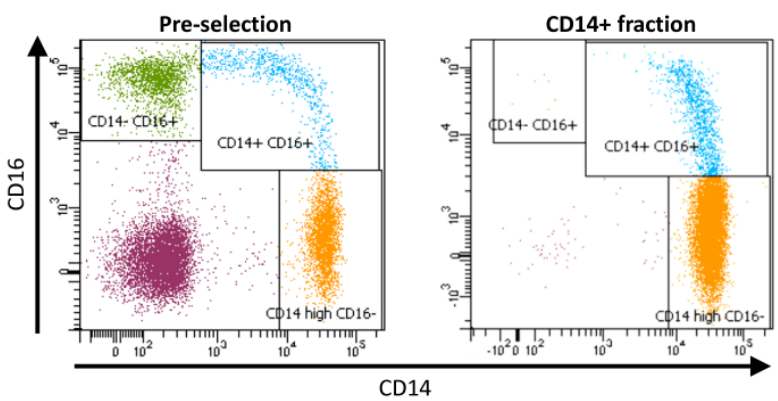

B

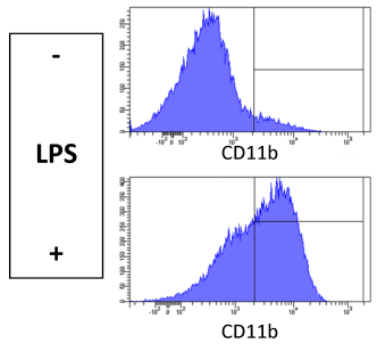

Figure $\mathbf{2}$ - Monocyte are isolated efficiently and without massive activation.

(A) Representative dot plots for monocyte population based on CD14 and CD16 expression before (left panel) and after (right panel) CD14+ bead isolation. (B) Histograms showing CD11b expression on isolated monocytes before (upper panel) and after (lower panel) stimulation with $100 \mathrm{ng} / \mathrm{mL}$ lipopolysaccharide (LPS). 
Initial principal component analysis (PCA) was performed on the raw data to identify whether the attributes of the data were able to show clustering of the datasets. PCA indicated that there was no segregation of the data based on the patients disease state (control, stable angina pectoris, unstable angina pectoris) before normalization and background correction (figure 3B). Normalization of the data and subsequent background correction, did not reveal additional information, as there was no clustering of patient groups (or controls) visible (figure $3 \mathrm{C}$ ).

\section{Monocyte show subtle differences on transcriptomics level}

Differential expression analysis, after false discovery rate (FDR) adjustment (to correct for multiple testing), was used to determine genes of interest for further analysis. Analysis revealed the presence of both up- and downregulated gene sets in UAP versus SAP patients after correction for the controls (figure 4A). However, after stringent multiple testing correction we could no longer observe any statistically significant gene sets (data not shown) for the used conditions ( $p<0.01$ and/or foldchange $>30 \%$ ).

To verify whether the results were influenced by the statistical test used for these analyses, multiple tests were compared, including Wilcoxon statistics empirical Bayes (WilcEbam), Wilcoxon statistics (Wilcoxon), Bayesian t-statistics (BaldiLongT), and statistical analysis of micro arrays (SAM). All tests, expect for WilcEbam, resulted in similar outcomes regarding macroscopic clustering of the results as our default procedure linear models for micro-array data (Limma), suggesting that our analysis was not biased by the procedure used for analysis (figure 4B). 
A

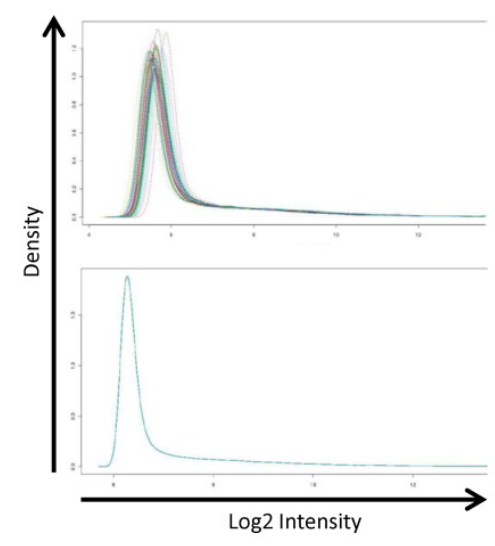

B

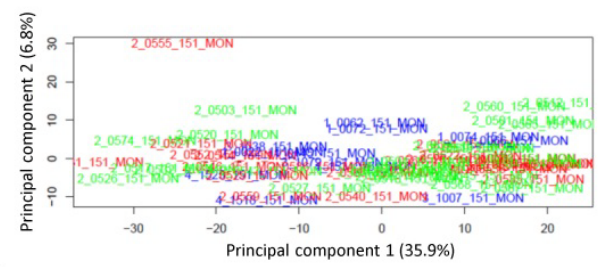

C

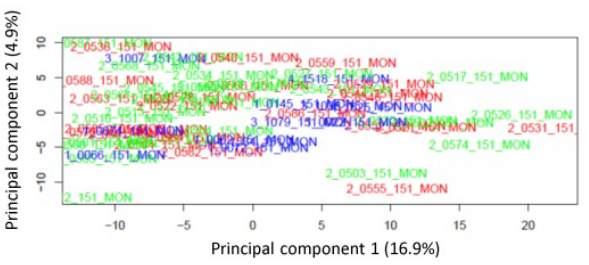

Figure 3 - Micro-array analysis does not result in clustering of patient groups.

(A) Micro-array analysis quality was assessed using density histograms of log2 intensity. Density histograms before (upper panel) and after (lower panel) processing are shown. Overlay of the lines after processing is indicative of good quality. $(\mathbf{B}+\mathbf{C})$ Principal component analysis (PCA) showed the absence of clustering of patient populations before $(\mathbf{B})$ and after $(\mathbf{C})$ background correction and normalization of the data.

A

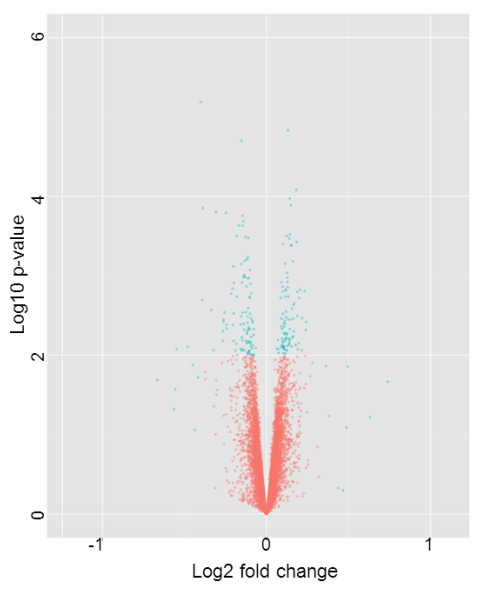

B

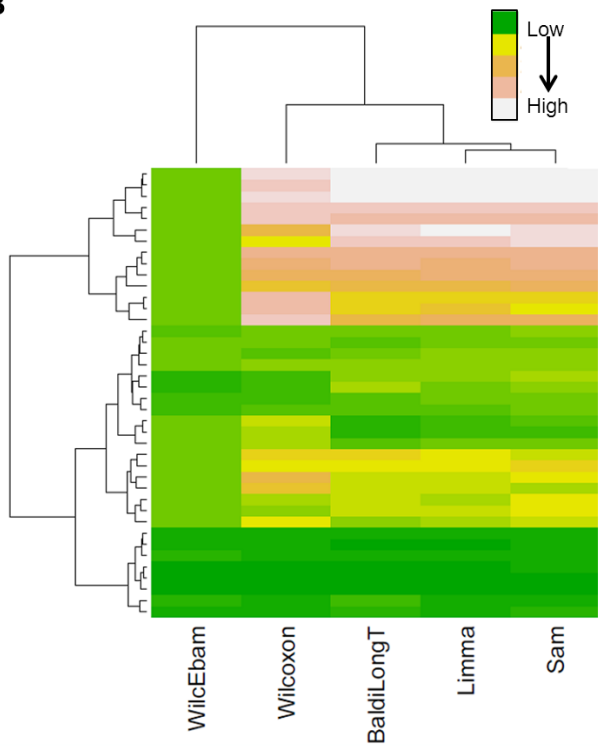

Figure 4 - Differential expression analysis indicate minute, yet significant differences.

(A) Volcano plot indicating differentially expressed genes (blue dots) with fold change over 30\% and/or p-value below 0.01. Red dots are genes not differentially expressed between SAP and UAP patients. (B) Macroscopical ordering of the results based on different statistical tests does not reveal test dependent bias of our results. 
Monocyte chemokine pathways are minimally affected in UAP patients

Pathway analysis, arbitrarily using the top 500 genes differentially expressed between SAP and UAP patient monocytes, indicated seven pathways which were significantly inhibited in UAP patients compared to SAP patients after correction for the controls. Two additional pathways were activated in UAP versus SAP patients (figure $5 \mathrm{~A}$ ). Four pathways remained significant after Bonferroni correction, and all nine were significant after FDR correction. The top four pathways were all immune-related: chemokine signaling pathway (inhibited; $\mathrm{p}<0.001$ ), cytokine-cytokine receptor interaction (inhibited; $\mathrm{p}<$ 0.01 ), vibrio cholera infection (inhibited; $p<0.05$ ), and epithelial cell signaling in $\mathrm{H}$. pylori infection (inhibited; $\mathrm{p}<0.05$; figure $5 \mathrm{~B}$ ). The other five pathways, Shigellosis (inhibited), cell cycle (inhibited), salivary secretion (inhibited), pathogenic E. coli infection (activated), and Alzheimer's disease (activated), did not pass Bonferroni correction (figure $5 A+B$ ). Besides cell division control protein 42 homolog (Cdc42) and adenylyl cyclase (AC), chemokines and chemokine receptor genes were most predominantly affected in the top dysregulated pathway (chemokine signaling pathway; figure 5C). 
A

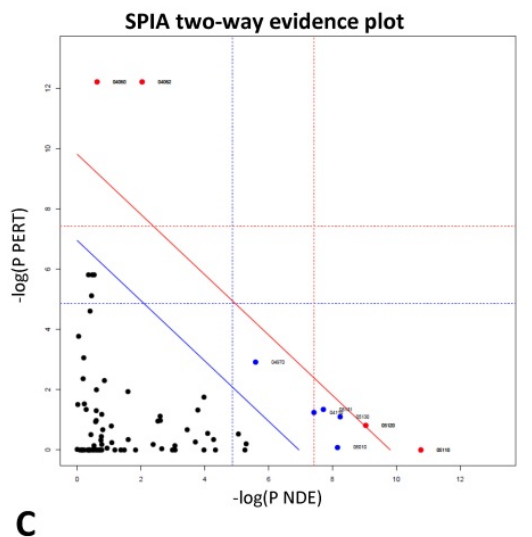

B

\begin{tabular}{lccc} 
Name Pathway & KEGG ID & Adjusted p-value & Status \\
\hline Chemokine Signaling Pathway & 04062 & 0.000834468 & Inhibited \\
Cytokine-Cytokine Receptor Interaction & 04060 & 0.003119522 & Inhibited \\
Vibrio Cholerae Infection & 05110 & 0.020780237 & Inhibited \\
Epithelial Cell Signaling in H. pylori Infection & 05120 & 0.048101923 & Inhibited \\
Pathogenic Escherichia coli Infection & 05130 & 0.076070121 & Activated \\
Shigellosis & 05131 & 0.098400310 & Inhibited \\
Cell Cycle & 04110 & 0.140056773 & Inhibited \\
Salivary Secretion & 04970 & 0.163562172 & Inhibited \\
Alzheimer's Disease & 05010 & 0.204885575 & Activated \\
\hline
\end{tabular}

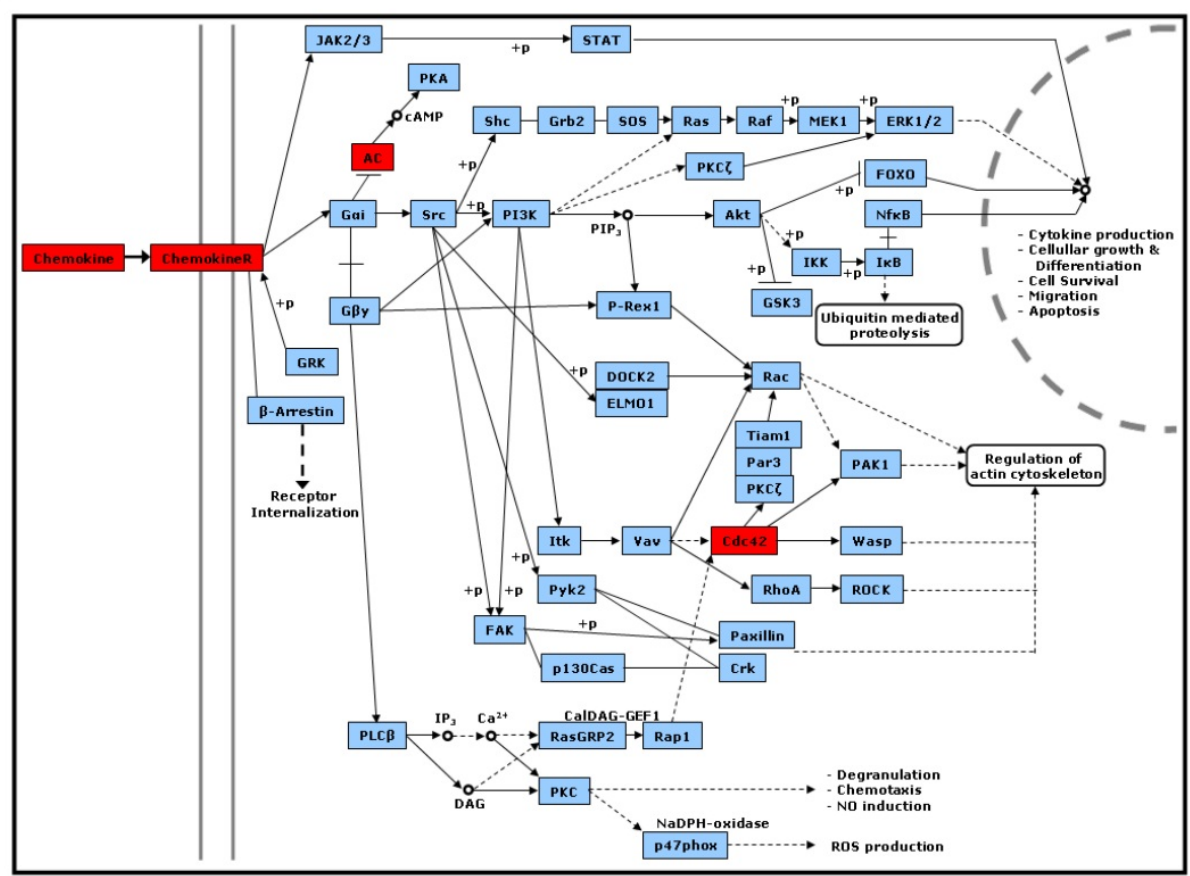

Figure 5 - Pathway analysis reveals subtle differences between SAP and UAP patients.

(A) SPIA pathway analysis reveals differentially regulated pathways between SAP and UAP patients based on Bonferroni correction (red dots) and FDR (blue dots). The red line and blue line indicate $p<0.05$ for Bonferroni and FDR correction, respectively. (B) Table indicating differentially regulated pathways including KEGG-ID, adjusted p-value, and status (activated/inhibited). (C) Chemokine pathway including the differentially expressed genes (red genes) within this pathway. 


\section{Discussion}

Distinguishing between stable angina pectoris (SAP) and unstable angina pectoris (UAP) patients at an early time point is essential for treatment of cardiovascular disease and risk prediction for future cardiovascular events. Furthermore, it is also essential to exclude patients with non-cardiovascular chest pain early to avoid additional, time-consuming, and expensive examinations and procedures. Various studies have investigated circulating or even cellular biomarkers to determine the disease state of the patient ${ }^{37-39}$. So far, no biomarker has been defined that is superior to commonly used clinical parameters, in discriminating SAP from UAP patients ${ }^{40,41}$. Cellular markers can not only give insight in the disease state of a patient, but can also contribute to understanding of underlying mechanisms.

The current study has explored the possibilities of using blood derived cellular markers for determination of disease state. The underlying notion is that circulating cells, and more in particular monocytes, can on the one hand sense the local and systemic milieu by either reacting to released cytokines ${ }^{42}$ and chemokines ${ }^{43}$, and on the other hand directly interact with the vessel wall 5,43-45, activating (patrolling) monocytes due to increased integrins in response to advanced atherosclerotic vessel disease ${ }^{44,46}$. Conceivably, the transient accumulation of activated platelets at lesioned foci in coronary arteries ${ }^{47}$, one of the processes underlying angina, will be sensed at an early stage by circulating monocytes. Moreover, dysregulated plasma cytokine and chemokine levels constitute a markedly altered microenvironment to which circulating monocytes are exposed. Overall, monocytes have the capacity to detect multiple targets associated with the pathogenesis of angina pectoris, and to respond to such stimuli on a cellular level.

The possibilities of using circulating leukocytes as target for biomarker discovery is not completely novel, as a limited number of studies has investigated peripheral blood mononuclear cells (PBMC) as messenger of atherosclerotic vessel disease using an transcriptomics approach ${ }^{48}$. Moore et al found a panel of differentially expressed genes, including genes involved in monocyte activation (CD14, TLR2) and scavenger receptors (CD163, CD36), which could identify stroke patients ${ }^{49}$. Furthermore, Patino et al identified Finkel-Biskis-Jinkins osteosarcoma (FOS) gene to be upregulated in circulating $\mathrm{CD} 14^{+}$monocytes from patients undergoing carotid endarterectomy compared 
to healthy controls ${ }^{50}$. However, in contrast to our study, these studies focused on endpoints (surgery and stroke) rather than diagnosis of patients at risk.

In this study, the SAP and UAP patients selected were matched for risk factors related to cardiovascular disease. The healthy controls did not suffer from cardiovascular disease before or upon inclusion. Leukocyte analysis indicated that total leukocytes, as well as lymphocytes and granulocytes were not different between healthy controls, SAP and UAP patients. Unexpectedly, circulating monocytes were slightly decreased in SAP patients, but not UAP patients, compared to healthy controls. Overall, patient demographics are not expected to have any influence on our analysis as parameters were very comparable between controls, SAP and UAP patients.

Flow cytometry did not show any differences in monocyte numbers, both total monocytes and subsets, as well as in chemokine receptor expression. With regard to monocytes, inflammatory $\mathrm{CD} 14^{\text {high }} \mathrm{CD} 16$ and patrolling $\mathrm{CD} 14^{+} \mathrm{CD} 16^{+}$monocyte subsets, we could not detect any differences in relative abundance. Recently $\mathrm{CD} 14^{+} \mathrm{CD} 16^{+}$monocyte enrichment was proposed as a measure of disease severity ${ }^{51}$, however this study did not differentiate between stable and unstable angina. On the protein level, we did not observe any changes in expression of two prototypic chemokine receptors instrumental in $\mathrm{CD} 14^{\text {high }} \mathrm{CD} 16^{-}$and $\mathrm{CD} 14^{+} \mathrm{CD} 16^{+}$trafficking, CCR2 and CX3CR1 as examined by flow cytometry. These data indicate that our transcriptomics analysis is not confounded by basal differences in SAP or UAP monocyte skewing. This supports, at least in part, the subtle differences in monocyte phenotype between SAP and UAP patients.

More detailed analysis of our transcriptomics data by principal component analysis failed to reveal clustering of patient groups. Previously, individual genes were suggested to be useful as a biomarker for coronary artery disease ${ }^{52,53}$, or as prognostic marker for disease progression ${ }^{54}$. However, as Venet et al showed individual genes tested might be as predictive for a specific disease as randomly chosen genes ${ }^{55}$. To prevent detection of non-specific genes, we conducted in-depth genome wide analysis to specifically detect gene clusters predictive for disease state. Differential expression analysis unveiled, in contrast to our flow cytometry data, subsets of genes to be up- or downregulated in unstable angina pectoris patients compared to stable angina pectoris patients after correction for the healthy controls, demonstrating that transcriptomic differences, though subtle, do 
exist between the patient populations. Pathway analysis indicated chemokine and cytokine pathways, as well as pathways related to infection and inflammation to be inhibited in UAP patients. This confirmed the notion that local ischemia and/or unstable atherosclerosis, and their resulting signals can impact circulating monocyte functions such as chemotaxis. The initially unexpected inhibition of chemokine - chemokine receptor interaction in UAP patients may also explain the elevated monocyte levels in this group compared to SAP patients, as the most response monocytes in UAP patients might be recruited to the site of injury, in this case the atherosclerotic lesion.

In conclusion, we were able to show that circulating cells do harbor hallmarks of local atherosclerotic disease, as UAP monocytes showed subtle transcriptomics differences on e.g. chemokine and cytokine receptor regulation compared to SAP monocytes. However, the detected effects are too small to justify circulating monocyte transcriptomics for biomarker derivation. Additional research is necessary to investigate the best approach to amplify or detect the subtle genomic or even downstream proteomic differences between SAP and UAP in a bed-side setting. 


\section{Supplemental data}

Supplemental table 1 - Antibodies for flow cytometry

\begin{tabular}{lll} 
Antibody & Company & Dilution \\
\hline CD14 PC7 & BD Biosciences & $1: 10$ \\
CD16 PC5 & BD Biosciences & $1: 10$ \\
CCR2 PE & BD Biosciences & $1: 5$ \\
CX ${ }_{3}$ CR1 FITC & BD Biosciences & $1: 5$ \\
CD3 ECD & BD Biosciences & $1: 10$ \\
CD4 PE-Cy7 & BD Biosciences & $1: 10$ \\
CD8 FITC & BD Biosciences & $1: 5$ \\
\hline
\end{tabular}




\section{References}

1. Mathers CD, Loncar D. Projections of global mortality and burden of disease from 2002 to 2030. PLoS Med. 2006;3(11):e442.

2. Libby P. Mechanisms of acute coronary syndromes and their implications for therapy. N Engl J Med. 2013;368(21):2004-2013.

3. Katus HA, Remppis A, Neumann FJ, et al. Diagnostic efficiency of troponin T measurements in acute myocardial infarction. Circulation. 1991;83(3):902-912.

4. Sheikh AS, Yahya S, Sheikh NS, Sheikh AA. C-reactive Protein as a Predictor of Adverse outcome in Patients with Acute Coronary Syndrome. Heart Views. 2012;13(1):7-12.

5. Lee $\mathrm{WH}$, Lee $\mathrm{Y}, \mathrm{Kim} \mathrm{JR}$, et al. Activation of monocytes, T-lymphocytes and plasma inflammatory markers in angina patients. Exp Mol Med. 1999;31(3):159-164.

6. Wang TJ, Wollert KC, Larson MG, et al. Prognostic utility of novel biomarkers of cardiovascular stress: the Framingham Heart Study. Circulation. 2012;126(13):15961604.

7. Lu HQ, Liang C, He ZQ, Fan M, Wu ZG. Circulating miR-214 is associated with the severity of coronary artery disease. J Geriatr Cardiol. 2013;10(1):34-38.

8. Fujibayashi K, Kawai $Y$, Kitayama M, et al. Serum deoxyribonuclease I activity can be a useful diagnostic marker for the early diagnosis of unstable angina pectoris or non-ST-segment elevation myocardial infarction. J Cardiol. 2012;59(3):258-265.

9. Momiyama $\mathrm{Y}$, Ohmori $\mathrm{R}$, Tanaka $\mathrm{N}$, et al. High plasma levels of matrix metalloproteinase- 8 in patients with unstable angina. Atherosclerosis. 2010;209(1):206-210.

10. Fouad HH, Al-Dera $\mathrm{H}$, Bakhoum SW, et al. Levels of SCD40 ligand in chronic and acute coronary syndromes and its relation to angiographic extent of coronary arterial narrowing. Angiology. 2010;61(6):567-573.

11. Ahluwalia N, Blacher J, Szabo de Edelenyi F, et al. Prognostic value of multiple emerging biomarkers in cardiovascular risk prediction in patients with stable cardiovascular disease. Atherosclerosis. 2013;228(2):478-484.

12. Tretjakovs $P$, Jurka $A$, Bormane $I$, et al. Circulating adhesion molecules, matrix metalloproteinase-9, plasminogen activator inhibitor-1, and myeloperoxidase in coronary artery disease patients with stable and unstable angina. Clin Chim Acta. 2012;413(1-2):25-29.

13. Imanishi T, Ikejima $\mathrm{H}$, Tsujioka $\mathrm{H}$, et al. Association of monocyte subset counts with coronary fibrous cap thickness in patients with unstable angina pectoris. Atherosclerosis. 2010;212(2):628-635.

14. Ikejima $H$, Imanishi $T$, Tsujioka $H$, et al. Upregulation of fractalkine and its receptor, CX3CR1, is associated with coronary plaque rupture in patients with unstable angina pectoris. Circ J. 2010;74(2):337-345.

15. Raaz-Schrauder D, Ekici AB, Munoz LE, et al. Patients with unstable angina pectoris show an increased frequency of the Fc gamma RIla R131 allele. Autoimmunity. 2012;45(7):556-564.

16. Damani S, Bacconi A, Libiger $O$, et al. Characterization of circulating endothelial cells in acute myocardial infarction. Sci Transl Med. 2012;4(126):126ra133. 
17. Lee WH, Lee Y, Jeong JO, Lee SY, Choi YH, Park JE. Activation of CD14 on circulating monocytes in patients with acute coronary syndrome. Int J Cardiol. 2001;80(2-3):135-142.

18. Zalai CV, Kolodziejczyk MD, Pilarski L, et al. Increased circulating monocyte activation in patients with unstable coronary syndromes. I Am Coll Cardiol. 2001;38(5):1340-1347.

19. Pasini AF, Anselmi M, Garbin U, et al. Enhanced levels of oxidized low-density lipoprotein prime monocytes to cytokine overproduction via upregulation of CD14 and toll-like receptor 4 in unstable angina. Arterioscler Thromb Vasc Biol. 2007;27(9):19911997.

20. Methe H, Kim JO, Kofler S, Weis $M$, Nabauer $M$, Koglin J. Expansion of circulating Toll-like receptor 4-positive monocytes in patients with acute coronary syndrome. Circulation. 2005;111(20):2654-2661.

21. Sels JW, Elsenberg EH, Hoefer IE, et al. Fractional flow reserve is not associated with inflammatory markers in patients with stable coronary artery disease. PLoS One. 2012;7(10):e46356.

22. De Servi S, Mazzone A, Ricevuti G, et al. Clinical and angiographic correlates of leukocyte activation in unstable angina. J Am Coll Cardiol. 1995;26(5):1146-1150.

23. Sanchez-Margalet V, Cubero JM, Martin-Romero C, Cubero J, Cruz-Fernandez JM, Goberna R. Expression of activation molecules in neutrophils, monocytes and lymphocytes from patients with unstable angina treated with stent implantation. Clin Chem Lab Med. 2004;42(3):273-278.

24. Zeller T, Wild P, Szymczak S, et al. Genetics and beyond--the transcriptome of human monocytes and disease susceptibility. PLoS One. 2010;5(5):e10693.

25. Rotival M, Zeller T, Wild PS, et al. Integrating genome-wide genetic variations and monocyte expression data reveals trans-regulated gene modules in humans. PLOS Genet. 2011;7(12):e1002367.

26. Schadt EE, Molony C, Chudin E, et al. Mapping the genetic architecture of gene expression in human liver. PLoS Biol. 2008;6(5):e107.

27. Team RDC. R: A language and environment for statistical computing. http://www.R-project.org. Veinna, Austria: R Foundation for Statistical Computing; 2008.

28. Gentleman RC, Carey VJ, Bates DM, et al. Bioconductor: open software development for computational biology and bioinformatics. Genome Biol. 2004;5(10):R80.

29. Du P, Kibbe WA, Lin SM. lumi: a pipeline for processing Illumina microarray. Bioinformatics. 2008;24(13):1547-1548.

30. Smyth G. Limma: linear models for microarray data. In: R. Gentleman VC, S. Dudoit, R. Irizarry, W. Huber ed. 'Bioinformatics and Computational Biology Solutions using R and Bioconductor'. New York: Springer; 2005:397-420.

31. Benjamini Y, Drai D, Elmer G, Kafkafi N, Golani I. Controlling the false discovery rate in behavior genetics research. Behav Brain Res. 2001;125(1-2):279-284.

32. Boulesteix AL SM. Stability and aggregation of ranked gene lists. Brief Bioinformatics. 2009;10(5):556-568.

33. Efron B, Tibshirani R. Empirical bayes methods and false discovery rates for microarrays. Genet Epidemiol. 2002;23(1):70-86. 
34. Baldi P, Long AD. A Bayesian framework for the analysis of microarray expression data: regularized $t$-test and statistical inferences of gene changes. Bioinformatics. 2001;17(6):509-519.

35. Tusher VG, Tibshirani R, Chu G. Significance analysis of microarrays applied to the ionizing radiation response. Proc Natl Acad Sci U S A. 2001;98(9):5116-5121.

36. Tarca AL, Draghici $S$, Khatri $P$, et al. A novel signaling pathway impact analysis. Bioinformatics. 2009;25(1):75-82.

37. Tsimikas S, Willeit P, Willeit J, et al. Oxidation-specific biomarkers, prospective 15-year cardiovascular and stroke outcomes, and net reclassification of cardiovascular events. J Am Coll Cardiol. 2012;60(21):2218-2229.

38. Kiechl S, Willeit J, Mayr M, et al. Oxidized phospholipids, lipoprotein(a), lipoprotein-associated phospholipase A2 activity, and 10-year cardiovascular outcomes: prospective results from the Bruneck study. Arterioscler Thromb Vasc Biol. 2007;27(8):1788-1795.

39. Mavromatis K, Aznaouridis K, Al Mheid I, et al. Circulating proangiogenic cell activity is associated with cardiovascular disease risk. J Biomol Screen. 2012;17(9):1163-1170.

40. Campeau L. Letter: Grading of angina pectoris. Circulation. 1976;54(3):522523.

41. Peterson E. The burden of angina pectoris and its complications [corrected]. Clin Cardiol. 2007;30(2 Suppl 1):I10-15.

42. Hojo Y, Ikeda U, Takahashi M, Shimada K. Increased levels of monocyterelated cytokines in patients with unstable angina. Atherosclerosis. 2002;161(2):403408.

43. Shi C, Pamer EG. Monocyte recruitment during infection and inflammation. Nat Rev Immunol. 2011;11(11):762-774.

44. Stolla M, Pelisek J, von Bruhl ML, et al. Fractalkine is expressed in early and advanced atherosclerotic lesions and supports monocyte recruitment via CX3CR1. PLoS One. 2012;7(8):e43572.

45. Mazzone A, De Servi S, Ricevuti G, et al. Increased expression of neutrophil and monocyte adhesion molecules in unstable coronary artery disease. Circulation. 1993;88(2):358-363.

46. Westhorpe CL, Dufour EM, Maisa A, Jaworowski A, Crowe SM, Muller WA. Endothelial cell activation promotes foam cell formation by monocytes following transendothelial migration in an in vitro model. Exp Mol Pathol. 2012;93(2):220-226.

47. de Groot PG, Urbanus RT, Roest M. Platelet interaction with the vessel wall. Handb Exp Pharmacol. 2012(210):87-110.

48. Ardigo D, Gaillard CA, Braam B. Application of leukocyte transcriptomes to assess systemic consequences of risk factors for cardiovascular disease. Clin Chem Lab Med. 2007;45(9):1109-1120.

49. Moore DF, Li H, Jeffries $\mathrm{N}$, et al. Using peripheral blood mononuclear cells to determine a gene expression profile of acute ischemic stroke: a pilot investigation. Circulation. 2005;111(2):212-221.

50. Patino WD, Mian OY, Kang JG, et al. Circulating transcriptome reveals markers of atherosclerosis. Proc Natl Acad Sci U S A. 2005;102(9):3423-3428. 
51. Ozaki Y, Imanishi T, Taruya A, et al. Circulating CD14+CD16+ monocyte subsets as biomarkers of the severity of coronary artery disease in patients with stable angina pectoris. Circ J. 2012;76(10):2412-2418.

52. Cheng XW, Kikuchi $\mathrm{R}$, Ishii $\mathrm{H}$, et al. Circulating cathepsin $\mathrm{K}$ as a potential novel biomarker of coronary artery disease. Atherosclerosis. 2013.

53. Schuck RN, Theken KN, Edin ML, et al. Cytochrome P450-derived eicosanoids and vascular dysfunction in coronary artery disease patients. Atherosclerosis. 2013;227(2):442-448.

54. Liuzzo G, Biasucci LM, Gallimore JR, et al. The prognostic value of C-reactive protein and serum amyloid a protein in severe unstable angina. $N$ Engl $\mathrm{J}$ Med. 1994;331(7):417-424.

55. Venet D, Dumont JE, Detours V. Most random gene expression signatures are significantly associated with breast cancer outcome. PLoS Comput Biol. 2011;7(10):e1002240. 



\section{Main findings in perspective}

The role of the monocyte/macrophage lineage has already been considered detrimental in atherosclerosis development for many years. Especially plaque macrophages have been studied extensively in relation to pathogenesis ${ }^{1}$. Thereby, the focus was not only on unraveling processes involved, but also on the determination of biomarkers for diagnosis and prognosis of cardiovascular disease (CVD) in general ${ }^{2,3}$. Over the last years, research has shifted its focus from plaque macrophages to circulating monocytes in relation to atherogenesis. In fact, it is acknowledged nowadays that these circulating monocytes might be as important for disease as the macrophages present in the atherosclerotic plaques ${ }^{4-7}$. Nevertheless, many functional aspects of circulating monocytes are not yet elucidated completely, e.g. the functional effects of hypercholesterolemia on circulating monocytes. Furthermore, these cells might yield so far unknown potential as biomarker, due to their monitoring capabilities of the vasculature.

This thesis focused on the role of circulating monocytes in atherosclerosis, and the question whether these cells exert effects locally in the lesion or systemically in the periphery. We set out to assess the effects of (I) depleting monocytes/macrophages from the circulation and lesion, as well as (II) the role of migration of circulating myeloid cells in relation to atherogenesis. Further, we studied (III) the functional effects of hypercholesterolemia on monocytes, and (IV) set out to gain more insight in the potential of circulating monocytes as biomarker for cardiovascular disease. The main findings of this thesis are:

1. Depletion of both $\mathrm{CD}_{11} 5^{+}$circulating monocytes and intimal macrophages attenuates plaque progression (Chapter $\mathbf{2}$ )

2. Increased monocyte chemotaxis towards the lesion, and thus augmented intimal macrophage numbers, reduces atherogenesis (Chapter 3)

3. Hypercholesterolemia induces excessive cholesterol accumulation and functional changes in circulating monocytes (Chapter 4)

4. Activated circulating monocytes harbor potential as (diagnostic and imaging) biomarkers in human atherosclerotic vessel disease (Chapter 5 and 6) 


\section{The effects of monocyte influx on plaque progression}

In Chapter 2, we investigated the effects of depleting both plaque-resident and systemic myeloid cells on atherosclerosis development. Here, a clear role for both plaque macrophages and circulating monocytes could be deduced from our results. In the first study we assessed the impact of whole body CD115 leukocyte ablation, as CD115 (or M-CSF receptor) is essential to monocyte/macrophage development. In contrast to our expectations, no effects on plaque development could be observed, which was only in part comparable to other studies on monocyte/macrophage ablation as reviewed by Croons et $a l^{8}$. These studies used genetic approaches in which human diphtheria toxin receptor (hDTR) expression was controlled by the myeloid specific promotor CD11b ${ }^{9,10}$, pharmacological ablation using clodronate liposomes ${ }^{11,12}$, and several other approaches. Even though the approaches differ substantially, the level of depletion of myeloid cells achieved in our study was at least comparable to these studies.

The difference between earlier studies and the study we performed was the combination of local apoptosis of plaque macrophages combined with systemic apoptosis of monocytes and granulocytes, but also osteoclasts. Calin et al already reported that depletion of monocytes and macrophages can act as a two-edged sword ${ }^{12}$. On the one hand, depletion of the monocyte/macrophage reduces the proteolytic and pro-inflammatory signals in the lesion. On the other hand, secondary necrosis accompanied by the release of cellular cholesterol and lipid deposits into the lesion contributes to plaque progression. Indeed, Döring and Soehnlein et al reported that interferon regulatory factor (IRF) 8 deficiency affects monocyte/macrophage function, resulting in defective intracellular lipid accumulation and disturbed efferocytosis. In combination with increased lesional neutrophil influx this led to accelerated atherosclerosis ${ }^{13}$.

Unexpectedly, CD115 leukocyte ablation affected hematopoiesis in which osteoclasts and bone marrow macrophages were depleted. This ablation resulted in uncontrolled release of immature leukocytes and progenitor cells from the bone marrow, as macrophages and osteoclasts are important regulators of hematopoiesis ${ }^{14-16}$. This contributed to the extramedullar myelopoiesis and monocytosis, in particular in spleen. Colony forming unit assays confirmed a shift in hematopoiesis from bone marrow to spleen. 
Overall, not only plaque monocytes/macrophages, but also peripheral ablation of $\mathrm{CD} 115^{+}$cells affected atherogenesis.

Furthermore, local induction of apoptosis in the atherosclerotic lesion resulted in plaque progression rather than attenuation of plaque development. Also here an important role for circulating monocytes could explain for the effects observed. Massive intimal macrophage apoptosis will lead to the buildup of inefficiently cleared apoptotic cellular debris ${ }^{17}$. Ineffective efferocytosis indeed was shown to result in secondary necrosis and increased necrotic core formation, which has major implications for atherosclerotic plaque progression 17-19. Consequently, a more pro-inflammatory, rather than anti-inflammatory milieu will develop resulting in the attraction of monocytes from the blood and enhanced plaque development ${ }^{20}$. In conclusion, our study identified an important contribution of $\mathrm{CD} 115^{+}$myeloid cells outside the atherosclerotic plaque affecting lesion progression. Nevertheless, localized modulation of monocytes/macrophages in the lesion could potentially be a valid target for therapeutic intervention as circulating monocytes do affect atherogenesis.

Next to ablation of monocytes/macrophages, the role of chemokine receptor desensitization was investigated in Chapter 3. In this study, we addressed the effects of hematopoietic GRK2 deficiency in atherosclerosis. GRK2 is a receptor kinase mediating desensitization and receptor recycling of G-protein coupled receptors for monocyte function and chemotaxis, such as CCR2 and CCR5 ${ }^{21,22}$. Inhibited desensitization of chemokine receptors in monocytes resulted in expanded plaque macrophage content, but decreased plaque size. Chemokine receptor signaling is a central process in atherogenesis ${ }^{23}$, but also in hematopoiesis, in particular for regulating stromal release of mature monocytes ${ }^{24}$. Indeed, inhibition of the CCL2, CX ${ }_{3} \mathrm{CR} 1$, or the CCL5/CCR5 axis results in dramatically reduced atherogenesis, indicative of the importance of both the chemokine receptor and ligands ${ }^{25}$. Besides involvement of GRK2 in chemokine receptor desensitization, the GRK2 interactome has been extended to endocytic proteins, but also non-receptor targets, e.g. growth arrest specific (Gas) $8^{\text {ref }}{ }^{26}$. GRK2 dysfunction is involved in several diseases, including cardiovascular pathologies (e.g. heart failure) ${ }^{27}$ and chronic inflammatory disease (e.g. rheumatoid arthritis) ${ }^{28}$. From the target receptors in combination with diseases with comparable pathogenesis, we deduced that GRK2 may also play an important role in atherosclerosis. 
Hematopoietic GRK2 deficiency led to enhanced monocyte migration, but attenuated plaque development. The role of circulating monocytes was inferred by excluding granulocytes and macrophages being responsible for the plaque phenotype. GRK2 deficiency resulted in increased proliferation as well as monocyte migration. Overall, increased monocyte chemotaxis resulted in plaque attenuation, most likely due to more efficient efferocytosis of apoptotic material, explaining the dramatic reduction in necrotic core size. These data show that stimulating monocyte chemotaxis towards the atherosclerotic lesion might be a promising target for intervention, especially in early atherosclerosis.

Hypercholesterolemia modifies circulating monocytes and their function

Next to inflammation-induced recruitment of monocytes towards the atherosclerotic lesion, hypercholesterolemia is the second hallmark of, and risk factor for, atherosclerosis ${ }^{29}$. Despite, the well-known association between hypercholesterolemia, especially increased LDL cholesterol levels, and the risk for cardiovascular disease, still little is known about the impact of hypercholesterolemia on circulating monocytes in the context of early atherosclerosis development. Most research focuses on the effects of long term (> 10 weeks) hypercholesterolemia on monocytes ${ }^{30,31}$. In our study we analyzed the short term $(<3$ weeks) effects on monocyte function upon Western type diet feeding and subsequent hypercholesterolemia.

In Chapter $\mathbf{4}$ we analyzed the early effects of diet-induced hypercholesterolemia on circulating monocyte subsets. Interestingly, and in contrast to the current paradigm, cholesterol does not only accumulate in plaque macrophages, but is also engulfed by circulating and splenic monocytes. Despite earlier observations of this phenomenon in murine and human monocytes by Mosig et al ${ }^{32,33}$ and Tolani et al ${ }^{34}$ the functional implications of this intracellular lipoprotein accumulation and its consequences for atherosclerosis development are not recognized completely.

Traditionally, LDL cholesterol is thought to preferentially accumulate in the arterial intima at places with reduced shear stress ${ }^{35}$. This process is followed by the influx of monocytes/macrophages, which scavenge the subendothelial oxLDL. We could now show that next to the cholesterol accumulation, monocyte-endothelial cell interaction is also enhanced in animals with increased cholesterol levels. As this increment was only present 
after activation of the endothelium by LPS stimulation, it is believed to reflect an intrinsically increased adhesive capacity of lipid-laden monocytes and this increased recruitment of monocytes to inflammatory vascular loci (e.g. atherosclerotic lesions). Nevertheless, hypercholesterolemia leads to endothelial cell dysfunction ${ }^{36}$, potentially contributing to increased monocyte rolling and adhesion. Our findings thus point out that cholesterol might also enter the atherosclerotic lesion via migration of lipid-laden circulating monocytes into the atherosclerotic lesion.

Intriguingly, our data indicate that hypercholesterolemia leads to a rapid monocytosis response, as well as an increase in plasma levels of proinflammatory cytokines within days after introduction of WTD. However, after three weeks of WTD plasma levels for the majority of cytokines appeared to normalize at least in part, suggesting that sustained hypercholesterolemia does not lead to prolonged hyper-inflammatory status. This indicates that hyperinflammation represents an early adaptive response to new metabolic conditions. In human disease, postprandial cholesterol and lipid levels are associated with cardiovascular events ${ }^{37,38}$. As we did not measure time points beyond three weeks or included reversal of diet to normal chow until now, it is still unclear to what extend cellular and systemic inflammatory and metabolic pathways affect systemic, or possibly local, inflammatory processes involved in early atherogenesis. Furthermore, the effects of these interventions on the regulation of cytokine levels in persistent hypercholesterolemia are to be determined. Recent evidence suggests that even mild reduction in cholesterol levels of hypercholesterolemic subjects can reduce plasma cytokine levels back to normal ${ }^{39}$. Nevertheless, more research on the interaction between cholesterol and cytokine levels is necessary to unravel the exact pathways and cell types responsible. 


\section{Circulating monocytes as biomarker for cardiovascular disease}

Monocytes and monocyte-derived proteins are still underrepresented amongst biomarkers for disease and this holds in particular for CVD, despite the critical roles exerted by this subset in its pathology, as we have shown in our murine atherosclerosis studies. As indicated in the introduction only a limited number of monocyte-derived biomarkers have been suggested thus far, such as CD163 and IL-6 secreted by peripheral blood mononuclear cells ${ }^{40,41}$. Besides these secreted proteins, non-secreted membrane markers also have been proposed to have diagnostic value, e.g. monocyte HLA-DR expression in acute liver failure ${ }^{42}$ and sialic acid-binding Ig-like lectin 1 (Siglec-1) and Fcy receptor I (CD64) expression in systemic lupus erythromatosus ${ }^{43,44}$. However, for cardiovascular disease, hardly any monocyte biomarkers have been described, apart from plakoglobin ${ }^{45}$. As monocytes play a critical role in the development of atherosclerosis and also have a patrolling function of the vasculature, they conceivably represent a rich and important source of diagnostic markers of disease status and prospect which legitimates more in depth searches for monocyte-derived biomarkers. In this thesis, we show that both on protein level (Chapter 5) and RNA level (Chapter 6) activated monocytes are encouraging sources of novel biomarkers for cardiovascular disease.

In Chapter $\mathbf{5}$ a whole cell phage display approach was applied for the discovery of novel membrane bound biomarkers on circulating monocytes from unstable angina pectoris patients. Although whole cell phage display is not a standardized technique, it has already been successfully applied for biomarker discovery ${ }^{46}$, especially in cancer research, where small tumor specimens were used as target ${ }^{47-50}$. However, also cell lines ${ }^{51}$ and isolated platelets ${ }^{52}$ have been considered as scaffold for phage display selections. Here, we applied whole cell phage display selection on LPS-activated THP-1 monocytes and validated the markers found on isolated $\mathrm{CD} 14^{+}$monocytes from unstable angina pectoris patients. To favor selection of relevant clones, we incorporated a depletion step using monocytes from healthy volunteers. Despite limited enrichment for clones binding UAP monocytes, monocytebased selections were proved to be efficient as we identified two clones with increased affinity for UAP monocytes. In addition, these clones showed their value for imaging, since they specifically bound monocytes in advanced, vulnerable atherosclerotic lesions, but not early lesions. Overall, from our study, monocytes emerge as a promising target for antibody discovery, in spite 
the large variety in surface proteins expressed on these cells. Our study also implies that circulating monocytes indeed can reflect local vascular processes such as atherosclerosis.

Inspired by these promising findings, we zoomed in on differences in RNA expression profiles between SAP, UAP, and healthy control $\mathrm{CD} 14^{+}$ monocytes in Chapter 6. Surprisingly, we could not detect any major differences between the study populations based on micro-array analysis. This is surprising as several groups have previously identified UAP-specific markers 53-55, albeit that none of these markers were cell-bound markers. These data suggest that secretome, rather than transcriptome, difference are present between patient populations. Still, Patel et al revealed that monocyte-platelet complexes did also not differ between SAP and UAP patients ${ }^{56}$ and differential expression of membrane receptors was also shown for UAP ${ }^{57,58}$. Even though our results did not show major differences in the transcriptome of UAP versus SAP monocytes, we are the first to investigate mRNA expression profiles of monocytes from SAP and UAP patients in a large patient cohort. In contrast to previous publications, we have studied differences in circulating CD $14^{+}$ monocytes in a patient cohort without any known cardiovascular disease, whereas others have studied total peripheral blood mononuclear cells ${ }^{59}$, specific patient populations (e.g. systemic lupus erythromatous patients ${ }^{57}$ ), or plaque macrophages ${ }^{60}$. Taken together, our study primarily focused on the discovery of diagnostic markers for discrimination between SAP and UAP patients, based on peripheral blood monocytes.

Interestingly, our studies on the proteome (chapter 5) and transciptome (chapter 6) do not accord completely. On the protein level we were able to identify altered surface expression on UAP monocytes compared to control monocytes using phage display. However, our phage display approach used LPS-activated THP-1 monocytes for target discovery and UAP monocytes for validation. This approach might have boosted the discrimination power of our phage display analysis. Nevertheless, LPS is a strong stimulus for TLR-signaling, which is not specific to UAP, but also is linked to various infectious and non-infectious diseases ${ }^{61}$. Preliminary data on microarray analysis from LPS-activated SAP monocytes with and without a CVD event during follow-up indicates that differences are present between the two study populations. However, the observation of differences within the 
population of SAP patients might also point to heterogeneity of SAP and UAP patients, contributing to the lack of separation between SAP and UAP patient monocyte transcriptomes. Still, the above suggests that activation potential, rather than basal expression levels, of UAP monocytes might be different from SAP monocytes. Indeed, first analysis of LPS activated monocytes from patients with event during follow-up compared to event-free controls, revealed an extensive list of differentially expressed genes. More in depth research on SAP and UAP patient monocytes using a broad 'omics' approach should reveal novel UAP specific monocytic biomarkers useful for imaging, diagnosis, and potentially even risk prediction.

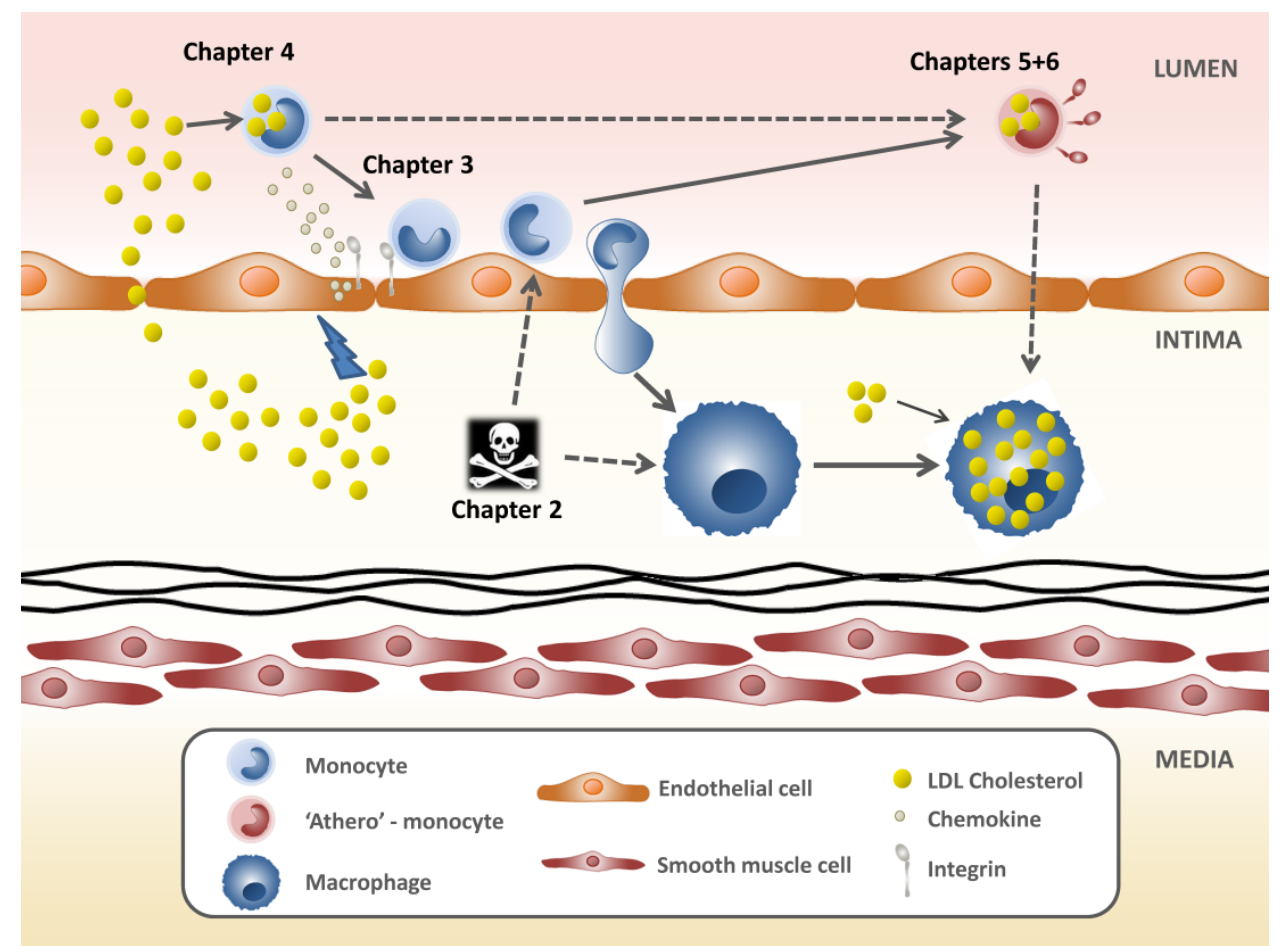

Figure 1 - Overview of the role of circulating monocytes in atherosclerosis

Circulating monocytes contribute to atherosclerosis development at different levels. In Chapter 2 we showed the role of circulating monocytes in combination with plaque macrophages in regulating plaque progression, by induction of $\mathrm{CD} 115^{+}$cell apoptosis. Regulation of circulating monocyte chemotaxis via GRK2 towards the atherosclerotic lesion was discussed in Chapter $\mathbf{3}$. Interaction of circulating monocytes with cholesterol and the atherosclerotic lesion as well as the effects thereof on proteome and transcriptome biomarkers were discussed in Chapter $\mathbf{4}$ and Chapters 5 and 6, respectively. 


\section{Concluding Remarks and Future Perspectives}

The role of circulating monocytes in atherosclerosis was investigated in this thesis. We were able to show that monocyte migration as well as monocyte/macrophage apoptosis are indeed essential processes in atherogenesis. Next, hypercholesterolemia was proven to influence circulating monocyte function rapidly after onset hypercholesterolemia. Finally, we have explored circulating monocytes as a novel target for current, but also future biomarker research. Here, we identified activated monocytes as a potential marker for diagnosis and imaging of patients at risk for CVD, both on protein and RNA level. Taken together, monocytes not only appear to play a central role in regulating plaque progression, but are also useful biomarkers for diagnosis, imaging, and possibly even prognosis of cardiovascular disease.

Circulating monocytes are thus central actors in atherogenesis. We were able to identify these cells not only being essential for plaque initiation and progression, but also pinpoint novel opportunities for CVD biomarkers. Nevertheless, additional research on activation potential of monocytes will contribute to both unraveling mechanism involved in monocyte function and additional information on current and future disease and risk for cardiovascular events, such as myocardial infarction and stroke. Future research will need to focus on more in depth knowledge regarding the translation from local information in the atherosclerotic lesion to the circulating monocytes, to determine additional markers for diagnosis, risk prediction, and imaging. Further, screening methods which can reliably and successfully identify these subtle yet important differences need to be optimized facilitating minimally invasive and rapid measurements of these circulatory biomarkers. 


\section{Circulating monocytes in atherosclerosis: Local or systemic actors?}

The central question of this thesis was whether circulating monocytes only impact atherosclerosis at a local level or also systemically. Our data indicate that indeed both peripheral and local monocyte functions are relevant for atherosclerosis development. Locally, monocyte chemotaxis and monocyte/macrophage ablation influence plaque development as we have shown. Lipid-loading of circulating monocytes not only could contribute to cholesterol accumulation in the lesions, but also alter monocyte function systemically. Nevertheless, influencing monocytes systemically will not only affect plaque development, but also monocyte functionality (e.g. patrolling behavior). In the end circulating monocytes have the potential to be used as novel biomarker for CVD, as we have shown in this thesis, indicating the link between local vascular pathology and systemic effects on monocytes. 


\section{References}

1. Moore KJ, Tabas I. Macrophages in the pathogenesis of atherosclerosis. Cell. 2011;145(3):341-355.

2. Moller MJ, Qin Z, Toursarkissian B. Tissue markers in human atherosclerotic carotid artery plaque. Ann Vasc Surg. 2012;26(8):1160-1165.

3. Morishige $\mathrm{K}$, Kacher DF, Libby $\mathrm{P}$, et al. High-resolution magnetic resonance imaging enhanced with superparamagnetic nanoparticles measures macrophage burden in atherosclerosis. Circulation. 2010;122(17):1707-1715.

4. Warmington KS, Boring L, Ruth JH, et al. Effect of C-C chemokine receptor 2 (CCR2) knockout on type-2 (schistosomal antigen-elicited) pulmonary granuloma formation: analysis of cellular recruitment and cytokine responses. Am J Pathol. 1999;154(5):1407-1416.

5. Hristov M, Leyendecker T, Schuhmann C, et al. Circulating monocyte subsets and cardiovascular risk factors in coronary artery disease. Thromb Haemost. 2010;104(2):412-414.

6. Auffray C, Sieweke MH, Geissmann F. Blood monocytes: development, heterogeneity, and relationship with dendritic cells. Annu Rev Immunol. 2009;27:669692.

7. Swirski FK, Weissleder R, Pittet MJ. Heterogeneous in vivo behavior of monocyte subsets in atherosclerosis. Arterioscler Thromb Vasc Biol. 2009;29(10):14241432.

8. Croons V, Martinet W, De Meyer GR. Selective removal of macrophages in atherosclerotic plaques as a pharmacological approach for plaque stabilization: benefits versus potential complications. Curr Vasc Pharmacol. 2010;8(4):495-508.

9. Duffield JS, Forbes SJ, Constandinou CM, et al. Selective depletion of macrophages reveals distinct, opposing roles during liver injury and repair. J Clin Invest. 2005;115(1):56-65.

10. Stoneman V, Braganza D, Figg N, et al. Monocyte/macrophage suppression in $\mathrm{CD} 11 \mathrm{~b}$ diphtheria toxin receptor transgenic mice differentially affects atherogenesis and established plaques. Circ Res. 2007;100(6):884-893.

11. Van Rooijen N, Sanders A. Liposome mediated depletion of macrophages: mechanism of action, preparation of liposomes and applications. J Immunol Methods. 1994;174(1-2):83-93.

12. Calin MV, Manduteanu I, Dragomir E, et al. Effect of depletion of monocytes/macrophages on early aortic valve lesion in experimental hyperlipidemia. Cell Tissue Res. 2009;336(2):237-248.

13. Doring $\mathrm{Y}$, Soehnlein $\mathrm{O}$, Drechsler $\mathrm{M}$, et al. Hematopoietic interferon regulatory factor 8-deficiency accelerates atherosclerosis in mice. Arterioscler Thromb Vasc Biol. 2012;32(7):1613-1623.

14. Wu Y, Madri J. Insights into monocyte-driven osteoclastogenesis and its link with hematopoiesis: regulatory roles of PECAM-1 (CD31) and SHP-1. Crit Rev Immunol. 2010;30(5):423-433. 
15. Cecchini MG, Hofstetter W, Halasy J, Wetterwald A, Felix R. Role of CSF-1 in bone and bone marrow development. Mol Reprod Dev. 1997;46(1):75-83; discussion 83-74.

16. Winkler IG, Sims NA, Pettit AR, et al. Bone marrow macrophages maintain hematopoietic stem cell (HSC) niches and their depletion mobilizes HSCs. Blood. 2010;116(23):4815-4828.

17. Van Vre EA, Ait-Oufella H, Tedgui A, Mallat Z. Apoptotic cell death and efferocytosis in atherosclerosis. Arterioscler Thromb Vasc Biol. 2012;32(4):887-893.

18. Liao X, Sluimer JC, Wang Y, et al. Macrophage autophagy plays a protective role in advanced atherosclerosis. Cell Metab. 2012;15(4):545-553.

19. Thorp E, Subramanian M, Tabas I. The role of macrophages and dendritic cells in the clearance of apoptotic cells in advanced atherosclerosis. Eur J Immunol. 2011;41(9):2515-2518.

20. Thorp EB. Contrasting Inflammation Resolution during Atherosclerosis and Post Myocardial Infarction at the Level of Monocyte/Macrophage Phagocytic Clearance. Front Immunol. 2012;3:39.

21. Aragay AM, Mellado M, Frade JM, et al. Monocyte chemoattractant protein-1induced CCR2B receptor desensitization mediated by the $G$ protein-coupled receptor kinase 2. Proc Natl Acad Sci U S A. 1998;95(6):2985-2990.

22. Oppermann M, Mack M, Proudfoot AE, Olbrich H. Differential effects of CC chemokines on CC chemokine receptor 5 (CCR5) phosphorylation and identification of phosphorylation sites on the CCR5 carboxyl terminus. J Biol Chem. 1999;274(13):88758885 .

23. Weber C, Schober A, Zernecke A. Chemokines: key regulators of mononuclear cell recruitment in atherosclerotic vascular disease. Arterioscler Thromb Vasc Biol. 2004;24(11):1997-2008.

24. Serbina NV, Pamer EG. Monocyte emigration from bone marrow during bacterial infection requires signals mediated by chemokine receptor CCR2. Nat Immunol. 2006;7(3):311-317.

25. Combadiere $C$, Potteaux $S$, Rodero $M$, et al. Combined inhibition of $C C L 2$, CX3CR1, and CCR5 abrogates Ly6C(hi) and Ly6C(lo) monocytosis and almost abolishes atherosclerosis in hypercholesterolemic mice. Circulation. 2008;117(13):1649-1657.

26. Evron T, Daigle TL, Caron MG. GRK2: multiple roles beyond G protein-coupled receptor desensitization. Trends Pharmacol Sci. 2012;33(3):154-164.

27. Raake PW, Vinge LE, Gao E, et al. G protein-coupled receptor kinase 2 ablation in cardiac myocytes before or after myocardial infarction prevents heart failure. Circ Res. 2008;103(4):413-422.

28. Lombardi MS, Kavelaars A, Schedlowski M, et al. Decreased expression and activity of G-protein-coupled receptor kinases in peripheral blood mononuclear cells of patients with rheumatoid arthritis. FASEB J. 1999;13(6):715-725.

29. Halvorsen B, Otterdal K, Dahl TB, et al. Atherosclerotic plaque stability--what determines the fate of a plaque? Prog Cardiovasc Dis. 2008;51(3):183-194. 
30. Swirski FK, Libby P, Aikawa E, et al. Ly-6Chi monocytes dominate hypercholesterolemia-associated monocytosis and give rise to macrophages in atheromata. J Clin Invest. 2007;117(1):195-205.

31. Murphy AJ, Akhtari M, Tolani S, et al. ApoE regulates hematopoietic stem cell proliferation, monocytosis, and monocyte accumulation in atherosclerotic lesions in mice. J Clin Invest. 2011;121(10):4138-4149.

32. Mosig S, Rennert K, Buttner $\mathrm{P}$, et al. Monocytes of patients with familial hypercholesterolemia show alterations in cholesterol metabolism. BMC Med Genomics. 2008;1:60.

33. Mosig S, Rennert K, Krause S, et al. Different functions of monocyte subsets in familial hypercholesterolemia: potential function of CD14+ CD16+ monocytes in detoxification of oxidized LDL. FASEB J. 2009;23(3):866-874.

34. Tolani S, Pagler TA, Murphy AJ, et al. Hypercholesterolemia and reduced HDL$\mathrm{C}$ promote hematopoietic stem cell proliferation and monocytosis: Studies in mice and FH children. Atherosclerosis. 2013.

35. Zernecke A, Weber C. Improving the treatment of atherosclerosis by linking anti-inflammatory and lipid modulating strategies. Heart. 2012;98(21):1600-1606.

36. Li D, Zhang $\mathrm{Y}, \mathrm{Ma} \mathrm{J}$, Ling $\mathrm{W}$, Xia M. Adenosine monophosphate activated protein kinase regulates $A B C G 1$-mediated oxysterol efflux from endothelial cells and protects against hypercholesterolemia-induced endothelial dysfunction. Arterioscler Thromb Vasc Biol. 2010;30(7):1354-1362.

37. Boccalandro F, Farias J, Boccalandro C, Vaisman D. Frequency of postprandial lipemia after a first acute coronary event (unstable angina pectoris or non-ST-segment elevation acute myocardial infarction) and the effects of atenolol on the lipemia. Am J Cardiol. 2002;90(2):153-156.

38. Nordestgaard BG, Langsted A, Freiberg JJ. Nonfasting hyperlipidemia and cardiovascular disease. Curr Drug Targets. 2009;10(4):328-335.

39. Krysiak R, Okopien B. Different effects of simvastatin on ex vivo monocyte cytokine release in patients with hypercholesterolemia and impaired glucose tolerance. J Physiol Pharmacol. 2010;61(6):725-732.

40. Bielecki M, Kowal K, Lapinska A, Chyczewski L, Kowal-Bielecka O. Increased release of soluble CD163 by the peripheral blood mononuclear cells is associated with worse prognosis in patients with systemic sclerosis. Adv Med Sci. 2013:13-20.

41. Kaplin A, Carroll KA, Cheng J, et al. IL-6 release by LPS-stimulated peripheral blood mononuclear cells as a potential biomarker in Alzheimer's disease. Int Psychogeriatr. 2009;21(2):413-414.

42. Antoniades CG, Berry PA, Davies ET, et al. Reduced monocyte HLA-DR expression: a novel biomarker of disease severity and outcome in acetaminopheninduced acute liver failure. Hepatology. 2006;44(1):34-43.

43. Li Y, Lee PY, Kellner ES, et al. Monocyte surface expression of Fcgamma receptor RI (CD64), a biomarker reflecting type-I interferon levels in systemic lupus erythematosus. Arthritis Res Ther. 2010;12(3):R90. 
44. Biesen R, Demir C, Barkhudarova F, et al. Sialic acid-binding Ig-like lectin 1 expression in inflammatory and resident monocytes is a potential biomarker for monitoring disease activity and success of therapy in systemic lupus erythematosus. Arthritis Rheum. 2008;58(4):1136-1145.

45. Cooksley-Decasper S, Reiser H, Thommen DS, et al. Antibody phage display assisted identification of junction plakoglobin as a potential biomarker for atherosclerosis. PLoS One. 2012;7(10):e47985.

46. Watters JM, Telleman P, Junghans RP. An optimized method for cell-based phage display panning. Immunotechnology. 1997;3(1):21-29.

47. Baral TN, Murad Y, Nguyen TD, Iqbal U, Zhang J. Isolation of functional single domain antibody by whole cell immunization: implications for cancer treatment. J Immunol Methods. 2011;371(1-2):70-80.

48. Shukla GS, Krag DN. Selection of tumor-targeting agents on freshly excised human breast tumors using a phage display library. Oncol Rep. 2005;13(4):757-764.

49. Shukla GS, Krag DN. Phage display selection for cell-specific ligands: development of a screening procedure suitable for small tumor specimens. J Drug Target. 2005;13(1):7-18.

50. Popkov M, Rader C, Barbas CF, 3rd. Isolation of human prostate cancer cell reactive antibodies using phage display technology. J Immunol Methods. 2004;291(12):137-151.

51. Cao J, Zhao P, Miao XH, Zhao L, Xue L, Qi Zt Z. Phage display selection on whole cells yields a small peptide specific for HCV receptor human CD81. Cell Res. 2003;13(6):473-479.

52. Hagay $\mathrm{Y}$, Lahav J, Levanon A, Panet A. Function-modulating human monoclonal antibodies against platelet-membrane receptors isolated from a phagedisplay library. J Thromb Haemost. 2003;1(8):1829-1836.

53. Lu HQ, Liang C, He ZQ, Fan M, Wu ZG. Circulating miR-214 is associated with the severity of coronary artery disease. J Geriatr Cardiol. 2013;10(1):34-38.

54. Fujibayashi K, Kawai Y, Kitayama M, et al. Serum deoxyribonuclease I activity can be a useful diagnostic marker for the early diagnosis of unstable angina pectoris or non-ST-segment elevation myocardial infarction. J Cardiol. 2012;59(3):258-265.

55. Momiyama $\mathrm{Y}$, Ohmori R, Tanaka N, et al. High plasma levels of matrix metalloproteinase-8 in patients with unstable angina. Atherosclerosis. 2010;209(1):206-210.

56. Patel PB, Pfau SE, Cleman MW, et al. Comparison of coronary artery specific leukocyte-platelet conjugate formation in unstable versus stable angina pectoris. $\mathrm{Am} \mathrm{J}$ Cardiol. 2004;93(4):410-413.

57. Raaz-Schrauder D, Ekici AB, Munoz LE, et al. Patients with unstable angina pectoris show an increased frequency of the Fc gamma RIla R131 allele. Autoimmunity. 2012;45(7):556-564.

58. Ikejima $H$, Imanishi $T$, Tsujioka $H$, et al. Upregulation of fractalkine and its receptor, CX3CR1, is associated with coronary plaque rupture in patients with unstable angina pectoris. Circ J. 2010;74(2):337-345. 
59. Li HL, Peng WH, Cui ST, et al. Vaspin plasma concentrations and mRNA expressions in patients with stable and unstable angina pectoris. Clin Chem Lab Med. 2011;49(9):1547-1554.

60. Adachi T, Naruko T, Itoh A, et al. Neopterin is associated with plaque inflammation and destabilisation in human coronary atherosclerotic lesions. Heart. 2007;93(12):1537-1541.

61. Noreen M, Shah MA, Mall SM, et al. TLR4 polymorphisms and disease susceptibility. Inflamm Res. 2012;61(3):177-188. 

$\because \because c^{\prime}$

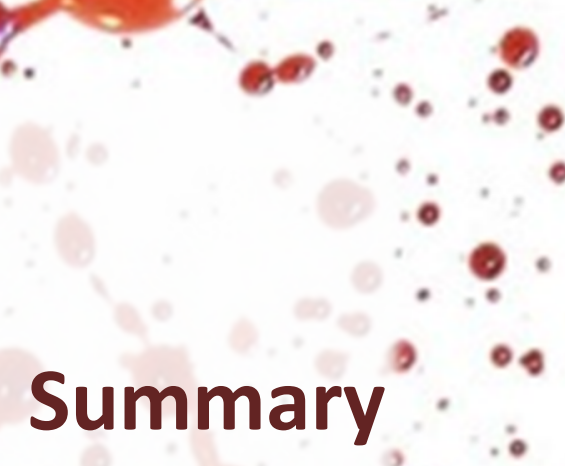

86

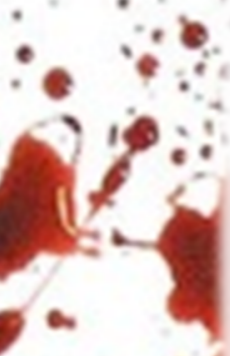

8

$e^{2}$

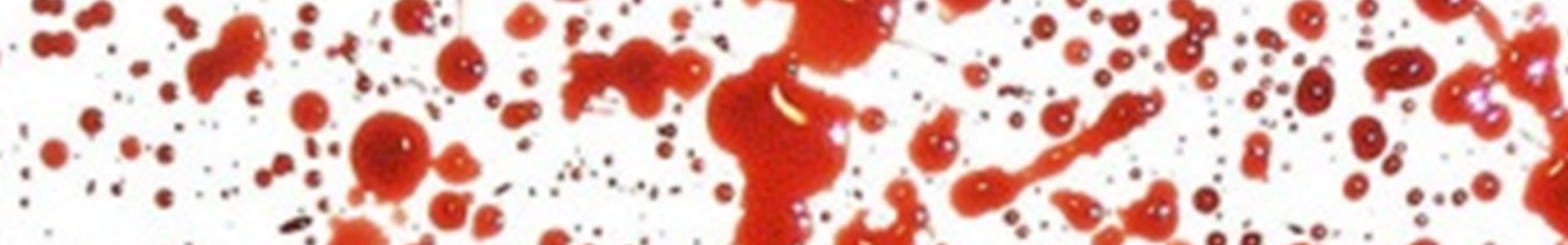
$\therefore$ *

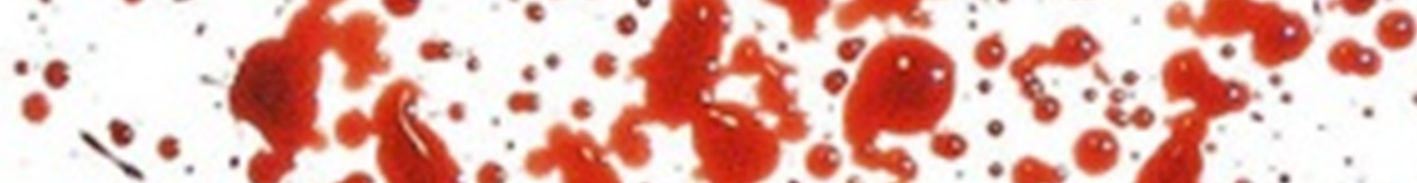

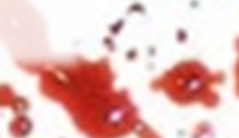


Cardiovascular diseases are still one of the leading causes of death in the Western societies and account for over 17 million deaths world-wide annually. Atherosclerosis is the predominant underlying pathology responsible for various cardiovascular diseases.

Atherosclerosis is characterized by the accumulation of low density lipoprotein (LDL) cholesterol in the intima of arteries. Oxidation of intimal cholesterol will result in activation of the vessel wall and will trigger a proinflammatory cascade. Due to the release of chemokines and upregulation of integrins on the endothelial cells covering the early lesion leukocytes will be attracted in an effort to resolve the immune response and remove the cholesterol. In the first stage, predominantly monocytes will migrate into the early atherosclerotic lesion, and give rise to plaque macrophages which will sequester the local oxidized cholesterol. Excessive intra-cellular cholesterol accumulation combined with ineffective excretion results cholesterol filled foamy macrophages also known as foam cells. Apoptosis of foam cells combined with ineffective clearance of apoptotic cells, will result in buildup of cell debris and (oxidized) cholesterol in the lesion, forming a lipid-rich, necrotic core, covered by a fibrous cap of smooth muscle cells and collagen. Destabilization of the fibrous cap by proteases secreted by macrophages and other inflammatory cells in the lesion, can eventually result in plaque rupture, exposing the pro-thrombotic content of the lesion to the circulation, potentially resulting in clinical complications, such as myocardial infarction and stroke.

Monocytes and plaque macrophages are instrumental in the development of atherosclerosis, from initial early until advanced vulnerable lesion. The role of circulating monocytes in relation to atherosclerosis development is not completely elucidated, despite the importance of these subsets in the development of the disease. Therefore, this thesis investigated the role of circulating monocytes in atherosclerosis, to determine (I) whether apart from plaque residing monocytic cell pools, the peripheral monocytes are able to impact disease onset and progression, and (II) whether these cells have potential as biomarker for cardiovascular disease in humans.

The function of circulating monocytes as well as plaque macrophages can be studied by specific depletion of these cell types. In Chapter 2, we studied the impact of systemic versus plaque-targeted ablation of myeloid cells on atherosclerosis in $\mathrm{ApoE}^{-/}$mice, bearing a Macrophage FAS Induced 
Apoptosis (MaFIA) suicide gene. Local ablation of CD115 positive plaque macrophages aggravated atherosclerosis, as both necrotic core and plaque size were expanded significantly by promoting either clonal expansion of plaque macrophages or monocyte influx. In contrast, systemic ablation of CD115 positive myeloid cells did not result in changes in plaque burden but promoted a more vulnerable plaque phenotype. Interestingly, it was associated with enhanced extramedullar hematopoiesis and mobilization of immature myeloid cells into the circulation. Our data suggest that the augmented extra-medullar myelopoiesis and the increased mobilization of immature myeloid cells into the circulation, upon systemic induction of monocyte/macrophage apoptosis may have underlain the observed increase in plaque vulnerability.

As migration of leukocytes towards the lesion is a key process in atherogenesis, we describe, in Chapter 3, the role of chemotaxis regulation on atherosclerosis development. Chemokine receptors are $\mathrm{G}$ protein-coupled receptors (GPCR) and are responsible for the directional migration of cells along a chemokine gradient. The activity of GPCRs is controlled by receptor kinases, such as GRK2. We here studied the effect of partial hematopoietic GRK2 deficiency on atherosclerosis development in LDL receptor knock-out mice. Hematopoietic $\mathrm{GRK}^{+/-}$deficiency resulted in marked attenuation of plaque development. To exclude that macrophage and granulocyte GRK2 was responsible for the effects observed, we used a LysM-cre GRK2 $2^{\text {flox/flox }}$ conditional knock-out model which results in macrophage and granulocyte specific GRK2 deficiency. As atherosclerosis development was not affected in this model, we concluded that monocytes were most likely responsible for the phenotype observed in the $\mathrm{GRK}^{+/}$study and that even partial GRK2 deficiency already was able to prevent atherosclerotic lesion development beyond the fatty streak stage.

Next to inflammation, hypercholesterolemia is one of the key processes in atherosclerosis development and a well-known risk factor for cardiovascular disease, and evidence is culminating that both actors are tightly associated. In Chapter 4, we analyzed the interaction between plasma cholesterol and circulating monocytes in LDL receptor deficient mice to unravel the effects of hypercholesterolemia on monocyte function and numbers in early atherosclerosis. We could not only show that under hyperlipidemic conditions, circulating monocytes carry lipoprotein depositions already before entering the lesion, and were able to link this to alterations in monocyte 
function. In fact, cholesterol-laden monocytes exhibited increased reactive oxygen species production and rolling along and adhesion to activated endothelium. Combined with the inflammatory cytokine profile observed in these animals, hypercholesterolemia was shown to affect circulating monocytes already in very early phases of atherosclerosis development and might reveal novel pathways contributing to intimal cholesterol accumulation.

As circulating monocyte subsets have a patrolling function in the vasculature we studied the biomarker potential of human circulating monocytes. We analyzed the clinical perspective of using circulating monocytes for disease detection, as well as therapy, both on a proteome and transcriptome level.

In Chapter 5, we identified at least two markers which may yield potential as diagnostic biomarker in unstable angina pectoris, as well as in molecular imaging approaches. Phage display affinity selections were performed on activated THP-1 monocytes to identify clones of interest. Subsequently, these selected clones were validated on CD14 positive monocytes isolated from healthy controls and unstable angina pectoris patients, as well as on early stable and advanced vulnerable plaque tissue, identifying at least two clones with high specificity.

In Chapter 6, we analyzed the transcriptome of monocytes isolated from stable and unstable angina pectoris patients to identify biomarkers specific for unstable angina pectoris. The patient populations did not differ in terms of monocyte markers or chemokine receptor expression as determined by flow cytometry. Surprisingly, we could also not detect any major differences in the transcriptome of these monocytes between stable and unstable angina pectoris, despite major differences in clinical parameters, and as reported plasma cytokine patterns. These data indicate that at baseline mild acute cardiovascular syndromes such as unstable angina pectoris do not impact the transcriptome of circulating monocytes.

Finally, in Chapter $\mathbf{7}$ the findings presented in this thesis are discussed and future perspectives are indicated. In conclusion, we were able to confirm that monocytes play an important role in atherosclerotic lesion development. Monocyte migration towards the lesion as well as numbers and function of plaque macrophages were shown to be critical in atherogenesis, but also the interaction between plasma cholesterol and circulating monocytes was identified to have importance in disease development. Furthermore, 
circulating monocytes convey information from local sites, such as atherosclerotic plaques, to the periphery. Indeed, we were able to show that circulating monocytes harbor potential as diagnostic biomarker for at least some cardiovascular pathologies. Future research will need to focus on identification of specific targets as well as on investigation of the prospective of activation potential of circulating monocytes in relation to biomarker discovery. 



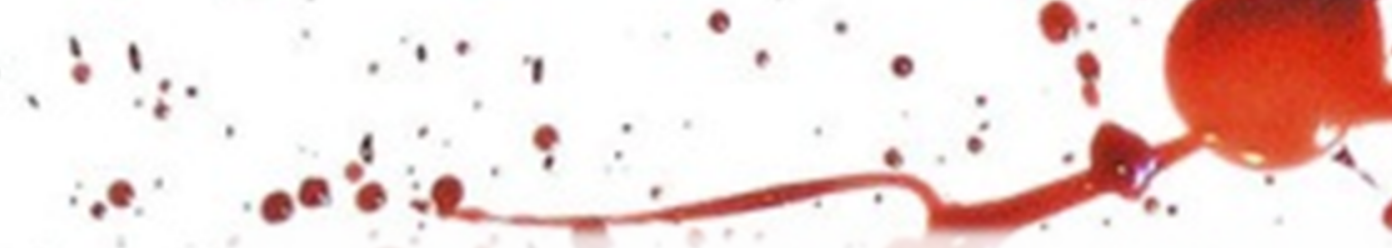


Hart- en vaatziekten zijn nog altijd een van de meest voorkomende doodsoorzaken in de Westerse landen en zijn verantwoordelijk voor meer dan 17 miljoen doden per jaar wereldwijd. Aderverkalking, ook wel atherosclerose genoemd, is één van de belangrijkste onderliggende oorzaken verantwoordelijk voor de gevolgen van hart- en vaatziekten, zoals hersen- en hartinfarct.

Atherosclerose is een ziekte van de middelgrote en grote slagaders, waarbij cholesterol zich opstapelt in de vaatwand door een ontregeling van de cellen die de vaatwand bedekken op plaatsen waar de bloedstroom verstoord is, zoals bochten en vertakkingen van de vaatboom. Door de ophoping van cholesterol ontstaat er een ontstekingsreactie in de vaatwand die ervoor zorgt dat witte bloedcellen worden aangetrokken. In eerste instantie zullen vooral monocyten, dit is een type witte bloedcel, naar de vaatwand migreren. In de vaatwand zullen deze cellen zich verder ontwikkelen en vormen ze zogenaamde macrofagen, die het cholesterol dat aanwezig is in de vaatwand zullen gaan opnemen. Door de grote hoeveelheid cholesterol die zich ophoopt in de cellen zullen deze een schuimachtig uiterlijk krijgen, waardoor ze schuimcel genoemd worden. Als deze schuimcellen te veel cholesterol hebben opgenomen, zullen ze dood gaan en zal het opgenomen cholesterol, maar ook de resten van de gestorven cellen achterblijven in de vaatwand. Er ontstaat hierdoor uiteindelijk een cholesterol-rijke kern die middels een kapsel bestaande uit collageen en gladde spiercellen wordt afgescheiden van het bloed: de atherosclerotische plaque. Afbraak van het kapsel dat over de plaque ligt, kan resulteren in het scheuren van het kapsel, waardoor het protrombotische materiaal in de plaque wordt blootgesteld aan het bloed en er een bloedstolsel zal ontstaan. Het is dit bloedstolsel dat door afsluiting van het bloedvat kan leiden tot zuurstofgebrek in het achterliggende weefsel, waardoor een hart- of herseninfarct ontstaat.

Monocyten in het bloed en macrofagen in de plaque zijn belangrijke cellen in de ontwikkeling van atherosclerose, zowel in beginnende plaques als ook in meer gevorderde stadia van het ziekteproces. De rol van monocyten in het bloed in relatie tot de ontwikkeling van atherosclerose is nog niet volledig opgehelderd, ondanks dat deze cellen worden gezien als een zeer belangrijk celtype tijdens de ontwikkeling van deze ziekte. Mijn onderzoek richtte zich op de rol van deze in het bloed circulerende monocyten in de ontwikkeling van atherosclerose, waarbij ik getracht heb om te achterhalen hoe deze cellen de 
onderliggende processen beïnvloeden die ten grondslag liggen aan atherosclerose. Het uiteindelijke doel van dit onderzoek was te bepalen of deze cellen gebruikt kunnen worden als biomarker voor hart- en vaatziekten.

De functie van circulerende monocyten, maar ook macrofagen in de plaque, kan effectief worden bestudeerd door deze cellen te elimineren. In Hoofdstuk 2 hebben we aangetoond dat circulerende monocyten plaque ontwikkeling kunnen afremmen in een muismodel waarin bepaalde witte bloedcellen, zoals monocyten en macrofagen, na toediening van een chemische stof, verwijderd worden. Eliminatie van zowel plaque macrofagen alsook circulerende monocyten resulteerde niet in veranderingen in plaque ontwikkeling vergeleken met de controle. Wel werd er een verhoogde productie van witte bloedcellen (hematopoëse) waargenomen, waarbij er mogelijk sprake was van een toegenomen migratie van monocyten naar de plaque. Als alleen de plaque macrofagen werden verwijderd, resulteerde dit in een toename van de plaque ontwikkeling. Samenvattend hebben we kunnen aantonen dat plaque ontwikkeling beïnvloed kan worden door de beschikbaarheid van circulerende monocyten alsook de aanwezigheid van macrofagen in de plaque, waarbij de verstoorde productie van deze cellen een belangrijke rol speelt.

In Hoofdstuk 3 beschrijven we de rol van gerichte migratie van cellen naar een stimulus, en de regulatie van dit proces in atherosclerose. Chemokine receptoren zijn verantwoordelijk voor deze migratie van cellen en ze behoren tot de familie van $\mathrm{G}$ eiwit gekoppelde receptoren. De activiteit van deze receptoren wordt gereguleerd door bepaalde enzymen, zoals GRK2. We hebben het effect van gedeeltelijke uitschakeling van GRK2 in witte bloedcellen onderzocht in pro-atherogene knock-out muizen. Gedeeltelijke uitschakeling van GRK2 resulteerde in zeer sterke remming van atherosclerose ontwikkeling. Uitschakeling van dit enzym in granulocyten en macrofagen, naast monocyten zijn dit andere witte bloedcellen die GRK2 aanmaken, liet geen effect zien op de ontwikkeling van atherosclerose, wat suggereert dat GRK2 in (circulerende) monocyten verantwoordelijk is voor de remming van atherosclerose.

Naast ontstekingsreacties is ook een verhoogd cholesterol niveau betrokken bij de ontwikkeling van atherosclerose en een risicofactor voor de ontwikkeling van hart- en vaatziekten. In Hoofdstuk $\mathbf{4}$ hebben we de interactie tussen cholesterol in het bloed en circulerende monocyten onderzocht. We 
konden aantonen dat naast het in de plaque opgehoopte cholesterol ook het in het bloed aanwezige cholesterol van belang is voor de ontwikkeling van atherosclerose, omdat dit wordt opgenomen door circulerende monocyten. Hierbij worden bepaalde functies van deze monocyten ook beïnvloed. Cholesterol-geladen monocyten produceren meer zuurstofradicalen en hechten nog beter dan normaal aan de ontstoken bloedvatwand. Samen met een verhoogde concentratie van bepaalde ontstekingsmediatoren in het bloed, die worden waargenomen na het volgen van een cholesterol/vetrijk dieet dragen deze veranderingen in de monocyten bij aan het ontstaan van atherosclerose. De toegenomen hechting van deze ontstekingscellen aan de vaatwand in combinatie met de ophoping van cholesterol in deze circulerende monocyten, zijn een nieuwe route waarlangs cholesterol vanuit het bloed in de vaatwand terecht kan komen, waardoor uiteindelijk een atherosclerotische plaque zal ontstaan.

Aangezien circulerende monocyten ook een functie hebben als verkenners van de bloedvaten, hebben we bestudeerd of deze cellen potentie hebben als marker voor hart- en vaatziekten in de mens. We hebben zowel het eiwit als het DNA niveau bestudeerd om deze nieuwe markers te vinden. In Hoofdstuk 5 hebben we minstens twee markers kunnen identificeren die potentie hebben als diagnostische marker voor instabiele angina, oftewel pijn op de borst in rust, maar daarnaast ook mogelijkheden bieden om gebruikt te worden bij detectiemethodes die gebruik maken van beeldtechnieken. Bindende antilichamen werden geselecteerd met behulp van faag display selecties op geactiveerde humane monocyten (de THP-1 cellijn). Bij faag display worden antilichamen gekoppeld aan virusdeeltjes, waarmee de antilichamen die binden aan de monocyten geselecteerd kunnen worden door deze te laten reageren met de monocyten en niet-bindende antilichamen weg te wassen. Hierna werden de geselecteerde antilichamen getest op hun capaciteit om te binden aan monocyten geïsoleerd uit patiënten met instabiele angina, maar ook aan monocyten in vroege en meer gevorderde atherosclerotische plaques. Minstens twee antilichamen bleken met hoge voorkeur te binden aan deze cellen en zijn mogelijk geschikt voor de diagnose van hart- en vaatziekten en mogelijk zelfs voor het opsporen van gevorderde plaques. 
In Hoofdstuk 6 hebben we getracht om biomarkers te identificeren waarmee onderscheid gemaakt kan worden tussen stabiele angina (allen bij inspanning pijn-op-de-borst) en instabiele angina (ook tijdens rust pijn-op-deborst) patiënten. Allereerst hebben we gekeken of er verschillen aanwezig waren tussen de hoeveelheid eiwitten die aanwezig zijn op de buitenkant van de monocyten. We konden aantonen dat de monocyten van deze twee patiëntgroepen hierin niet van elkaar verschilden. Vervolgens hebben we gekeken naar de mate waarin informatie van het DNA door de cel gebruikt wordt om eiwitten te produceren en of hierin verschillen aanwezig waren tussen de patiëntgroepen. Tegen onze verwachtingen in konden we ook voor deze eigenschappen geen verschillen ontdekken tussen monocyten van stabiele angina patiënten en instabiele angina patiënten. Onze studie toont daarmee aan dat er geen grote verschillen aanwezig zijn in circulerende monocyten van stabiele en instabiele angina patiënten.

Tot slot worden in Hoofdstuk $\mathbf{7}$ de resultaten, gepresenteerd in dit proefschrift, bediscussieerd en worden toekomstige mogelijkheden voor onderzoek aangegeven. Samenvattend toont dit onderzoek aan dat circulerende monocyten essentieel zijn voor de ontwikkeling van atherosclerose. We hebben kunnen aantonen dat de migratie van monocyten naar de plaque, maar ook de hoeveelheid macrofagen in de plaque kritieke processen zijn in de ontwikkeling van atherosclerose. Daarnaast tonen onze studies aan dat cholesterol al in de bloedsomloop de functie van de circulerende monocyten ongunstig beïnvloedt, een tot nu onderbelicht mechanisme bij het ontstaan van atherosclerose. Circulerende monocyten weerspiegelen de lokale situatie zoals die in de plaque is. Deze informatie kan gebruikt worden, zoals we hebben laten zien in hoofdstuk 5, voor de diagnose van hart- en vaatziekten. Toekomstig onderzoek is noodzakelijk om niet alleen biomarkers te identificeren waaraan de door ons ontdekte antilichamen binden, maar ook om de perspectieven van het gebruik van andere eigenschappen van de monocyten zoals hun activatiepotentieel als marker voor hart- en vaatziekten in kaart te brengen. 




\section{Curriculum Vitae}

\section{Jeroen J.T. Otten}

Personal Information

Date of Birth: 05 November 1983

Place of Birth: Sittard, The Netherlands

\section{Education}

\section{PhD Fellowship}

Maastricht University

Maastricht, The Netherlands

June 2009 - May 2013

Promotor: Prof. Dr. Erik A.L. Biessen

Master Forensic Science

University of Amsterdam

Amsterdam, The Netherlands

September 2006 - August 2008

\section{Bachelor Molecular Life Sciences}

Maastricht University

Maastricht, The Netherlands

September 2003 - August 2006

Atheneum (Nature \& Health, Nature \& Technology)

Graaf Huyn College

Geleen, The Netherlands

September 1996 - August 2002

\section{Experience}

\section{Account Manager}

StemCell Technologies

Regio Nederland en Vlaanderen

September 2013 - now

\section{PhD Fellow}

Maastricht University

Maastricht, The Netherlands

June 2009 - May 2013 


\section{Scientific Researcher}

Maastricht University

Maastricht, The Netherlands

February 2009 - May 2009

- 6 Active participations at (inter)national conferences

- 9 Attended courses

Grants and prizes

\section{Travel Grant}

European Society for Clinical Investigation (ESCI)

$47^{\text {th }}$ Annual Scientific Meeting

Albufeira, Portugal

17 - 20 April 2013

\section{Best Poster Presentation}

3e Cardiovasculaire Conferentie

Nederlands Lipoproteine Club Session

Noordwijkerhout, The Netherlands

14 - 15 March 2013

\section{Oral Presentations}

\section{3e Cardiovasculaire Conferentie}

Nederlandse Lipoproteine Club Session

Noordwijkerhout, The Netherlands

14 - 15 March 2013

Scandinavian Society for Atherosclerosis Research

$17^{\text {th }}$ Annual Scandinavian Atherosclerosis

Conference, Humlebaek, Denmark

13 - 16 April 2011

\section{American Heart Association (AHA)}

Scientific Session 2010, Chicago, IL, USA

13 - 17 November 2010 
European Society for Clinical Investigation

$47^{\text {th }}$ Annual Scientific Meeting

Albufeira, Portugal

17 - 20 April 2013

World Immune Regulation Meeting VI

Innate and Adaptive Immune Response and Role of

Tissues in Immune Regulation

Davos, Switzerland

18 - 21 March 2012

$1^{\text {st }}$ Cardiovasculaire Conferentie

Noordwijkerhout, The Netherlands

17 - 18 March 2011

ATVB Early Career Networking Reception

16 November 2010

Chicago, IL, USA

\section{Publications}

Otten JJ*, Medina I*, Bermudez B, Wolfs I, Rademakers T, Wijnands E, Schurgers $L$, Biessen EA. Local but not systemic $\mathrm{CD} 115^{+}$myelocyte ablation aggravates atherogenesis. Submitted.

Schutters K, Cooper D, Kusters DHM, Chatrou MLL, Otten JJ, Donners M, De Saint-Hubert M, Bauwens $M$, Krysko DV, Vandenabeele $P$, Verbruggen $A$, Biessen EA, Perretti M, Schurgers LJ, Reutelingsperger CPM. The pro-efferocytotic RGDannexin A5 inhibits de novo atherosclerotic plaque formation in apo ${ }^{-/-}$-mice. Submitted.

Otten JJ, Van den Akker N, Rousch MJ, Eijkenboom I, Jeukens F, Gagliardi M, Jukema JW, Verrips CT, Hoefer IE, Waltenberger J, El-Khattabi M, Biessen EA. Phage display identifies novel circulating and plaque monocyte markers for advanced atherosclerosis. Submitted. 
Borissoff JI, Otten JJ, Heeneman S, Leenders P, van Oerle R, Soehnlein O, Loubele ST, Hamulyák K, Hackeng TM, Daemen MJ, Degen JL, Weiler H, Esmon CT, van Ryn J, Biessen EA, Spronk HM, ten Cate $\mathrm{H}$. Genetic and pharmacological modifications of thrombin formation in apolipoprotein e-deficient mice determine atherosclerosis severity and atherothrombosis onset in a neutrophil-dependent manner. PLoS One. 2013;8(2):e55784.

Otten JJ, de Jager SC, Kavelaars A, Seijkens T, Bot I, Wijnands $E$, Beckers L, Westra MM, Bot M, Busch M, Bermudez B, van Berkel TJ, Heijnen CJ, Biessen EA. Hematopoietic G-protein-coupled receptor kinase 2 deficiency decreases atherosclerotic lesion formation in LDL receptor-knockout mice. FASEB J. 2013 Jan;27(1):265-76.

Manca M, Otten JJ, McClellan E, Van den Akker N, Gagliardi M, Jeukens F, Waltenberger J, Kuiper J, Zonneveld AJ, Hoefer IE, Jukema JW, Pasterkamp G, Biessen EA. Circulating unstable angina pectoris patient monocytes harbor no basal transcriptomic differences. In preparation.

Otten JJ*, Wijnands E*, Christ A, Rademakers T, Wolfs I, Gabriels K, Stöger L, Van Hooren L, Schalkwijk CG, Wouters K, Biessen EA. Hypercholesterolemia exposed circulating monocytes ingest lipids and induce proinflammatory pathways. Submitted. 


$A B C$

ABCA

AMI

ANOVA

APC

Apo

BMDM

BMI

BNP

$\mathrm{CCL}$

CCR

CD

CFSE

CMP

CPT

CRP

CSF-1R

CTMM

CVD

CX3CR

$\mathrm{CXCL}$

CXCR

$D A B$

DCFDA

DNA

ECG

EDTA

Egr

ELISA

EM

EMP

ER

FACS

FCS

FDR

FITC

GAPDH
ATP-binding cassette

ATP-binding cassette transporter

Acute myocardial infarction

Analysis of variance

Allophycocyanin

Apolipoprotein

Bone marrow-derived macrophage

Body mass index

B-type natriuretic peptide

CC-motif chemokine ligand

CC-motif chemokine receptor

Cluster of differentiation

Carboxyfluorescein succinimidyl ester

Common myeloid progenitor

Camptothecin

C-reactive protein

Colony stimulating factor 1 receptor

Center for translational molecular medicine

Cardiovascular disease

CX3C-motif chemokine receptor

CXC-motif chemokine ligand

CXC-motif chemokine receptor

3,3-Diaminobenzidine

$2^{\prime}, 7^{\prime}$-dichlorofluorescein diacetate

Deoxyribonucleic acid

Electro cardiogram

Ethylenediaminetetraacetic acid

Early growth response protein

Enzyme-linked immunosorbent assay

Electron microscopy

Erythrocyte/megakaryocyte progenitor

Endoplasmatic Reticulum

Fluorescence-activated cell sorting

Fetal calf serum

False discovery rate

Fluorescein isothiocyanate

Glyceraldehyde 3-phosphate dehydrogenase 


\begin{tabular}{ll} 
G-CFU & Granulocyte colony forming unit \\
G-CSF & Granulocyte colony stimulating factor \\
GDF & Growth differentiation factor \\
gDNA & Genomic deoxyribonucleic acid \\
GFP & Green fluorescent protein \\
GM-CFU & Granulocyte/macrophage colony forming unit \\
GM-CSF & Granulocyte/macrophage colony stimulating factor \\
GMP & Granulocyte/macrophage progenitor \\
GPCR & G protein-coupled receptor \\
GRK & G protein-coupled receptor kinase \\
H\&E & Haematoxylin and eosin \\
HDL & High density lipoprotein \\
hDTR & Human diphtheria toxin receptor \\
HRP & Horse radish peroxidase \\
hSTNT & High sensitive troponin T \\
i.p. & Intraperitoneal \\
i.V. & Intravenous \\
ICAM & Intercellular cell adhesion molecule \\
IFN & Interferon \\
IL & Interleukin \\
IRF & Interferon regulating factor \\
KIf & Kruppel-like factor \\
LBP & LPS-binding protein \\
LCM & L929-conditioned medium \\
LDL & Low Density Lipoprotein \\
LDLr & Low density lipoprotein receptor \\
LPS & lipopolysaccaride \\
MaFIA & Macrophage FAS induced apoptosis \\
M-CFU & Macrophage colony forming unit \\
MCP1 & Monocyte chemo-attractant protein 1 \\
M-CSF & Macrophage colony stimulating factor \\
M-CSFr & Macrophage colony stimulating factor receptor \\
MHC & Major Histocompatibility Complex \\
MMP & Matrix metallo-protease \\
MPLSM & Multi-photon laser scanning microscopy \\
MPO & Meroxidase \\
mRNA & RNA \\
\hline &
\end{tabular}




$\begin{array}{ll}\text { NF-KB } & \text { Nuclear factor kB } \\ \text { NHF } & \text { Netherlands Heart Foundation } \\ \text { NOX } & \text { NADPH oxidase } \\ \text { NPC } & \text { Niemann-Pick disease type C protein } \\ \text { oxLDL } & \text { Oxidized low density lipoprotein } \\ \text { PBL } & \text { Peripheral blood lymphocytes } \\ \text { PBMC } & \text { Peripheral blood mononuclear cell } \\ \text { PBS } & \text { Phosphate buffered saline } \\ \text { PCA } & \text { Principal component analysis } \\ \text { PE } & \text { Phycoerythrin } \\ \text { PEG } & \text { Poly(ethylene) glycol } \\ \text { PerCP } & \text { Peridinin chlorophyl protein complex } \\ \text { PFA } & \text { Paraformaldehyde } \\ \text { PMT } & \text { Photo-multiplier tube } \\ \text { RIN } & \text { RNA integrity number } \\ \text { RNA } & \text { Ribonucleic acid } \\ \text { ROS } & \text { Reactive oxygen species } \\ \text { RT } & \text { Room temperature } \\ \text { SAP } & \text { Stable angina pectoris } \\ \text { SCD4OL } & \text { Soluble CD40 ligand } \\ \text { SEM } & \text { Standard error of the mean } \\ \text { Siglec } & \text { Sialic acid-binding lg-like lectin } \\ \text { SMA } & \text { Smooth muscle cell actin } \\ \text { SMC } & \text { Smooth muscle cell } \\ \text { SPLA } & \text { Secreted phospholipases A2 } \\ \text { SSC } & \text { Side scatter } \\ \text { STEMI } & \text { ST-elevated myocardial infarction } \\ \text { TEA } & \text { Triethylamine } \\ \text { TGF } & \text { Transforming growth factor } \\ \text { TLR } & \text { Toll-like receptor } \\ \text { TNF } & \text { Tumor necrosis factor } \\ \text { TnT } & \text { Troponin T } \\ \text { TRAP } & \text { Tartrate resistant acid phosphatase antigen } \\ \text { TUNEL } & \\ \text { UAP } & \text { VCAM } \\ \text { VLA } & \end{array}$


VLDL

VSMC

WT

WTD
Very low density lipoprotein Vascular smooth muscle cell Wild type

Western type diet 



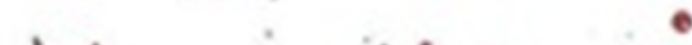

$: 1$

-

- 6

\section{Dankwoord ${ }^{\circ}$}

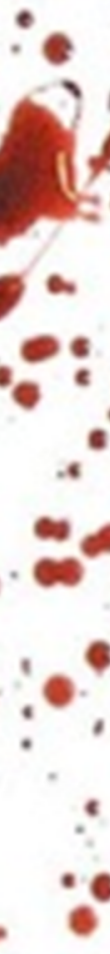
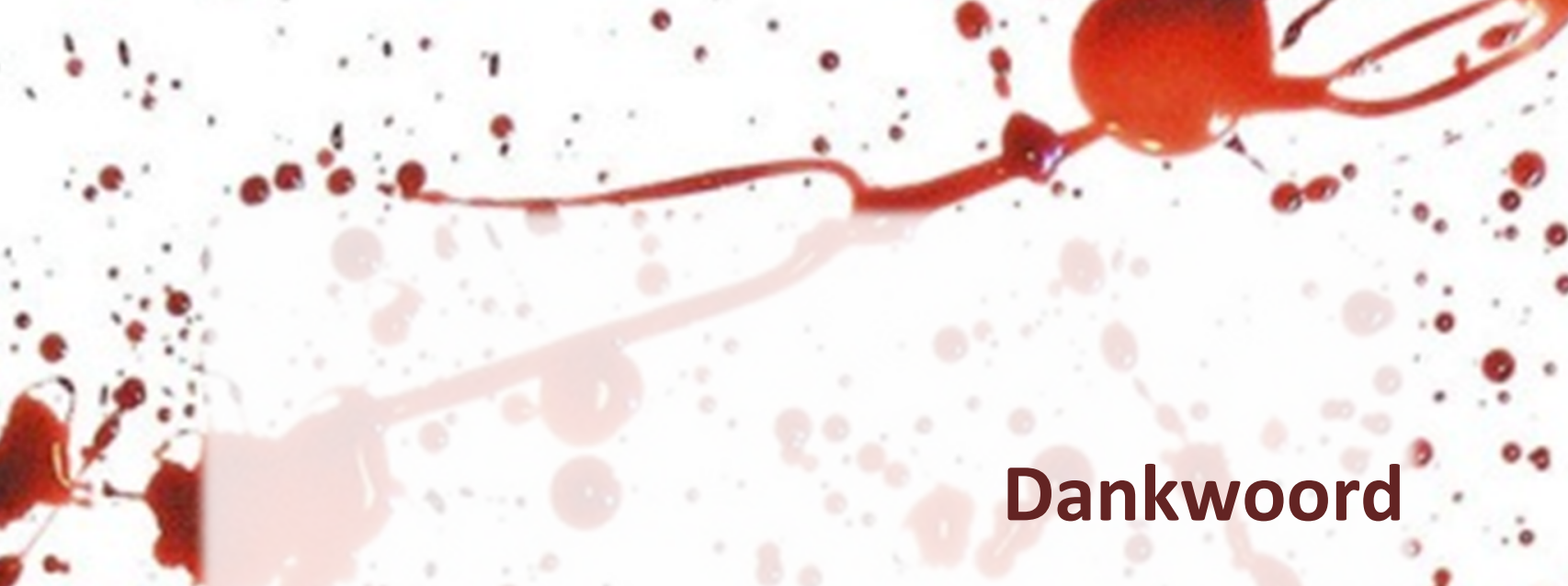

8

$0 \div$

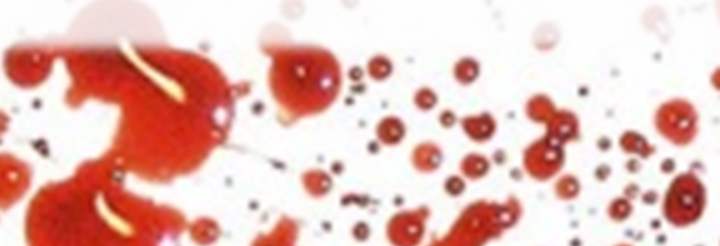

4

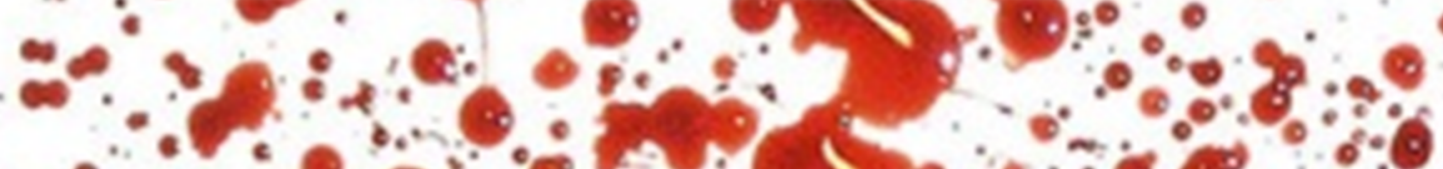

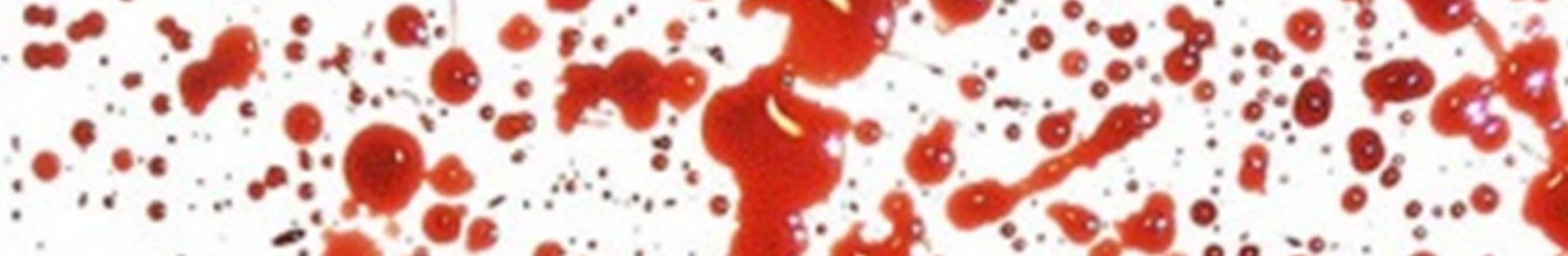
$\therefore$ *

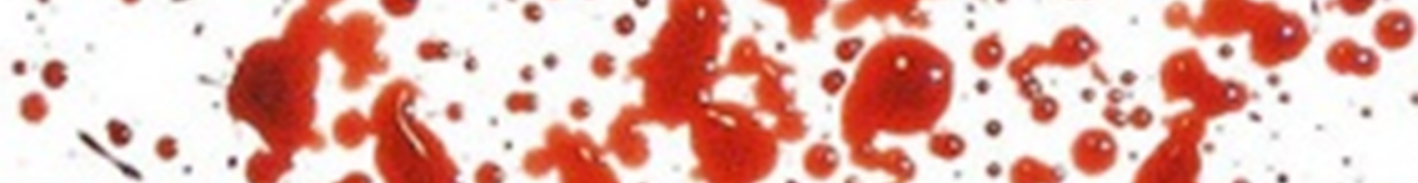

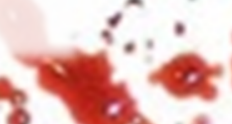

c. $\quad$ se. , "

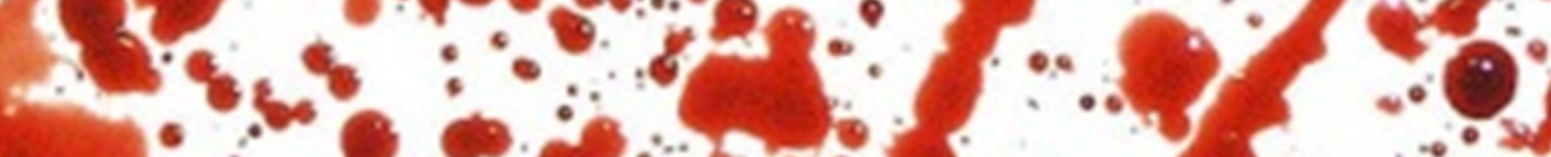
a .

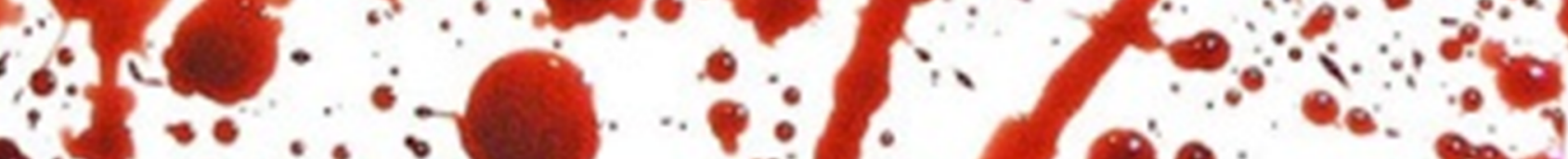

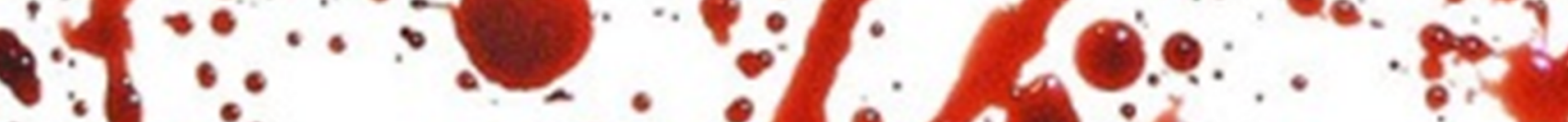
010.0 $\therefore$ $\therefore 9: \because$
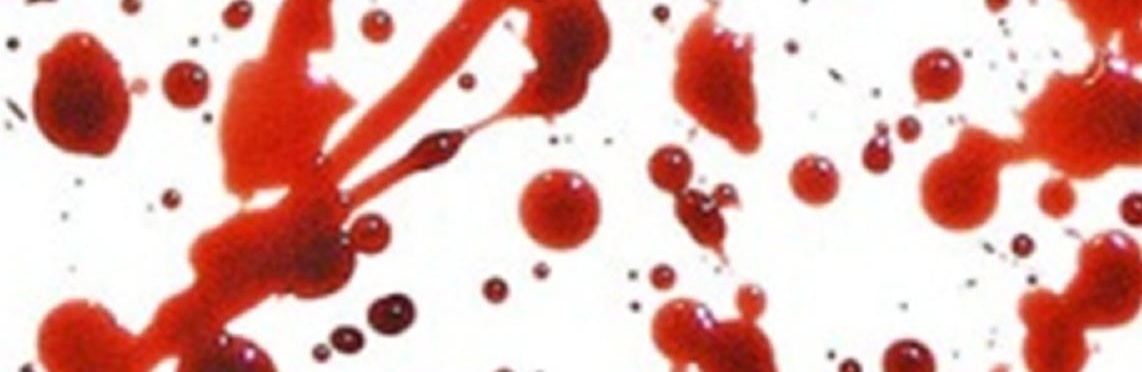
Na ruim 4 jaar werken en het schrijven van ongeveer 75.000 woorden in deze thesis, zijn dit voor veel van jullie waarschijnlijk de eerste woorden die je leest. Het is en blijft een apart idee dat iedereen het meest geïnteresseerd is in de persoonlijke verhalen aan het einde van een thesis, maar hier doe ik zelf eigenlijk ook altijd aan mee.

Het is hierbij natuurlijk van belang om te weten dat ik, net zoals iedere andere $\mathrm{AIO} / \mathrm{PhD}$ student/promovendus veel steun heb gehad van mensen binnen, maar zeker ook buiten ons werkveld. Daarom wil ik deze laatste bladzijdes van mijn boekje dan ook wijden aan het persoonlijk bedanken van iedereen die me geholpen, gesteund en gemotiveerd heeft tijdens deze indrukwekkende en leerzame periode.

Professor Dr. Biessen, beste Erik, alweer meer dan 5 jaar geleden kwamen we voor het eerst met elkaar in contact. Na een tip van Timo, solliciteerde ik binnen jouw onderzoeksgroep naar een positie als PhD student. Het werd je al snel duidelijk dat ik een andere weg had bewandeld dan menig andere kandidaat, maar gelukkig zag je toch dat ik voldoende potentie en motivatie had. Vanwege mijn forensische achtergrond zou ik eerst een proefperiode krijgen, maar al snel zagen we beiden in dat het een goede keuze was. Nu ruim 4 jaar na mijn eerste werkzaamheden binnen de EVP, is mijn thesis klaar. Ik ben je dan ook enorm dankbaar voor je vertrouwen, stortvloed aan (soms waanzinnige) ideeën en interessante gesprekken. Ik zal ook zeker nooit het schoen-incident in Chicago vergeten of ons etentje daar bij Weber grill! Daarnaast heb ik zowel mijn eerste als laatste officiële werkdag gebruik mogen maken van jouw kantoor, wat toch wel bijzonder is. Hoewel ik inmiddels de academische wereld verlaten heb, weet ik zeker dat alles wat ik hier geleerd heb me zeker verder zal helpen in de toekomst. En helemaal klaar ben je in de wetenschappelijke wereld natuurlijk nooit, aangezien we zeker nog enkele manuscripten gepubliceerd willen krijgen! Nogmaals dank voor alles in de afgelopen jaren en we zullen elkaar ongetwijfeld nog vaak genoeg tegenkomen.

Hoewel ik geen officiële co-promotor heb gehad tijdens mijn promotie, heb ik wel van één iemand in het bijzonder zeer veel steun en vriendschap mogen ontvangen, hè opper-paranimf! Beste Timo (of moet ik zeggen TimoPedia) jij bent een van de redenen geweest waardoor ik in deze helse wereld ben 
beland die men ook wel "research" noemt. En ik moet zeggen, ik heb er geen moment spijt van gehad. Aangezien we het grootste gedeelte van de afgelopen 4 jaar op dezelfde kamer hebben doorgebracht, is onze vriendschap alleen maar hechter geworden, en de 7-jaar regel hebben we duidelijk gebroken. Zoals je ook al in een van je stellingen omschreef: "I never did give anybody hell. I just told the truth and they thought it was hell." Ik weet zeker dat genoeg mensen die met ons samen een kamer hebben gedeeld, dit zeker beamen. Daarbij hebben we enkele keren samen een congres bezocht, waarbij Denemarken wel één van de mooiste congressen, lees: uitstapjes ;-) was. Helaas, zullen onze professionele werelden zich vanaf nu enigszins scheiden, maar ik weet zeker dat we in de toekomst nog vele dingen samen zullen beleven. Ik wens je veel succes met je weg naar Professor Timo, die je waarschijnlijk eerst naar het Hoge Noorden zal leiden, maar ik ben ervan overtuigd dat de toekomst je toch wel weer een keer naar het Zuiden terug zal brengen en anders weet ik je in mijn nieuwe functie ook wel weer te vinden!

Beste Stephan, we kennen elkaar al bijna 30 jaar en hoewel je niet uit het wetenschapswereldje komt, heb je het aangedurfd om mij als paranimf te ondersteunen, waar ik heel erg blij mee ben. We hebben in de lange tijd die we elkaar kennen zeer veel samen meegemaakt, in ieder geval veel te veel om zelfs maar in het kort hier neer te schrijven. Wel weet ik zeker dat ik op jou kan terugvallen, en dat is iets wat voor mij heel belangrijk is. Binnen de Ealènjers vormen we de laatste jaren ook al een mooi team binnen het bestuur, en drinken we allebei graag een pilsje. Je beland dankzij mij nu even in een wonderbaarlijke wereld, maar met ons carnavalsverleden zijn we natuurlijk heel wat gewend! Ik wens jou en Britta een hele mooie toekomst samen toe en kijk uit naar de volgende 30 jaar en alles wat die tijd ons zal brengen.

Binnen de EVP zijn er natuurlijk nog veel meer mensen die ik moet, maar vooral ook wil bedanken.

Ow FACS-koning...;-) Beste Erwin, jij was een van de personen binnen de afdeling die al wist wie ik was voordat ik ook maar was begonnen. Via alle kanalen die er zijn met het Orbis werd er veel informatie uitgewisseld, waardoor mijn komst al was aangekondigd. De afgelopen jaren hebben we extreem veel uren samen gebrainstormd over alles wat maar met FACS (of wat dan ook) te maken had. Hoewel ik denk dat ik je zeker ook wel één of twee 
dingen heb kunnen leren, heb ik vooral ongelooflijk veel van jou geleerd. Uiteindelijk zijn we zelfs nog samen op vakantie geweest met Orbis naar Oostenrijk en voor het werk naar Zwitserland, waarbij we tussendoor zelfs nog een congres hebben bezocht. Ik weet zeker dat de monocyte-studie nog tot een mooie publicatie zal leiden. Voor de toekomst hoop ik dat we nog veel skitripjes kunnen maken, waarbij je wellicht in de toekomst iets minder lang op mij hoeft te wachten... Veel geluk gewenst voor de toekomst samen met Linda!

Beste Mat, hartelijk dank voor al je hulp met het ML-1 werk. Je was de afgelopen 4 jaar voor mij iemand op wie ik altijd kon rekenen als ik iets wilde weten over faag display, maar ook PCR en celkweek behoorden tot jouw kennisgebied. We zijn ook nog een paar keer naar Dortmund geweest om treintjes te bekijken, want dat is toch een mooie hobby die we delen. Ik hoop dat je binnenkort een kamer in huis mag gaan gebruiken om ook je eigen spoor weer te kunnen opbouwen. Ik reserveer in ieder geval voor komend jaar het dagje Duitsland weer in mijn agenda!

Beste Karen, we hebben ongeveer even lang op de afdeling rondgelopen en zijn nu ook bijna tegelijk klaar met onze thesis. Hoewel onze onderzoeken wat verder uit elkaar lagen, hebben we heel vaak samen bij de muizen of in het lab gewerkt. Ik hoop dat de toekomst jou vol geluk toelacht, maar ik weet zeker dat jij goed terecht komt, zowel privé als op het werk.

Lieve Lieve (sorry maar blijft leuk), ondanks dat je later dan een groot gedeelte van de andere mensen binnen de groep bent gekomen, denk ik dat je een van de personen bent die niet alleen voor top onderzoek zorgt, maar ook voor een hele hoop gezelligheid en plezier. Ik heb ook erg genoten van alle keren dat je samen met Lucas naar de borrels kwam, jong geleerd is oud gedaan, hè! Voor mij was verder het schminken in Aachen wel een hoogtepunt. Heel veel respect dat je me liet kliederen! Veel geluk voor de toekomst samen met Pieter, Lucas en Marieke.

Lieber Anette, wir haben 4 Jahren zusammen gearbeitet und ich habe immer sehr viel Bewunderung gehabt für deine Engagement. Sie waren schnell bei der Planung und Durchführung von Experimenten, aber immer mit der höchste Qualität. Ich glaube das wir noch viel von dich hören würde, und das du sicher 
im einer der top Labos der Welt arbeiten solltest. Ich hoffe das wir uns noch öfter sehen und vielleicht könnten wir nochmals Schifahren machen, oder am wenigsten ein Bier (und Jägermeister) trinken. Viel Glück und bis Bald!

Beste Ine, sinds je naar de patho gekomen bent, heb je een zware tijd gehad, geloof ik. Je moest immers een kamer delen met onder andere Timo en mij, en wij zijn nu eenmaal nooit rustig hè. Wel heb je met succes deelgenomen aan onze cursus, waarvoor nogmaals mijn complimenten. Ik wil je ook danken voor alle hulp die je hebt geboden, met het uitvoeren van allerlei experimenten. Zelfs al was je dag drie keer volgepland, dan nog vond je altijd ruimte om een ander en dus ook mij te helpen. Ik hoop dat je thesis snel volgt en geloof me maar als ik zeg dat dit allemaal goed gaat komen. Veel succes met je verdere carrière en ik kom graag nog eens BBQ-en.

Dear David, I still do not know which language I should choose when we are talking. However, most often we just stuck to English. We have spent a lot of time together in the lab but also in our room. And I have to say that it became quiet after you left, no more German swearing or loud music coming from your ears. Ich möchte Ihnen für die letzten Jahre bedanken und wünschen Ihnen viel Glück bei Ihrer nächsten Herausforderung in der Forschung. En voor een biertje ben ik altijd wel te bereiken!

Judith, allereerst wil ik je danken voor de uitdaging die je voor me hebt neergelegd, om een langer dankwoord te schrijven dan Timo (hoezo Mission Impossible?). Ik doe in ieder geval mijn best om in de buurt te komen ;-) We hebben sinds je (terug)komst naar de pathologie veel samengewerkt, waarbij ik ook veel van je heb geleerd over wetenschap. Je bent nu ongeveer één jaar moeder van Sophie en ik heb het gevoel dat het je zeer goed bevalt, ondanks de vaak gebroken nachten. Maar ik weet dat een kleintje uiteindelijk toch het belangrijkste is, en ook hetgene waarvoor je aan het einde van de dag graag naar huis gaat. Succes met je toekomst en ik weet zeker dat we over een paar jaar naar jou inauguratie mogen komen. Veel plezier met zowel je professionele en persoonlijke leven en de toekomstige uitbreiding van je gezinnetje. 
Onze kamer heeft de laatste jaren ook een behoorlijke buitenlandse invloed gehad. Naast de Belgische enclave, was er ook een duidelijk Duitse invloed.

Thomas, je was de eerste AIO bij Judith en bent onze borrel-specialist. Zodra er iets te vieren was zorgde jij ervoor dat dit een geslaagde avond was, waarbij er bij voorkeur natuurlijk duits bier aanwezig was. Veel succes met je onderzoek hier en in Finland.

Elke jij was de tweede Duitse invloed in onze kamer en ook jij werkte voor Judith. Je weet altijd heel goed wat je wilt en duikt er dan bovenop. Jouw inzet en motivatie zijn voor mij een echt voorbeeld. Veel succes met nog alles wat de komende jaren brengen.

Floor, jij bent de afgelopen tijd in de kamer mijn buurvrouw geweest. Hoewel je veel bezig was met patientendata, statistiek en allemaal van die dingen, heb ik bewondering voor je doorzettingsvermogen. Ik denk dat ik allang gillend was weggerend. Veel succes in Amsterdam met je opleiding.

Beste Sylvia, je bent de afgelopen jaren degene geweest waar ik met allerlei vragen terecht kon, maar vooral op het gebied van vragen omtrent de DEC en classificatie van plaques kon ik altijd op jou rekenen. Dank voor al je hulp en wijsheden.

Bart, bedankt voor al je gezellige en vooral vaak ook verrassende gesprekken die we hebben gehad. Hoewel we vaak dachten dat je een introverte Vlaming was, kwamen er vaak heel onverwachte dingen uit je mond. Je positie is niet altijd even makkelijk, maar ik weet zeker dat je er voor gaat zorgen dat je promotie een groot succes gaat worden.

Hoewel immuno's en quantificeren nooit mijn favoriete bezigheid zijn geweest was dit toch een belangrijk onderdeel van mijn onderzoek. Jack ik wil je dan ook heel erg bedanken voor het schrijven en aanpassen van alle quips zodat deze 'lijdensweg' iets draaglijker werd ;-).

Beste Petra, hoewel je officiële status in onze groep net iets anders was, ben je absoluut belangrijk geweest voor de EVP, maar ook voor mij. Als ik weer eens een onmogelijke bestelling had, wist ik dat je er alles aan zou doen om te regelen dat dit in orde kwam. Daarnaast heb ik ook erg genoten van de 
uitstapjes, carnaval, maar ook onze wandelingen naar het station als we allebei weer eens naar de trein moesten. Veel succes met je nieuwe baan en dank voor je hulp en gezelligheid.

Beste Anique, je hebt me leren snijden en immuno's leren uitvoeren, en hoewel het nog altijd geen hobby is, zie ik er wel de meerwaarde van in. Hartelijk dank voor al je hulp en de gezellige gesprekken.

Clairy, geregeld stond ik weer eens naast je voor een speciale kleuring, zoals een Movat. Dank voor deze mooie kleuringen die zeker hebben bijgedragen aan alle kleur in mijn thesis.

Danielle, Ingrid, Audrey, ook jullie hulp is voor mij zeer belangrijk geweest de afgelopen jaren. Danielle, als er ook maar iets geregeld moest worden of het nu een vlucht naar een congres was of een afspraak met Erik, jij zorgde dat het snel en efficiënt geregeld werd. Hoewel er soms enige chaos was met agenda's en afspraken die Erik verschoof, kwam dankzij jou alles uiteindelijk toch weer in orde.

Marco, I have to admit that, despite numerous meetings on your research (Bioinformatics), most of it is still not completely clear to me. Nevertheless, I can see the additive value of your analyses in many projects and I would like to thank you for your help and also in sharing our frustration we often experienced with some projects ;-)

Beste, Marjo, Marion, Emiel, hoewel jullie nog altijd bij MolGen jullie plekje hebben zijn jullie ook zeker onderdeel van ons EVP-clubje. Marjo dank voor je hulp met onder andere de LysM dieren. Emiel, voor mij ben jij degene die mijn macrofaag vragen kon beantwoorden, dank hiervoor. Beste Marion, zowel voor mijn studies maar ook als er iets was met muizen, ik kon altijd met mijn vragen bij jou terecht. Heel erg bedankt hiervoor het heeft me veel geleerd over de pathologie.

Dear Anjana, thank you for your input also when I was applying for a new job and all the best in research. 
Jasper, we hebben kort nog even een kamer gedeeld en hoewel je 3FM nog moet leren waarderen weet ik zeker dat jou een mooie toekomst wacht.

Chiara, Pawel, and Sebastièn, I think you are a great asset to our department, but unfortunately we did not really have the time to work together. Good luck with your projects!

Ook heb ik gedurende de afgelopen vier jaar een aantal studenten mogen begeleiden tijdens hun stages. Matthias, Marcel, Ivo, Luuk en Jos, bedankt voor jullie harde werk, maar ook gezelligheid tijdens de stages binnen diverse projecten en veel succes gewenst met jullie wetenschappelijke carrières.

Daarnaast zijn er ook veel mensen geweest die onze afdeling inmiddels hebben verlaten, maar die wel een heel belangrijke bijdrage hebben geleverd aan deze thesis op welke manier dan ook.

Prof. Dr. Lutgens, Beste Esther, vanaf het eerste gesprek dat ik met je voerde tijdens mijn sollicitatie was ik onder de indruk van hetgeen je bereikt hebt. Ik wil je bedanken voor alle hulp, tips, en (wetenschappelijke) gesprekken.

Beste Linda, jouw vertrek naar Amsterdam was een groot verlies voor onze groep, niet alleen qua wetenschap, maar zeker ook voor alle gezelligheid die jij met je meebracht. Als muizenmeisje heb ik heel veel van je geleerd over muisstudies. Maar je hebt me ook geleerd hoe je moet doorgegeven als er iets besteld moet worden ;-) Veel geluk gewenst samen met Lauran en Ella.

Beste Tom, als toekomstige Dr. Dr. (en alle andere titels die nog toegevoegd gaan worden), heb ik altijd bewondering gehad voor je eindeloze inzet en vooral de onmogelijke uren die jij hebt gewerkt. Dank voor al je hulp bij injecties en stamcellen.

Verder wil ik Professor Mat Daemen, Indira, Bea, Veronica, Wouter, Mathijs G., Matthijs van G., Susanne, Brenda, Cora, Isabelle en Nadine bedanken voor jullie hulp en gezellige momenten gedurende de afgelopen jaren. 
Uiteraard zijn er binnen de afdeling Pathologie nog veel meer mensen die ik wil bedanken voor de afgelopen jaren. Allereerst natuurlijk Professor Dr. Zur Hausen. Beste Axel, hartelijk dank voor uw interesse in ons cardiovasculaire onderzoek en voor alle mogelijkheden die u mij heeft geboden.

Cor en Elly, hoewel het merendeel van jullie werk niet direct zichtbaar is, weet ik zeker dat zonder jullie de afdeling nooit zo goed zou kunnen functioneren. Dank voor jullie hulp bij de meest diverse vraagstukken.

Daarnaast wil ik Manon, Veerle, Hanneke, Muriel, Edith, Peter, Kim W, Kim S, Kim van S, Ilse en Jürgen van de 'Epi's' bedanken voor hun gezelligheid en interessante discussies tijdens de diverse meetings en wetenschapsdagen. Joep en Karen, jullie hebben veel tijd doorgebracht onder de EVP-ers toen we nog samen in de kleine demo zaten, maar ook tijdens de borrels en carnaval waren jullie er altijd bij. Ik wil jullie dan ook bedanken voor alle gesprekken over de meest vreemde dingen, van koffie tot blaasmuziek, en hoop ook snel op jullie promotie aanwezig te kunnen zijn.

Also Anke, Dorit, Ruth en Shredar thank you all. And I wish you all the best with your research in the future.

Buiten de research is er ook nog 'de kliniek'. Ook alle mensen hier wil ik danken, in het bijzonder wil ik Jolanda, Guido, Kathleen, Benoit en Carine danken voor hun bijdrage en hulp, of gewoon gezellige gesprekken.

Niet alleen binnen onze afdeling maar ook daarbuiten zijn er veel mensen die me hebben gesteund in de afgelopen jaren. Dear Julian, I have always admired your great ideas and the way you perform science, but also just the talks and emails. All the best for the future, together with your family.

Verder wil ik vanuit de afdeling Biochemie ook iedereen bedanken met wie ik de afgelopen jaren heb samengewerkt.

Beste Leon, we hebben elkaar ontmoet tijdens de proefdiercursus en hierna hebben we nog vaak interessante gesprekken gevoerd en zelfs samengewerkt voor studies van zowel Biochemie als Pathologie. Heel erg 
bedankt voor alle tips en handigheidjes, maar ook je bijdrage aan dit proefschrift.

Verder wil ik iedereen van de groepen Interne Geneeskunde en Immunologie bedanken voor alle bijdrages.

Beste Kristiaan, we hebben vooral in de laatste maanden veel samengewerkt. Ik wil je danken voor je directe bijdrages aan verschillende hoofdstukken van deze thesis en wens je veel succes met je ongetwijfeld succesvolle wetenschappelijke carrière.

Beste Jozien, ik wil je heel erg bedanken voor alle hulp die je hebt geboden bij het FACSen en sorten.

Vanuit de humane monocyten heb ik ook veel samengewerkt met Cardiologie. Ik wil dan ook Nynke, Fabienne, Mick en Daniel heel erg bedanken voor jullie hulp bij de diverse projecten.

Uiteraard zijn ook de medewerkers van het CPV heel belangrijk geweest om op een goede manier onderzoek te kunnen uitvoeren. Ik wil alle medewerkers van het CPV dan ook bedanken voor al hun hulp en ondersteuning. In het bijzonder wil ik Clarice, Rick en Harry bedanken voor jullie hulp met de muizen, Richard voor je hulp als er weer eens iets geregeld moest worden (vaak ook nog op korte termijn), en jij er altijd voor zorgde dat alles weer in orde gemaakt werd. Tot slot wil ik ook Saskia, Paulien, Marianne, Nadine en Angeli nog bedanken voor jullie hulp op verschillende gebieden.

Mijn promotie onderzoek was onderdeel van het CTMM project Circulating Cells en ik wil dan ook alle collega's binnen onze werkgroep hartelijk danken. Matthias, ondanks dat je aan de andere kant stond en meer keek naar technische en mechanische eigenschappen van cellen, werkte we wel met dezelfde cellen. Ellen, vaak hebben we ons afgevraagd wat wij als monocytemensen nu moesten binnen het project, maar ik denk dat we allebei mooie resultaten hebben verkregen en ook een goede bijdrage hebben kunnen leveren. Daarnaast wil ik Menno, Arthur, Imo, Mark, Joost, Loes, Thijs, Bert, Peggy, en alle andere mensen die binnen CTMM hebben gewerkt hartelijk danken! 
Beste Dr. Sikkink en Dr. Van den Akker, ook jullie wil ik hartelijk danken voor de samenwerking bij het verzamelen van plaque materiaal, alsook alles wat ik heb mogen leren van alles op de OK in Sittard. Hopelijk levert de samenwerking in de toekomst nog vele mooie resultaten en publicaties op.

Daarnaast heb ik natuurlijk de afgelopen jaren nog met vele andere mensen mogen samenwerken in binnen- en buitenland.

Professor Halvorsen, dear Bente and dear Tuva thank you for the opportunity to work shortly in your lab to assist in some flow cytometry experiments. For me this was an extremely interesting and educative experience. Good luck with your future research and I am always willing to come back for some more assistance!

Verder wil ik ook alle mensen met wie ik binnen Nederland heb samengewerkt hartelijk bedanken. Beste Saskia, Ilze, Annemieke en Cobi, heel erg bedankt voor alle hulp en input voor het GRK2 stuk. Dit was mijn eerste grote studie en mede dankzij jullie is dit een mooie publicatie geworden.

Ook wil ik graag nog een aantal (oud-)studiegenoten bedanken voor al hun hulp die ik tijdens mijn studie, maar ook tijdens mijn promotie heb gehad. En natuurlijk alle daarbij behorende gezellige middagen en avonden, in het bijzonder Dennis, Ivo, Rianne, Ryanne en Siamack bedanken voor alles.

Beste Jennifer, inmiddels ben je samen met Daniel op avontuur gegaan in Canada. Hoewel dit betekent dat we de komende tijd niet zoveel gezellige etentjes zullen hebben, weet ik zeker dat in het onderzoek een hele mooie toekomst op je ligt te wachten. Het voordeel is wel dat ik de komende tijd nog kan blijven oefenen om in de toekomst ook eens echt mee te kunnen doen als we weer eens het MLW spel 'SET' spelen. Ik wens jou en Daniel heel veel geluk en succes toe zowel in jullie werk als daarbuiten.

Beste Roger, we hebben vanaf het eerste begin toen we begonnen aan de bachelor MLW altijd veel plezier gehad en menig uurtje doorgebracht met troeven. Je hebt inmiddels de academische wereld verlaten en bent samen met je gezinnetje naar het 'hoge noorden' vertrokken om daar aan de slag te gaan. Gelukkig ben je nog vaak in Limburg te vinden en weten wij ook de weg 
naar Nootdorp inmiddels te vinden. Veel geluk gewenst samen met Nathalie en Mads en wellicht brengt de toekomst je wel weer terug naar het Limburgse Land.

Uiteindelijk is het toch nog een flinke dankwoord geworden en hoop ik iedereen te hebben bedankt, door de lange lijst van namen sluit ik niet uit dat ik toch nog iemand ben vergeten te bedanken. Daarom wil ik nogmaals iedereen hartelijk danken voor alle hulp, input, gesprekken, gezelligheid en alles meer gedurende de afgelopen jaren. Hierbij wil ik ook al mijn ooms, tantes, neven en nichten, maar ook iedereen binnen de verenigingen waarin ik actief ben (geweest), bedanken voor alles en hopelijk begrijpen jullie nu een beetje wat ik tijdens mijn promotie nu echt heb gedaan.

Buiten het werk heb ik ook veel steun mogen ontvangen van mijn schoonfamilie, Erna, Ben en Ludia, Pascal en Chantal, en Jessica en Rob allemaal heel erg bedankt voor jullie steun, interesse en lekkere etentjes.

Beste Oma Lenie, ik wil u hierbij ook hartelijk danken voor al uw steun, maar ook interesse in alles waar ik de afgelopen jaren mee bezig ben geweest. Ik hoop dat we de komende jaren nog veel leuke en mooie momenten moge beleven met de hele familie.

Beste Michel en Rianne, ook jullie wil ik bedanken voor jullie ondersteuning. De afgelopen jaren is er veel veranderd en jullie hebben nu ook jullie eigen plekje gevonden in Awt-Gelaen bij pap en mam om de hoek. Ik hoop dat we de komende jaren nog vaak samen kunnen genieten van al het goede dat op ons afkomt. Veel succes en geluk toegewenst!

Beste Thomas en Mandy, uiteraard wil ik ook jullie bedanken. Momenteel zijn jullie hard aan het zoeken naar een mooi plekje hier in het zuiden van het land, om zo al het gereis tussen Limburg en Holland wat te kunnen beperken. Ik weer zeker dat jullie hier ook snel in zullen slagen! Dank voor het plezier dat jullie me de afgelopen jaren geboden hebben. Ook voor jullie veel succes en geluk toegewenst. 
Lieve Pap en Mam, de afgelopen 30 jaar hebben jullie altijd voor mij klaar gestaan. Tijdens alle hoogte- maar ook dieptepunten wist ik dat jullie er voor mij waren om vreugde en verdriet te delen. Alles wat ik, maar ook Michel en Thomas bereikt hebben, is voor een groot gedeelte aan jullie te danken. Hoewel woorden waarschijnlijk niet kunnen dekken hoeveel jullie voor mij betekend hebben, wil ik jullie heel erg bedanken voor alles wat jullie voor mij hebben gedaan en de wijsheid, vertrouwen en liefde die jullie mij hebben gegeven.

Tot slot natuurlijk wil ik natuurlijk mijn lieve vrouw Stefanie en zoon Finn bedanken voor de liefde en het geluk die jullie mij bieden. Lieve schat, dankzij al jouw steun en hulp heb ik de afgelopen jaren kunnen werken aan dit boekje. Hoewel ik vaak lange dagen maakte of af en toe weg was op congres, zorgde jij ervoor dat alles goed verliep. Afgelopen jaren is er veel veranderd, we zijn getrouwd en onze zoon Finn is geboren. Wat de toekomst nog zal brengen weet niemand, maar dat we dit samen gaan beleven weet ik zeker. Ik hou heel veel van jullie allebei.

Jeroen

Als je de richting van de wind niet kunt veranderen, verander dan de stand van je zeilen 